\title{
Nickel-Catalyzed Borylation of Benzylic Ammonium Salts: Stereospecific Synthesis of Enantioenriched Benzylic Boronates
}

\author{
Corey H. Basch, Kelsey M. Cobb, Mary P. Watson* \\ Department of Chemistry and Biochemistry, University of Delaware, Newark, DE 19716 \\ mpwatson@udel.edu
}

\section{SUPPORTING INFORMATION}

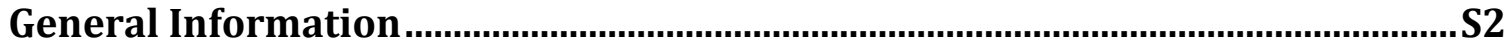

Stereospecific Borylation of Benzylic Ammonium Salts .........................................

General Procedure A: Borylation of Naphthyl-Substituted Benzylic Ammonium

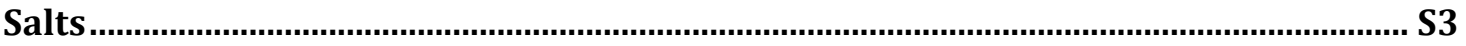

General Procedure B: Borylation of Non-Naphthyl-Substituted Benzylic Ammonium

Salts

General Procedure C: Oxidation of Benzylic Boronates to Benzylic Alcohols for

Determination of Enantiomeric Excess (ee).

Gram-Scale Synthesis of (S)-2-(1-(6-methoxynaphthalen-2-yl)ethyl)-4,4,5,5-

tetramethyl-1,3,2-dioxaborolane (2c) ..........................................................S24

Preparation of Benzyl Ammonium Salts.............................................................. S24

General Procedure D: Preparation of $(S)-N, N, N$-trimethyl-1-(naphthalen-1-

yl)ethanaminium trifluoromethanesulfonate (1b)

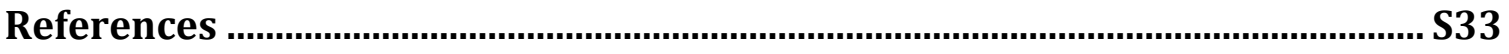

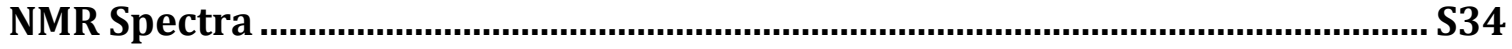

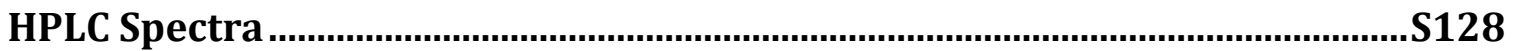




\section{General Information}

Reactions were performed in oven-dried vials with Teflon-lined caps or in oven-dried round-bottomed flasks unless otherwise noted. Flasks were fitted with rubber septa, and reactions were conducted under a positive pressure of $\mathrm{N}_{2}$. Stainless steel syringes or cannulae were used to transfer air- and moisture-sensitive liquids. Flash chromatography was performed on silica gel $60(40-63 \mu \mathrm{m}, 60 \AA)$ unless otherwise noted. Commercial reagents were purchased from Sigma Aldrich, Acros, Fisher, Strem, TCI, Combi Blocks, Alfa Aesar, or Cambridge Isotopes Laboratories and used as received with the following exceptions: Bis(pinacolato)diboron, bis(neopentyl glycolato)diboron, and bis(hexylene glycolato)diboron were purchased from Sigma Aldrich and immediately placed in a $\mathrm{N}_{2}{ }^{-}$ atmosphere glovebox for storage. $\mathrm{Ni}(\mathrm{OAc})_{2} \cdot 4 \mathrm{H}_{2} \mathrm{O}$ was purchased from Alfa Aesar and donated by Astra Zeneca. Methyl trifluoromethanesulfonate (MeOTf) was purchased from TCI and used directly. 1,3-Bis(cyclohexyl)imidazolium tetrafluoroborate $\left(\mathrm{ICy} \cdot \mathrm{HBF}_{4}\right)$ was purchased from Sigma Aldrich and used as received.. THF was dried by passing through drying columns, then degassed by sparging with $\mathrm{N}_{2}$ and stored over activated $4 \AA \mathrm{MS}$ in a $\mathrm{N}_{2}$-atmosphere glovebox. ${ }^{1}$ Commercially available enantioenriched amines were purchased from Alfa Aesar or Sigma Aldrich and used as received. Enantioenriched amines that were not commercially available were obtained through Grignard or hydride additions of Ellman's sulfinimines. ${ }^{2}$ Dimethyl benzyl amines were prepared using Escheweiler-Clarke conditions or reductive amination of the corresponding primary benzyl amine with formaldehyde. ${ }^{3}$ In some instances oven-dried potassium carbonate was added into $\mathrm{CDCl}_{3}$ to remove trace amount of acid. Proton nuclear magnetic resonance $\left({ }^{1} \mathrm{H}\right.$ NMR) spectra, carbon nuclear magnetic resonance $\left({ }^{13} \mathrm{C}\right.$ NMR) spectra, fluorine nuclear magnetic resonance spectra $\left({ }^{19} \mathrm{~F}\right.$ NMR), and silicon nuclear magnetic resonance spectra $\left({ }^{29} \mathrm{Si} \mathrm{NMR}\right)$ were recorded on both $400 \mathrm{MHz}$ and 600 $\mathrm{MHz}$ spectrometers. Boron nuclear magnetic resonance spectra $\left({ }^{11} \mathrm{~B} \mathrm{NMR}\right)$ were recorded on a $600 \mathrm{MHz}$ spectrometer. Chemical shifts for protons are reported in parts per million downfield from tetramethylsilane and are referenced to residual protium in the NMR solvent $\left(\mathrm{CHCl}_{3}=\delta\right.$ 7.26). Chemical shifts for carbon are reported in parts per million downfield from tetramethylsilane and are referenced to the carbon resonances of the solvent $\left(\mathrm{CDCl}_{3}=\delta 77.2\right)$. Data are represented as follows: chemical shift, multiplicity 
( $\mathrm{br}=$ broad, $\mathrm{s}=$ singlet, $\mathrm{d}=$ doublet, $\mathrm{t}=$ triplet, $\mathrm{q}=$ quartet, $\mathrm{p}=$ pentet, $\mathrm{m}=$ multiplet, $\mathrm{dd}$ $=$ doublet of doublets, $\mathrm{h}=$ heptet$)$, coupling constants in Hertz $(\mathrm{Hz})$, integration. Infrared (IR) spectra were obtained using FTIR spectrophotometers with material loaded onto a $\mathrm{KBr}$ plate. The mass spectral data were obtained at the University of Delaware mass spectrometry facility. Optical rotations were measured using a $2.5 \mathrm{~mL}$ cell with a $0.1 \mathrm{dm}$ path length. Melting points were taken on a Stuart SMP10 instrument.

\section{Stereospecific Borylation of Benzylic Ammonium Salts}

\section{General Procedure A: Borylation of Naphthyl-Substituted Benzylic Ammonium Salts}

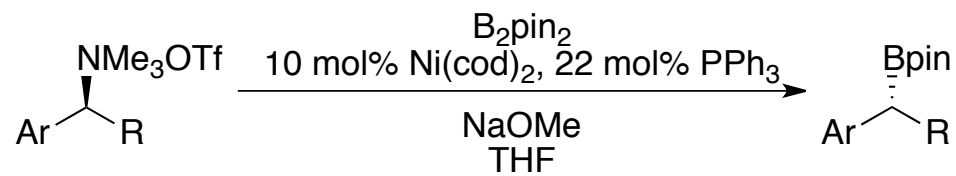

In a $\mathrm{N}_{2}$-atmosphere glovebox, $\mathrm{Ni}(\mathrm{cod})_{2}(8.3 \mathrm{mg}, 0.030 \mathrm{mmol}, 10 \mathrm{~mol} \%), \mathrm{PPh}_{3}(4.4$ mg, $0.066 \mathrm{mmol}, 22 \mathrm{~mol} \%$ ), NaOMe (24 mg, $0.45 \mathrm{mmol}, 1.5$ equiv), $\mathrm{B}_{2} \mathrm{pin}_{2}$ (114 mg, $0.45 \mathrm{mmol}, 1.5$ equiv), and ammonium salt 1 ( $0.30 \mathrm{mmol}, 1.0$ equiv) were weighed into a 1-dram vial equipped with a magnetic stir bar. THF $(1.5 \mathrm{~mL}, 0.2 \mathrm{M})$ was added, and the vial was capped with a Teflon-lined cap and removed from the glovebox. The mixture was stirred at room temperature for $24 \mathrm{~h}$. The reaction mixture was then diluted with $\mathrm{Et}_{2} \mathrm{O}(2.5 \mathrm{~mL})$ and quickly filtered through a short plug of Celite ${ }^{\circledR}$, which was then rinsed with $\mathrm{Et}_{2} \mathrm{O}(\sim 10 \mathrm{~mL})$. The filtrate was concentrated and purified by silica gel chromatography to give the benzylic boronate product. The benzylic boronate was then converted to the corresponding benzylic alcohol via oxidation (see General Procedure C below) to determine the enantiomeric excess (ee). 


\section{General Procedure B: Borylation of Non-Naphthyl-Substituted Benzylic Ammonium Salts}

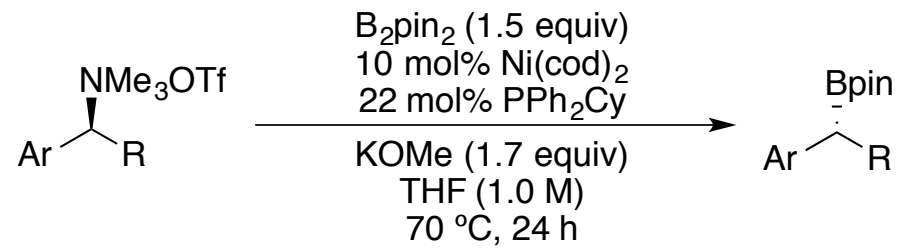

In a $\mathrm{N}_{2}$-atmosphere glovebox, $\mathrm{Ni}(\operatorname{cod})_{2}(8.3 \mathrm{mg}, 0.030 \mathrm{mmol}, 10 \mathrm{~mol} \%), \mathrm{PPh}_{2} \mathrm{Cy}(18$ $\mathrm{mg}, 0.066 \mathrm{mmol}, 22 \mathrm{~mol} \%$ ), KOMe (38 mg, $0.45 \mathrm{mmol}, 1.7$ equiv), $\mathrm{B}_{2} \mathrm{pin}_{2}$ (114 mg, $0.45 \mathrm{mmol}, 1.5$ equiv), and ammonium salt 1 ( $0.30 \mathrm{mmol}, 1.0$ equiv) were weighed into a 1-dram vial equipped with a magnetic stir bar. THF $(0.3 \mathrm{~mL}, 1.0 \mathrm{M})$ was added and the vial was capped with a Teflon-lined cap and removed from the glovebox. The mixture was stirred at $70{ }^{\circ} \mathrm{C}$ for $24 \mathrm{~h}$. The reaction mixture was then diluted with $\mathrm{Et}_{2} \mathrm{O}(2.5 \mathrm{~mL})$ and quickly filtered through a plug of Celite ${ }^{\circledR}$, which was then rinsed with $\mathrm{Et}_{2} \mathrm{O}(\sim 10$ $\mathrm{mL}$ ). The filtrate was concentrated and then purified by silica gel chromatography to give the benzylic boronate product. The benzylic boronate was then converted to the corresponding benzylic alcohol via oxidation (see General Procedure C below) to determine the enantiomeric excess (ee).

\section{General Procedure C: Oxidation of Benzylic Boronates to Benzylic Alcohols for Determination of Enantiomeric Excess (ee).}

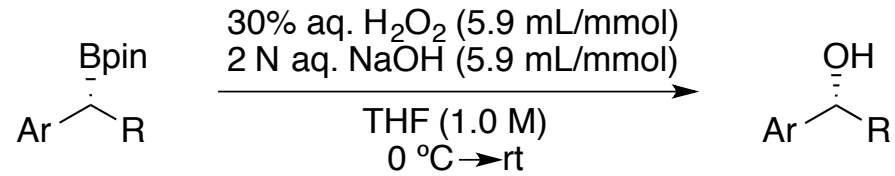

A solution of the benzylic boronate 2 (1.0 equiv) and $\mathrm{Et}_{2} \mathrm{O}(0.017 \mathrm{M})$ was cooled to 0 ${ }^{\circ} \mathrm{C}$. Aqueous $\mathrm{NaOH}(2 \mathrm{~N}, 5.9 \mathrm{~mL} / \mathrm{mmol}$ of 2$)$ was added, followed by aq. $\mathrm{H}_{2} \mathrm{O}_{2}(30 \%, 5.9$ $\mathrm{mL} / \mathrm{mmol}$ of 2). The mixture was stirred and allowed to warm slowly to room temperature overnight. The reaction mixture was diluted with $\mathrm{H}_{2} \mathrm{O}$ and $\mathrm{Et}_{2} \mathrm{O}$, and the layers were separated. The aqueous layer was extracted with $\mathrm{Et}_{2} \mathrm{O}(3 \times 20 \mathrm{~mL})$. The combined organic layers were dried $\left(\mathrm{MgSO}_{4}\right)$, filtered, and concentrated. The crude mixture was purified via silica gel chromatography to afford benzylic alcohol $\mathbf{3}$ for ee 
determination. For duplicate experiments, alcohol $\mathbf{3}$ was isolated once via column chromatography (to verify high yield in the oxidation) and once via preparatory thin-layer chromatography under the same mobile-phase conditions.

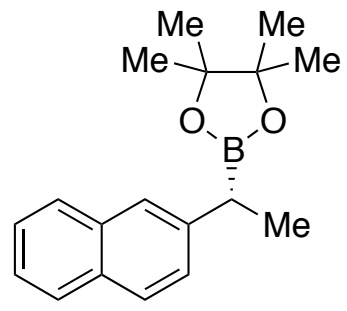

(R)-4,4,5,5-tetramethyl-2-(1-(naphthalen-2-yl)ethyl)-1,3,2-dioxaborolane (2a). Prepared via General Procedure A using ammonium salt 1a (amine purchased in $>99 \%$ ee). The crude mixture was purified by silica gel chromatography (5\% EtOAc/hexanes) to give 2a (run 1: $69 \mathrm{mg}, 82 \%$; run 2: 79\%) as a white solid: ${ }^{1} \mathrm{H} \mathrm{NMR}\left(400 \mathrm{MHz}, \mathrm{CDCl}_{3}\right.$ ) $\delta 7.83-7.75(\mathrm{~m}, 3 \mathrm{H}), 7.67(\mathrm{~s}, 1 \mathrm{H}), 7.48-7.37(\mathrm{~m}, 3 \mathrm{H}), 2.64(\mathrm{q}, J=7.4 \mathrm{~Hz}, 1 \mathrm{H}), 1.46(\mathrm{~d}$, $J=7.6 \mathrm{~Hz}, 3 \mathrm{H}), 1.23(\mathrm{~s}, 6 \mathrm{H}), 1.22(\mathrm{~s}, 6 \mathrm{H}) ;{ }^{13} \mathrm{C} \mathrm{NMR}\left(101 \mathrm{MHz}, \mathrm{CDCl}_{3}\right) \delta 142.7,134.0$, $131.8,127.8,127.7,127.6,127.4,125.8,125.4,124.9,83.5,24.8,24.8,17.0 .{ }^{4}$ The spectral data match that previously reported in the literature. ${ }^{5}$

Boronate 2a was oxidized to alcohol 3a via General Procedure C. The enantiomeric excess was determined to be $99 \%$ (run 1: $99 \%$ ee; run 2: $99 \%$ ee) by chiral HPLC analysis. See alcohol 3a below.<smiles>CC(O)c1ccc2ccccc2c1</smiles>

(R)-1-(naphthalen-2-yl)ethanol (3a). Prepared via General Procedure C using benzylic boronate 2a. The crude mixture was purified by silica gel chromatography (20\% EtOAc/hexanes) to give 3a (run 1 (66 mg of 2a): $38 \mathrm{mg}, 95 \%$ ) as a white solid. The enantiomeric excess was determined to be $99 \%$ (run 1: $99 \%$ ee; run 2: $99 \%$ ee) by chiral 
HPLC analysis (CHIRAPAK IA, $1.0 \mathrm{~mL} / \mathrm{min}, 1 \% i$-PrOH/hexanes, $\lambda=254 \mathrm{~nm}$ ); $\mathrm{t}_{\mathrm{R}}$ (major) $=43.70 \mathrm{~min}, \mathrm{t}_{\mathrm{R}}($ minor $\left.)=45.74 \mathrm{~min}\right) ;{ }^{1} \mathrm{H} \mathrm{NMR}\left(400 \mathrm{MHz}, \mathrm{CDCl}_{3}\right) \delta 7.89-7.79(\mathrm{~m}, 4 \mathrm{H})$, $7.56-7.42(\mathrm{~m}, 3 \mathrm{H}), 5.06$ (q, $J=6.2 \mathrm{~Hz}, 1 \mathrm{H}), 2.07(\mathrm{~s}, 1 \mathrm{H}), 1.58(\mathrm{~d}, J=6.4 \mathrm{~Hz}, 3 \mathrm{H}) ;{ }^{13} \mathrm{C}$ NMR $\left(101 \mathrm{MHz}, \mathrm{CDCl}_{3}\right) \delta 143.3,133.4,133.0,128.5,128.1,127.8,126.3,126.0$, $123.97,123.9570 .7,25.3$. The spectral data match that previously reported in the literature. $^{6}$

The absolute configuration of alcohol 3a was determined to be $R$ by comparison of its HPLC trace to that of commercially available, enantioenriched 3a.

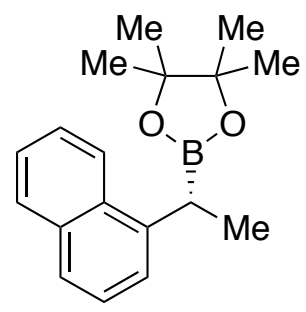

$(R)-4,4,5,5$-tetramethyl-2-(1-(naphthalen-1-yl)ethyl)-1,3,2-dioxaborolane $\quad((R)$ 2b). Prepared via General Procedure A using ammonium salt 1b (amine purchased in $>99 \%$ ee). The crude mixture was purified by silica gel chromatography (5\% EtOAc/hexanes) to give $\mathbf{2 b}$ (run 1: $47 \mathrm{mg}, 56 \%$; run 2: $47 \mathrm{mg}, 56 \%$ ) as a white solid: ${ }^{1} \mathrm{H}$ NMR (400 MHz, $\left.\mathrm{CDCl}_{3}\right) \delta 8.13(\mathrm{~d}, J=8.2 \mathrm{~Hz}, 1 \mathrm{H}), 7.88-7.83(\mathrm{~m}, 1 \mathrm{H}), 7.69(\mathrm{~d}, J=7.5$ Hz, 1H), $7.53-7.39$ (m, 4H), 3.14 (q, $J=7.4 \mathrm{~Hz}, 1 \mathrm{H}), 1.52$ (d, $J=7.5 \mathrm{~Hz}, 3 \mathrm{H}), 1.23$ (s, $6 \mathrm{H}), 1.22(\mathrm{~s}, 6 \mathrm{H}) ;{ }^{13} \mathrm{C} \mathrm{NMR}\left(101 \mathrm{MHz}, \mathrm{CDCl}_{3}\right) \delta 141.5,134.0,132.1,128.9,126.0$, $125.5,125.4,124.4,124.2,83.6,24.8,24.7,16.6 .{ }^{4}$ The spectral data matches that previously reported in the literature. ${ }^{5}$

Boronate 2b was oxidized to alcohol $\mathbf{3 b}$ via General Procedure C. The enantiomeric excess was determined to be $92 \%$ (run 1: $92 \%$, run 2: $91 \%$ ) by chiral HPLC analysis. See alcohol $\mathbf{3 b}$ below. 


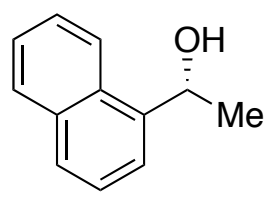

(R)-1-(naphthalen-1-yl)ethanol (3b). Prepared via General Procedure C using benzylic boronate $\mathbf{2 b}$. The crude mixture was purified by silica gel chromatography $(20 \%$ EtOAc/hexanes) to give $\mathbf{3 b}$ (run 1 (47 $\mathrm{mg}$ of $\mathbf{2 b}$ ): $21 \mathrm{mg}, 72 \%$ ) as a colorless oil. The enantiomeric excess was determined to be $92 \%$ (run 1: $92 \%$ ee; run 2: $91 \%$ ee) by chiral HPLC analysis (CHIRAPAK IC, $1.0 \mathrm{~mL} / \mathrm{min}, 2 \% i$-PrOH/hexanes, $\lambda=254 \mathrm{~nm}$ ); $\mathrm{t}_{\mathrm{R}}$ (major) $=23.87 \mathrm{~min}, \mathrm{t}_{\mathrm{R}}($ minor $\left.)=18.43 \mathrm{~min}\right):{ }^{1} \mathrm{H} \mathrm{NMR}\left(400 \mathrm{MHz}, \mathrm{CDCl}_{3}\right) \delta 8.12(\mathrm{~d}, J=8.2 \mathrm{~Hz}$, 1H), 7.89 (d, $J=7.6 \mathrm{~Hz}, 1 \mathrm{H}), 7.79$ (d, $J=8.2 \mathrm{~Hz}, 1 \mathrm{H}), 7.69$ (d, $J=7.1 \mathrm{~Hz}, 1 \mathrm{H}), 7.57-$ $7.45(\mathrm{~m}, 3 \mathrm{H}), 5.69(\mathrm{q}, J=6.3 \mathrm{~Hz}, 1 \mathrm{H}), 1.96(\mathrm{~s}, 1 \mathrm{H}), 1.68(\mathrm{~d}, J=6.5 \mathrm{~Hz}, 3 \mathrm{H}) ;{ }^{13} \mathrm{C} \mathrm{NMR}$ $\left(101 \mathrm{MHz}, \mathrm{CDCl}_{3}\right) \delta 141.5,133.9,130.4,129.1,128.1,126.2,125.73,125.70,123.3$, $122.1,67.3,24.5$. The spectral data of this compound match that previously reported in the literature. ${ }^{6}$

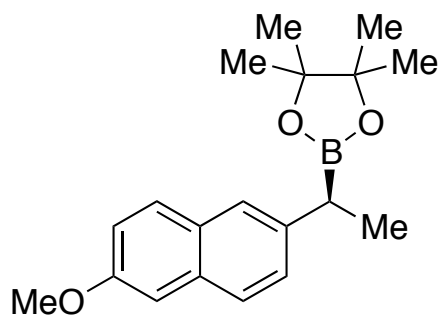

(S)-2-(1-(6-methoxynaphthalen-2-yl)ethyl)-4,4,5,5-tetramethyl-1,3,2-dioxaborolane

(2c). Prepared via General Procedure A using ammonium salt 1c (amine prepared in $\geq 95 \%$ ee). The crude mixture was purified by silica gel chromatography (5\% EtOAc/hexanes) to give $2 \mathrm{c}$ (run 1: $78 \mathrm{mg}, 83 \%$; run 2: $78 \mathrm{mg}, 83 \%$ ) as a white solid: ${ }^{1} \mathrm{H}$ NMR (400 MHz, $\left.\mathrm{CDCl}_{3}\right) \delta 7.68(\mathrm{t}, J=8.1 \mathrm{~Hz}, 2 \mathrm{H}), 7.60$ (s, 1H), $7.38(\mathrm{dd}, J=8.4,1.4$ $\mathrm{Hz}, 1 \mathrm{H}), 7.12$ (d, $J=8.1 \mathrm{~Hz}, 2 \mathrm{H}), 3.91$ (s, 3H), 2.60 (q, $J=7.5 \mathrm{~Hz}, 1 \mathrm{H}), 1.44$ (d, $J=7.5$ $\mathrm{Hz}, 3 \mathrm{H}), 1.23$ (s, 6H), 1.22 (s, 6H); ${ }^{13} \mathrm{C}$ NMR (101 MHz, $\left.\mathrm{CDCl}_{3}\right) \delta$ 157.0, 140.3, 132.7, $129.5,129.1,127.8,126.7,125.3,118.5,105.7,83.5,55.4,24.8,24.7,17.1{ }^{4}$ The spectral data match that previously reported in the literature. ${ }^{7}$ 
Boronate 2c was oxidized to alcohol 3c via General Procedure C. The enantiomeric excess was determined to be $99 \%$ (run 1: $98 \%$ ee; run 2: $99 \%$ ee) by chiral HPLC analysis. See alcohol 3c below.<smiles>COc1ccc2cc([C@@H](C)O)ccc2c1</smiles>

(S)-1-(6-methoxynaphthalen-2-yl)ethanol (3c). Prepared via General Procedure C using benzylic boronate 2c. The crude mixture was purified by silica gel chromatography (20\% EtOAc/hexanes) to give 3c (run 1 (67 $\mathrm{mg}$ of $2 \mathrm{c}$ ): $31 \mathrm{mg}, 72 \%$ ) as a white solid; the enantiomeric excess was determined to be $99 \%$ (run 1: $98 \%$ ee; run 2: $99 \%$ ee) by chiral HPLC analysis (CHIRAPAK IB, $1.0 \mathrm{~mL} / \mathrm{min}, 3 \% i-\mathrm{PrOH} /$ hexanes, $\lambda=254 \mathrm{~nm}$ ); $\mathrm{t}_{\mathrm{R}}$ (major) $=19.99 \mathrm{~min}, \mathrm{t}_{\mathrm{R}}($ minor $\left.)=25.84 \mathrm{~min}\right):{ }^{1} \mathrm{H} \mathrm{NMR}\left(600 \mathrm{MHz}, \mathrm{CDCl}_{3}\right) \delta 7.75-7.69(\mathrm{~m}, 3 \mathrm{H})$, $7.47(\mathrm{~d}, J=8.5 \mathrm{~Hz}, 1 \mathrm{H}), 7.18-7.11(\mathrm{~m}, 2 \mathrm{H}), 5.02(\mathrm{q}, J=6.5 \mathrm{~Hz}, 1 \mathrm{H}), 3.92(\mathrm{~s}, 3 \mathrm{H}), 2.03$ $(\mathrm{s}, 1 \mathrm{H}), 1.57(\mathrm{~d}, J=6.5 \mathrm{~Hz}, 3 \mathrm{H}) ;{ }^{13} \mathrm{C} \mathrm{NMR}\left(151 \mathrm{MHz}, \mathrm{CDCl}_{3}\right) \delta 157.8,141.1,134.2$, $129.6,128.9,127.3,124.5,123.9,119.1,105.9,70.6,55.5,25.2$. The spectral data match that previously reported in the literature. ${ }^{8}$

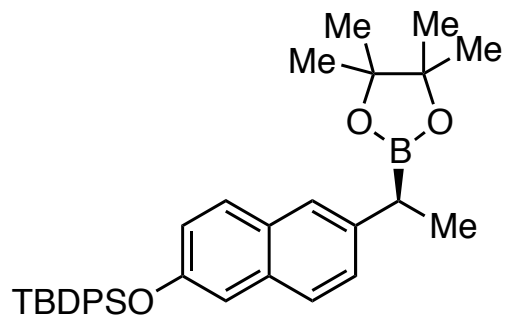

\section{(S)-tert-butyldiphenyl((6-(1-(4,4,5,5-tetramethyl-1,3,2-dioxaborolan-2-}

yl)ethyl)naphthalen-2-yl)oxy)silane (2d). Prepared via General Procedure A using ammonium salt 1d (amine prepared in $\geq 95 \%$ ee). The crude mixture was purified by silica gel chromatography (5\% EtOAc/hexanes) to give $\mathbf{2 d}$ (76 $\mathrm{mg}, 47 \%$ ) as a white solid $\left(\mathrm{mp} \mathrm{84-86}{ }^{\circ} \mathrm{C}\right):{ }^{1} \mathrm{H}$ NMR $\left(400 \mathrm{MHz}, \mathrm{CDCl}_{3}\right) \delta 7.85-7.78(\mathrm{~m}, 4 \mathrm{H}), 7.62-7.53(\mathrm{~m}, 2 \mathrm{H})$, 
$7.49-7.37(\mathrm{~m}, 7 \mathrm{H}), 7.29$ (dd, $J=8.5,1.5 \mathrm{~Hz}, 1 \mathrm{H}), 7.10-7.03(\mathrm{~m}, 2 \mathrm{H}), 2.57$ (q, $J=7.4$ $\mathrm{Hz}, 1 \mathrm{H}), 1.42$ (d, $J=7.5 \mathrm{~Hz}, 3 \mathrm{H}), 1.25$ (s, 6H), 1.23 (s, 6H), 1.18 (s, 9H); ${ }^{13} \mathrm{C}$ NMR (101 $\left.\mathrm{MHz}, \mathrm{CDCl}_{3}\right) \delta 152.8,140.4,135.7,133.2,132.7,130.0,129.6,128.8,128.0,127.6$, $126.8,125.2,121.5,114.5,83.5,26.8,24.82,24.79,19.7,17.2 ;{ }^{4}{ }^{11} \mathrm{~B}$ NMR $(193 \mathrm{MHz}$, $\left.\mathrm{CDCl}_{3}\right) \delta 33.6 ;{ }^{29} \mathrm{Si} \mathrm{NMR}\left(79 \mathrm{MHz}, \mathrm{CDCl}_{3}\right) \delta$-6.4; FTIR (neat) 2960, 2858, 1603, 1500, $1352,1143,975,701 \mathrm{~cm}^{-1}$; HRMS (LIFDI) calculated for $\mathrm{C}_{34} \mathrm{H}_{41} \mathrm{BO}_{3} \mathrm{Si}: 536.2887$, found: 536.2894 .

Boronate 2d was oxidized to alcohol 3d via General Procedure C. The enantiomeric excess was determined to be $92 \%$ by chiral HPLC analysis. See alcohol 3d below.

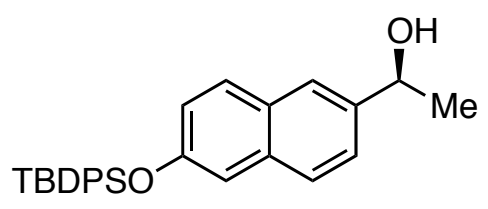

(S)-1-(6-((tert-butyldiphenylsilyl)oxy)naphthalen-2-yl)ethanol (3d). Prepared via General Procedure $\mathrm{C}$ using benzylic boronate 2d. The crude mixture was purified by silica gel chromatography (20\% EtOAc/hexanes) to give 3d (run 1 (71 mg of 2d): $54 \mathrm{mg}$, $95 \%$ ) as a colorless semi-solid. The enantiomeric excess was determined to be $92 \%$ by chiral HPLC analysis (CHIRALPAK IA, $1.0 \mathrm{~mL} / \mathrm{min}, 1 \% i$-PrOH/hexanes, $\lambda=254 \mathrm{~nm}$ ); $\mathrm{t}_{\mathrm{R}}($ major $)=35.70 \mathrm{~min}, \mathrm{t}_{\mathrm{R}}($ minor $)=33.76 \mathrm{~min} .[\alpha]_{\mathrm{D}}{ }^{24}=-20.2^{\circ}\left(\mathrm{c} 2.2, \mathrm{CHCl}_{3}\right):{ }^{1} \mathrm{H} \mathrm{NMR}$ $\left(400 \mathrm{MHz}, \mathrm{CDCl}_{3}\right) \delta 7.83-7.75(\mathrm{~m}, 4 \mathrm{H}), 7.67(\mathrm{~s}, 1 \mathrm{H}), 7.63-7.59(\mathrm{~m}, 1 \mathrm{H}), 7.50(\mathrm{~d}, J=$ $8.5 \mathrm{~Hz}, 1 \mathrm{H}), 7.47-7.34$ (m, 7H), $7.12-7.05$ (m, 2H), 4.98 (q, $J=6.4 \mathrm{~Hz}, 1 \mathrm{H}), 1.99$ (bs, $1 \mathrm{H}), 1.54(\mathrm{~d}, J=6.5 \mathrm{~Hz}, 3 \mathrm{H}), 1.16(\mathrm{~s}, 9 \mathrm{H}) ;{ }^{13} \mathrm{C} \mathrm{NMR}\left(101 \mathrm{MHz}, \mathrm{CDCl}_{3}\right) \delta$ 153.6, 141.1, 135.7, 134.0, 133.0, 130.1, 129.3, 128.9, 128.0, 127.3, 124.2, 123.7, 122.0, 114.7, 70.6, 26.7, 25.2, 19.7; ${ }^{29} \mathrm{Si} \mathrm{NMR}\left(119 \mathrm{MHz}, \mathrm{CDCl}_{3}\right) \delta$-5.9; FTIR (neat) 3347 (broad), 3051, 2931, 2858, 1606, 1482, 1263, 1175, 114, 76, 701, $504 \mathrm{~cm}^{-1}$; HRMS (CI+) calculated for $\mathrm{C}_{28} \mathrm{H}_{30} \mathrm{BO}_{2} \mathrm{Si}: 427.2093$, found: 427.2090 . 


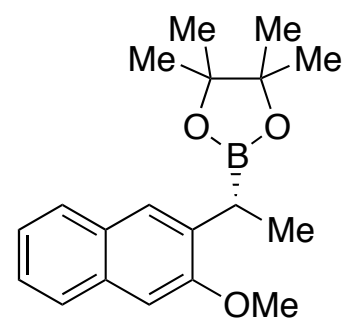

(R)-2-(1-(3-methoxynaphthalen-2-yl)ethyl)-4,4,5,5-tetramethyl-1,3,2-dioxaborolane

(2e). Prepared via General Procedure A using ammonium salt 1e (amine prepared in $\geq 95 \%$ ee). The crude mixture was purified by silica gel chromatography $(5 \%$ EtOAc/hexanes) to give 2e (46 mg, 49\%) as an opaque semi-solid: ${ }^{1} \mathrm{H}$ NMR (400 MHz, $\left.\mathrm{CDCl}_{3}\right) \delta 7.77-7.69(\mathrm{~m}, 2 \mathrm{H}), 7.60(\mathrm{~s}, 1 \mathrm{H}), 7.38(\mathrm{ddd}, J=8.1,6.8,1.4 \mathrm{~Hz}, 1 \mathrm{H}), 7.31$ (ddd, $J=8.1,6.9,1.3 \mathrm{~Hz}, 1 \mathrm{H}), 7.08(\mathrm{~s}, 1 \mathrm{H}), 3.93$ (s, 3H), 2.63 (q, $J=7.5 \mathrm{~Hz}, 1 \mathrm{H}), 1.43$ $(\mathrm{d}, J=7.5 \mathrm{~Hz}, 3 \mathrm{H}), 1.26(\mathrm{~s}, 12 \mathrm{H}) ;{ }^{13} \mathrm{C} \mathrm{NMR}\left(101 \mathrm{MHz}, \mathrm{CDCl}_{3}\right) \delta 156.2,135.7,133.1$, $129.4,127.3,126.5,126.3,125.3,123.5,104.4,83.2,55.1,24.80,24.77,14.8 ;{ }^{411} \mathrm{~B} \mathrm{NMR}$ $\left(193 \mathrm{MHz}, \mathrm{CDCl}_{3}\right) \delta 33.6$; FTIR (neat) 2976, 1472, 1388, 1251, 1144, 847, $746 \mathrm{~cm}^{-1}$; HRMS (LIFDI) calculated for $\mathrm{C}_{19} \mathrm{H}_{25} \mathrm{BO}_{3}: 312.1897$, found: 312.1884 .

Boronate 2e was oxidized to alcohol 3e via General Procedure C. The enantiomeric excess was determined to be $95 \%$ by chiral HPLC analysis. See alcohol $3 \mathbf{e}$ below.

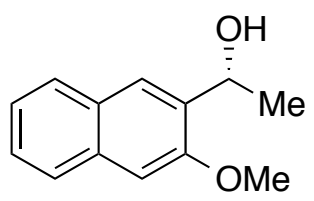

(R)-1-(3-methoxynaphthalen-2-yl)ethanol (3e). Prepared via General Procedure C using benzylic boronate 2e. The crude mixture was purified by silica gel chromatography (20\% EtOAc/hexanes) to give 3e (run 1 (46 mg of 2e): $25 \mathrm{mg}, 83 \%$ ) as a clear oil. The enantiomeric excess was determined to be 95\% (CHIRALPAK IB, $1.0 \mathrm{~mL} / \mathrm{min}, 5 \% i$ $\mathrm{PrOH} /$ hexanes, $\lambda=254 \mathrm{~nm}) ; \mathrm{t}_{\mathrm{R}}($ major $)=22.47 \mathrm{~min}, \mathrm{t}_{\mathrm{R}}($ minor $)=14.96 \mathrm{~min} .[\alpha]_{\mathrm{D}}{ }^{24}=-35.7^{\circ}$ (c $\left.0.11, \mathrm{CHCl}_{3}\right):{ }^{1} \mathrm{H} \mathrm{NMR}\left(600 \mathrm{MHz}, \mathrm{CDCl}_{3}\right) \delta 7.80-7.76(\mathrm{~m}, 2 \mathrm{H}), 7.73(\mathrm{~d}, J=8.2 \mathrm{~Hz}$, $1 \mathrm{H}), 7.44(\mathrm{t}, J=7.5 \mathrm{~Hz}, 1 \mathrm{H}), 7.35$ (t, $J=7.9 \mathrm{~Hz}, 1 \mathrm{H}), 7.13(\mathrm{~s}, 1 \mathrm{H}), 5.22(\mathrm{q}, J=6.0 \mathrm{~Hz}$, 
1H), $3.97(\mathrm{~s}, 3 \mathrm{H}), 2.77(\mathrm{~s}, 1 \mathrm{H}), 1.61(\mathrm{~d}, J=6.6 \mathrm{~Hz}, 3 \mathrm{H}) ;{ }^{13} \mathrm{C} \mathrm{NMR}\left(151 \mathrm{MHz}, \mathrm{CDCl}_{3}\right) \delta$ $155.6,135.0,133.9,128.9,127.9,126.5,126.4,125.3,124.1,105.6,67.0,55.5,23.1$. The spectral data match that previously reported in the literature for the racemic compound. ${ }^{9}$

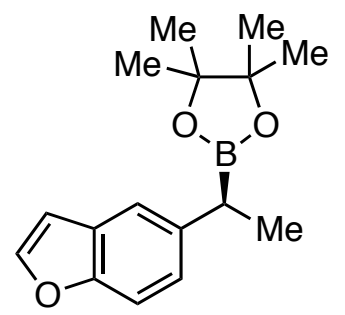

(S)-2-(1-(benzofuran-5-yl)ethyl)-4,4,5,5-tetramethyl-1,3,2-dioxaborolane (2f).

Prepared via General Procedure A, except that the reaction temperature was $50^{\circ} \mathrm{C}$, using ammonium salt $1 \mathbf{f}$ (amine prepared in $\geq 95 \%$ ee). The crude mixture was purified by silica gel chromatography (5\% EtOAc/hexanes) to give 2 f (run 1: $48 \mathrm{mg}, 59 \%$; run 2: $55 \mathrm{mg}$, $67 \%$ ) as a white solid (mp 58-59 $\left.{ }^{\circ} \mathrm{C}\right):{ }^{1} \mathrm{H}$ NMR $\left(400 \mathrm{MHz}, \mathrm{CDCl}_{3}\right) \delta 7.57(\mathrm{~d}, J=2.2 \mathrm{~Hz}$, $1 \mathrm{H}), 7.45$ (d, $J=1.8 \mathrm{~Hz}, 1 \mathrm{H}), 7.41$ (d, $J=8.5 \mathrm{~Hz}, 1 \mathrm{H}), 7.17$ (dd, $J=8.6,1.9 \mathrm{~Hz}, 1 \mathrm{H}$ ), 6.71 (dd, $J=2.2,1.0 \mathrm{~Hz}, 1 \mathrm{H}), 2.54$ (q, $J=7.5 \mathrm{~Hz}, 1 \mathrm{H}), 1.39$ (d, $J=7.5 \mathrm{~Hz}, 3 \mathrm{H}), 1.23$ (s, $6 \mathrm{H}), 1.21(\mathrm{~s}, 6 \mathrm{H}) ;{ }^{13} \mathrm{C}$ NMR $\left(101 \mathrm{MHz}, \mathrm{CDCl}_{3}\right) \delta$ 153.4, 144.9, 139.6, 127.7, 124.7, 119.8, 111.1, 106.7, 83.4, 24.79, 24.75, 17.9; ${ }^{411} \mathrm{~B}$ NMR (193 MHz, $\left.\mathrm{CDCl}_{3}\right) \delta$ 33.6; FTIR (neat) $2976,1467,1319,1144,843,737 \mathrm{~cm}^{-1}$; HRMS (LIFDI) calculated for $\mathrm{C}_{16} \mathrm{H}_{21} \mathrm{BO}_{3}$ : 272.1584, found: 272.1611 .

Boronate 2f was oxidized to alcohol 3f via General Procedure C. The enantiomeric excess was determined to be $98 \%$ (run 1: $97 \%$ ee; run 2: $98 \%$ ee) by chiral HPLC analysis. See alcohol $\mathbf{3 f}$ below.<smiles>CC(O)c1ccc2occc2c1</smiles> 
(S)-1-(benzofuran-5-yl)ethanol (3f). Prepared via General Procedure C using benzylic boronate 2f. The crude mixture was purified by silica gel chromatography $(20 \%$ EtOAc/hexanes) to give $\mathbf{3 f}$ (run 1 (41 $\mathrm{mg}$ of $\mathbf{2 f}$ ): $15 \mathrm{mg}, 61 \%$ ) as a clear oil. The enantiomeric excess was determined to be $98 \%$ (run 1: $97 \%$ ee; run 2: $98 \%$ ee) by chiral HPLC analysis (CHIRALPAK IC, $0.5 \mathrm{~mL} / \mathrm{min}, 2 \% i$-PrOH/hexanes, $\lambda=254 \mathrm{~nm}$ ); $\mathrm{t}_{\mathrm{R}}($ major $)=45.76 \mathrm{~min}, \mathrm{t}_{\mathrm{R}}($ minor $)=43.97 \mathrm{~min} .[\alpha]_{\mathrm{D}}{ }^{24}=-33.0^{\circ}\left(\mathrm{c} 0.79, \mathrm{CHCl}_{3}\right):{ }^{1} \mathrm{H} \mathrm{NMR}$ $\left(400 \mathrm{MHz}, \mathrm{CDCl}_{3}\right) \delta 7.67-7.57(\mathrm{~m}, 2 \mathrm{H}), 7.48(\mathrm{~d}, J=8.5 \mathrm{~Hz}, 1 \mathrm{H}), 7.32(\mathrm{dd}, J=8.6,1.8$ $\mathrm{Hz}, 1 \mathrm{H}), 6.76(\mathrm{~d}, J=1.1 \mathrm{~Hz}, 1 \mathrm{H}), 5.01(\mathrm{q}, J=6.4 \mathrm{~Hz}, 1 \mathrm{H}), 1.92$ (bs, $1 \mathrm{H}), 1.54$ (d, $J=6.4$ $\mathrm{Hz}, 3 \mathrm{H}) ;{ }^{13} \mathrm{C} \mathrm{NMR}\left(101 \mathrm{MHz}, \mathrm{CDCl}_{3}\right) \delta 154.5,145.6,140.7,127.6,122.2,118.1,111.5$, 106.8, 70.8, 25.7; FTIR (neat) 3344 (broad), 2921, 1444, 1261, 1129, 1072, 891, 813, 738 $\mathrm{cm}^{-1}$; HRMS (CI+) calculated for $\mathrm{C}_{10} \mathrm{H}_{11} \mathrm{O}_{2}: 163.0759$, found: 163.0756 .

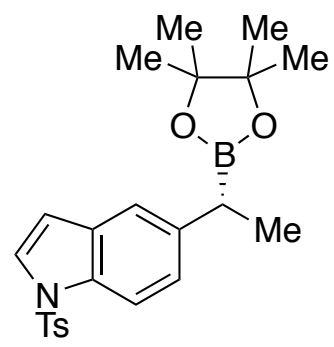

(R)-5-(1-(4,4,5,5-tetramethyl-1,3,2-dioxaborolan-2-yl)ethyl)-1-tosyl-1 $H$-indole $\quad(2 \mathrm{~g})$.

Prepared via General Procedure A using ammonium salt 1g (prepared in $\geq 95 \%$ ee). Instead of filtering through Celite ${ }^{\circledR}$, the diluted mixture was filtered through a small plug of silica gel and then concentrated. 1,3,5-Trimethoxybenzene was added as internal standard, and the yield was determined by ${ }^{1} \mathrm{H}$ NMR to be $44 \%$ (run 1: $46 \%$, run 2: $41 \%$ ). The reaction mixture was complicated, preventing effective purification and isolation on scale. However, an analytical sample of $\mathbf{2 g}$ (contaminated with $\sim 15 \% \mathrm{~B}_{2} \mathrm{pin}_{2}$ ) was purified by silica gel chromatography (prep TLC, 30\% EtOAc/hexanes) to enable characterization: ${ }^{1} \mathrm{H}$ NMR $\left(600 \mathrm{MHz}, \mathrm{CDCl}_{3}\right) \delta 7.85(\mathrm{~d}, J=8.6 \mathrm{~Hz}, 1 \mathrm{H}), 7.75(\mathrm{~d}, J=8.3$ Hz, 2H), 7.49 (d, $J=3.6 \mathrm{~Hz}, 1 \mathrm{H}), 7.34$ (s, 1H), 7.20 (d, $J=8.1 \mathrm{~Hz}, 2 \mathrm{H}), 7.17$ (dd, $J=8.6$, $1.3 \mathrm{~Hz}, 1 \mathrm{H}), 6.57$ (d, $J=3.6 \mathrm{~Hz}, 1 \mathrm{H}), 2.48$ (q, $J=7.5 \mathrm{~Hz}, 1 \mathrm{H}), 2.33$ (s, 3H), 1.32 (d, $J=$ $7.5 \mathrm{~Hz}, 3 \mathrm{H}), 1.20(\mathrm{~s}, 6 \mathrm{H}), 1.18(\mathrm{~s}, 6 \mathrm{H}) ;{ }^{13} \mathrm{C} \mathrm{NMR}\left(101 \mathrm{MHz}, \mathrm{CDCl}_{3}\right) \delta 144.9,140.2$, 135.6, 133.1, 131.2, 130.0, 127.0, 126.3, 125.2, 120.0, 113.4, 109.3, 83.5, 24.79, 24.76, 
21.7, 17.6; ${ }^{411} \mathrm{~B}\left(193 \mathrm{MHz}, \mathrm{CDCl}_{3}\right) \delta$ 33.8; FTIR (neat, $\left.\mathrm{cm}^{-1}\right)$ 2977, 2930, 1459, 1372, 1173, 676, 583; HRMS (CI) calculated for $\mathrm{C}_{23} \mathrm{H}_{28} \mathrm{BNO}_{4} \mathrm{~S}: 425.1832$, found: 425.1840 .

The sample was then concentrated and subjected to General Procedure $\mathrm{C}$ for oxidation and determination of enantiomeric excess.

Boronate $\mathbf{2 g}$ was oxidized to alcohol $\mathbf{3 g}$ via General Procedure C. The enantiomeric excess was determined to be $96 \%$ by chiral HPLC analysis. See alcohol $\mathbf{3 g}$ below.

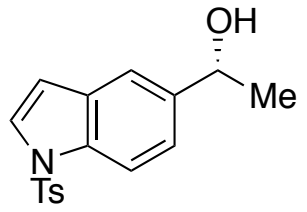

(R)-1-(1-tosyl-1 $H$-indol-5-yl)ethanol (3g). Prepared via General Procedure C using benzylic boronate $2 \mathrm{~g}$. The crude mixture was purified by silica gel chromatography ( $40 \%$ EtOAc/hexanes) to give $\mathbf{3 g}$ (run 1 (43 $\mathrm{mg}$ of $\mathbf{2 g}$ ): $34 \mathrm{mg}$, 79\%) as a pale yellow semisolid. The enantiomeric excess was determined to be $96 \%$ (run 1: $96 \%$ ee; run 2: $95 \%$ ee) by chiral HPLC analysis (CHIRALPAK IA, $1.0 \mathrm{~mL} / \mathrm{min}, 5 \% i$-PrOH/hexanes, $\lambda=254$ $\mathrm{nm}) ; \mathrm{t}_{\mathrm{R}}($ major $)=58.15 \mathrm{~min}, \mathrm{t}_{\mathrm{R}}($ minor $)=53.38 \mathrm{~min} .[\alpha]_{\mathrm{D}}{ }^{24}=-18.7^{\circ}\left(\mathrm{c} 0.165, \mathrm{CHCl}_{3}\right):{ }^{1} \mathrm{H}$ NMR (400 MHz, CDCl $) \delta 7.94(\mathrm{~d}, J=8.6 \mathrm{~Hz}, 1 \mathrm{H}), 7.75(\mathrm{~d}, J=8.4 \mathrm{~Hz}, 2 \mathrm{H}), 7.55(\mathrm{~d}, J=$ $3.6 \mathrm{~Hz}, 1 \mathrm{H}), 7.52(\mathrm{~s}, 1 \mathrm{H}), 7.31$ (dd, $J=8.6,1.5 \mathrm{~Hz}, 1 \mathrm{H}), 7.20(\mathrm{~d}, J=8.2 \mathrm{~Hz}, 2 \mathrm{H}), 6.62(\mathrm{~d}$, $J=3.6 \mathrm{~Hz}, 1 \mathrm{H}$ ), 4.94 (q, $J=6.4 \mathrm{~Hz}, 1 \mathrm{H}), 2.32$ (s, 3H), 1.99 (s, 1H), 1.49 (d, $J=6.4 \mathrm{~Hz}$, $3 \mathrm{H}) ;{ }^{13} \mathrm{C} \mathrm{NMR}\left(101 \mathrm{MHz}, \mathrm{CDCl}_{3}\right) \delta 145.1,141.2,135.3,134.3,131.0,130.0,126.9$, 126.9, 122.5, 118.2 , 113.7, 109.2, 70.6, 25.5, 21.7; FTIR (neat) 3379 (broad), 2971, 1596, $1369,1173,1128,676,579 \mathrm{~cm}^{-1}$; HRMS $(\mathrm{CI}+)[\mathrm{M}+\mathrm{H}]^{+}$calculated for $\left[\mathrm{C}_{17} \mathrm{H}_{18} \mathrm{NO}_{3} \mathrm{~S}\right]^{+}$: 316.1007, found: 316.1017 . 


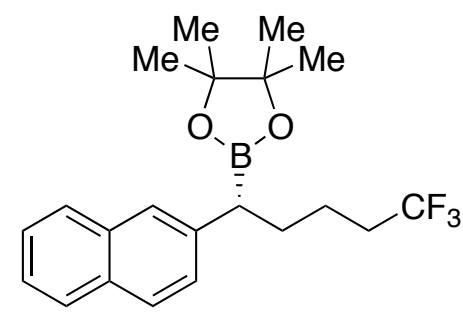

\section{(R)-4,4,5,5-tetramethyl-2-(5,5,5-trifluoro-1-(naphthalen-2-yl)pentyl)-1,3,2-}

dioxaborolane (2h). Prepared via General Procedure A using ammonium salt 1h (amine prepared in $\geq 95 \%$ ee). The crude mixture was purified by silica gel chromatography (5\% EtOAc/hexanes) to give $\mathbf{2 h}$ (run 1: $73 \mathrm{mg}$, 64\%; run 2: $69 \mathrm{mg}, 60 \%$ ) as a white solid (mp 74-76 ${ }^{\circ} \mathrm{C}$ ); ${ }^{1} \mathrm{H}$ NMR (400 MHz, $\left.\mathrm{CDCl}_{3}\right) \delta 7.86-7.74(\mathrm{~m}, 3 \mathrm{H}), 7.65$ (s, $\left.1 \mathrm{H}\right), 7.50-7.33$ (m, 3H), $2.50(\mathrm{t}, J=7.9 \mathrm{~Hz}, 1 \mathrm{H}), 2.19-1.96(\mathrm{~m}, 3 \mathrm{H}), 1.91-1.78(\mathrm{~m}, 1 \mathrm{H}), 1.65-1.50$ (m, 2H), $1.23(\mathrm{~s}, 6 \mathrm{H}), 1.20(\mathrm{~s}, 6 \mathrm{H}) ;{ }^{13} \mathrm{C} \mathrm{NMR}\left(151 \mathrm{MHz}, \mathrm{CDCl}_{3}\right) \delta 140.2,134.0,132.1$, 128.1, 127.7, 127.6, $127.4\left(\mathrm{q}, J_{\mathrm{C}-\mathrm{F}}=276.4 \mathrm{~Hz}\right), 127.3,126.5,126.0,125.2,83.7,33.9(\mathrm{q}$, $\left.J_{\mathrm{C}-\mathrm{F}}=28.4 \mathrm{~Hz}\right), 31.6,24.8,24.7,21.7\left(\mathrm{q}, J_{\mathrm{C}-\mathrm{F}}=2.7 \mathrm{~Hz}\right) ;{ }^{41} \mathrm{~B} \mathrm{NMR}\left(193 \mathrm{MHz}, \mathrm{CDCl}_{3}\right) \delta$ 33.1; ${ }^{19} \mathrm{~F}$ NMR (376.5 MHz, $\left.\mathrm{CDCl}_{3}\right) \delta$-66.3; FTIR (neat) 2978, 1361, 1259, 1141, 857, $749 \mathrm{~cm}^{-1}$; HRMS (CI+) calculated for $\mathrm{C}_{21} \mathrm{H}_{26} \mathrm{BF}_{3} \mathrm{O}_{2}: 379.2049$, found: 379.2034 .

Boronate $\mathbf{2 h}$ was oxidized to alcohol $\mathbf{3 h}$ via General Procedure C. The enantiomeric excess was determined to be $96 \%$ (run 1: $96 \%$ ee; run 2: $96 \%$ ee) by chiral HPLC analysis. See alcohol $\mathbf{3 h}$ below.

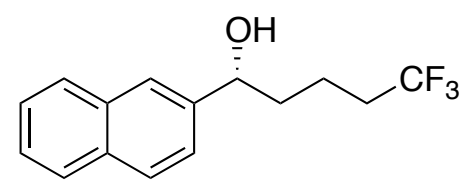

(R)-5,5,5-trifluoro-1-(naphthalen-2-yl)pentan-1-ol (3h). Prepared via General Procedure $\mathrm{C}$ using benzylic boronate $\mathbf{2 h}$. The crude mixture was purified by silica gel chromatography (20\% EtOAc/hexanes) to give $\mathbf{3 h}$ (run 1 (61 $\mathrm{mg}$ of $\mathbf{2 h}$ ): $40 \mathrm{mg}, 93 \%$ ) as a white solid (mp $48-50^{\circ} \mathrm{C}$ ). The enantiomeric excess was determined to be $96 \%$ (run 1 : 96\% ee; run 2: 96\% ee) by chiral HPLC analysis (CHIRALPAK IB, $1.0 \mathrm{~mL} / \mathrm{min}, 3 \% i$ PrOH/hexanes, $\lambda=254 \mathrm{~nm}) ; \mathrm{t}_{\mathrm{R}}($ major $)=28.25 \mathrm{~min}, \mathrm{t}_{\mathrm{R}}($ minor $)=25.09 \mathrm{~min} .[\alpha]_{\mathrm{D}}{ }^{24}=$ 
$+38.4^{\circ}\left(\mathrm{c} 0.75, \mathrm{CHCl}_{3}\right):{ }^{1} \mathrm{H}$ NMR $\left(400 \mathrm{MHz}, \mathrm{CDCl}_{3}\right) \delta 7.89-7.80(\mathrm{~m}, 3 \mathrm{H}), 7.76(\mathrm{~s}, 1 \mathrm{H})$, $7.55-7.48$ (m, 2H), 7.46 (dd, $J=8.6,1.8 \mathrm{~Hz}, 1 \mathrm{H}), 4.87-4.79$ (m, 1H), 2.20 - 2.02 (m, $3 \mathrm{H}), 1.99-1.67(\mathrm{~m}, 3 \mathrm{H}), 1.68-1.52(\mathrm{~m}, 1 \mathrm{H}) ;{ }^{13} \mathrm{C} \mathrm{NMR}\left(101 \mathrm{MHz}, \mathrm{CDCl}_{3}\right) \delta$ 141.7, 133.4, 133.2, 128.7, 128.1, 127.9, $127.2\left(\mathrm{q}, J_{\mathrm{C}-\mathrm{F}}=277.5 \mathrm{~Hz}\right), 126.5,126.2,124.7,123.9$, $74.4,37.8,33.7\left(\mathrm{q}, J_{\mathrm{C}-\mathrm{F}}=28.6 \mathrm{~Hz}\right), 18.6\left(\mathrm{q}, J_{\mathrm{C}-\mathrm{F}}=3.0 \mathrm{~Hz}\right) ;{ }^{19} \mathrm{~F} \mathrm{NMR}\left(376.5 \mathrm{~Hz}, \mathrm{CDCl}_{3}\right)$ $\delta$-66.3; FTIR (neat) 3350 (broad), 2947, 1391, 1259, 1134, 1028, 821, 749, $479 \mathrm{~cm}^{-1}$; HRMS (CI+) calculated for $\mathrm{C}_{15} \mathrm{H}_{16} \mathrm{~F}_{3} \mathrm{O}: 269.1153$, found: 269.1158 .

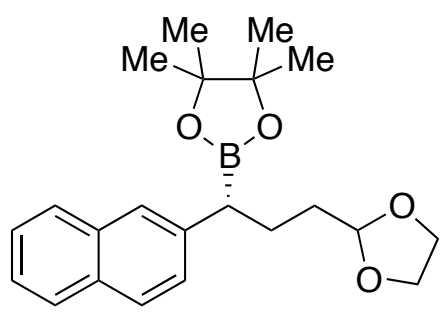

\section{(R)-2-(3-(1,3-dioxolan-2-yl)-1-(naphthalen-2-yl)propyl)-4,4,5,5-tetramethyl-1,3,2-}

dioxaborolane (2i). Prepared via General Procedure A using ammonium salt 1i (amine prepared in $\geq 95 \%$ ee). The crude mixture was purified by silica gel chromatography $\left(10 \%\right.$ EtOAc/hexanes) to give $\mathbf{2 i}(64 \mathrm{mg}, 58 \%)$ as a white solid $\left(\mathrm{mp} 82-84{ }^{\circ} \mathrm{C}\right)$ (note: a 10:1 mixture of product to $\mathrm{B}_{2} \operatorname{pin}_{2}$ was observed): ${ }^{1} \mathrm{H}$ NMR $\left(400 \mathrm{MHz}, \mathrm{CDCl}_{3}\right) \delta 7.81-$ $7.72(\mathrm{~m}, 3 \mathrm{H}), 7.65$ (s, 1H), $7.47-7.36(\mathrm{~m}, 3 \mathrm{H}), 4.86(\mathrm{t}, J=4.8 \mathrm{~Hz}, 1 \mathrm{H}), 3.98-3.77(\mathrm{~m}$, $4 \mathrm{H}), 2.51(\mathrm{t}, J=8.0 \mathrm{~Hz}, 1 \mathrm{H}), 2.15-2.01(\mathrm{~m}, 1 \mathrm{H}), 1.99-1.84(\mathrm{~m}, 1 \mathrm{H}), 1.76-1.59(\mathrm{~m}$, $2 \mathrm{H}), 1.21(\mathrm{~s}, 6 \mathrm{H}), 1.19(\mathrm{~s}, 6 \mathrm{H}) ;{ }^{13} \mathrm{C}$ NMR $\left(101 \mathrm{MHz}, \mathrm{CDCl}_{3}\right) \delta 140.5,133.9,131.9$, $127.9,127.63,127.59127 .5,126.5,125.8,125.0,104.7,83.5,64.9,33.5,26.8,24.8$, 24.7; ${ }^{11} \mathrm{~B}$ NMR (193 MHz, $\mathrm{CDCl}_{3}$ ) $\delta$ 33.2; FTIR (neat) 2977, 2882, 1371, 1324, 1141, $857,750 \mathrm{~cm}^{-1}$; HRMS (LIFDI) calculated for $\mathrm{C}_{22} \mathrm{H}_{29} \mathrm{BO}_{4}: 368.2140$, found: 368.2143 .

Boronate $2 \mathbf{i}$ was oxidized to alcohol 3i via General Procedure C. The enantiomeric excess was determined to be $98 \%$ by chiral HPLC analysis. See alcohol $\mathbf{3 i}$ below. 


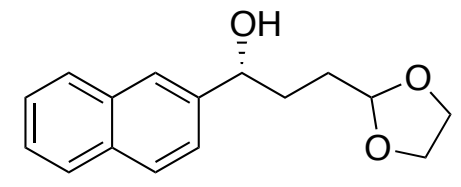

(R)-3-(1,3-dioxolan-2-yl)-1-(naphthalen-2-yl)propan-1-ol (3i). Prepared via General Procedure $\mathrm{C}$ using benzylic boronate $\mathbf{2 i}$. The crude mixture was purified by silica gel chromatography (40\% EtOAc/hexanes) to give $3 \mathbf{i}(36 \mathrm{mg}, 84 \%)$ as a white solid (mp 67$69{ }^{\circ} \mathrm{C}$ ). The enantiomeric excess was determined to be $98 \%$ by chiral HPLC analysis (CHIRALPAK IB, $1.0 \mathrm{~mL} / \mathrm{min}, 5 \% \mathrm{i}-\mathrm{PrOH} /$ hexanes, $\lambda=254 \mathrm{~nm}$ ); $\mathrm{t}_{\mathrm{R}}$ (major) $=31.06 \mathrm{~min}$, $\mathrm{t}_{\mathrm{R}}($ minor $)=27.49 \mathrm{~min} .[\alpha]_{\mathrm{D}}{ }^{24}=-18.4^{\circ}\left(\mathrm{c} 1.78, \mathrm{CHCl}_{3}\right):{ }^{1} \mathrm{H}$ NMR $\left(400 \mathrm{MHz}, \mathrm{CDCl}_{3}\right) \delta$ $7.87-7.78(\mathrm{~m}, 4 \mathrm{H}), 7.53-7.40(\mathrm{~m}, 3 \mathrm{H}), 4.96-4.85(\mathrm{~m}, 2 \mathrm{H}), 4.03-3.81(\mathrm{~m}, 4 \mathrm{H}), 2.74$ $(\mathrm{d}, J=3.5 \mathrm{~Hz}, 1 \mathrm{H}), 2.05-1.92(\mathrm{~m}, 2 \mathrm{H}), 1.91-1.73(\mathrm{~m}, 2 \mathrm{H}) ;{ }^{13} \mathrm{C}$ NMR $(101 \mathrm{MHz}$, $\left.\mathrm{CDCl}_{3}\right) \delta 142.1,133.4,133.0,128.4,128.1,127.8,126.2,125.9,124.6,124.2,104.4$, 74.3, 65.2, 65.1, 33.1, 30.1; FTIR (neat) 3434 (broad), 2882, 1409, 1139, 1031, 822, 751, $479 \mathrm{~cm}^{-1}$; HRMS (CI+) calculated for $\mathrm{C}_{16} \mathrm{H}_{18} \mathrm{O}_{3}: 241.1229$, found: 241.1225 .

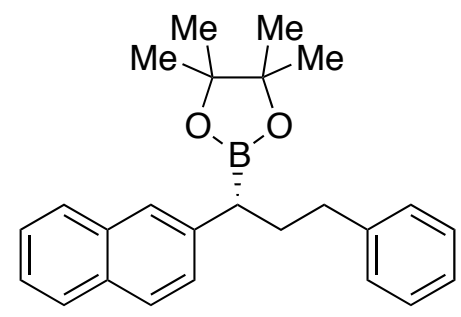

(R)-4,4,5,5-tetramethyl-2-(1-(naphthalen-2-yl)-3-phenylpropyl)-1,3,2-dioxaborolane

(2j). Prepared via General Procedure A using ammonium salt 1j (amine prepared in $\geq 95 \%$ ee). The crude mixture was purified by silica gel chromatography $(5 \%$ EtOAc/hexanes) to give $\mathbf{2 j}$ (80 mg, 72\%) as a white solid ( $\mathrm{mp} 77-79{ }^{\circ} \mathrm{C}$ ): ${ }^{1} \mathrm{H}$ NMR (400 $\left.\mathrm{MHz} \mathrm{CDCl}_{3}\right) \delta 7.87-7.79(\mathrm{~m}, 3 \mathrm{H}), 7.71(\mathrm{~s}, 1 \mathrm{H}), 7.52-7.41(\mathrm{~m}, 3 \mathrm{H}), 7.34-7.28(\mathrm{~m}$, 2H), $7.24-7.18(\mathrm{~m}, 3 \mathrm{H}), 2.65(\mathrm{t}, J=7.9 \mathrm{~Hz}, 2 \mathrm{H}), 2.60$ (t, $J=7.9 \mathrm{~Hz}, 1 \mathrm{H}), 2.37-2.26$ $(\mathrm{m}, 1 \mathrm{H}), 2.21-2.10(\mathrm{~m}, 1 \mathrm{H}), 1.25(\mathrm{~s}, 6 \mathrm{H}), 1.23(\mathrm{~s}, 6 \mathrm{H}) ;{ }^{13} \mathrm{C} \mathrm{NMR}\left(101 \mathrm{MHz}, \mathrm{CDCl}_{3}\right) \delta$ $142.6,140.6,133.9,131.9,128.7,128.4,128.0,127.7,127.6,127.5,126.5,125.84$, 125.81, 125.0, 83.6, 35.6, 34.3, 24.84, 24.76; $\left.{ }^{11} \mathrm{~B} \mathrm{NMR} \mathrm{(193} \mathrm{MHz,} \mathrm{CDCl}_{3}\right) \delta 33.7$; FTIR 
(neat) 2977, 2930, 1323, 1141, 857, 748, $699 \mathrm{~cm}^{-1}$; HRMS (LIFDI) calculated for $\mathrm{C}_{25} \mathrm{H}_{29} \mathrm{BO}_{2}$ : 372.2261, found: 372.2270 .

Boronate $\mathbf{2} \mathbf{j}$ was oxidized to alcohol $\mathbf{3 j}$ via General Procedure C. The enantiomeric excess was determined to be $98 \%$ by chiral HPLC analysis. See alcohol $\mathbf{3 j}$ below.

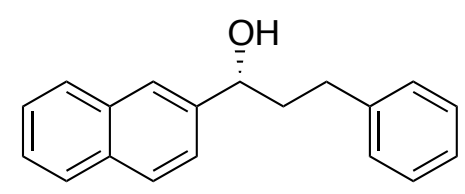

(R)-1-(naphthalen-2-yl)-3-phenylpropan-1-ol (3j). Prepared via General Procedure C using benzylic boronate $\mathbf{2} \mathbf{j}$. The crude mixture was purified by silica gel chromatography (20\% EtOAc/hexanes) to give $\mathbf{3 j}$ ( $43 \mathrm{mg}, 94 \%$ ) as a white solid ( $\mathrm{mp} 85-86{ }^{\circ} \mathrm{C}$ ). The enantiomeric excess was determined to be $98 \%$ by chiral HPLC analysis (CHIRALPAK IA, $1.0 \mathrm{~mL} / \mathrm{min}, 2 \% i-\mathrm{PrOH} /$ hexanes, $\lambda=254 \mathrm{~nm}) ; \mathrm{t}_{\mathrm{R}}($ major $)=35.44 \mathrm{~min}, \mathrm{t}_{\mathrm{R}}($ minor $)=$ 38.33 min: ${ }^{1} \mathrm{H}$ NMR (400 MHz, $\left.\mathrm{CDCl}_{3}\right) \delta 7.89-7.81(\mathrm{~m}, 3 \mathrm{H}), 7.79$ (s, $\left.1 \mathrm{H}\right), 7.55-7.44$ (m, 3H), $7.34-7.27$ (m, 2H), $7.25-7.16(\mathrm{~m}, 3 \mathrm{H}), 4.87$ (ddd, $J=8.1,5.5,2.9 \mathrm{~Hz}, 1 \mathrm{H})$, $2.85-2.65(\mathrm{~m}, 2 \mathrm{H}), 2.30-2.06(\mathrm{~m}, 2 \mathrm{H}), 2.02(\mathrm{~d}, J=3.1 \mathrm{~Hz}, 1 \mathrm{H}) ;{ }^{13} \mathrm{C}$ NMR $(151 \mathrm{MHz}$, $\left.\mathrm{CDCl}_{3}\right) \delta 142.1,141.9,133.5,133.2,128.56,128.59,128.63,128.1,127.9,126.4,126.1$, $126.0,124.9,124.2,74.2,40.5,32.2$. The spectral data match that of the literature. ${ }^{10}$

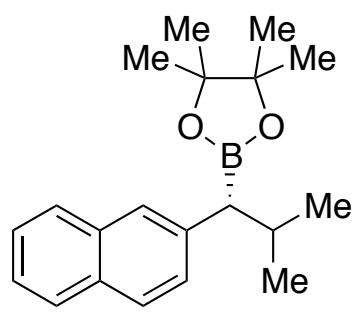

\section{(R)-4,4,5,5-tetramethyl-2-(2-methyl-1-(naphthalen-2-yl)propyl)-1,3,2-dioxaborolane}

(2k). Prepared via General Procedure A using ammonium salt 1k (amine prepared in $\geq 95 \%$ ee). The crude mixture was purified by silica gel chromatography (5\% EtOAc/hexanes) to give $2 \mathbf{k}$ (run 1: $46 \mathrm{mg}$, 49\%; run 2: $47 \mathrm{mg}, 50 \%$ ) as a white solid (mp $\left.85-86{ }^{\circ} \mathrm{C}\right) ;{ }^{1} \mathrm{H}$ NMR $\left(400 \mathrm{MHz}, \mathrm{CDCl}_{3}\right) \delta 7.83-7.71(\mathrm{~m}, 3 \mathrm{H}), 7.66(\mathrm{~s}, 1 \mathrm{H}), 7.47-7.36$ 
(m, 3H), $2.33-2.19(\mathrm{~m}, 1 \mathrm{H}), 2.16(\mathrm{~d}, J=10.5 \mathrm{~Hz}, 1 \mathrm{H}), 1.21(\mathrm{~s}, 6 \mathrm{H}), 1.18(\mathrm{~s}, 6 \mathrm{H}), 1.10$ $(\mathrm{d}, J=6.4 \mathrm{~Hz}, 3 \mathrm{H}), 0.77(\mathrm{~d}, J=6.4 \mathrm{~Hz}, 3 \mathrm{H}) ;{ }^{13} \mathrm{C} \mathrm{NMR}\left(101 \mathrm{MHz}, \mathrm{CDCl}_{3}\right) \delta 140.2$, 133.9, 132.0, 128.1, 127.7, 127.6, 127.3, 125.7, 124.9, 83.4, 31.1, 24.8, 24.7, 23.4, 22.3; ${ }^{4}$ ${ }^{13} \mathrm{C}$ NMR $\left(151 \mathrm{MHz}, \mathrm{C}(\mathrm{O})\left(\mathrm{CD}_{3}\right)_{2}\right) \delta 141.2,134.7,132.9,128.7,128.3,128.3,128.2$, $127.9,126.6,125.7,83.9,31.7,25.0,24.9,23.5,22.4 ;{ }^{4}{ }^{11} \mathrm{~B} \mathrm{NMR}\left(193 \mathrm{MHz}, \mathrm{CDCl}_{3}\right) \delta$ 33.2; FTIR (neat) 2922, 2850, 1382, 1323, 1143, $1103 \mathrm{~cm}^{-1}$; HRMS (LIFDI) calculated for $\mathrm{C}_{20} \mathrm{H}_{27} \mathrm{BO}_{2}: 310.2104$, found: 310.2126 .

Boronate 2k was oxidized to alcohol $\mathbf{3 k}$ via General Procedure C. The enantiomeric excess was determined to be $98 \%$ (run 1: $97 \%$, run 2: $98 \%$ ) by chiral HPLC analysis. See alcohol 3k below.

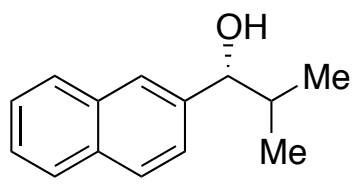

(R)-2-methyl-1-(naphthalen-2-yl)propan-1-ol (3k). Prepared via General Procedure C using benzylic boronate $\mathbf{2 k}$. The crude mixture was purified by silica gel chromatography ( $20 \% \mathrm{EtOAc} /$ hexanes) to give $3 \mathbf{k}$ (run 1 (39 $\mathrm{mg}$ of $\mathbf{2 k}$ ): $7 \mathrm{mg}, 28 \%$ ) as a clear oil. The enantiomeric excess was determined to be $98 \%$ (run 1: $97 \%$, run 2: $98 \%$ ) by chiral HPLC analysis (CHIRALPAK IC, $1.0 \mathrm{~mL} / \mathrm{min}, 2 \% i-\mathrm{PrOH} / \mathrm{hexanes}, \lambda=254 \mathrm{~nm}$ ); $\mathrm{t}_{\mathrm{R}}$ (major) $=$ $17.49 \mathrm{~min}, \mathrm{t}_{\mathrm{R}}$ (minor) $=16.15 \mathrm{~min} ;{ }^{1} \mathrm{H}$ NMR $\left(600 \mathrm{MHz}, \mathrm{CDCl}_{3}\right) \delta 7.86-7.80(\mathrm{~m}, 3 \mathrm{H})$, $7.76(\mathrm{~s}, 1 \mathrm{H}), 7.51-7.44(\mathrm{~m}, 3 \mathrm{H}), 4.54(\mathrm{~d}, J=6.9 \mathrm{~Hz}, 1 \mathrm{H}), 2.12-2.03(\mathrm{~m}, J=6.7 \mathrm{~Hz}$, $1 \mathrm{H}), 1.93(\mathrm{~s}, 1 \mathrm{H}), 1.05(\mathrm{~d}, J=6.7 \mathrm{~Hz}, 3 \mathrm{H}), 0.84(\mathrm{~d}, J=6.8 \mathrm{~Hz}, 3 \mathrm{H}) ;{ }^{13} \mathrm{C} \mathrm{NMR}(101 \mathrm{MHz}$, $\left.\mathrm{CDCl}_{3}\right) \delta 141.2,133.3,133.1,128.2,128.1,127.8,126.2,125.9,125.6,124.8,80.4,35.4$, $19.3,18.4$. The spectral data match that previously reported in the literature. ${ }^{11}$

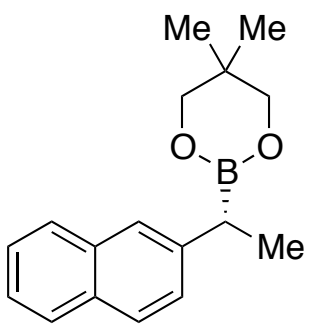


(R)-5,5-dimethyl-2-(1-(naphthalen-2-yl)ethyl)-1,3,2-dioxaborinane (2l). Prepared via General Procedure A using ammonium salt 1a (amine purchased in $>99 \%$ ee) and bis(neopentyl glycolato)diboron $\left(\mathrm{B}_{2}\right.$ neop 2 ) instead of $\mathrm{B}_{2} \mathrm{pin}_{2}$. Instead of filtering through Celite $^{\circledR}$, the diluted mixture was filtered through a small plug of silica gel and then concentrated. 1,3,5-Trimethoxybenzene was added as internal standard, and the yield of the reaction was determined by ${ }^{1} \mathrm{H}$ NMR analysis to be $61 \%$. The sample was then concentrated and subjected to General Procedure $\mathrm{C}$ for oxidation and determination of enantiomeric excess.

Boronate 2l was oxidized to alcohol 3a via General Procedure C. The enantiomeric excess was determined to be $95 \%$ by chiral HPLC analysis. See alcohol 3a below.<smiles>CC(O)c1ccc2ccccc2c1</smiles>

(R)-1-(naphthalen-2-yl)ethanol (3a). Prepared via General Procedure C using benzylic boronate 21. The crude mixture was purified by silica gel chromatography $(20 \%$ EtOAc/hexanes) to give 3a (29 mg, 93\%) as a white solid. The enantiomeric excess was determined to be $95 \%$ by chiral HPLC analysis (CHIRALPAK IA, $1.0 \mathrm{~mL} / \mathrm{min}, 1 \% i$ $\mathrm{PrOH} /$ hexanes, $\lambda=254 \mathrm{~nm}) ; \mathrm{t}_{\mathrm{R}}$ (major) $=45.06 \mathrm{~min}, \mathrm{t}_{\mathrm{R}}($ minor $)=47.29 \mathrm{~min}$. The spectral data match that of alcohol 3a above.

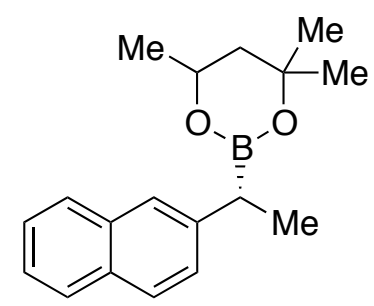

4,4,6-trimethyl-2-((R)-1-(naphthalen-2-yl)ethyl)-1,3,2-dioxaborinane (2m). Prepared via General Procedure A using ammonium salt 1a (amine purchased in $>99 \%$ ee) and bis(hexylene glycolato)diboron $\left(\mathrm{B}_{2} \mathrm{hex}_{2}\right)$ instead of $\mathrm{B}_{2} \mathrm{pin}_{2}$. Instead of filtering through 
Celite ${ }^{\circledR}$, the diluted mixture was filtered through a small plug of silica gel and then concentrated. 1,3,5-Trimethoxybenzene was added as internal standard, and the yield of the reaction was determined by ${ }^{1} \mathrm{H}$ NMR analysis to be $74 \%$. The sample was then concentrated and subjected to General Procedure $\mathrm{C}$ for oxidation and determination of enantiomeric excess.

Boronate 2m was oxidized to alcohol 3a via General Procedure C. The enantiomeric excess was determined to be $95 \%$ by chiral HPLC analysis. See alcohol 3a below.<smiles>CC(O)c1ccc2ccccc2c1</smiles>

(R)-1-(naphthalen-2-yl)ethanol (3a). Prepared via General Procedure C using benzylic boronate $\mathbf{2} \mathbf{m}$. The crude mixture was purified by silica gel chromatography $(20 \%$ EtOAc/hexanes) to give 3a (35 mg, quant.) as a white solid. The enantiomeric excess was determined to be $95 \%$ by chiral HPLC analysis (CHIRALPAK IA, $1.0 \mathrm{~mL} / \mathrm{min}, 1 \% i$ $\mathrm{PrOH} /$ hexanes, $\lambda=254 \mathrm{~nm}) ; \mathrm{t}_{\mathrm{R}}$ (major) $=44.64 \mathrm{~min}, \mathrm{t}_{\mathrm{R}}($ minor $)=46.84 \mathrm{~min}$. The spectral data match that of alcohol 3a above.

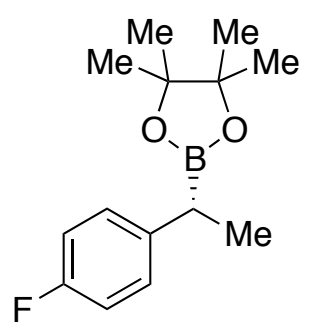

(R)-2-(1-(4-fluorophenyl)ethyl)-4,4,5,5-tetramethyl-1,3,2-dioxaborolane

(2n).

Prepared via General Procedure B on a 0.5 -mmol scale using ammonium salt 1n (amine purchased in $>99 \%$ ee $)$ and $\mathrm{ICy}^{-} \cdot \mathrm{HBF}_{4}(19.2 \mathrm{mg}, 0.060 \mathrm{mmol}, 12 \mathrm{~mol} \%)$ instead of $\mathrm{PPh}_{2} \mathrm{Cy}$. The crude mixture was purified by silica gel chromatography $(5 \%$ EtOAc/hexanes) to give $\mathbf{2 n}(66 \mathrm{mg}, 53 \%$ ) as a clear oil (please note that $\mathbf{2 n}$ was not 
subjected to high vacuum due to its volatility): ${ }^{1} \mathrm{H}$ NMR (400 MHz, $\left.\mathrm{CDCl}_{3}\right) \delta 7.20-7.13$ (m, 2H), $6.98-6.91(\mathrm{~m}, 2 \mathrm{H}), 2.42$ (q, $J=7.5 \mathrm{~Hz}, 1 \mathrm{H}), 1.31$ (d, $J=7.5 \mathrm{~Hz}, 3 \mathrm{H}), 1.21$ (s, $6 \mathrm{H}), 1.20(\mathrm{~s}, 6 \mathrm{H}) ;{ }^{13} \mathrm{C} \mathrm{NMR}\left(101 \mathrm{MHz}, \mathrm{CDCl}_{3}\right) \delta 161.0\left(\mathrm{~d}, J_{\mathrm{C}-\mathrm{F}}=243.2 \mathrm{~Hz}\right), 140.7\left(\mathrm{~d}, J_{\mathrm{C}-}\right.$ $\mathrm{F}=3.1 \mathrm{~Hz}), 129.1\left(\mathrm{~d}, J_{\mathrm{C}-\mathrm{F}}=7.7 \mathrm{~Hz}\right), 115.1\left(\mathrm{~d}, J_{\mathrm{C}-\mathrm{F}}=21.0 \mathrm{~Hz}\right), 83.5,24.8$, 17.4. The spectral data match that reported in the literature. ${ }^{12}$ The sample was then concentrated and subjected to General Procedure $\mathrm{C}$ for oxidation and determination of enantiomeric excess.

Boronate 2n was oxidized to alcohol 3n via General Procedure C. The enantiomeric excess was determined to be $86 \%$ (run 1 from oxidation of isolated $2 \mathrm{n}$ : $87 \%$ ee; run 2 from oxidation of crude $\mathbf{2 n}$ : 85\% ee) by chiral HPLC analysis. See alcohol $\mathbf{3 n}$ below.

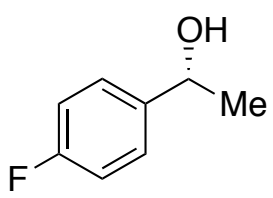

(R)-1-(4-fluorophenyl)ethanol (3n). Prepared via General Procedure C using benzylic boronate 2 n. The crude mixture was purified by silica gel chromatography $(20 \%$ EtOAc/hexanes) to give $3 \mathbf{n}(16 \mathrm{mg}, 62 \%)$ as a clear oil. The enantiomeric excess was determined to be $86 \%$ (run 1 from oxidation of isolated $2 \mathrm{n}$ : $87 \%$ ee; run 2 from oxidation of crude $2 \mathrm{n}$ : $85 \%$ ee) by chiral HPLC analysis (CHIRALPAK IF, $1.0 \mathrm{~mL} / \mathrm{min}, 2 \% \mathrm{i}$ $\mathrm{PrOH} /$ hexanes, $\lambda=254 \mathrm{~nm})$; $\mathrm{t}_{\mathrm{R}}$ (major) $=16.25 \mathrm{~min}, \mathrm{t}_{\mathrm{R}}$ (minor) $=17.65 \mathrm{~min}:{ }^{1} \mathrm{H}$ NMR $(600$ $\left.\mathrm{MHz}, \mathrm{CDCl}_{3}\right) \delta 7.34(\mathrm{dd}, J=8.3,5.6 \mathrm{~Hz}, 2 \mathrm{H}), 7.03(\mathrm{t}, J=8.6 \mathrm{~Hz}, 2 \mathrm{H}), 4.89(\mathrm{q}, J=6.4$ $\mathrm{Hz}, 1 \mathrm{H}), 1.87(\mathrm{~s}, 1 \mathrm{H}), 1.48(\mathrm{~d}, J=6.5 \mathrm{~Hz}, 3 \mathrm{H}) ;{ }^{13} \mathrm{C} \mathrm{NMR}\left(151 \mathrm{MHz}, \mathrm{CDCl}_{3}\right) \delta 162.3(\mathrm{~d}$, $\left.J_{\mathrm{C}-\mathrm{F}}=244.6 \mathrm{~Hz}\right), 141.7,127.2\left(\mathrm{~d}, J_{\mathrm{C}-\mathrm{F}}=7.6 \mathrm{~Hz}\right), 115.4\left(\mathrm{~d}, J_{\mathrm{C}-\mathrm{F}}=22.7 \mathrm{~Hz}\right), 70.0,25.5$; ${ }^{19} \mathrm{~F} \mathrm{NMR}\left(565 \mathrm{MHz}, \mathrm{CDCl}_{3}\right) \delta-115.4$. The spectral data match that of the literature. ${ }^{13}$ 


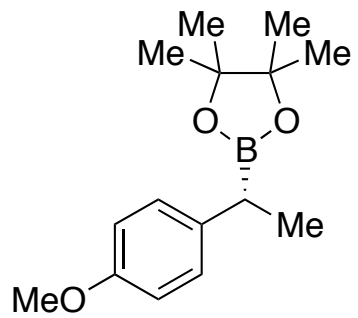

(R)-2-(1-(4-methoxyphenyl)ethyl)-4,4,5,5-tetramethyl-1,3,2-dioxaborolane

(20).

Prepared via General Procedure B using ammonium salt 10 (amine precursor purchased in $>99 \%$ ee). The crude mixture was purified by silica gel chromatography (5\% EtOAc/hexanes) to give 20 (run 1: $45 \mathrm{mg}, 57 \%$; run 2: $41 \mathrm{mg}, 52 \%$ ) as a clear oil: ${ }^{1} \mathrm{H}$ NMR (400 MHz, $\left.\mathrm{CDCl}_{3}\right) \delta 7.14(\mathrm{~d}, J=8.6 \mathrm{~Hz}, 2 \mathrm{H}), 6.82(\mathrm{~d}, J=8.6 \mathrm{~Hz}, 2 \mathrm{H}), 3.78(\mathrm{~s}$, $3 \mathrm{H}), 2.38$ (q, $J=7.5 \mathrm{~Hz}, 1 \mathrm{H}), 1.30(\mathrm{~d}, J=7.6 \mathrm{~Hz}, 3 \mathrm{H}), 1.22(\mathrm{~s}, 6 \mathrm{H}), 1.20(\mathrm{~s}, 6 \mathrm{H}) ;{ }^{13} \mathrm{C}$ NMR $\left(101 \mathrm{MHz}, \mathrm{CDCl}_{3}\right) \delta 157.2,137.0,128.6,113.8,83.2,55.2,24.7,24.6,17.4 .^{4}$ The spectral data matches that previously reported in the literature. ${ }^{14}$

Boronate 20 was oxidized to alcohol 30 via General Procedure C. The enantiomeric excess was determined to be $86 \%$ (run 1: $85 \%$ ee; run $2: 87 \%$ ee) by chiral HPLC analysis. See alcohol 30 below.

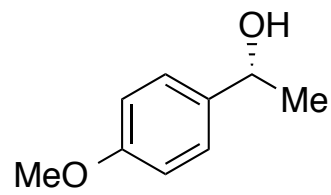

(R)-1-(4-methoxyphenyl)ethanol (3o). Prepared via General Procedure C using benzylic boronate 2o. The crude mixture was purified by silica gel chromatography $(20 \%$ EtOAc/hexanes) to give 3o (run 1 (45 $\mathrm{mg}$ of 2o): $19 \mathrm{mg}, 72 \%$ ) as a clear oil. The enantiomeric excess was determined to be $86 \%$ (run 1: $85 \%$, run $2: 87 \%$ ) by chiral HPLC analysis (CHIRALPAK IB, $1.0 \mathrm{~mL} / \mathrm{min}, 2 \% i$-PrOH/hexanes, $\lambda=254 \mathrm{~nm}$ ); $t_{\mathrm{R}}$ (major) $=$ $20.16 \mathrm{~min}, \mathrm{t}_{\mathrm{R}}$ (minor) $=22.24 \mathrm{~min}:{ }^{1} \mathrm{H} \mathrm{NMR}\left(400 \mathrm{MHz}, \mathrm{CDCl}_{3}\right) \delta 7.30(\mathrm{~d}, J=8.6 \mathrm{~Hz}$, 2H), $6.88(\mathrm{~d}, J=8.6 \mathrm{~Hz}, 2 \mathrm{H}), 4.86(\mathrm{q}, J=6.4 \mathrm{~Hz}, 1 \mathrm{H}), 3.80(\mathrm{~s}, 3 \mathrm{H}), 1.84(\mathrm{bs}, 1 \mathrm{H}), 1.48$ $(\mathrm{d}, J=6.4 \mathrm{~Hz}, 3 \mathrm{H}) ;{ }^{13} \mathrm{C}$ NMR $\left(101 \mathrm{MHz}, \mathrm{CDCl}_{3}\right) \delta 159.1,138.1,126.8,114.0,70.2$, $55.5,25.2$. The spectral data match that previously reported in the literature. ${ }^{15}$ 


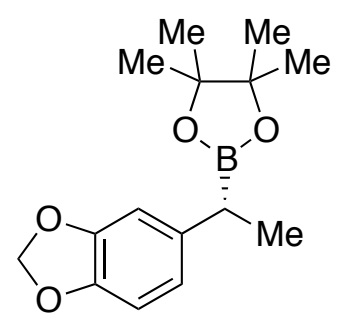

\section{(R)-2-(1-(benzo[d][1,3]dioxol-5-yl)ethyl)-4,4,5,5-tetramethyl-1,3,2-dioxaborolane}

(2p). Prepared via General Procedure B using ammonium salt 1p (amine prepared in $\geq 95 \%$ ee). The crude mixture was purified by silica gel chromatography (5\% EtOAc/hexanes) to give 2p (run 1: $48 \mathrm{mg}, 58 \%$; run 2: $53 \mathrm{mg}, 63 \%$ ) as a clear oil: ${ }^{1} \mathrm{H}$ NMR (400 MHz, CDCl $) \delta 6.74(\mathrm{~d}, J=1.7 \mathrm{~Hz}, 1 \mathrm{H}), 6.71(\mathrm{~d}, J=8.0 \mathrm{~Hz}, 1 \mathrm{H}), 6.65(\mathrm{dd}, J$ $=8.0,1.7 \mathrm{~Hz}, 1 \mathrm{H}), 5.90(\mathrm{~s}, 2 \mathrm{H}), 2.35(\mathrm{q}, J=7.5 \mathrm{~Hz}, 1 \mathrm{H}), 1.28(\mathrm{~d}, J=7.5 \mathrm{~Hz}, 3 \mathrm{H}), 1.22$ (s, 6H), $1.20(\mathrm{~s}, 6 \mathrm{H}) ;{ }^{13} \mathrm{C} \mathrm{NMR}\left(101 \mathrm{MHz}, \mathrm{CDCl}_{3}\right) \delta 147.6,145.2,139.0,120.5,108.6$, 108.3, 100.8, 83.5, 24.8, 24.8, 17.7; ${ }^{411} \mathrm{~B}$ NMR (193 MHz, $\left.\mathrm{CDCl}_{3}\right) \delta 33.3$; FTIR (neat) 2977, 1487, 1321, 1237, 1144, 1041, 938, $811 \mathrm{~cm}^{-1}$; HRMS (CI) calculated for $\mathrm{C}_{15} \mathrm{H}_{21} \mathrm{BO}_{4}:$ 277.1611, found: 277.1609.

Boronate $\mathbf{2 p}$ was oxidized to alcohol $\mathbf{3 p}$ via General Procedure C. The enantiomeric excess was determined to be $85 \%$ (run 1: $84 \%$ ee; run 2: $85 \%$ ee) by chiral HPLC analysis. See alcohol 3p below.

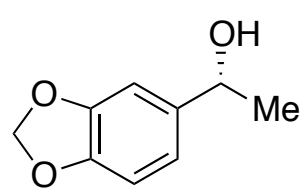

(R)-1-(benzo $[d][1,3]$ dioxol-5-yl)ethanol (3p). Prepared via General Procedure C using benzylic boronate $\mathbf{2 p}$. The crude mixture was purified by silica gel chromatography $(20 \%$ EtOAc/hexanes) to give 3p (run 1 (40 $\mathrm{mg}$ of 2o): $21 \mathrm{mg}, 87 \%$ ) as a clear oil. The enantiomeric excess was determined to be $85 \%$ (run $1: 85 \%$, run $2: 84 \%$ ) by chiral HPLC analysis (CHIRALPAK IA, $1.0 \mathrm{~mL} / \mathrm{min}, 3 \% i-\mathrm{PrOH} /$ hexanes, $\lambda=210 \mathrm{~nm}$ ); $\mathrm{t}_{\mathrm{R}}$ (major) $=$ $19.60 \mathrm{~min}, \mathrm{t}_{\mathrm{R}}$ (minor) $=22.01 \mathrm{~min}:{ }^{1} \mathrm{H}$ NMR $\left(400 \mathrm{MHz}, \mathrm{CDCl}_{3}\right) \delta 6.92-6.88(\mathrm{~m}, 1 \mathrm{H})$, $6.85-6.75$ (m, 2H), 5.95 (s, 2H), 4.82 (q, $J=6.4 \mathrm{~Hz}, 1 \mathrm{H}), 1.76$ (bs, 1H), 1.46 (d, $J=6.4$ $\mathrm{Hz}, 3 \mathrm{H}) ;{ }^{13} \mathrm{C} \mathrm{NMR}\left(101 \mathrm{MHz}, \mathrm{CDCl}_{3}\right) \delta 147.9,147.0,140.1,118.9,108.3,106.2,101.2$, 70.5, 25.3. The spectral data match that previously reported in the literature. ${ }^{15}$ 


\section{Gram-Scale Synthesis of (S)-2-(1-(6-methoxynaphthalen-2- yl)ethyl)-4,4,5,5-tetramethyl-1,3,2-dioxaborolane (2c)}

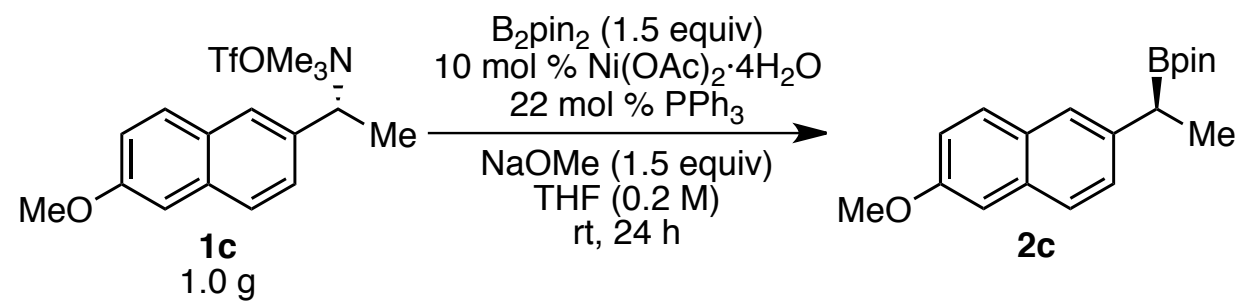

An oven-dried, 50-mL Schlenk flask equipped with a magnetic stir bar was evacuated and backfilled with $\mathrm{N}_{2}$ (x 4). $\mathrm{Ni}(\mathrm{OAc})_{2} \cdot 4 \mathrm{H}_{2} \mathrm{O}(63 \mathrm{mg}, 0.254 \mathrm{mmol}, 10 \mathrm{~mol} \%), \mathrm{PPh}_{3}(147$ $\mathrm{mg}, 0.559 \mathrm{mmol}, 22 \mathrm{~mol} \%$ ), $\mathrm{B}_{2} \operatorname{pin}_{2}(0.968 \mathrm{~g}, 3.81 \mathrm{mmol}, 1.5$ equiv), and ammonium salt 1 c ( $\geq 95 \%$ ee, $1.00 \mathrm{~g}, 2.54$ mmol. 1.0 equiv) were added. NaOMe $(0.206 \mathrm{~g}, 3.81 \mathrm{mmol}$, 1.5 equiv) was quickly added and the flask was sealed with a rubber septum. The flask was evacuated and then backfilled with $\mathrm{N}_{2}$ (x 4). THF (13 mL, $0.2 \mathrm{M}$ ) was then added. The mixture was stirred vigorously at room temperature for $24 \mathrm{~h}$. Over the course of the reaction, the solution turned from light yellow to dark orange. $\mathrm{Et}_{2} \mathrm{O}(\sim 40 \mathrm{~mL})$ was added, and the mixture was stirred for five minutes. The mixture was filtered through a pad of Celite ${ }^{\circledR}$, which was then washed multiple times with $\mathrm{Et}_{2} \mathrm{O}(\sim 120 \mathrm{~mL}$ total volume). The filtrate was concentrated, and the crude material was purified by silica gel chromatography (5\% EtOAc/hexanes) to give $2 \mathrm{c}(68 \%, 99 \%$ ee) as a white solid. The spectra of this material match that of $2 \mathbf{c}$ prepared on $0.3 \mathrm{mmol}$ scale, as described above.

Boronate 2c was oxidized to alcohol 3c via General Procedure C. The enantiomeric excess was determined to be $99 \%$ by chiral HPLC analysis. The spectral data of this alcohol match that of alcohol $\mathbf{2 c}$ as described above.

\section{Preparation of Benzyl Ammonium Salts}

Enantioenriched amines that were not commercially available were obtained through Grignard or hydride additions to Ellman's sulfinimines. ${ }^{2}$ Via these reactions, a single diastereomer of each sulfinamine was isolated (as determined by ${ }^{1} \mathrm{H}$ NMR analysis). We thus assume $\geq 95 \%$ ee of the subsequent amine after removal of Ellman's 
auxiliary. Dimethyl benzyl amines were then prepared using Escheweiler-Clarke conditions or reductive amination of the corresponding primary benzyl amine with formaldehyde. ${ }^{3}$ We assume no loss of ee in the formation of the trimethyl ammonium triflates from this intermediate. For enantioenriched amines that were commercially available, we also assume no loss of ee in the formation of the trimethyl ammonium triflates.

Ammonium triflates $1 \mathbf{a}, \mathbf{1 b}, \mathbf{1 k}, \mathbf{1}$, and $1 \mathrm{o}$ have been previously prepared in our laboratory. ${ }^{16}$

Ammonium triflates prepared via these procedures were used as is in the stereospecific borylation reaction, without further purification. In some cases, impurities are present in the ammonium triflates.

\section{General Procedure D: Preparation of (S)-N,N,N-trimethyl-1-(naphthalen-1- yl)ethanaminium trifluoromethanesulfonate (1b)}
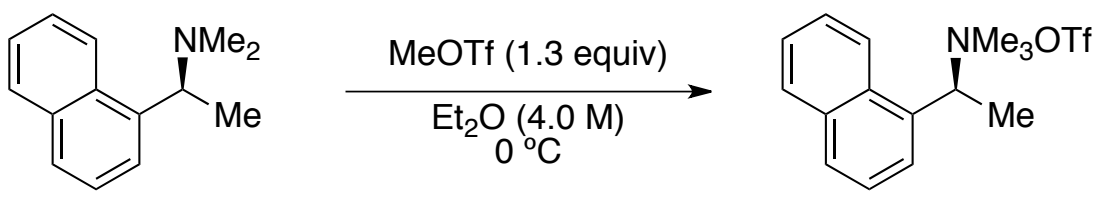

(S)- $N, N$-Dimethyl-1-(naphthalen-1-yl)ethanamine (0.806 g, $4.04 \mathrm{mmol}, 1.0$ equiv), which was prepared using Escheweiler-Clarke conditions $^{3 \mathrm{a}}$ from (S)-(-)-1-(1naphthyl)ethylamine (purchased in $>99 \%$ ee), was dissolved in $\mathrm{Et}_{2} \mathrm{O}(1.01 \mathrm{~mL}, 4.0 \mathrm{M})$. MeOTf $\left(0.58 \mathrm{~mL}, 5.25 \mathrm{mmol}, 1.3\right.$ equiv) was added dropwise at $0{ }^{\circ} \mathrm{C}$. After complete addition, the mixture was allowed to stir for an additional 30 minutes at $0{ }^{\circ} \mathrm{C}$. The mixture was diluted with $\mathrm{Et}_{2} \mathrm{O}(\sim 2 \mathrm{~mL})$, taken out of the ice bath, and allowed to warm to room temperature while stirring. The white precipitate was filtered and washed with $\mathrm{Et}_{2} \mathrm{O}(3 \times 15 \mathrm{~mL})$. The solid was dried under high vacuum to afford salt $\mathbf{1 b}(1.377 \mathrm{~g}$, 
94\%) as a white solid, which was used directly in the benzylic borylation. This compound was previously prepared in our laboratory via this method. ${ }^{15}$<smiles>COc1ccc2cc(C(C)C)ccc2c1</smiles>

\section{(R)-1-(6-methoxynaphthalen-2-yl)- $N, N, N$-trimethylethanaminium}

trifluoromethanesulfonate (1c). Prepared according to General Procedure D on a 5.64 mmol scale from $(R)$-1-(6-methoxynaphthalen-2-yl)- $N, N$-dimethylethanamine, which was

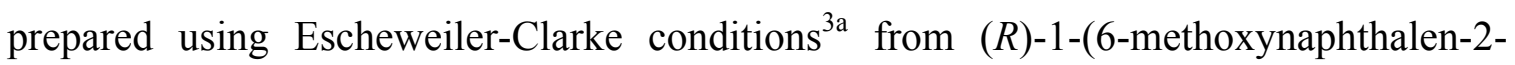
yl)ethanamine (prepared using Ellman's auxiliary ${ }^{2}$ ), to afford salt 1c (2.085 g, 94\%) as a white solid (mp 109-111 $\left.{ }^{\circ} \mathrm{C}\right):{ }^{1} \mathrm{H}$ NMR $\left(600 \mathrm{MHz}, \mathrm{CDCl}_{3}\right) \delta 7.94$ (s, 1H), 7.84-7.74 (m, 2H), 7.49 (d, $J=8.3 \mathrm{~Hz}, 1 \mathrm{H}), 7.19$ (dd, $J=9.0,2.4 \mathrm{~Hz}, 1 \mathrm{H}), 7.13$ (d, $J=2.1 \mathrm{~Hz}, 1 \mathrm{H})$, $4.98(\mathrm{q}, J=6.9 \mathrm{~Hz}, 1 \mathrm{H}), 3.92(\mathrm{~s}, 3 \mathrm{H}), 3.15(\mathrm{~s}, 9 \mathrm{H}), 1.88(\mathrm{~d}, J=6.8 \mathrm{~Hz}, 3 \mathrm{H}) ;{ }^{13} \mathrm{C} \mathrm{NMR}$ $\left(151 \mathrm{MHz}, \mathrm{CDCl}_{3}\right) \delta 159.3,135.6,130.2,128.4,128.1,127.2,121.0\left(\mathrm{q}, J_{\mathrm{C}-\mathrm{F}}=320.1 \mathrm{~Hz}\right)$, 120.4, 105.7, 74.5, 55.6, 51.2, 15.2; ${ }^{19} \mathrm{~F}$ NMR (376.5 MHz, $\left.\mathrm{CDCl}_{3}\right) \delta-78.4$; FTIR (neat) 3043, 1608, 1488, 1270, 1160, 846, $639 \mathrm{~cm}^{-1}$; LRMS (ESI+) $[\mathrm{M-OTf}]^{+}$calculated for $\left[\mathrm{C}_{16} \mathrm{H}_{22} \mathrm{NO}^{+}\right.$]: 244.2, found: 244.2.

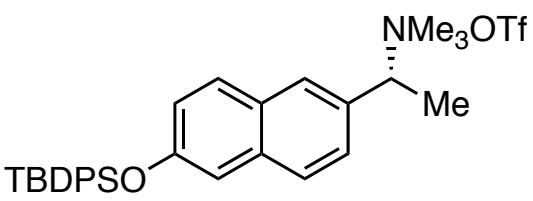

\section{(R)-1-(6-((tert-butyldiphenylsilyl)oxy)naphthalen-2-yl)- $N, N, N-$}

trimethylethanaminium trifluoromethanesulfonate (1d). Prepared according to General Procedure D on a $1.50 \mathrm{mmol}$ scale from (R)-1-(6-((tertbutyldiphenylsilyl)oxy)naphthalen-2-yl)- $N, N$-dimethylethanamine, which was prepared by reductive amination $^{3 \mathrm{~b}}$ from $(R)-1-(6-(($ tert-butyldiphenylsilyl)oxy)naphthalen-2yl)ethanamine (prepared using Ellman's auxiliary ${ }^{2}$ ). In this case, stirring ceased as a 
result of precipitate formation. The solution was diluted with $\mathrm{Et}_{2} \mathrm{O}$ to $2.0 \mathrm{M}$ and the stir bar was agitated with a spatula to resume stirring. The reaction afforded salt $\mathbf{1 d}(0.698 \mathrm{~g}$, $75 \%$ ) as a white solid (mp 180-182 $\left.{ }^{\circ} \mathrm{C}\right):{ }^{1} \mathrm{H}$ NMR $\left(600 \mathrm{MHz}, \mathrm{CDCl}_{3}\right) \delta 7.87(\mathrm{~s}, 1 \mathrm{H}), 7.74$ (d, $J=6.9 \mathrm{~Hz}, 4 \mathrm{H}), 7.69$ (d, $J=8.9 \mathrm{~Hz}, 1 \mathrm{H}), 7.53$ (d, $J=8.6 \mathrm{~Hz}, 1 \mathrm{H}), 7.45-7.40$ (m, 2H), $7.39-7.33(\mathrm{~m}, 5 \mathrm{H}), 7.13(\mathrm{dd}, J=8.9,2.4 \mathrm{~Hz}, 1 \mathrm{H}), 7.05(\mathrm{~d}, J=2.1 \mathrm{~Hz}, 1 \mathrm{H}), 4.92$ (q, $J=6.9 \mathrm{~Hz}, 1 \mathrm{H}), 3.13$ (s, 9H), 1.84 (d, $J=6.9 \mathrm{~Hz}, 3 \mathrm{H}), 1.13$ (s, 9H); ${ }^{13} \mathrm{C}$ NMR $(151 \mathrm{MHz}$, $\left.\mathrm{CDCl}_{3}\right) \delta 155.3,135.6,135.4,132.6,130.3,130.1,128.5,128.14,128.09,127.2,123.3$, $120.9\left(\mathrm{q}, J_{\mathrm{C}-\mathrm{F}}=320.1 \mathrm{~Hz}\right), 114.6,74.6,51.2,26.7,19.7,15.2 ;{ }^{19} \mathrm{~F}$ NMR $(565 \mathrm{MHz}$, $\left.\mathrm{CDCl}_{3}\right) \delta-78.4 ;{ }^{29} \mathrm{Si} \mathrm{NMR}\left(119 \mathrm{MHz}, \mathrm{CDCl}_{3}\right) \delta-5.0$; FTIR (neat) 3051, 2933, 2859, $1605,1483,1266,1161,1031,879,703,639 \mathrm{~cm}^{-1}$; LRMS (ESI+) $[\mathrm{M}-\mathrm{OTf}]^{+}$calculated for $\left[\mathrm{C}_{31} \mathrm{H}_{38} \mathrm{NOSi}^{+}\right]$: 468.3 , found: 468.4 .

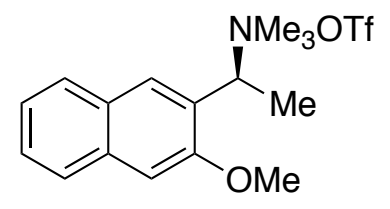

\section{(S)-1-(3-methoxynaphthalen-2-yl)- $N, N, N$-trimethylethanaminium}

trifluoromethanesulfonate (1e) Prepared according to General Procedure D on a 1.45 mmol scale from (S)-1-(3-methoxynaphthalen-2-yl)- $N, N$-dimethylethanamine, which was prepared using Escheweiler-Clarke conditions ${ }^{3 a}$ from (S)-1-(3-methoxynaphthalen-2yl)ethanamine (prepared using Ellman's auxiliary ${ }^{2}$ ). In this case, a white precipitate did not form upon addition of MeOTf. Instead, two distinct layers formed. The top layer was decanted off. The bottom layer was washed with $\mathrm{Et}_{2} \mathrm{O}(5 \times 2 \mathrm{~mL})$ and then hexanes $(5 \times 2$ $\mathrm{mL}$, HPLC grade) and then dried under high vacuum to give salt $1 \mathrm{e}(0.359 \mathrm{~g}, 63 \%)$ as a clear viscous oil. By NMR, an $\sim 8: 1$ mixture of rotamers was observed: ${ }^{1} \mathrm{H}$ NMR (600 $\mathrm{MHz}, \mathrm{CDCl}_{3}$, major rotamer) $\delta 7.97(\mathrm{~s}, 1 \mathrm{H}), 7.87(\mathrm{~d}, J=8.2 \mathrm{~Hz}, 1 \mathrm{H}), 7.73(\mathrm{~d}, J=8.3 \mathrm{~Hz}$, $1 \mathrm{H}), 7.50(\mathrm{t}, J=7.6 \mathrm{~Hz}, 1 \mathrm{H}), 7.38(\mathrm{t}, J=7.5 \mathrm{~Hz}, 1 \mathrm{H}), 7.22(\mathrm{~s}, 1 \mathrm{H}), 5.29(\mathrm{q}, J=7.1 \mathrm{~Hz}$, $1 \mathrm{H}), 3.98(\mathrm{~s}, 3 \mathrm{H}), 3.11(\mathrm{~s}, 9 \mathrm{H}), 1.85(\mathrm{~d}, J=7.0 \mathrm{~Hz}, 3 \mathrm{H}) ;{ }^{13} \mathrm{C} \mathrm{NMR}\left(151 \mathrm{MHz}, \mathrm{CDCl}_{3}\right.$, major rotamer) $\delta 154.8,135.3,130.7,128.7,128.5,128.1,126.6,125.1,122.5,120.7$ (q, $\left.J_{\mathrm{C}-\mathrm{F}}=320.0 \mathrm{~Hz}\right), 106.8,65.9,56.0,51.09,51.07,51.05,15.4 ;{ }^{17}{ }^{19} \mathrm{~F}$ NMR $(376.5 \mathrm{MHz}$, 
$\left.\mathrm{CDCl}_{3}\right) \delta$-78.4; FTIR (neat) 3048, 1634, 1474, 1260, 1163, 1031, 756, $639 \mathrm{~cm}^{-1}$; LRMS $(\mathrm{ESI}+)[\mathrm{M}-\mathrm{OTf}]^{+}$calculated for $\left[\mathrm{C}_{16} \mathrm{H}_{22} \mathrm{NO}^{+}\right]$: 244.2, found: 244.2 .

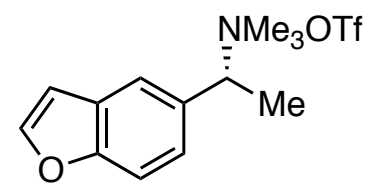

(R)-1-(benzofuran-5-yl)- $N, N, N$-trimethylethanaminium trifluoromethanesulfonate (1f). Prepared according to General Procedure D on a 6.12 mmol scale from $(R)-1$ (benzofuran-5-yl)- $N, N$-dimethylethanamine, which was prepared using EscheweilerClarke conditions $^{3 \mathrm{a}}$ from $(R)$-1-(benzofuran-5-yl)ethanamine (prepared using Ellman's auxiliary $^{2}$ ). In this case, a white precipitate did not form upon addition of MeOTf. Instead, two distinct layers formed. The top layer was decanted off. The bottom layer was washed with $\mathrm{Et}_{2} \mathrm{O}(2 \mathrm{~mL})$, which caused white precipitate to form. The precipitate was filtered and washed with $\mathrm{Et}_{2} \mathrm{O}(3 \times 15 \mathrm{~mL})$ and dried under high vacuum to afford salt $\mathbf{1 f}$ $(2.076 \mathrm{~g}, 96 \%)$ as a white solid (mp 106-108 $\left.{ }^{\circ} \mathrm{C}\right):{ }^{1} \mathrm{H}$ NMR $\left(600 \mathrm{MHz}, \mathrm{CDCl}_{3}\right) \delta 7.81$ (s, $1 \mathrm{H}), 7.68(\mathrm{~d}, J=2.1 \mathrm{~Hz}, 1 \mathrm{H}), 7.55(\mathrm{~d}, J=8.5 \mathrm{~Hz}, 1 \mathrm{H}), 7.41(\mathrm{~d}, J=8.2 \mathrm{~Hz}, 1 \mathrm{H}), 6.85-$ $6.81(\mathrm{~m}, 1 \mathrm{H}), 4.98$ (q, $J=7.0 \mathrm{~Hz}, 1 \mathrm{H}), 3.13(\mathrm{~s}, 9 \mathrm{H}), 1.85$ (d, $J=6.9 \mathrm{~Hz}, 3 \mathrm{H}) ;{ }^{13} \mathrm{C}$ NMR $\left(151 \mathrm{MHz}, \mathrm{CDCl}_{3}\right) \delta 155.8,146.9,128.5,127.1,120.9\left(\mathrm{q}, J_{\mathrm{C}-\mathrm{F}}=320.1 \mathrm{~Hz}\right), 112.4,107.0$, 74.4, 51.18, 51.15, 51.1, 15.5; ${ }^{17}{ }^{19} \mathrm{~F}$ NMR (565 MHz, $\left.\mathrm{CDCl}_{3}\right) \delta$-78.4; FTIR (neat) 3042, $1472,1263,1158,1030,838,750,639,518 \mathrm{~cm}^{-1}$; LRMS (ESI+) [M-OTf] ${ }^{+}$calculated for $\left[\mathrm{C}_{13} \mathrm{H}_{18} \mathrm{NO}^{+}\right]$: 204.1, found: 204.2.

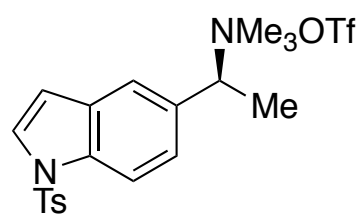

( $S$ )- $N, N, N$-trimethyl-1-(1-tosyl-1 $H$-indol-5-yl)ethanaminium

trifluoromethanesulfonate (1g). Prepared according to General Procedure D on a 2.97 mmol scale from ( $S$ )- $N, N$-dimethyl-1-(1-tosyl-1 $H$-indol-5-yl)ethanamine, which was 
prepared using Escheweiler-Clarke conditions ${ }^{3 a}$ from $(S)$-1-(1-tosyl-1 $H$-indol-5-

yl)ethanamine (prepared using Ellman's auxiliary $\left.{ }^{2}\right)$ to afford salt $1 \mathbf{g}(1.277 \mathrm{~g}, 85 \%)$ as a white solid (mp 73-75 $\left.{ }^{\circ} \mathrm{C}\right) ;{ }^{1} \mathrm{H}$ NMR $\left(600 \mathrm{MHz}, \mathrm{CDCl}_{3}\right) \delta 8.01(\mathrm{~d}, J=8.6 \mathrm{~Hz}, 1 \mathrm{H}), 7.79-$ $7.73(\mathrm{~m}, 3 \mathrm{H}), 7.62(\mathrm{~d}, J=3.7 \mathrm{~Hz}, 1 \mathrm{H}), 7.42(\mathrm{~d}, J=8.6 \mathrm{~Hz}, 1 \mathrm{H}), 7.25(\mathrm{~s}, 1 \mathrm{H}), 6.71(\mathrm{~d}, J=$ $3.6 \mathrm{~Hz}, 1 \mathrm{H}), 4.91$ (q, $J=6.9 \mathrm{~Hz}, 1 \mathrm{H}), 3.09$ (s, 9H), 2.34 (s, 3H), 1.80 (d, $J=6.9 \mathrm{~Hz}, 3 \mathrm{H}$ ); ${ }^{13} \mathrm{C}$ NMR $\left(151 \mathrm{MHz}, \mathrm{CDCl}_{3}\right) \delta 145.8,135.6,135.0,131.3,130.4,128.0,127.4,127.1$, $120.9\left(\mathrm{q}, J_{\mathrm{C}-\mathrm{F}}=320.12 \mathrm{~Hz}\right), 114.2,109.1,74.3,51.2,21.8,15.4 ;{ }^{19} \mathrm{~F}$ NMR $(565 \mathrm{MHz}$, $\left.\mathrm{CDCl}_{3}\right) \delta$-78.4; FTIR (neat) 3051, 1464, 1373, 1273, 1175, 1031, 639, $581 \mathrm{~cm}^{-1}$; LRMS $(\mathrm{ESI}+)$ [M-OTf] $]^{+}$calculated for $\left[\mathrm{C}_{20} \mathrm{H}_{25} \mathrm{~N}_{2} \mathrm{O}_{2} \mathrm{~S}^{+}\right]$: 357.2, found: 357.3 .

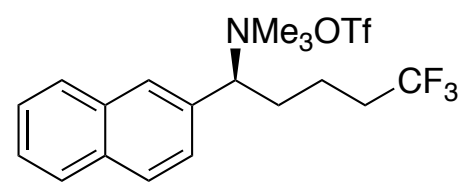

\section{(S)-5,5,5-trifluoro- $N, N, N$-trimethyl-1-(naphthalen-2-yl)pentan-1-aminium}

trifluoromethanesulfonate (1h). Prepared according to General Procedure D on a 3.47 mmol scale from (S)-5,5,5-trifluoro- $N, N$-dimethyl-1-(naphthalen-2-yl)pentan-1-amine, which was prepared using Escheweiler-Clarke conditions ${ }^{3 a}$ from $(S)-5,5,5$-trifluoro-1(naphthalen-2-yl)pentan-1-amine (prepared using Ellman's auxiliary ${ }^{2}$ ). In this case, a precipitate did not form upon addition of MeOTf. Instead, two distinct layers were observed. The top layer was decanted. The bottom layer was washed with a 1:1 (v/v) solution of $\mathrm{Et}_{2} \mathrm{O} /$ hexanes $(5 \times 4 \mathrm{~mL})$ and dried under high vacuum at $50{ }^{\circ} \mathrm{C}$ to afford salt 1h $(1.492 \mathrm{~g}, 94 \%)$ as a sticky solid: ${ }^{1} \mathrm{H}$ NMR $\left(600 \mathrm{MHz}, \mathrm{CDCl}_{3}\right) \delta 8.15-7.88(\mathrm{~m}, 3 \mathrm{H})$, $7.86(\mathrm{~d}, J=8.2 \mathrm{~Hz}, 1 \mathrm{H}), 7.64-7.44(\mathrm{~m}, 3 \mathrm{H}), 4.87-4.76(\mathrm{~m}, 1 \mathrm{H}), 3.16(\mathrm{~s}, 9 \mathrm{H}), 2.41$ (s, $2 \mathrm{H}), 2.29-2.03(\mathrm{~m}, 2 \mathrm{H}), 1.53-1.36(\mathrm{~m}, 1 \mathrm{H}), 1.32-1.13(\mathrm{~m}, 1 \mathrm{H}) ;{ }^{13} \mathrm{C} \mathrm{NMR}(101 \mathrm{MHz}$, $\left.\mathrm{CDCl}_{3}\right) \delta 135.0,134.1,133.1,130.0,129.6,128.7,128.2,127.9,127.5,126.9$ (q, $J_{\mathrm{C}-\mathrm{F}}=$ $277.5 \mathrm{~Hz}), 122.9,120.7\left(\mathrm{q}, J_{\mathrm{C}-\mathrm{F}}=320.1 \mathrm{~Hz}\right), 78.7,51.8,32.8\left(\mathrm{q}, J_{\mathrm{C}-\mathrm{F}}=29.0 \mathrm{~Hz}\right) \quad 26.3$, 19.2; ${ }^{19} \mathrm{~F}$ NMR (565 MHz, $\left.\mathrm{CDCl}_{3}\right) \delta-78.4,-66.2$; FTIR (neat) 3053, 2957, 1491, 1260, $1154,1031,831,639 \mathrm{~cm}^{-1}$; LRMS (ESI+) $[\mathrm{M}-\mathrm{OTf}]^{+}$calculated for $\left[\mathrm{C}_{18} \mathrm{H}_{23} \mathrm{~F}_{3} \mathrm{~N}^{+}\right]: 310.2$, found: 310.4. Two-dimensonal NMR experiments were used to verify ${ }^{1} \mathrm{H}$ and ${ }^{13} \mathrm{C}$ assignments due to the complex nature of the spectra. 


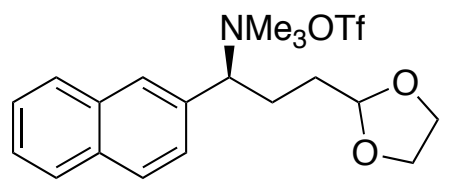

\section{(S)-3-(1,3-dioxolan-2-yl)- $N, N, N$-trimethyl-1-(naphthalen-2-yl)propan-1-aminium}

trifluoromethanesulfonate (1i). Prepared according to General Procedure D on a 1.93 mmol scale from (S)-3-(1,3-dioxolan-2-yl)- $N, N$-dimethyl-1-(naphthalen-2-yl)propan-1amine, which was prepared by reductive amination ${ }^{3 b}$ from (S)-3-(1,3-dioxolan-2-yl)-1(naphthalen-2-yl)propan-1-amine (prepared using Ellman's auxiliary ${ }^{2}$ ). In this case, a white precipitate did not form upon addition of MeOTf. Instead, two distinct layers formed. The top layer was decanted off. The bottom layer was washed with $\mathrm{Et}_{2} \mathrm{O}(5 \times 2$ $\mathrm{mL}$ ) and then hexanes ( $5 \times 2 \mathrm{~mL}$, HPLC grade) and then dried under high vacuum to give salt $1 \mathbf{i}(0.854 \mathrm{~g}, 98 \%)$ as a sticky white solid: ${ }^{1} \mathrm{H}$ NMR $\left(600 \mathrm{MHz}, \mathrm{CDCl}_{3}\right) \delta 8.15-7.89$ (m, 3H), $7.86(\mathrm{~d}, J=7.9 \mathrm{~Hz}, 1 \mathrm{H}), 7.63-7.41$ (m, 3H), $4.89-4.83$ (m, 1H), 4.81 (t, $J=$ $4.0 \mathrm{~Hz}, 1 \mathrm{H}), 3.97-3.86(\mathrm{~m}, 2 \mathrm{H}), 3.82-3.63(\mathrm{~m}, 2 \mathrm{H}$. Please note: this peak is contaminated with an unknown impurity. At $50{ }^{\circ} \mathrm{C}$ the peak corresponding to the impurity shifts and an accurate integration of two protons is obtained), $3.17(\mathrm{~s}, 9 \mathrm{H}), 2.50$ -2.29 (m, 2H), $1.60-1.24(\mathrm{~m}, 2 \mathrm{H}) ;{ }^{1} \mathrm{H}$ NMR $\left(400 \mathrm{MHz}, \mathrm{CDCl}_{3}, 50{ }^{\circ} \mathrm{C}\right) \delta 8.14-7.99$ (m, 1H), $7.99-7.90(\mathrm{~m}, 2 \mathrm{H}), 7.90-7.82(\mathrm{~m}, 1 \mathrm{H}), 7.62-7.46(\mathrm{~m}, 3 \mathrm{H}), 4.97$ - $4.74(\mathrm{~m}$, $2 \mathrm{H}), 4.00-3.67(\mathrm{~m}, 4 \mathrm{H}), 3.18(\mathrm{~s}, 9 \mathrm{H}), 2.59-2.26(\mathrm{~m}, 2 \mathrm{H}), 1.61-1.29(\mathrm{~m}, 2 \mathrm{H}),{ }^{13} \mathrm{C}$ NMR (101 MHz, $\left.\mathrm{CDCl}_{3}\right) \delta 135.2,134.1,133.1,129.9,129.5,128.8,128.1,127.8,127.4$, $123.0,120.8\left(\mathrm{q}, J_{\mathrm{C}-\mathrm{F}}=321.1 \mathrm{~Hz}\right), 102.8,78.9,65.1,65.0,51.8,30.0 ;{ }^{19} \mathrm{~F}$ NMR $(565$ $\mathrm{MHz}, \mathrm{CDCl}_{3}$ ) $\delta-78.3$; FTIR (neat) 3054, 2890, 1489, 1264, 1159, 1031, 830, 639, 518 $\mathrm{cm}^{-1}$; LRMS (ESI+) [M-OTf] $]^{+}$calculated for $\left[\mathrm{C}_{19} \mathrm{H}_{26} \mathrm{NO}_{2}{ }^{+}\right]: 300.2$, found: 300.3. Twodimensonal NMR experiments were used to verify ${ }^{1} \mathrm{H}$ and ${ }^{13} \mathrm{C}$ assignments due to the complex nature of the spectra. 


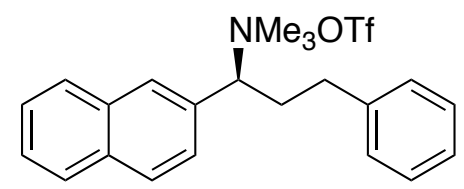

\section{(S)- $N, N, N$-trimethyl-1-(naphthalen-2-yl)-3-phenylpropan-1-aminium}

trifluoromethanesulfonate (1j). Prepared according to General Procedure D on a 1.93 mmol scale from (S)-N,N-dimethyl-1-(naphthalen-2-yl)-3-phenylpropan-1-amine, which was prepared using Escheweiler-Clarke conditions ${ }^{3 a}$ from (S)-3-(1,3-dioxolan-2-yl)-1(naphthalen-2-yl)propan-1-amine (prepared using Ellman's auxiliary ${ }^{2}$ ). In this case, a white precipitate did not form upon addition of MeOTf. Instead, two distinct layers formed. The top layer was decanted off. The bottom layer was washed with $\mathrm{Et}_{2} \mathrm{O}(5 \times 2$ $\mathrm{mL}$ ) and then hexanes ( $5 \times 2 \mathrm{~mL}$, HPLC grade) and then dried under high vacuum to give salt $1 \mathbf{j}(0.854 \mathrm{~g}, 98 \%)$ as a beige solid that slowly turned yellow (mp 65-68 $\left.{ }^{\circ} \mathrm{C}\right):{ }^{1} \mathrm{H}$ NMR $\left(600 \mathrm{MHz}, \mathrm{CDCl}_{3}\right) \delta 8.07-7.92(\mathrm{~m}, 3 \mathrm{H}), 7.90(\mathrm{~d}, J=7.8 \mathrm{~Hz}, 1 \mathrm{H}), 7.64-7.56(\mathrm{~m}, 2 \mathrm{H})$, 7.53 (s, 1H), 7.22 (t, $J=7.5 \mathrm{~Hz}, 2 \mathrm{H}), 7.14$ (t, $J=7.3 \mathrm{~Hz}, 1 \mathrm{H}), 7.05$ (d, $J=7.3 \mathrm{~Hz}, 2 \mathrm{H}$ ), $4.78-4.65(\mathrm{~m}, 1 \mathrm{H}$. Please note: this peak is contaminated with an unknown impurity; however at $50{ }^{\circ} \mathrm{C}$ the peak corresponding to the impurity shifts and a more accurate integration is obtained.), $3.12(\mathrm{~s}, 9 \mathrm{H}), 2.60(\mathrm{~d}, J=6.7 \mathrm{~Hz}, 2 \mathrm{H}), 2.49-2.27(\mathrm{~m}, 2 \mathrm{H}) ;{ }^{1} \mathrm{H}$ NMR (400 MHz, $\left.\mathrm{CDCl}_{3}, 50^{\circ} \mathrm{C}\right) \delta 8.05(\mathrm{~s}, 1 \mathrm{H}), 8.02-7.93(\mathrm{~m}, 2 \mathrm{H}), 7.93-7.87(\mathrm{~m}, 1 \mathrm{H})$, $7.64-7.55(\mathrm{~m}, 2 \mathrm{H}), 7.55-7.50(\mathrm{~m}, 1 \mathrm{H}), 7.25-7.18(\mathrm{~m}, 2 \mathrm{H}), 7.17-7.11(\mathrm{~m}, 1 \mathrm{H}), 7.08$ $-7.02(\mathrm{~m}, 2 \mathrm{H}), 4.77-4.60(\mathrm{~m}, 1 \mathrm{H}), 3.13(\mathrm{~s}, 9 \mathrm{H}), 2.66-2.55(\mathrm{~m}, 2 \mathrm{H}), 2.51-2.33(\mathrm{~m}$, $2 \mathrm{H}) ;{ }^{13} \mathrm{C}$ NMR $\left(101 \mathrm{MHz}, \mathrm{CDCl}_{3}\right) \delta 139.6,135.1,134.1,133.1,130.0,129.5,128.8$, $128.4,128.2,127.9,127.5,127.3,126.7,123.0,120.8\left(\mathrm{q}, J_{\mathrm{C}-\mathrm{F}}=320.9 \mathrm{~Hz}\right), 78.9,51.7$, $32.3,29.3 ;{ }^{19} \mathrm{~F}$ NMR $\left(565 \mathrm{MHz}, \mathrm{CDCl}_{3}\right) \delta-78.3$; FTIR (neat) 3058, 2969, 1490, 1262, $1160,1030,829,638 \mathrm{~cm}^{-1}$; LRMS (ESI+) $[\mathrm{M}-\mathrm{OTf}]^{+}$calculated for $\left[\mathrm{C}_{22} \mathrm{H}_{26} \mathrm{~N}^{+}\right]: 304.2$, found: 304.3. Two-dimensonal NMR experiments were used to verify ${ }^{1} \mathrm{H}$ and ${ }^{13} \mathrm{C}$ assignments due to the complex nature of the spectra.

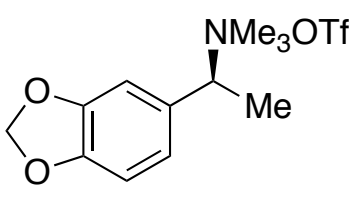




\section{(S)-1-(benzo[d][1,3]dioxol-5-yl)- $N, N, N$-trimethylethanaminium}

trifluoromethanesulfonate (1p). Prepared according to General Procedure D on a 1.52 mmol scale from (S)-1-(benzo[d][1,3]dioxol-5-yl)- $N, N$-dimethylethanamine, which was prepared using Escheweiler-Clarke conditions ${ }^{3 a}$ from $(S)$-1-(benzo[d][1,3]dioxol-5yl)ethanamine (prepared using Ellman's auxiliary $\left.{ }^{2}\right)$, to afford salt $1 \mathbf{p}(0.471 \mathrm{~g}, 87 \%)$ as an off-white solid (mp 136-138 $\left.{ }^{\circ} \mathrm{C}\right) ;{ }^{1} \mathrm{H}$ NMR $\left(600 \mathrm{MHz}, \mathrm{CDCl}_{3}\right) \delta 7.01(\mathrm{~d}, J=7.8 \mathrm{~Hz}, 1 \mathrm{H})$, 6.95 (s, 1H), 6.85 (d, $J=8.0 \mathrm{~Hz}, 1 \mathrm{H}), 6.01(\mathrm{~s}, 2 \mathrm{H}), 4.80$ (q, $J=7.0 \mathrm{~Hz}, 1 \mathrm{H}), 3.11$ (s, 9H), $1.76(\mathrm{~d}, J=6.9 \mathrm{~Hz}, 3 \mathrm{H}) ;{ }^{13} \mathrm{C} \mathrm{NMR}\left(151 \mathrm{MHz}, \mathrm{CDCl}_{3}\right) \delta 149.8,148.6,125.9,120.9(\mathrm{q}$, $\left.J_{\mathrm{C}-\mathrm{F}}=320.1 \mathrm{~Hz}\right), 109.0,102.1,74.1,51.13,51.11,51.08,15.3 ;{ }^{17}{ }^{19} \mathrm{~F}$ NMR $(565 \mathrm{MHz}$, $\left.\mathrm{CDCl}_{3}\right) \delta-78.5$; FTIR (neat) 3045, 2909, 1493, 1256, 1159, 1031, 835, $639 \mathrm{~cm}^{-1}$; LRMS $(\mathrm{ESI}+)[\mathrm{M}-\mathrm{OTf}]^{+}$calculated for $\left[\mathrm{C}_{12} \mathrm{H}_{18} \mathrm{NO}_{2}{ }^{+}\right]$: 208.1, found: 208.2.

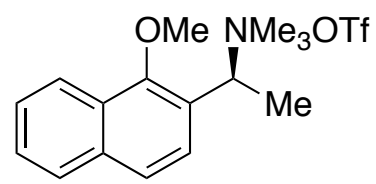

\section{(S)-1-(1-methoxynaphthalen-2-yl)-N,N,N-trimethylethanaminium}

trifluoromethanesulfonate (1q) Prepared according to General Procedure D on a 5.64 mmol scale from (S)-1-(1-methoxynaphthalen-2-yl)- $N, N$-dimethylethanamine, which was prepared using Escheweiler-Clarke conditions ${ }^{3 a}$ from (S)-1-(1-methoxynaphthalen-2yl)ethanamine (prepared using Ellman's auxiliary $\left.{ }^{2}\right)$, to afford salt $\mathbf{1 l}(2.085 \mathrm{~g}, 94 \%)$ as a white solid (mp 123-124 $\left.{ }^{\circ} \mathrm{C}\right) ;{ }^{1} \mathrm{H}$ NMR $\left(600 \mathrm{MHz}, \mathrm{CDCl}_{3}\right) \delta 8.14-8.09(\mathrm{~m}, 1 \mathrm{H}), 7.91-$ $7.86(\mathrm{~m}, 1 \mathrm{H}), 7.74(\mathrm{~d}, J=8.7 \mathrm{~Hz}, 1 \mathrm{H}), 7.62-7.58(\mathrm{~m}, 2 \mathrm{H}), 7.50(\mathrm{~d}, J=8.7 \mathrm{~Hz}, 1 \mathrm{H}), 5.30$ (q, $J=7.1 \mathrm{~Hz}, 1 \mathrm{H}), 4.00(\mathrm{~s}, 3 \mathrm{H}), 3.16(\mathrm{~s}, 9 \mathrm{H}), 1.94(\mathrm{~d}, J=7.0 \mathrm{~Hz}, 3 \mathrm{H}) ;{ }^{13} \mathrm{C}$ NMR $(151$ $\left.\mathrm{MHz}, \mathrm{CDCl}_{3}\right) \delta 156.4,135.9,128.5,128.3,127.5,127.4,125.6,124.4,123.3,120.94$, $120.90\left(\mathrm{q}, J_{\mathrm{C}-\mathrm{F}}=320.1 \mathrm{~Hz}\right), 67.1,63.9,51.38,51.3651 .34,15.4 ;{ }^{17}{ }^{19} \mathrm{~F}$ NMR $(565 \mathrm{MHz}$, $\left.\mathrm{CDCl}_{3}\right) \delta-78.4$; FTIR (neat) 3051, 1471, 1272, 1158, 1031, 827, $639 \mathrm{~cm}^{-1}$; LRMS (ESI+) [M-OTf] ${ }^{+}$calculated for $\left[\mathrm{C}_{16} \mathrm{H}_{22} \mathrm{NO}^{+}\right]$: 244.2, found: 244.2 . 


\section{References}

1. Pangborn, A. B.; Giardello, M. A.; Grubbs, R. H.; Rosen, R. K.; Timmers, F. J. Organometallics 1996, 15, 1518.

2. (a) Procopiou, G.; Lewis, W.; Harbottle, G.; Stockman, R. A. Org. Lett. 2013, 15, 2030; (b) Aggarwal, V. K.; Barbero, N.; McGarrigle, E. M.; Mickle, G.; Navas, R.; Suarez, R.; Unthank, M. G.; Yar, M. Tetrahedron Lett. 2009, 50, 3482; (c) Tanuwidjaja, J.; Peltier, H. M.; Ellman, J. A. J. Org. Chem. 2007, 72, 626.

3. (a) Icke, R. N.; Wisegarver, B. B.; Alles, G. A. Org. Synth. 1955, Coll. Vol. 3, 723;

(b) Borch, R. F.; Hassid, A. I. J. Org. Chem. 1972, 37, 1673.

4. In some cases, the benzylic carbon is not observed to to quadrupolar broadening caused by ${ }^{11} \mathrm{~B}$.

5. Noh, D.; Chea, H.; Ju, J.; Yun, J. Angew. Chem., Int. Ed. 2009, 48, 6062.

6. $\quad$ Ren, X.; Li, G.; Wei, S.; Du, H. Org. Lett. 2015, 17, 990.

7. Crudden, C. M.; Hleba, Y. B.; Chen, A. C. J. Am. Chem. Soc. 2004, 126, 9200.

8. Yadav, J. S.; Nanda, S.; Reddy, P. T.; Rao, A. B. J. Org. Chem. 2002, 67, 3900.

9. Legouin, B.; Gayral, M.; Uriac, P.; Cupif, J.-F.; Levoin, N.; Toupet, L.; van de Weghe, P. Eur. J. Org. Chem. 2010, 2010, 5503.

10. Liu, Y.; Da, C.-S.; Yu, S.-L.; Yin, X.-G.; Wang, J.-R.; Fan, X.-Y.; Li, W.-P.; Wang, R. J. Org. Chem. 2010, 75, 6869.

11. El-Shehawy, A. A.; Sugiyama, K.; Hirao, A. Tetrahedron Asym. 2008, 19, 425.

12. Li, H.; Wang, L.; Zhang, Y.; Wang, J. Angew. Chem., Int. Ed. 2012, 51, 2943.

13. Wei, S.; Du, H. J. Am. Chem. Soc. 2014, 136, 12261.

14. Balieu, S.; Hallett, G. E.; Burns, M.; Bootwicha, T.; Studley, J.; Aggarwal, V. K. J. Am. Chem. Soc. 2015, 137, 4398.

15. Zuo, Z.; Zhang, L.; Leng, X.; Huang, Z. Chem. Commun. 2015, 51, 5073.

16. (a) Maity, P.; Shacklady-McAtee, D. M.; Yap, G. P. A.; Sirianni, E. R.; Watson, M. P. J. Am. Chem. Soc. 2013, 135, 280; (b) Shacklady-McAtee, D. M.; Roberts, K. M.; Basch, C. H.; Song, Y.-G.; Watson, M. P. Tetrahedron 2014, 70, 4257.

17. In several of the ammonium triflates, the methyl groups of the $\mathrm{NMe}_{3}$ fragment appear as three, nearly coincident peaks. We hypothesize that this may be due to hindered rotation about the benzylic $\mathrm{C}-\mathrm{N}$ bond. rotation. 
$\circ$
2

Zน.

St.

29.2

59.2

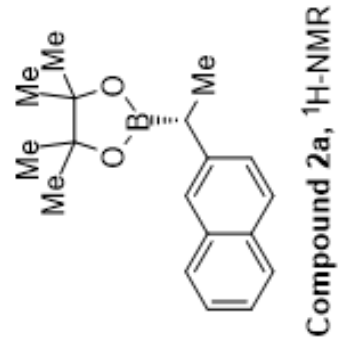

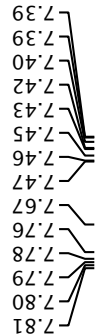

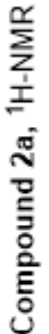




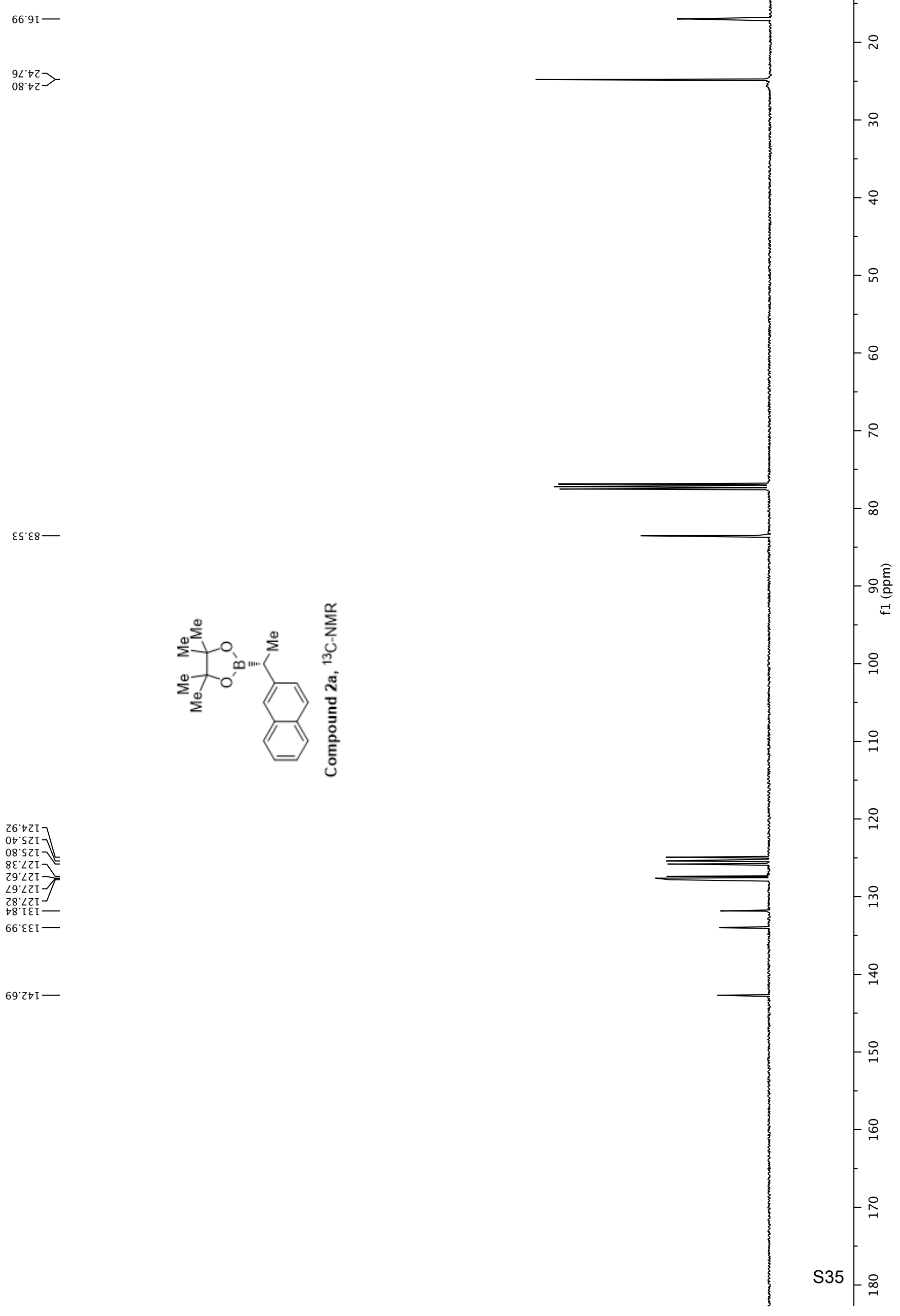



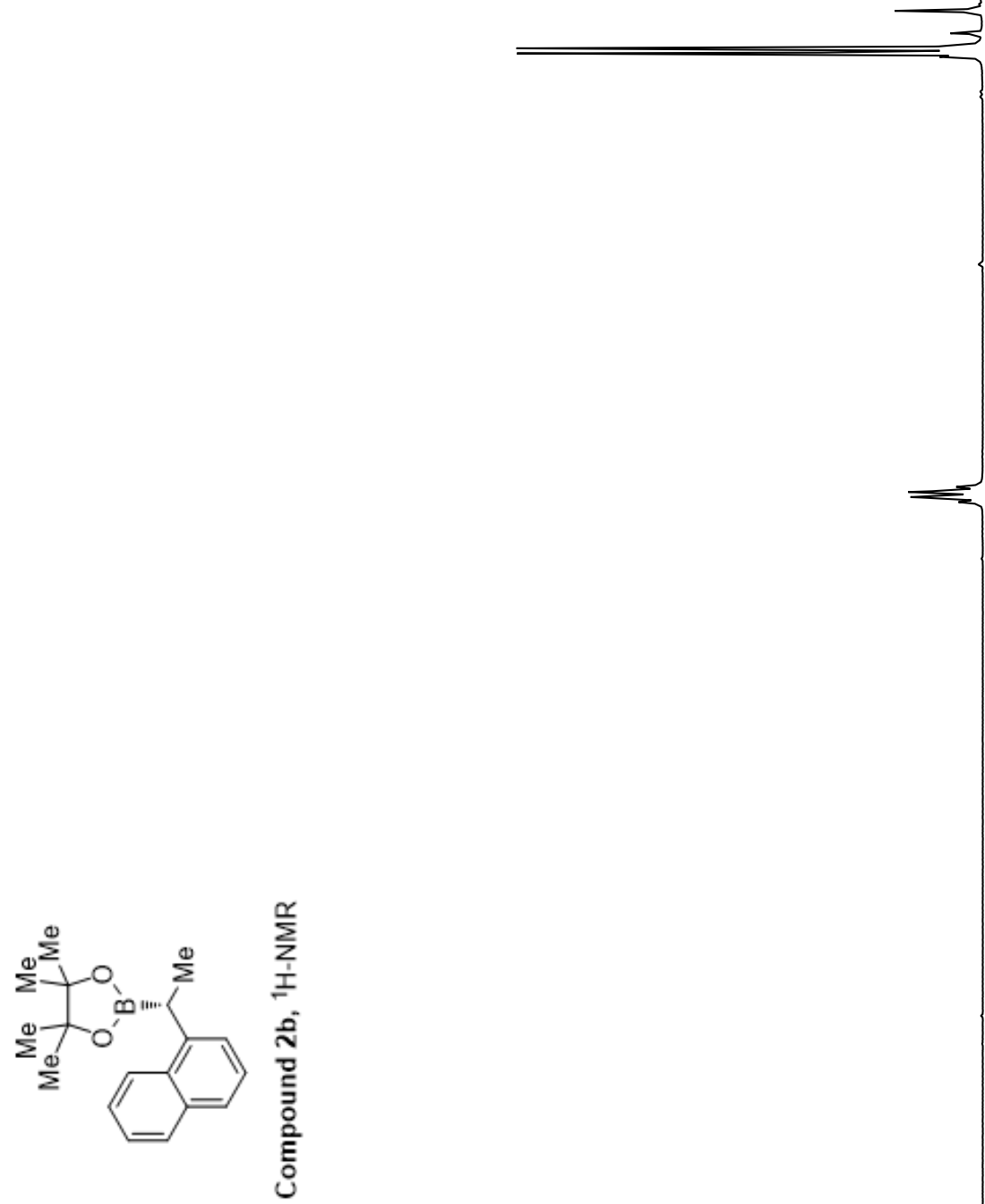
$\angle S^{\circ} 9 \mathrm{I}$

$0<t z>$
$t 8 \cdot t z$

$09^{\circ} \varepsilon 8-$

$02+62[7$

6Eं $\bullet Z I$

ZS.SZI

$00.92 \mathrm{I}$

68.821

हI'حદI -

SO ${ }^{\circ}$ เ I -

$\forall S^{\cdot} I t I$

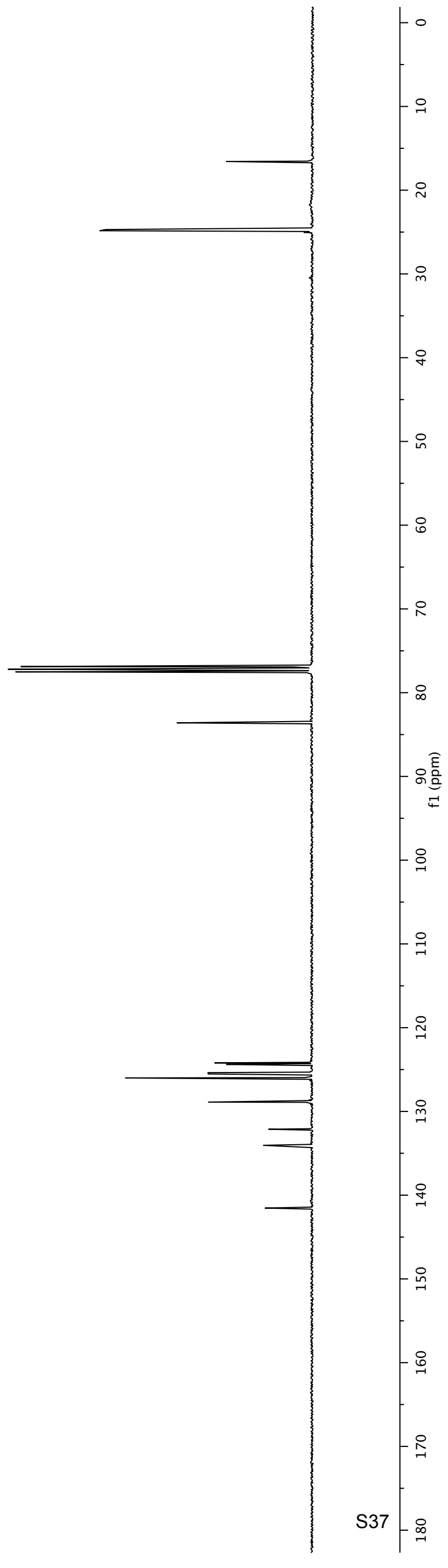

흥

움

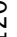

$\stackrel{m}{-}$

옥

요

8

$+\frac{1}{4}$

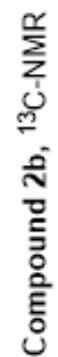




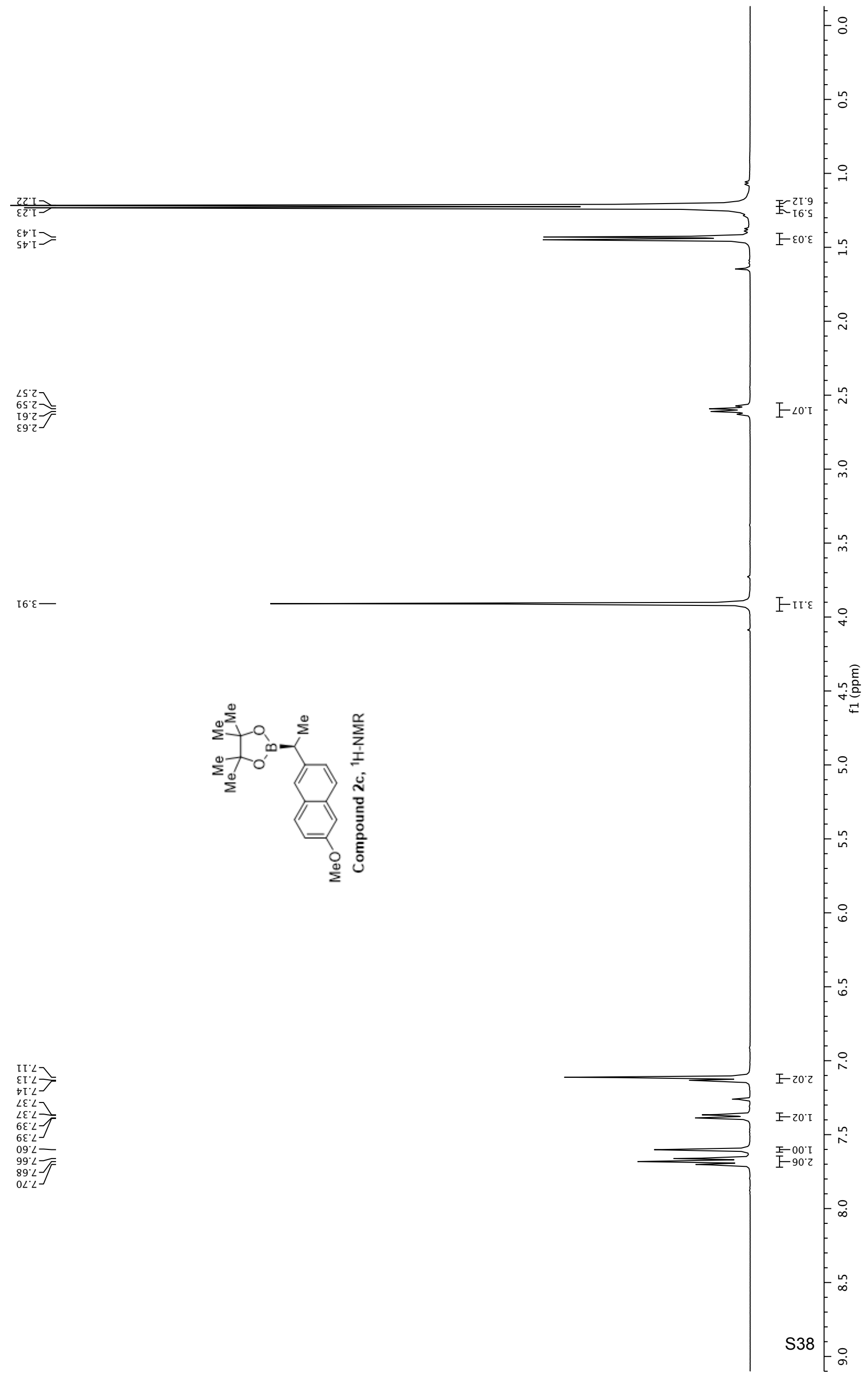



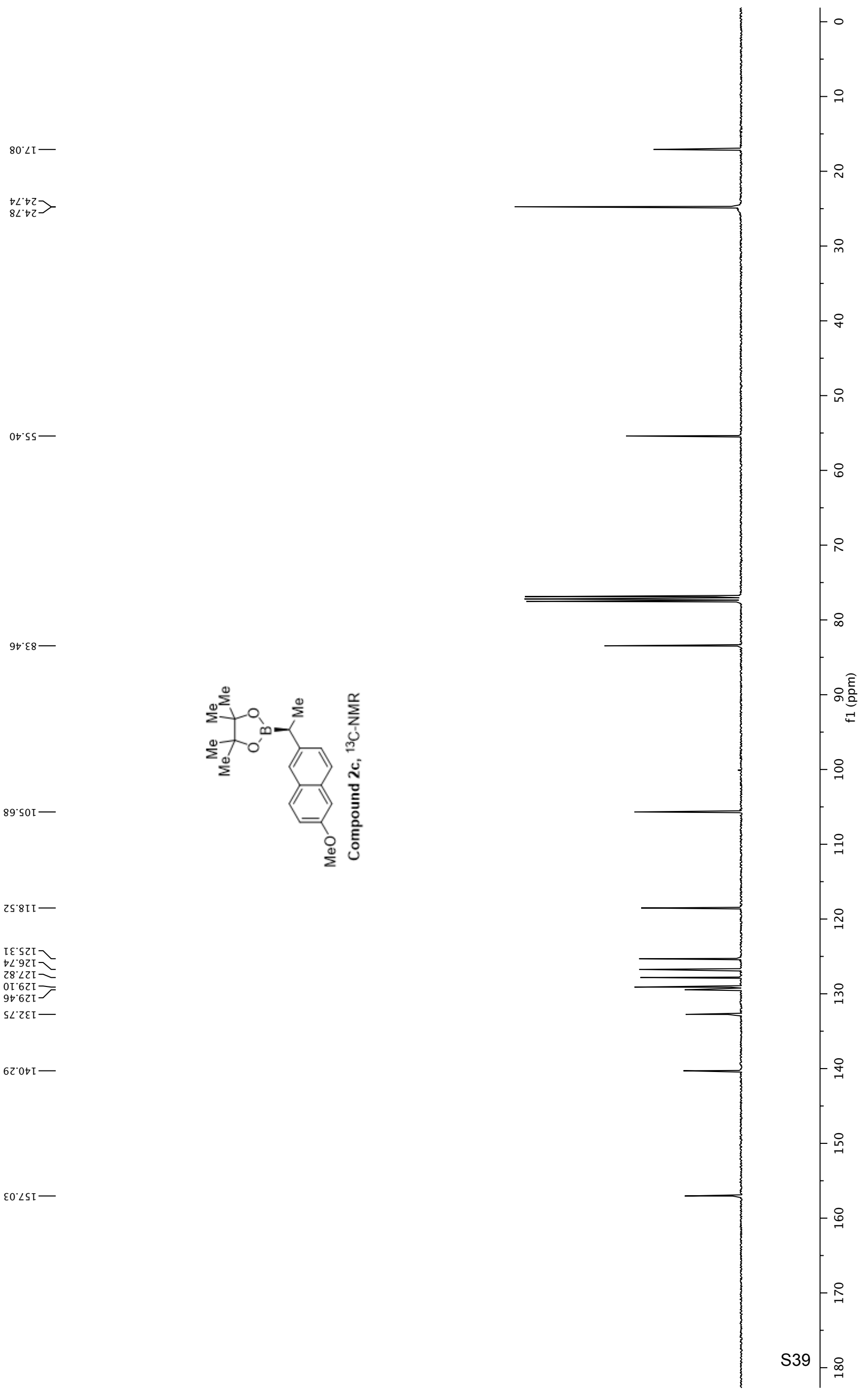
$8 \mathrm{~L}^{\circ} \mathrm{T}$

Sट'โ

It $[\supset$

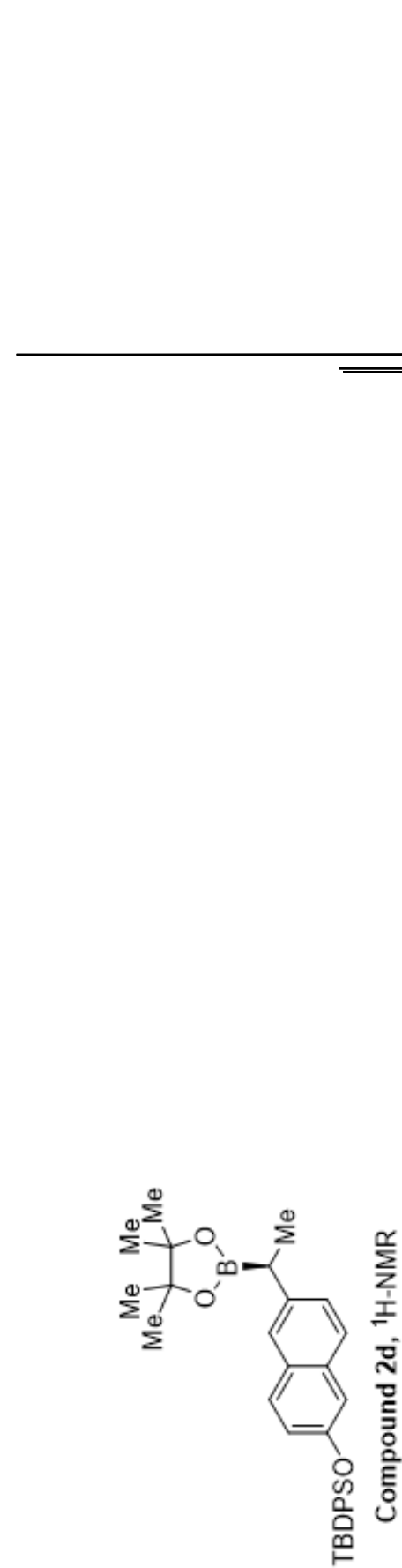

ss. 27

$95^{\circ} 2$

$09.2]$

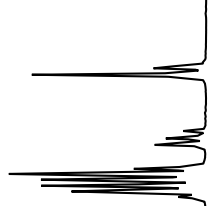

I 002

瓦现,

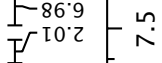

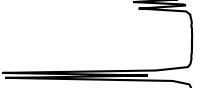

下 $\varepsilon 6^{\circ} \varepsilon$ 
I $Z^{\circ} \angle \mathrm{I}$

$0 L^{\circ} 6 \mathrm{I}$

$6<+b z$
$28 \cdot b z$

9L.92

$97^{\circ} \varepsilon 8-$

OS. tน I

$\angle t \cdot I Z I-$

$8 \mathrm{I} \cdot \mathrm{SZ}]$

SL $92 \mathrm{9}$. $\angle 2 \mathrm{I}$

9522I

82.821

$29.621-$

घ0.0हI

$\forall L ' Z \varepsilon I-$

દZ દદIJ

عャ'0†I -

$8 L ' Z S I$

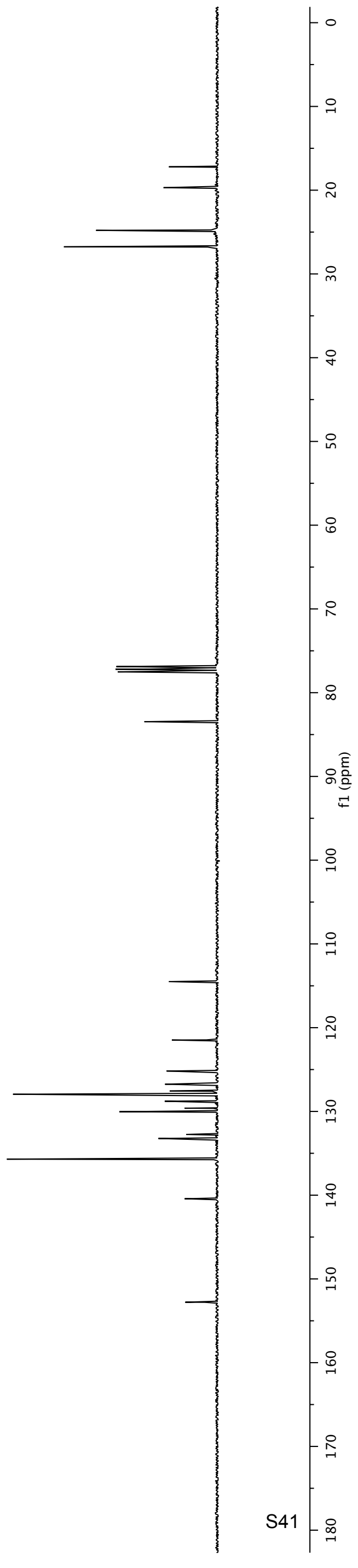




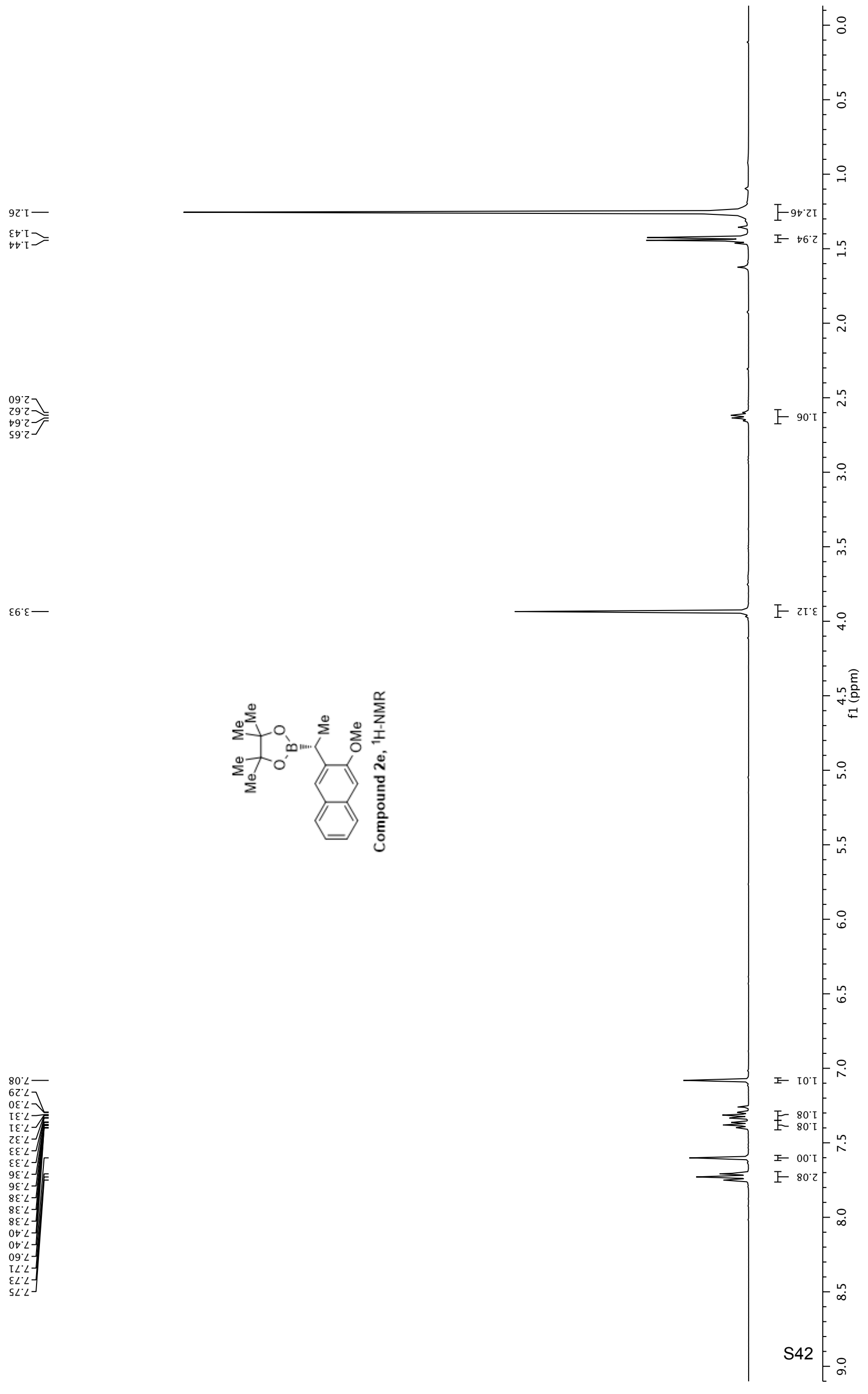



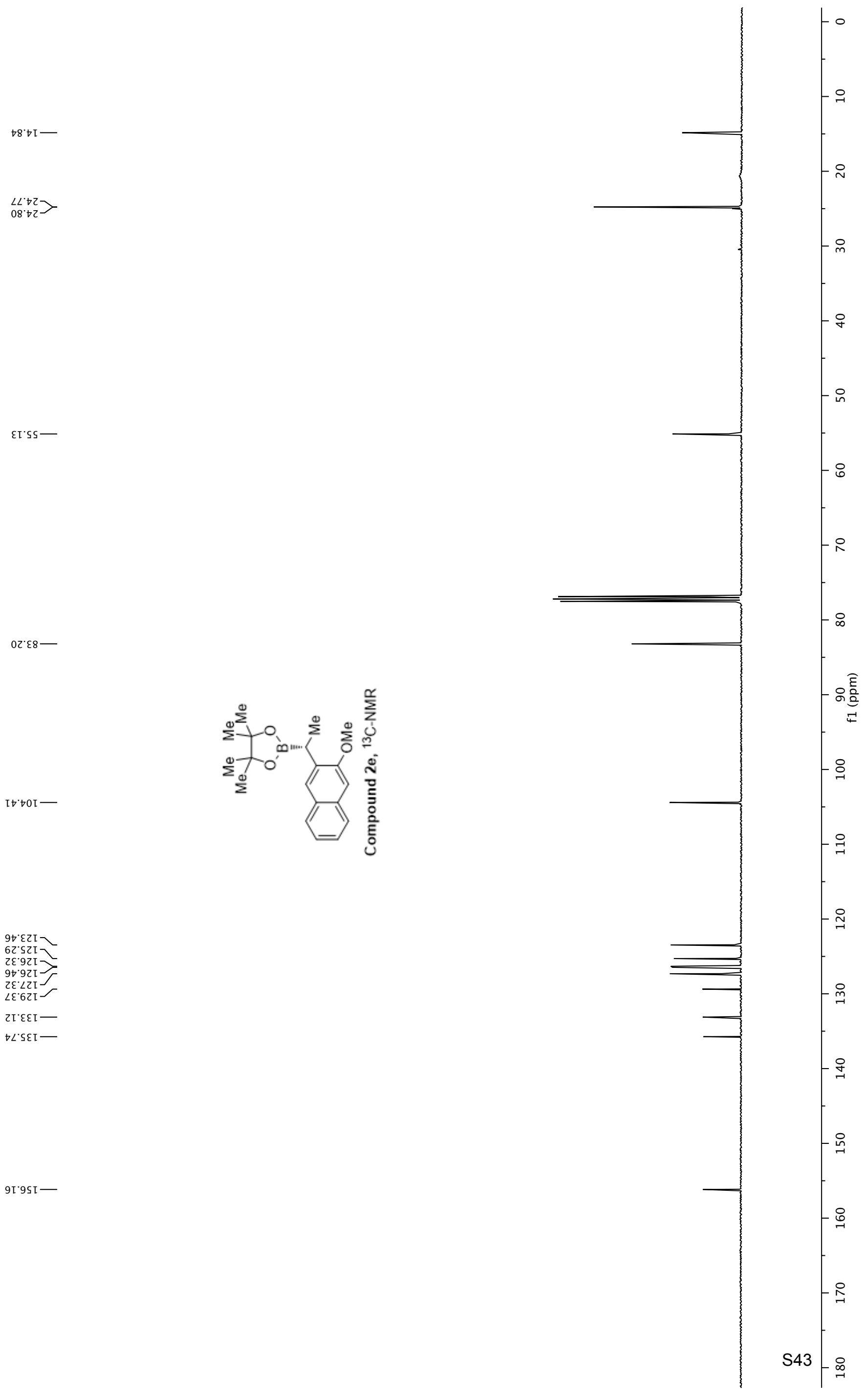

97.દZI

टह.92 \

9t.92

Zहं LZI

$\angle \varepsilon^{\circ} 62 \mathrm{I}$

乙I' $\varepsilon \varepsilon I-$

$\forall L \cdot S E I-$

9I.9SI

ईิ 


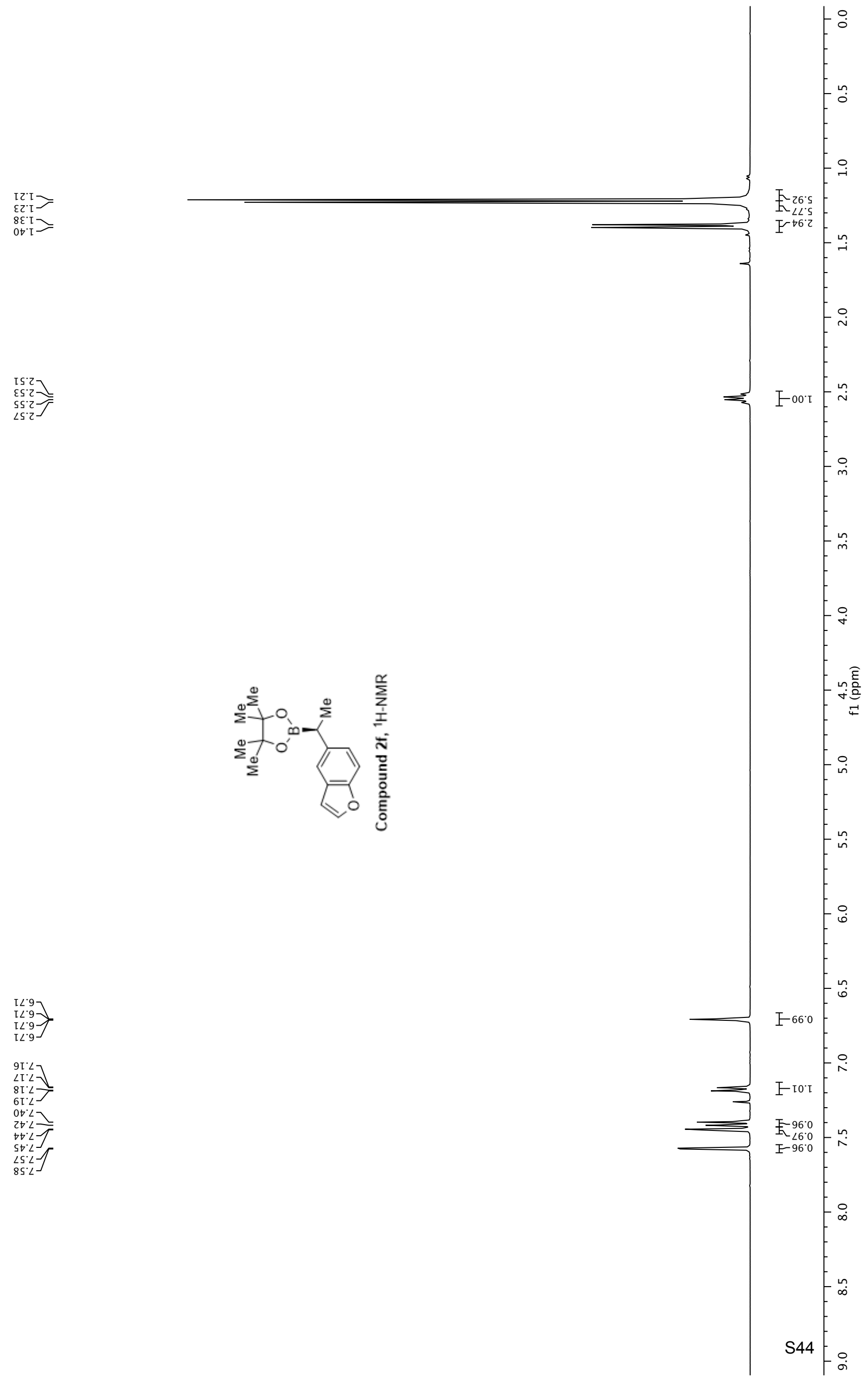




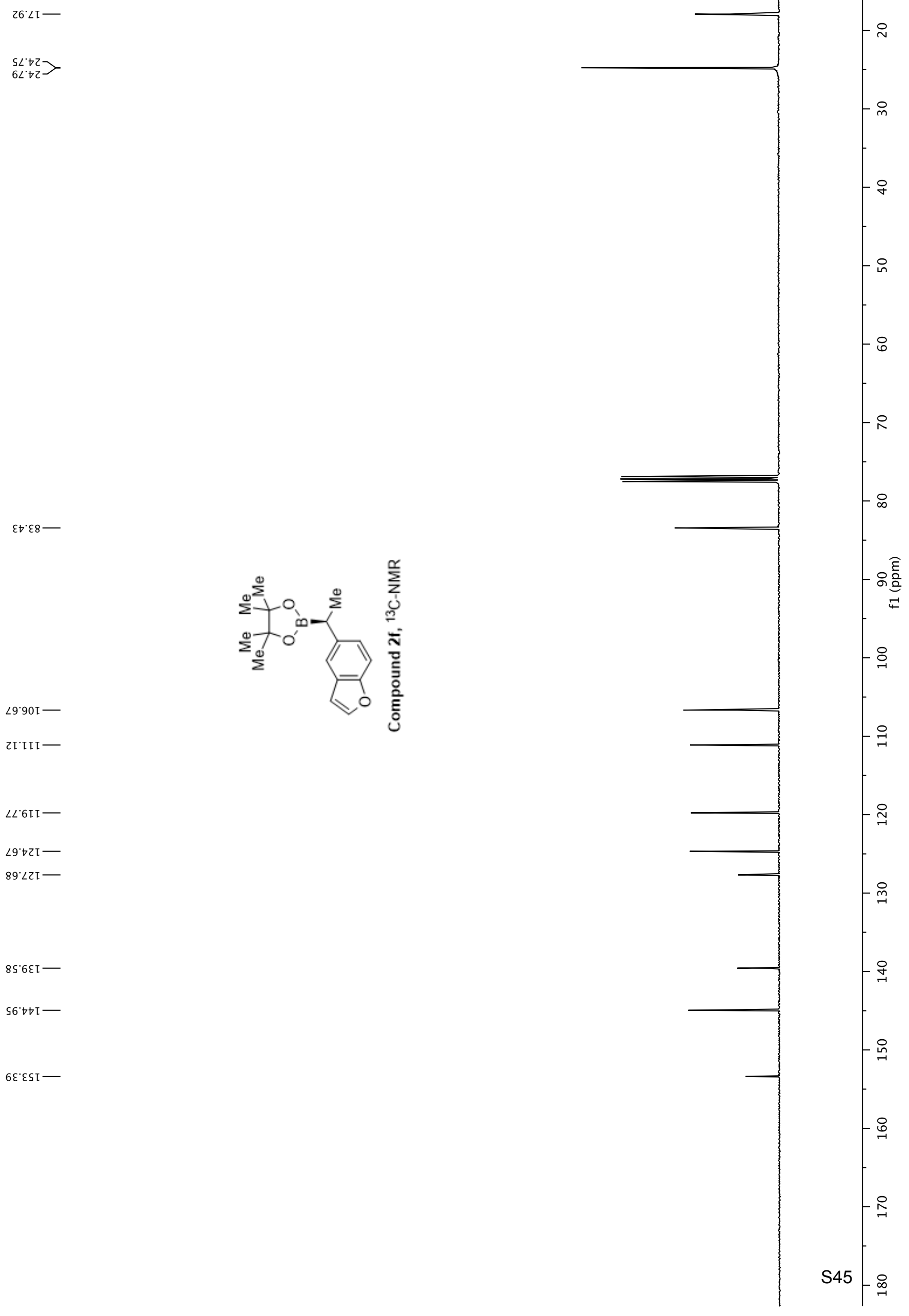


$8 \mathrm{I}^{\circ} \mathrm{T} 工$
$0 \mathrm{~T}$

Zะ'

$\varepsilon \varepsilon^{\cdot}[-$

$\varepsilon \varepsilon^{\prime} 乙$
$9 t^{\circ} 乙$

$9+2]$

$80^{\circ}-1$

$05^{2} 2$

$\left\langle S^{\circ} \cdot\right\rangle$

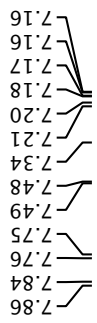

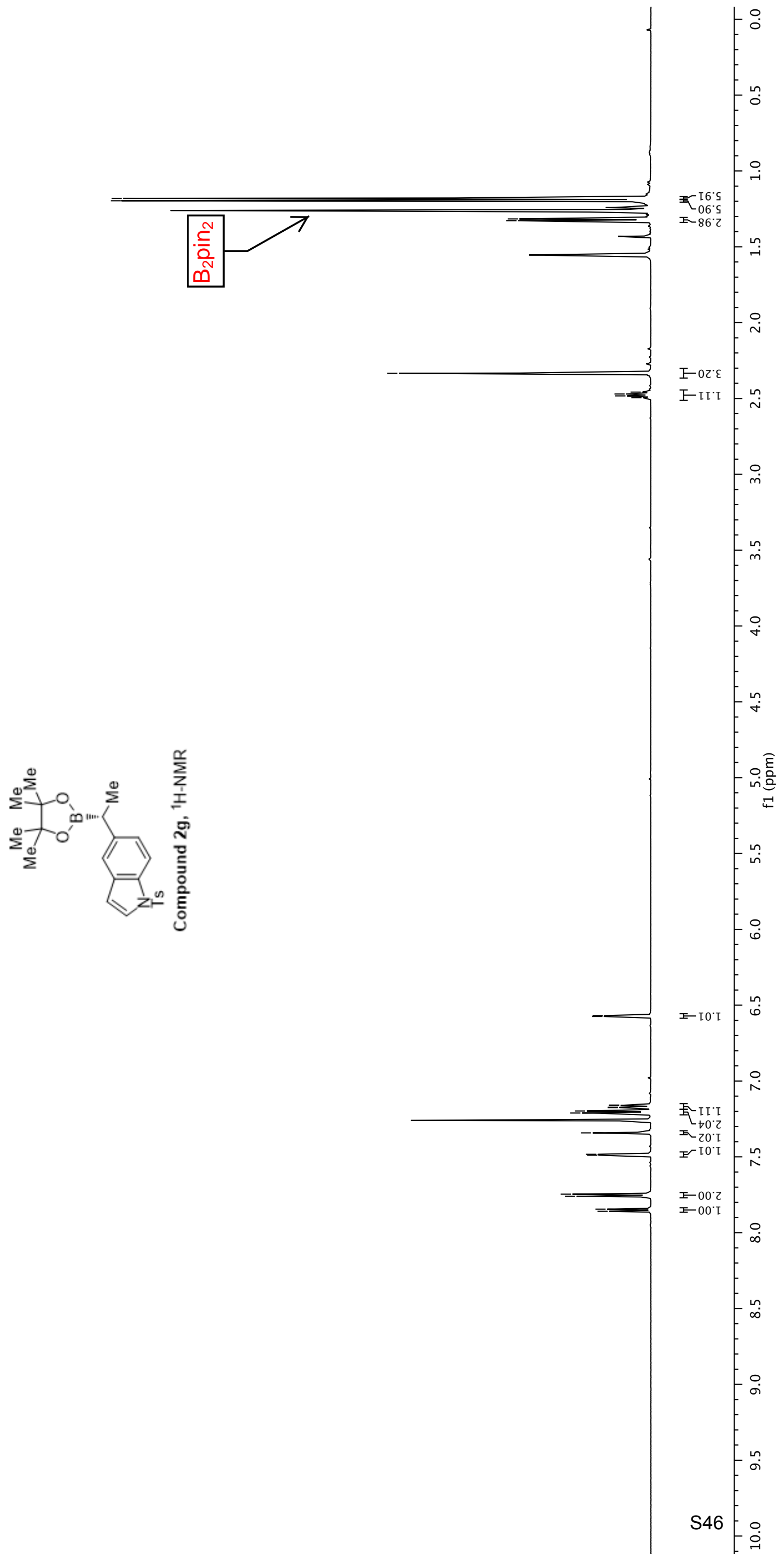




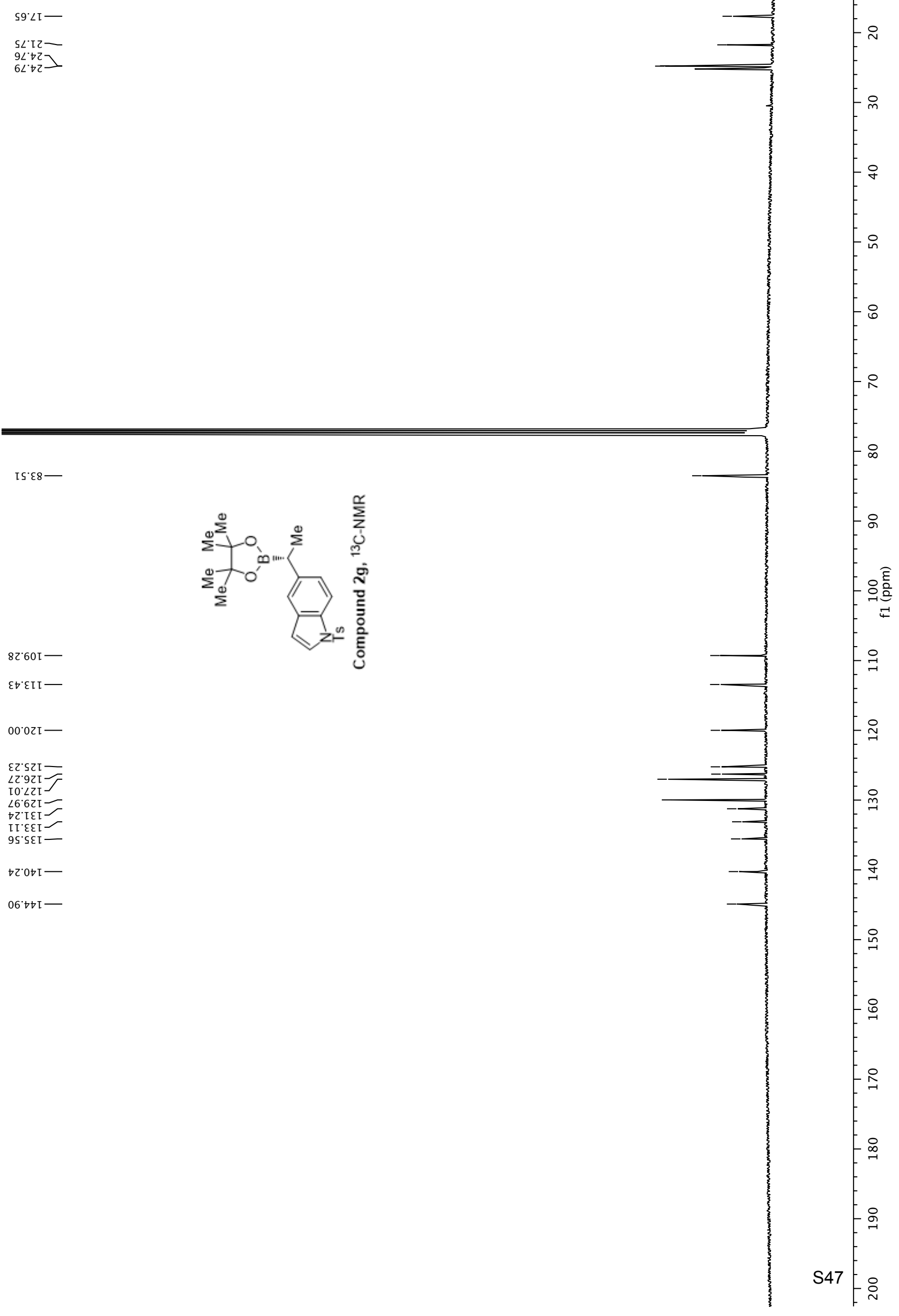




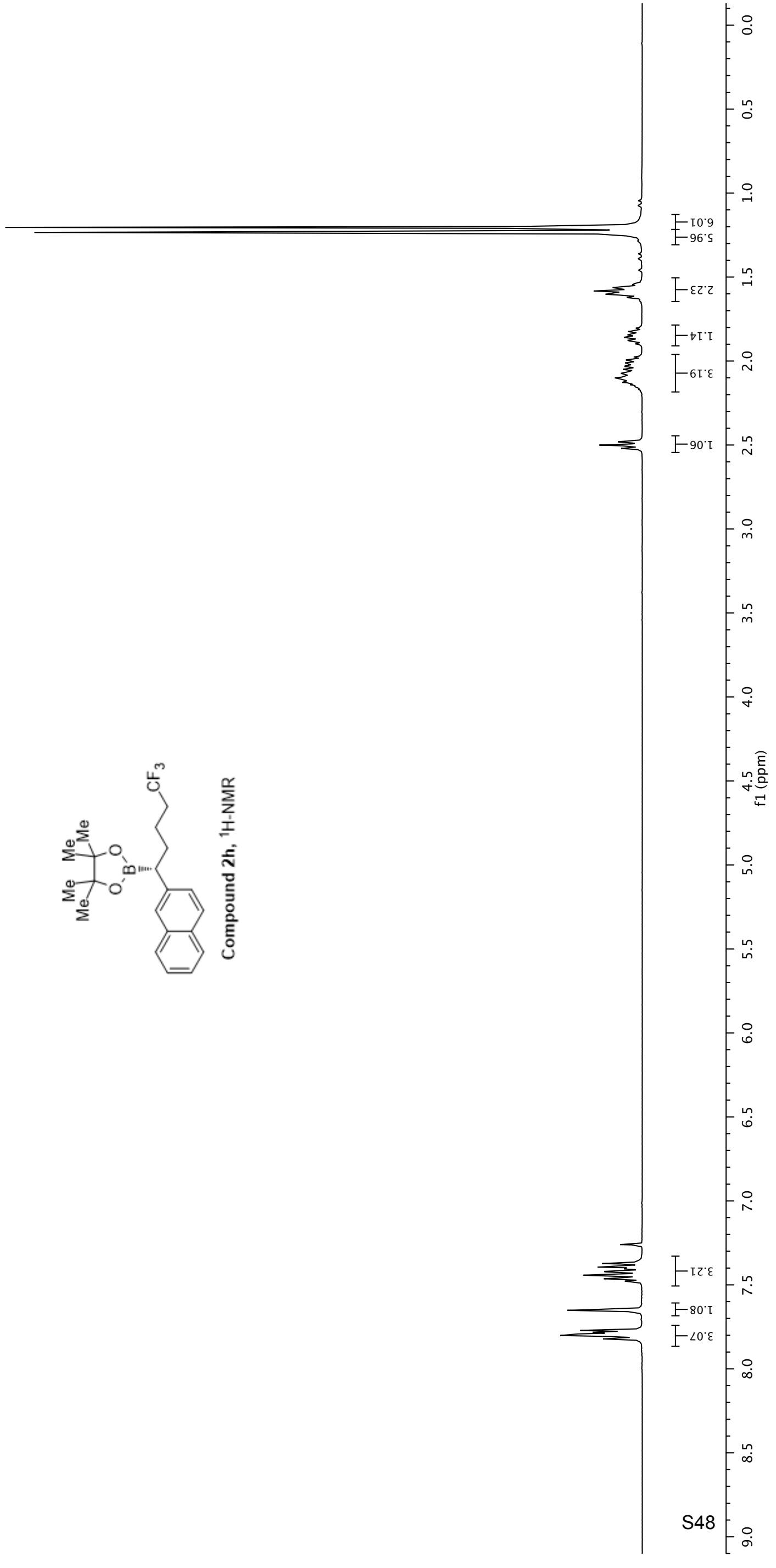


$0<{ }^{\circ}[2]$

$2 L[2]$

$\forall<[2]$

$\varepsilon<\succ z-1$

[8.七2]

6S. $[\varepsilon]$

ธ9.

$\varepsilon 0^{\circ} \downarrow \varepsilon-$

乙乙॰ $\downarrow \varepsilon$

$\downarrow L^{\prime} \varepsilon 8-$
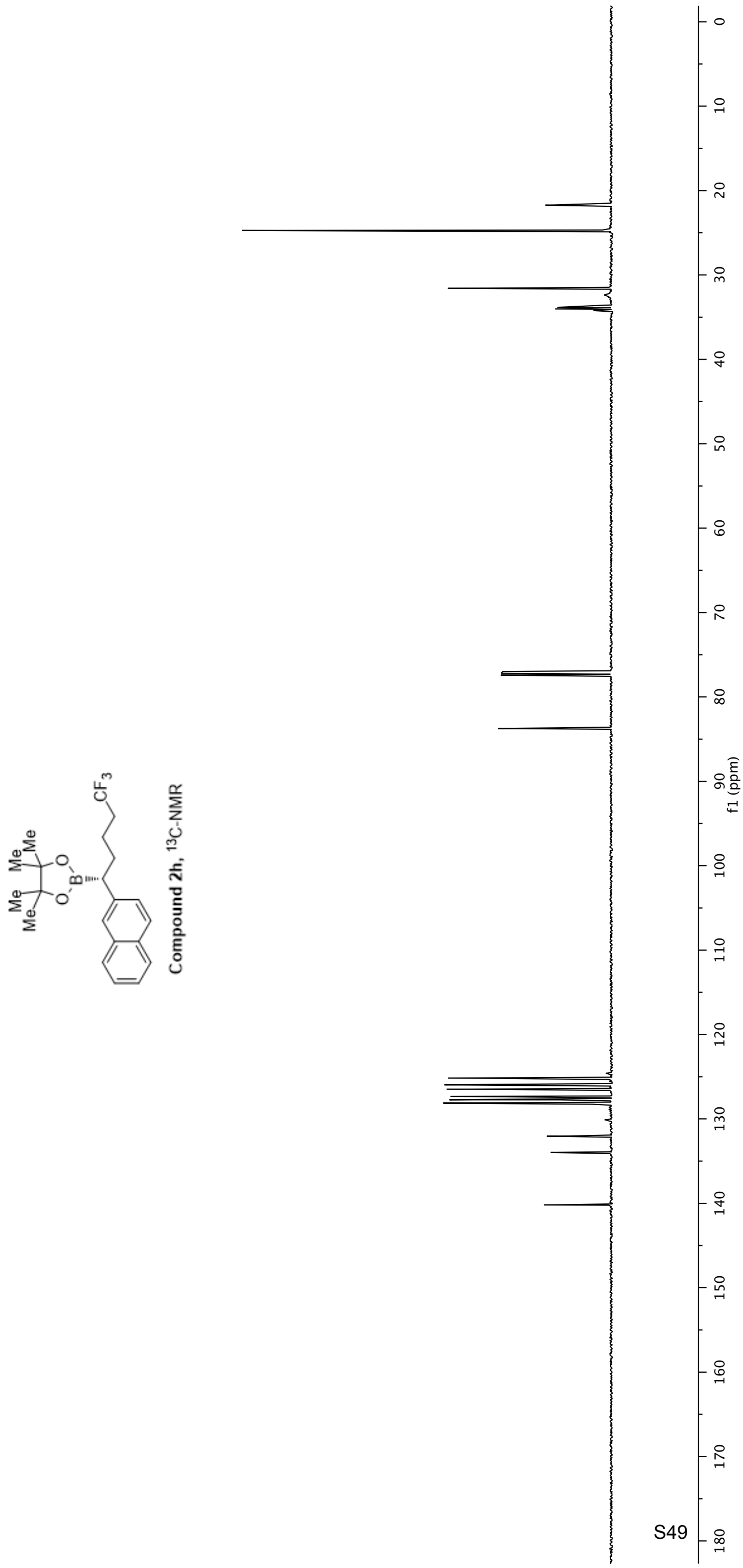

$09{ }^{\circ} \sqcup 2[$

6I.0 


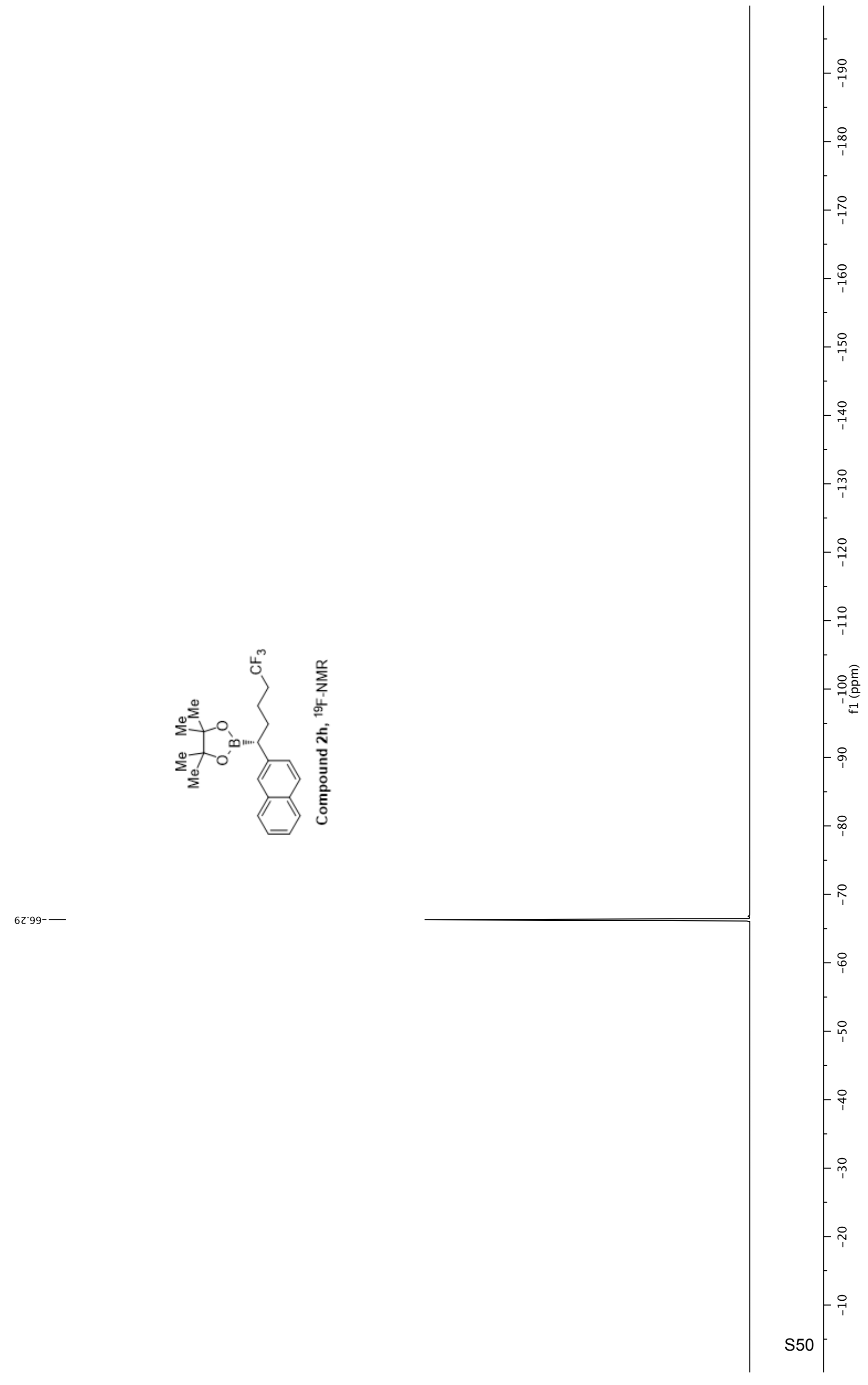




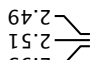

\&S. 2

$8 L^{\circ} \varepsilon$

$6 L^{\prime} \varepsilon-$

$08^{\circ} \varepsilon$
$28^{\circ} \varepsilon-1-$
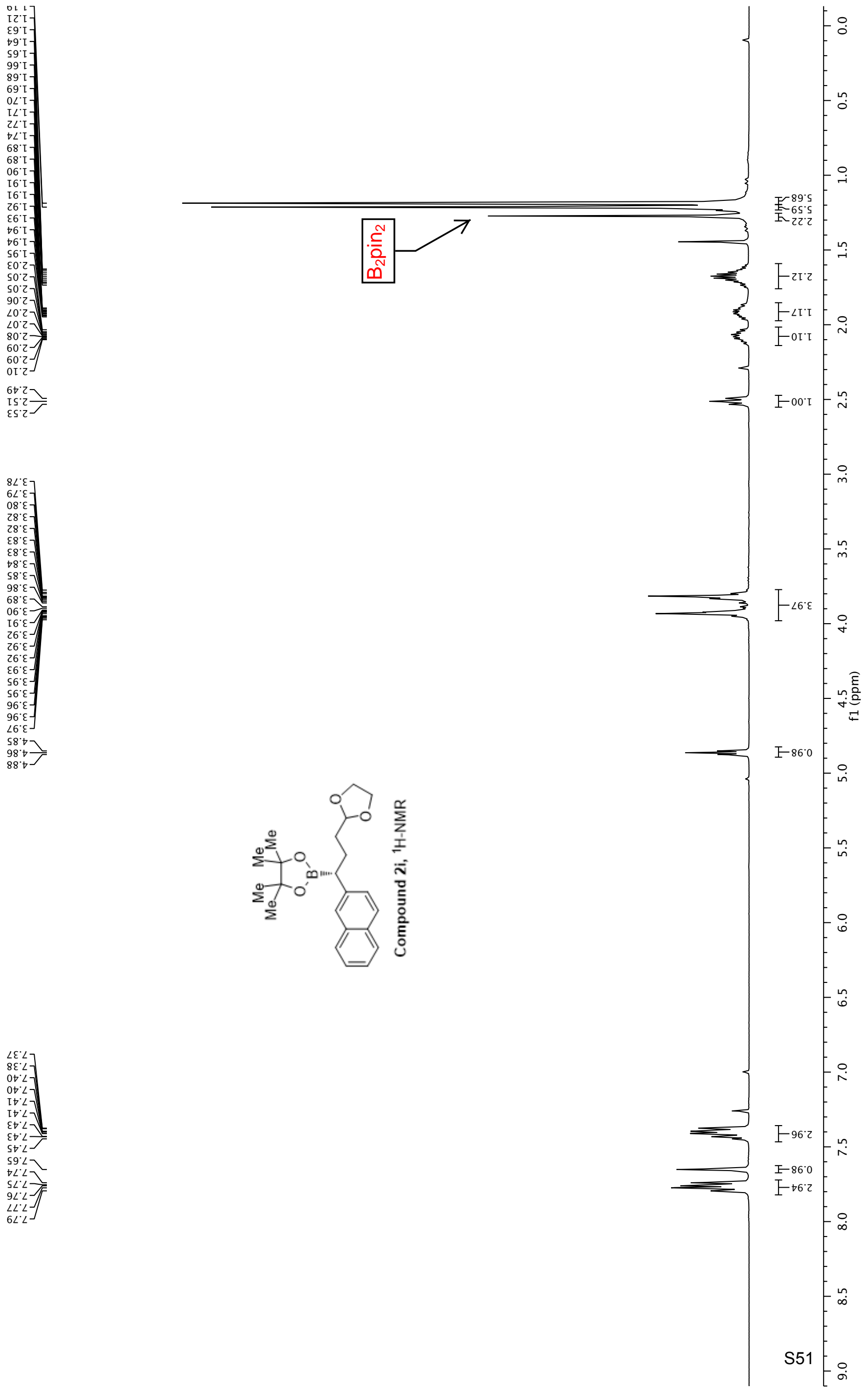


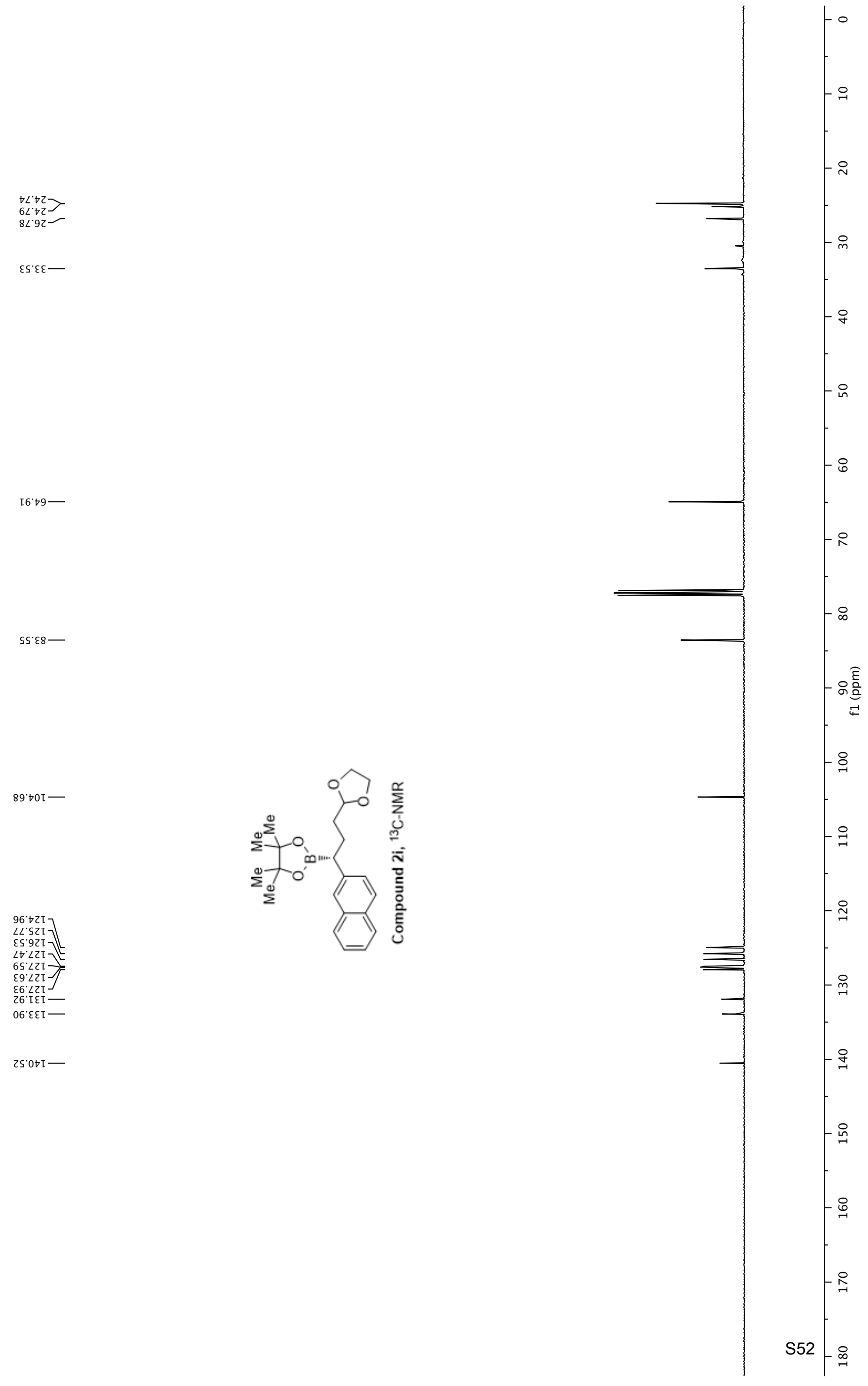




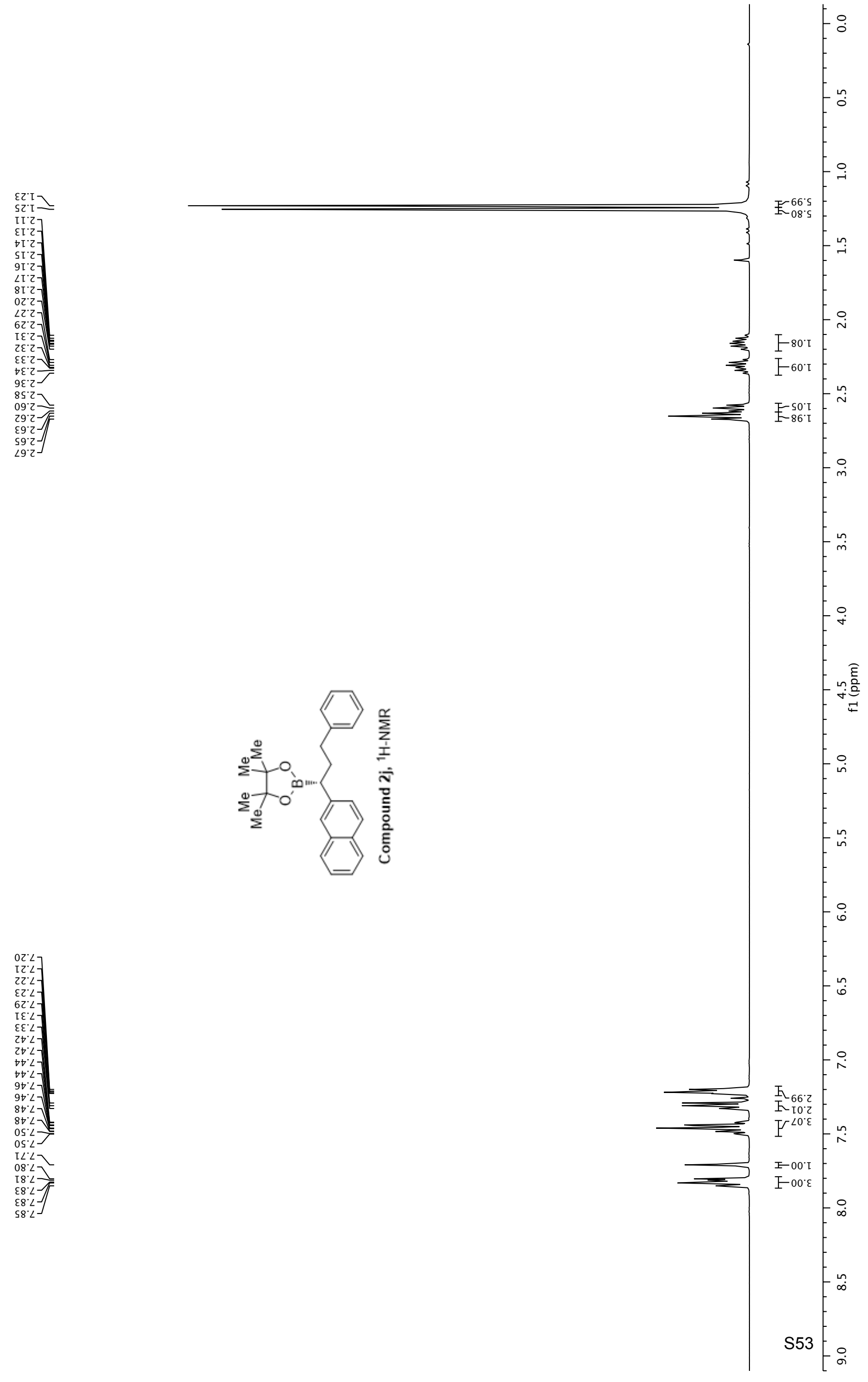



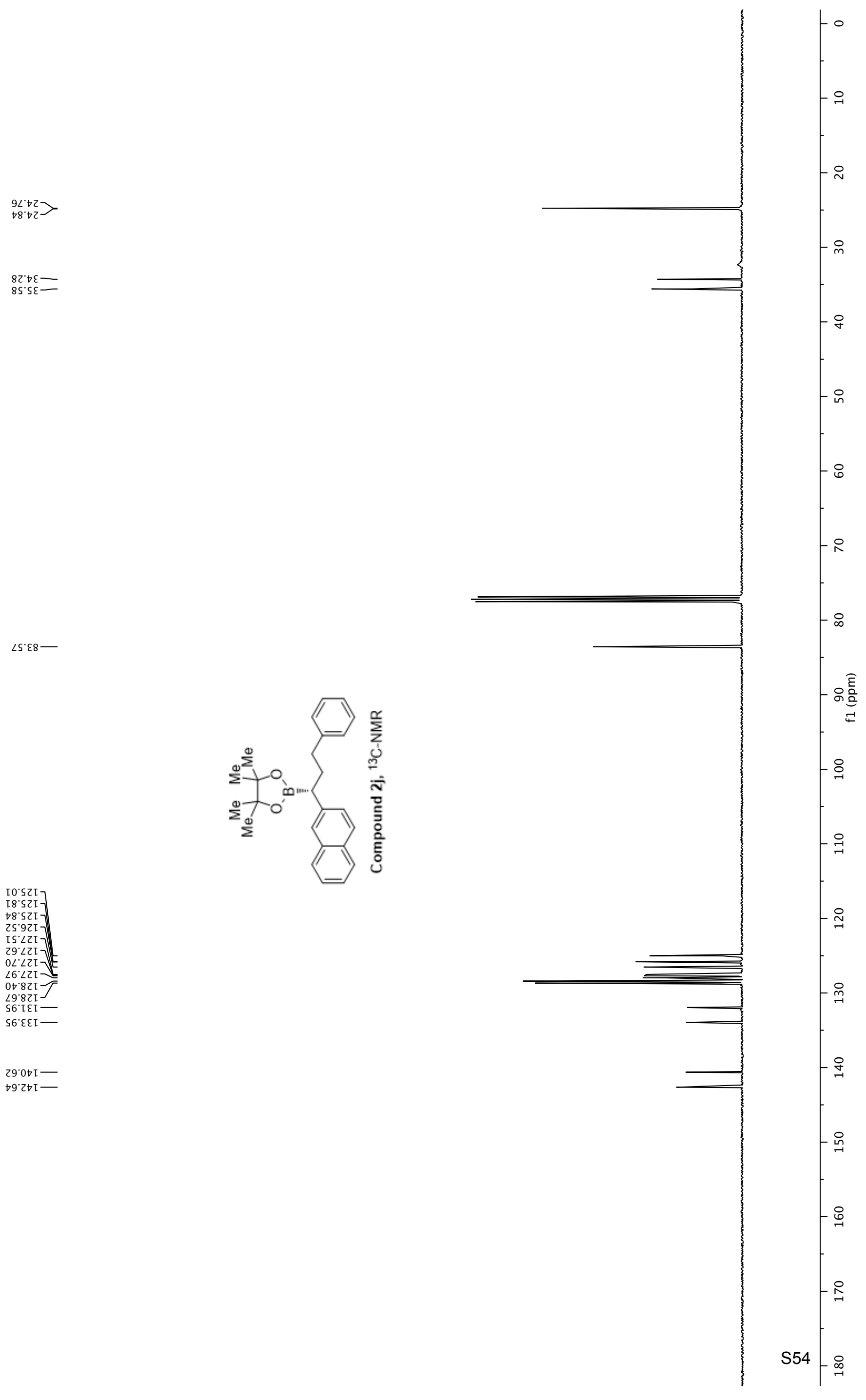
$60^{\circ} \mathrm{I}$

${ }_{8 \mathrm{I}^{\circ} \mathrm{T}} \mathrm{L}$

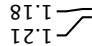

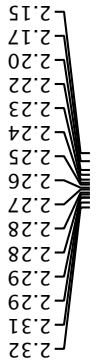
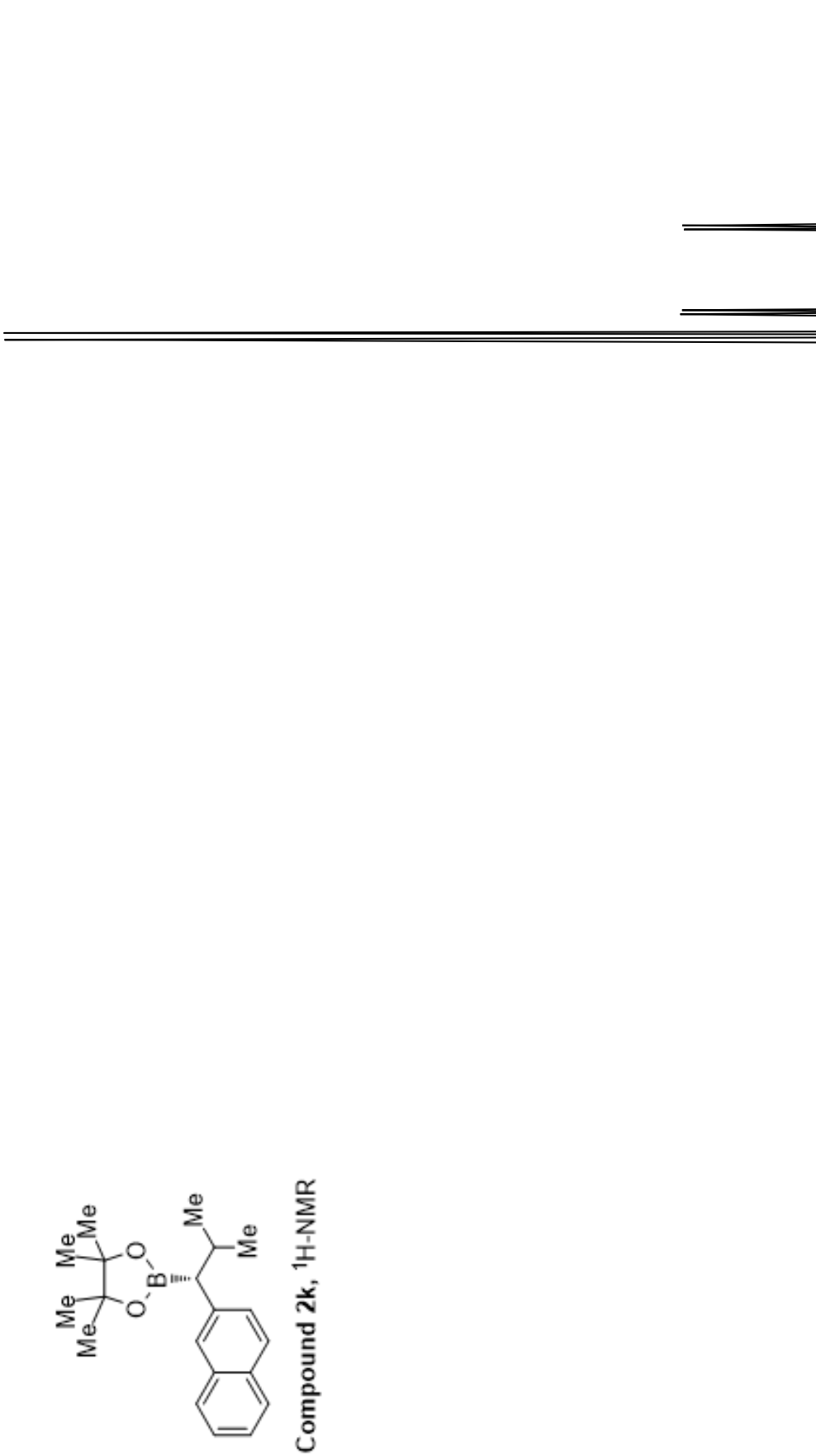

- 웅

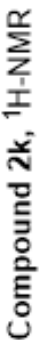
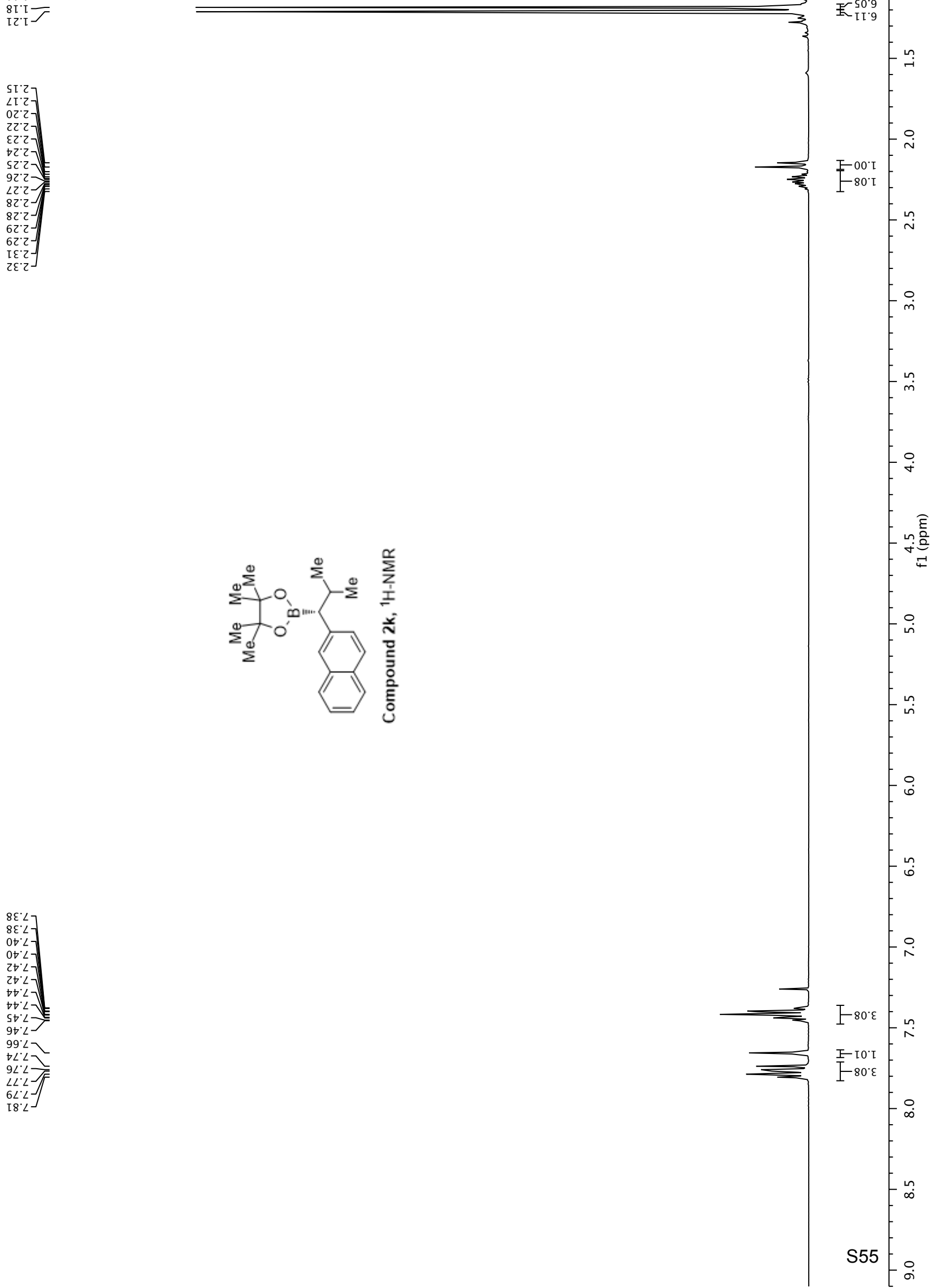
$92 \cdot 22$
$2 t \cdot \varepsilon 2-$

$t<\forall z>$
$\varepsilon 8 \cdot \nabla z$

$60.5 \varepsilon$
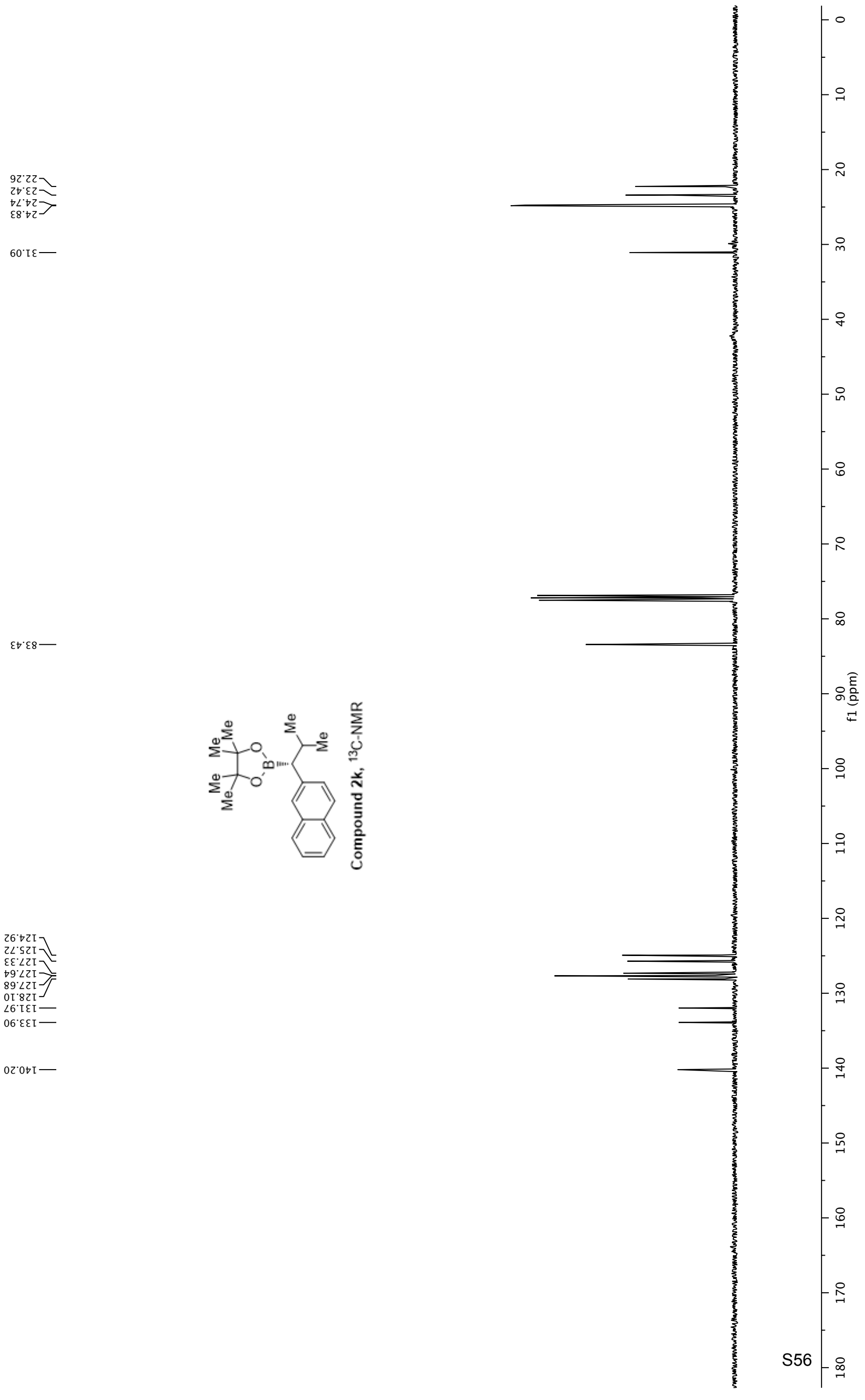

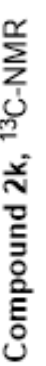

층 을 
OZ.' ${ }^{\circ} \mathrm{L} 工$

$0 \varepsilon^{\prime}[\overline{ }$

68: 27

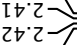

$\Delta t 2$

26.9
26.97

ع6.9]

七6. 97

ธ6.97

56.

96.9

86.9

SI $\angle-$

$9 \mathrm{I} \cdot L$

$9[2-$

$8 \mathrm{I} \cdot \mathrm{L}-$

$6 \mathrm{I}: \mathrm{L}$

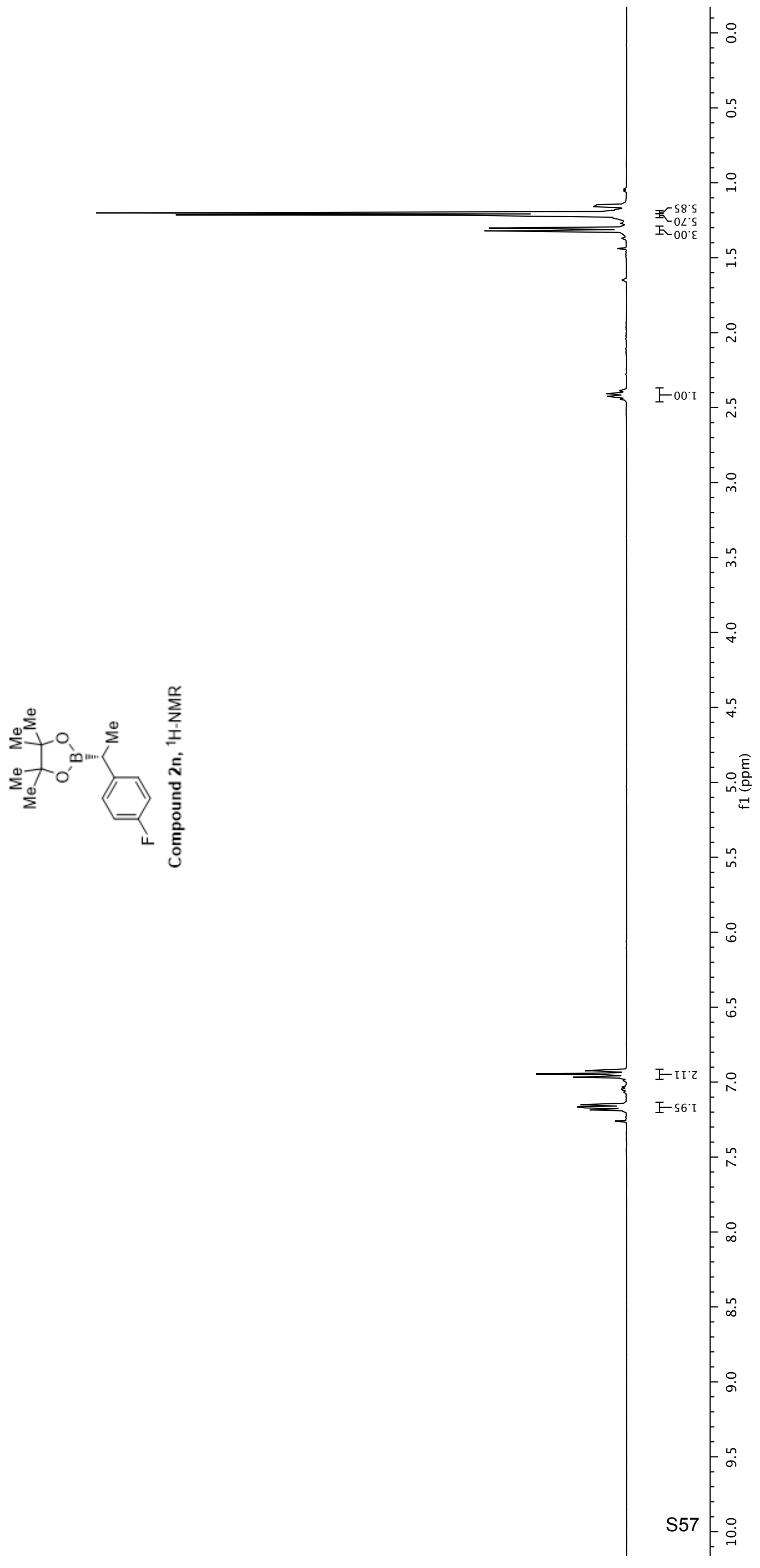



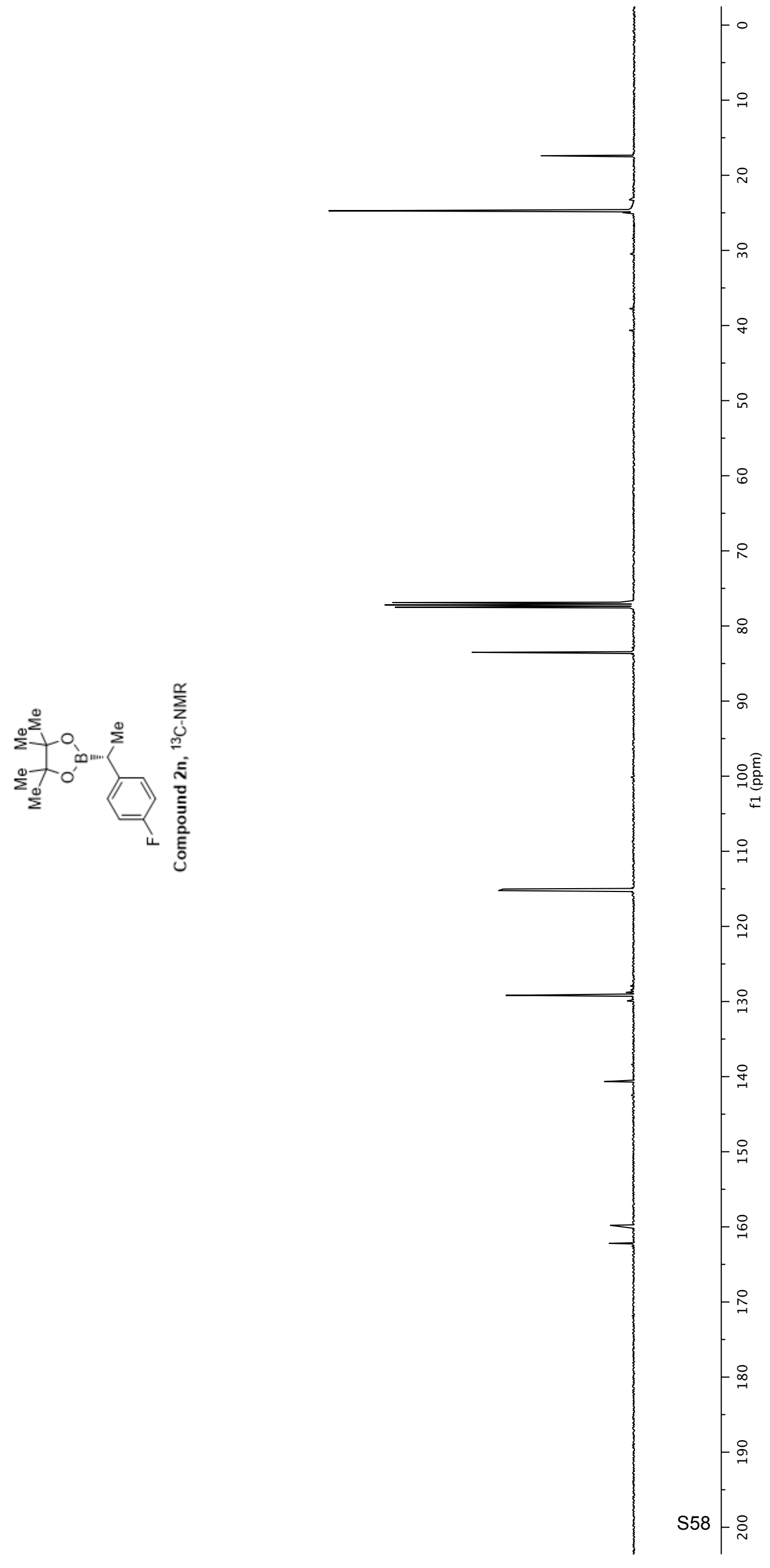

$20 \cdot$ SII
$\varepsilon Z^{\circ}$ SII

$0 I^{\circ} 62 \mathrm{I}$
$8 \mathrm{I}^{\circ} 62 \mathrm{I}$

$\varepsilon 9^{\circ} 0 \downarrow[\succ$

$\varepsilon 9^{\circ} 0 t[$
$99^{\circ} 0 t[$

6L'6SI 02' 29 I 
$0 \mathrm{Z}^{\circ} \mathrm{I}$
$\mathrm{ZZ} \cdot \mathrm{L}$

$62 \cdot 1$

I $\varepsilon^{\prime}$ [

s $\varepsilon^{\prime} \mathrm{Z}$

$\angle \varepsilon \cdot 2$

$6 \varepsilon$
$0+2$

$8 L^{\circ} \varepsilon-$

${ }_{88.9}^{18.9}=$

$\varepsilon \tau \cdot L$
si $\angle$

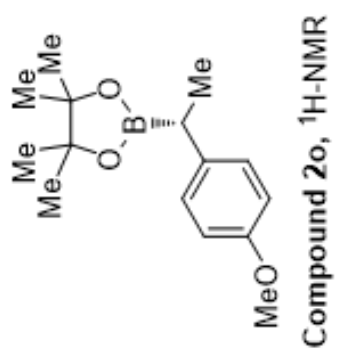


$2 t<L$

$29.72>$

ऽ9.

0Z'SS-
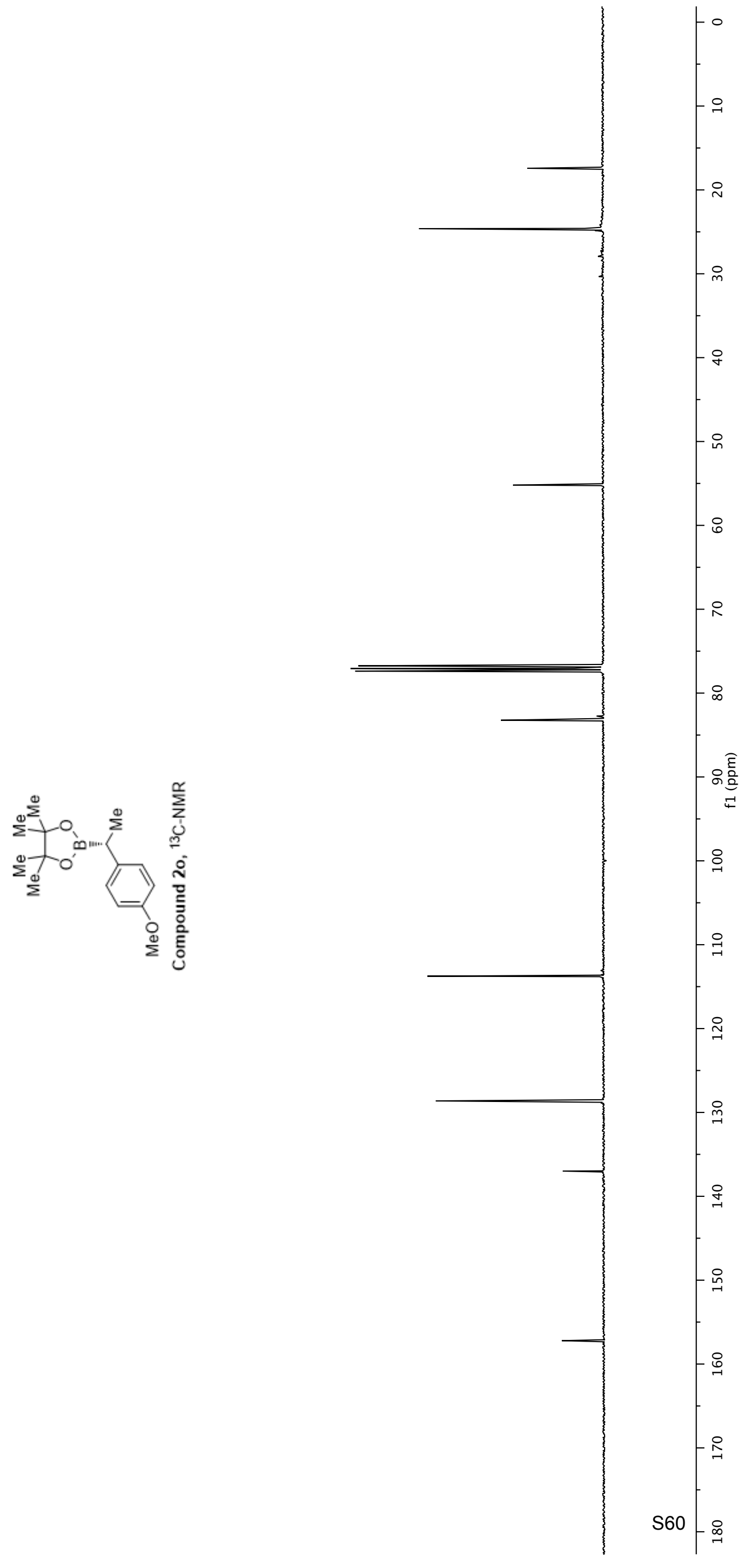

ع9.8ZI -

$66^{\circ} 9 \varepsilon \mathrm{I}-$

IZ $\angle S I-$

:

응

$\infty$

으을

움

욱

$\underset{7}{\stackrel{7}{2}}$

品

昌

운

$\stackrel{8}{\circ}$

4 


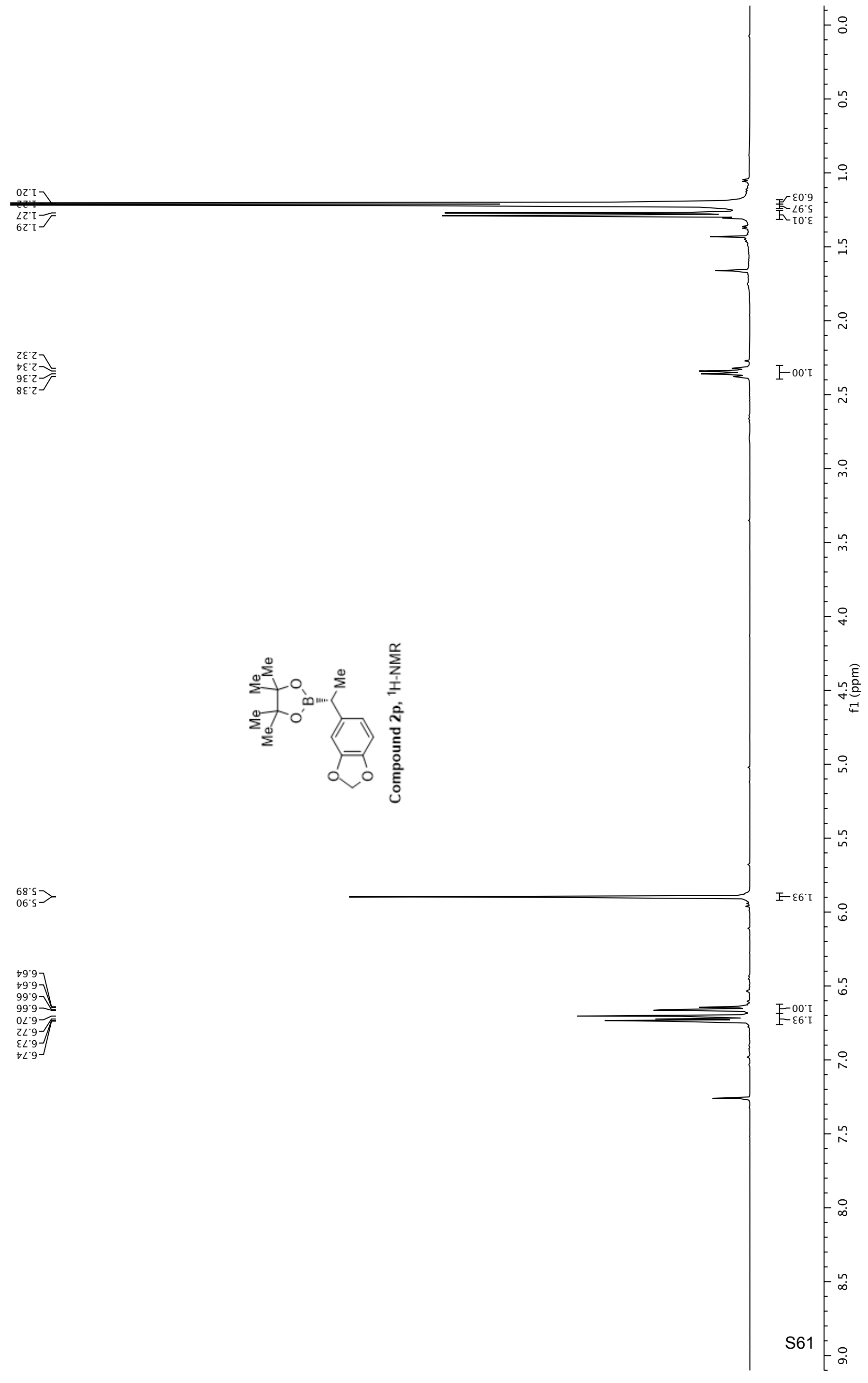




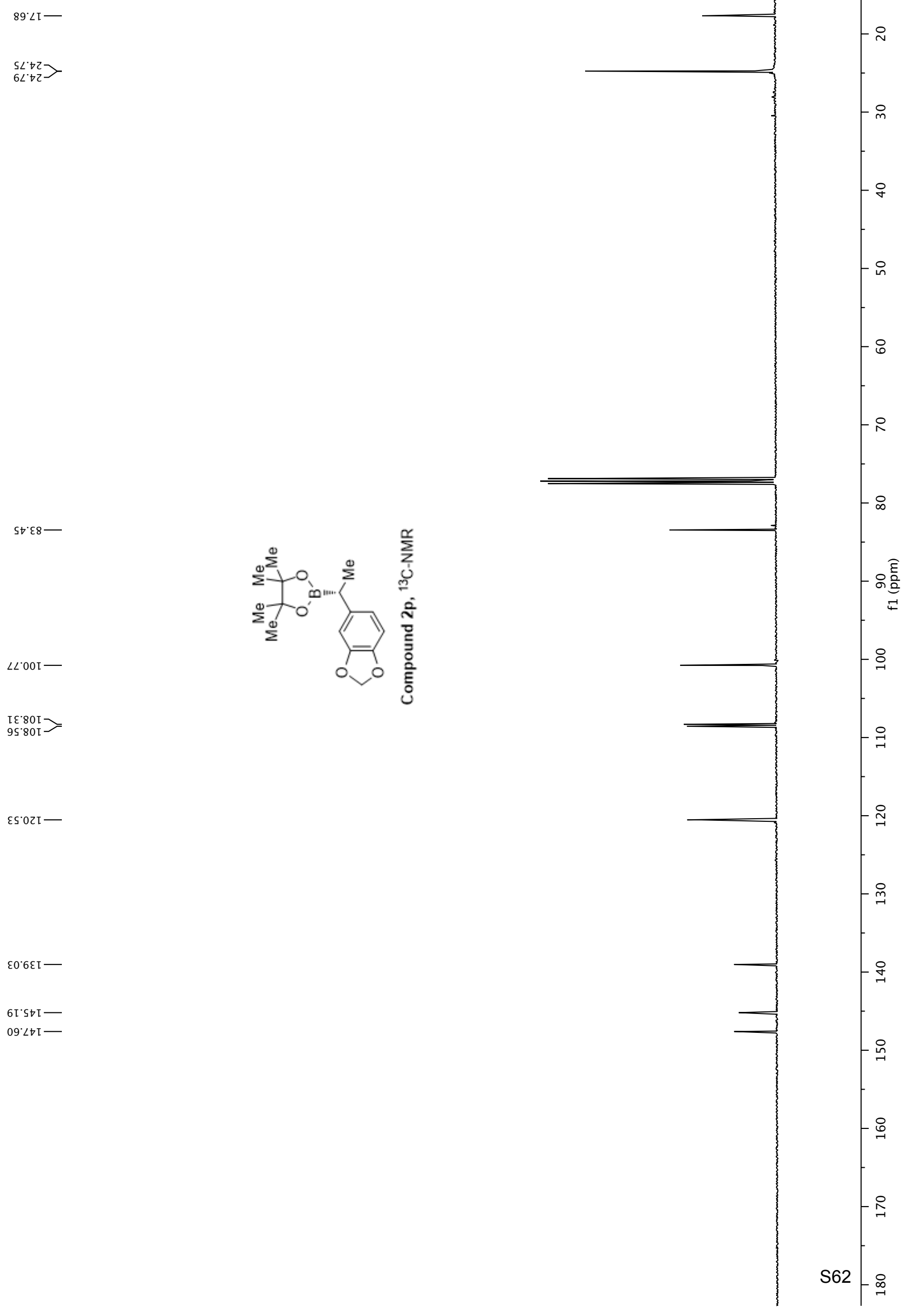




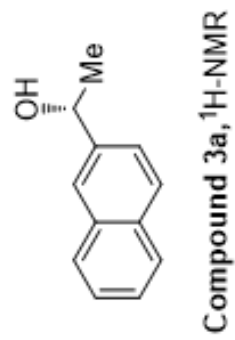




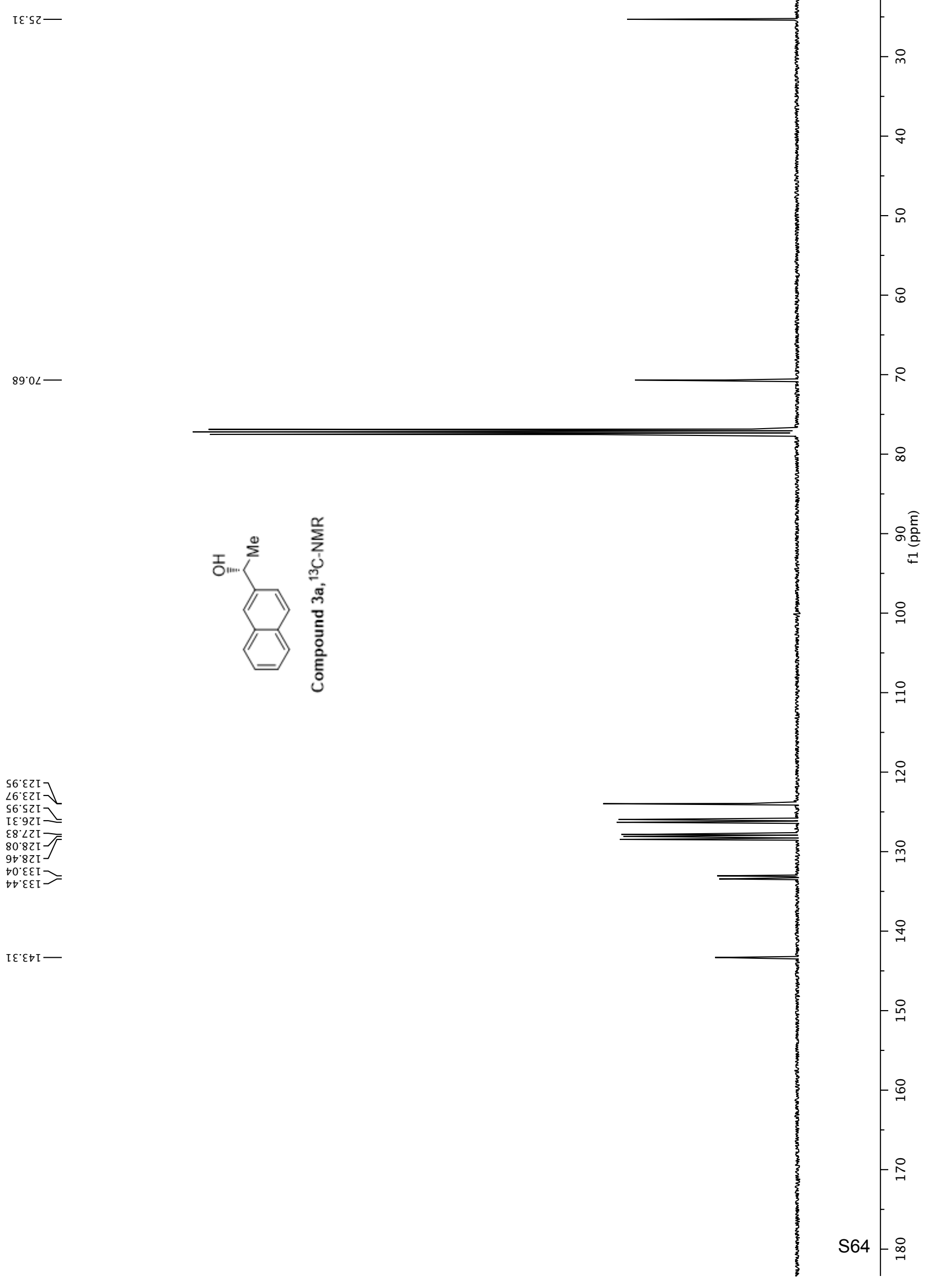


${ }_{69^{\circ} \mathrm{I}} \mathrm{I}=$
$96^{\circ} \mathrm{I}-$

L9.5

89.5
$0<5$

L $\angle$ ' $\mathrm{S} J$

$\left.\begin{array}{l}\angle t^{\circ} \angle \\ 8 t^{\circ} \circ \\ \left.6 t^{\circ} \cdot\right]\end{array}\right]$

$6 t^{\circ}<7$

IS'L]

$\forall S^{\circ}<-$

SS $\angle$ E

$89^{\circ} \mathrm{L}$

89.2

$0<\angle-$

$8 L \angle T$

$88^{\circ}<-5$

$68 . \mathrm{L}$

II. $8>$

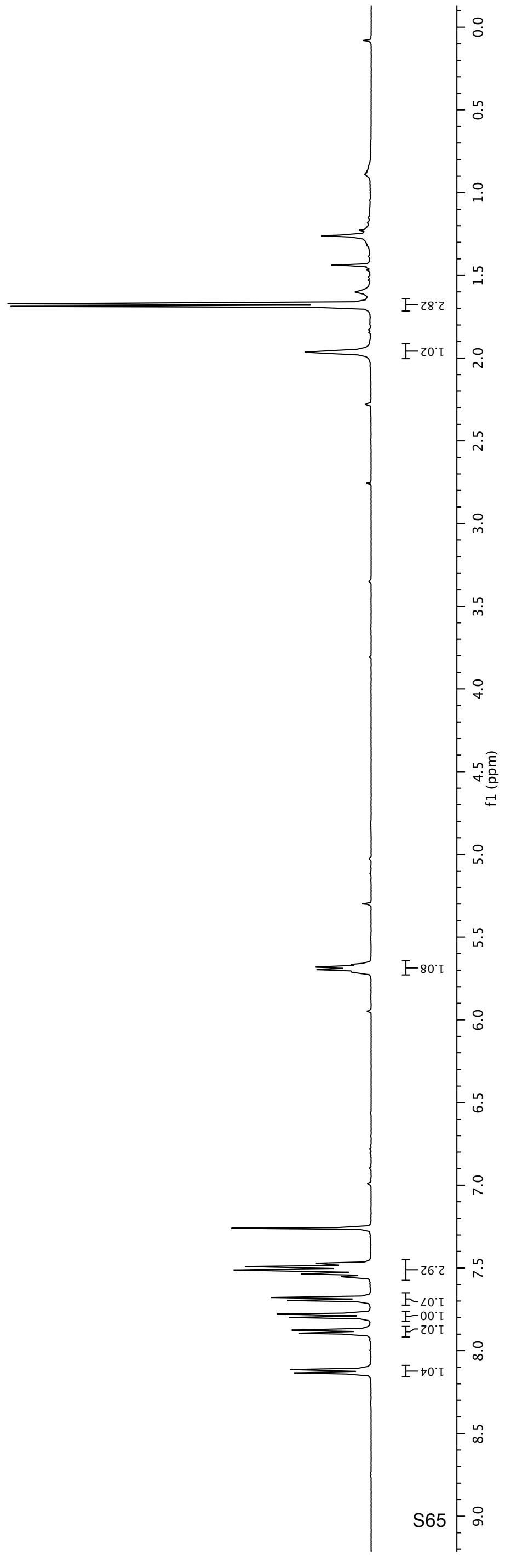

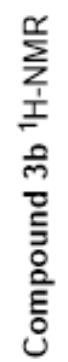


EL'SZI _

2र'92I

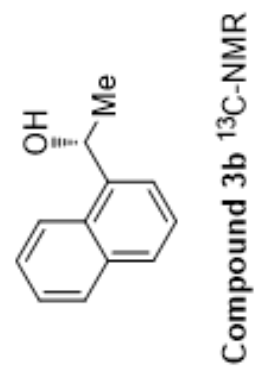

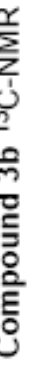


$9 S^{\circ} \mathrm{T}$
$8 \mathrm{~S}^{\circ}$

$\varepsilon 0^{\circ} 乙-$

$26^{\circ} \varepsilon$

[0.5

20.5

เo.s

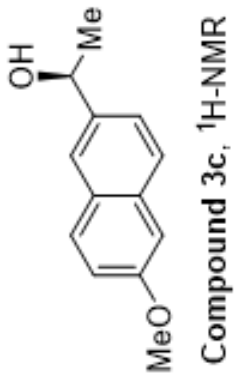

$\varepsilon \tau^{\circ}\llcorner]$

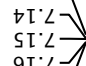

9I' $\angle J$

$9 \sigma^{\circ}<>$

$80^{\circ}<>$

I $\angle L \perp$

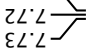

然

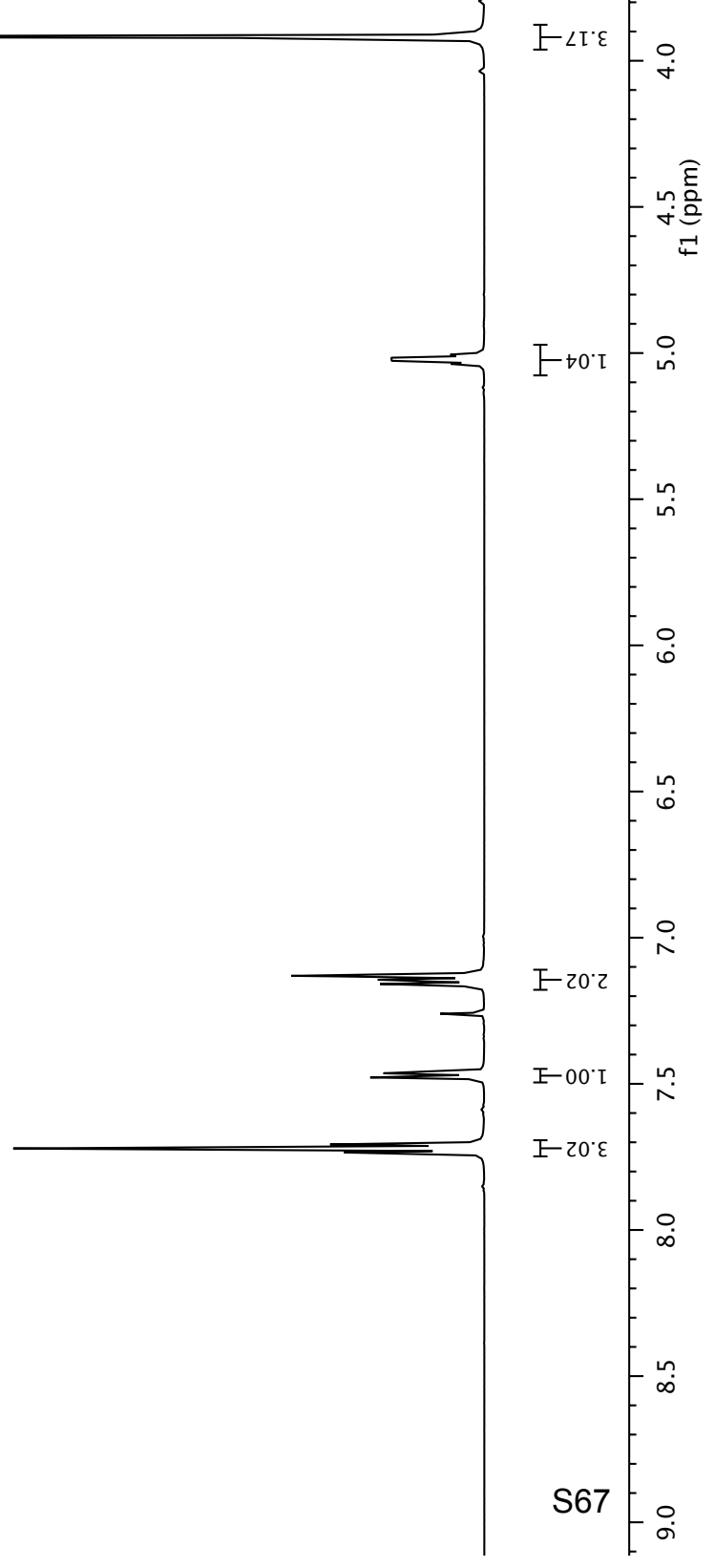



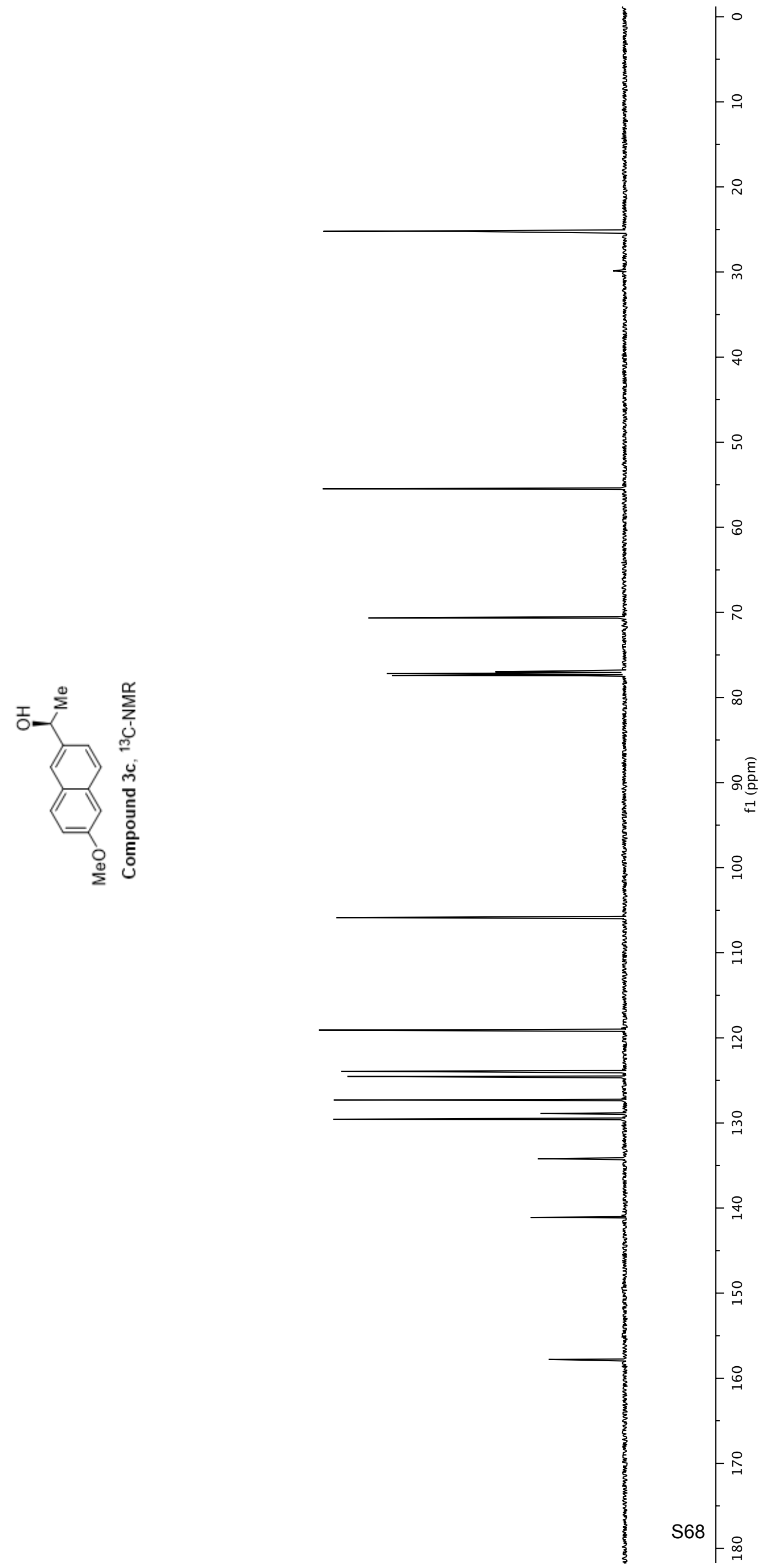

$60 \%$ I I

$26{ }^{\circ} \varepsilon 2 \mathrm{I}-$

$\varepsilon S^{\circ} \sqcup Z \mathrm{LI}$

$06.82 \mathrm{I}$

$95.62 \mathrm{I}=$

$0 Z^{\circ} \sqcup \varepsilon I$

$0 I^{\circ} \mathrm{It} I$

$6 L \cdot \angle S I-$ 


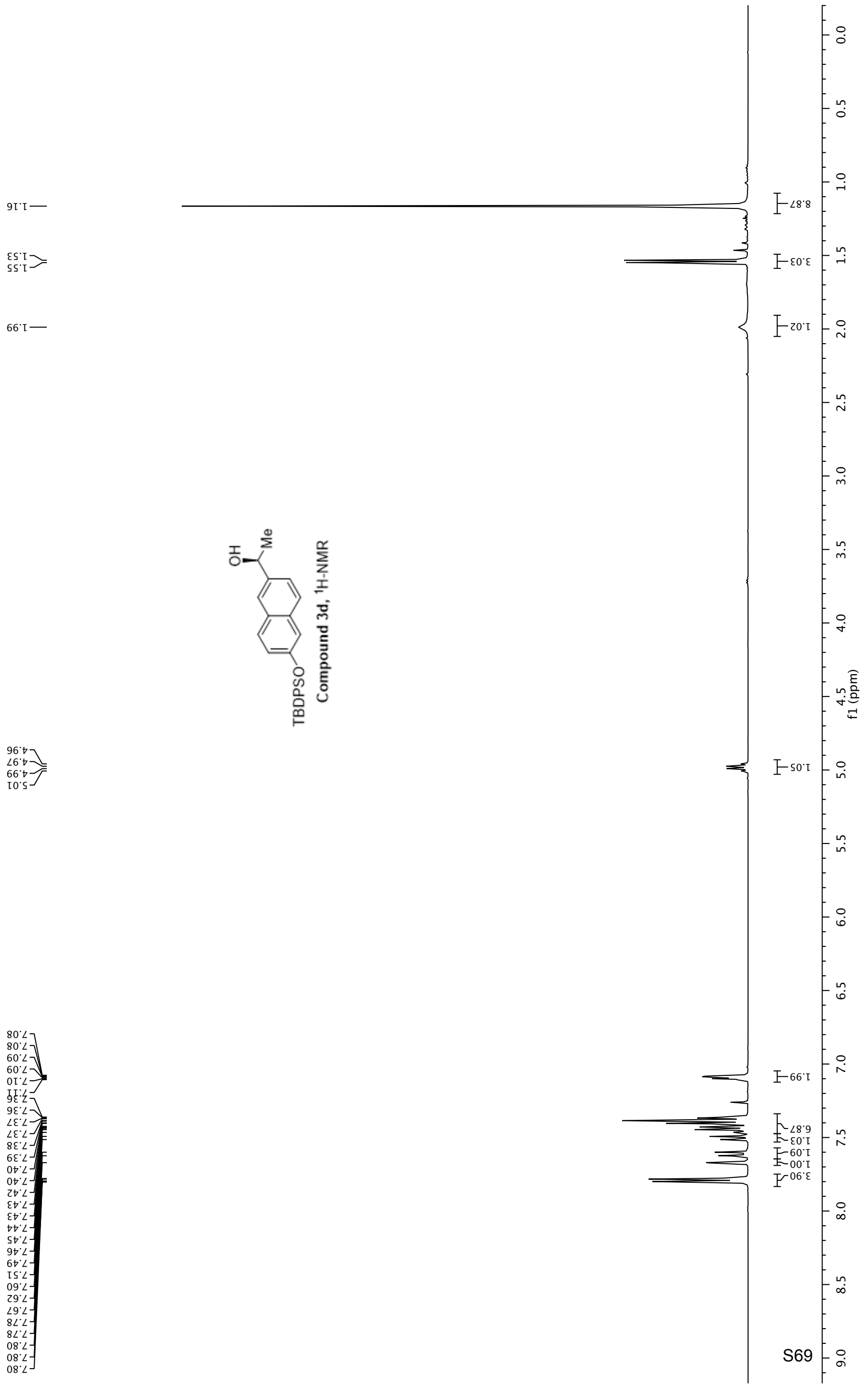




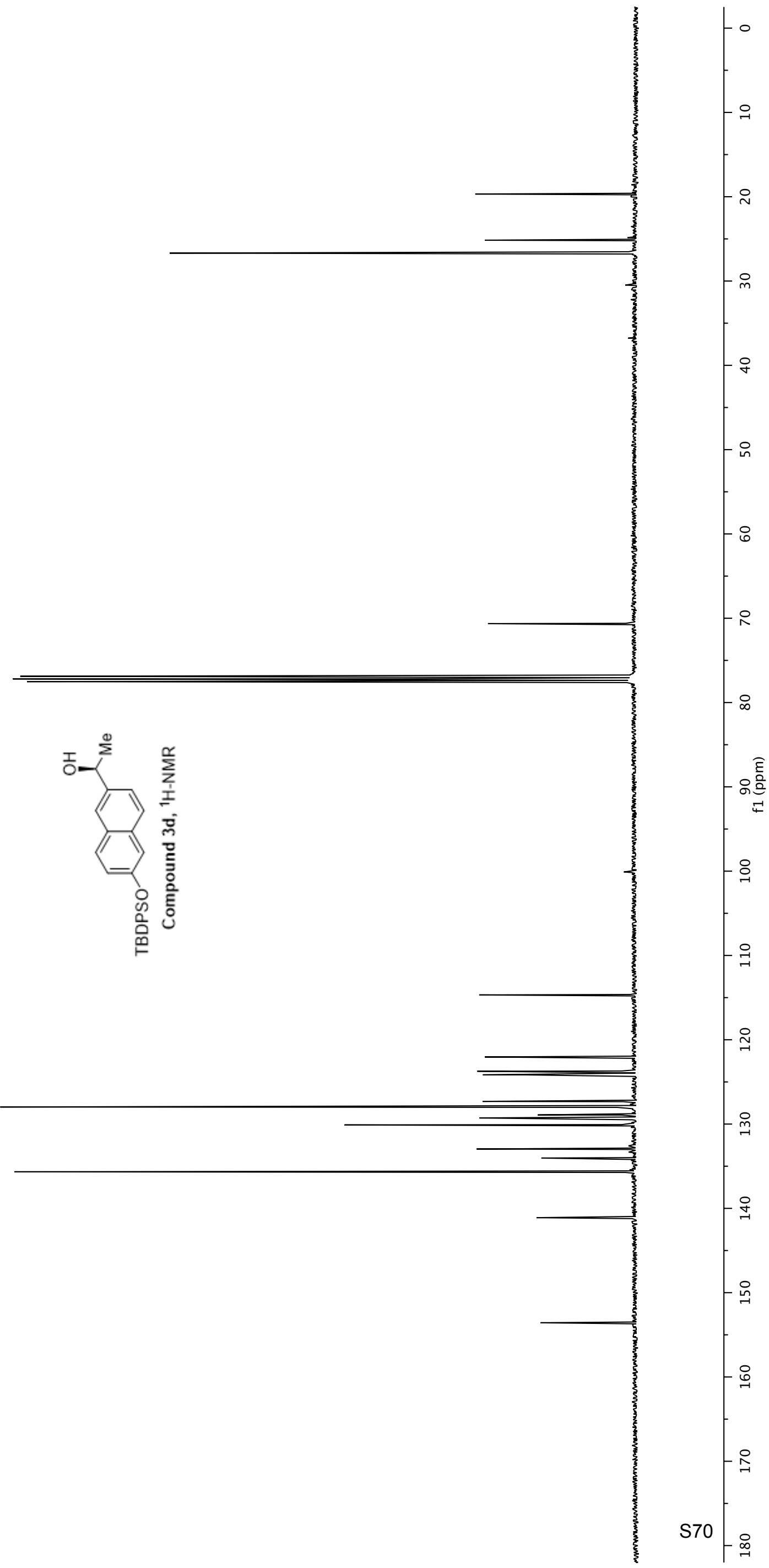

$\rightarrow 0.221-$

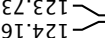

乙E' $\angle Z I \backslash$

$86 . \angle Z I-$

$26.82 \mathrm{I}-\bar{T}$

$8 \mathrm{Z}^{\circ} 6 \mathrm{LI}-$

0โ

घ०. $\bullet \varepsilon I-$

99. SEI

I I ' $I t$ I

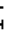

옹

$\angle S^{\circ} \varepsilon S I-$ 


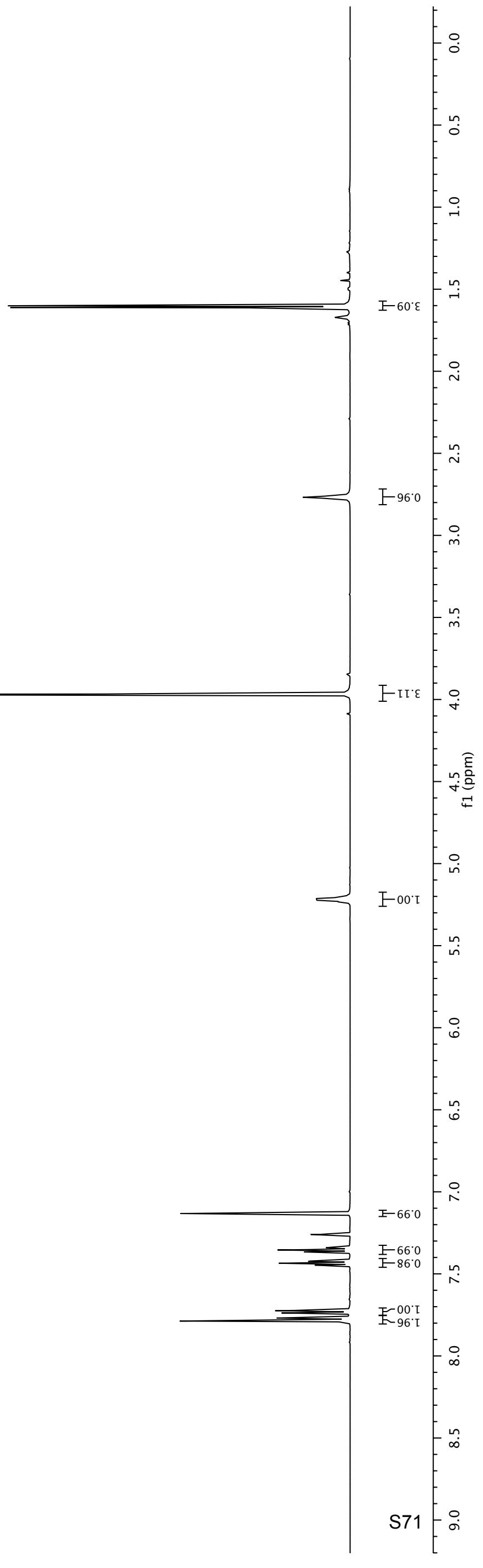

$\left.\begin{array}{l}0 Z \cdot S \\ \text { IZ' } \mathrm{S} \\ 2 Z \cdot \mathrm{c}\end{array}\right]$

2z.5

$\angle 6^{\circ} \varepsilon$

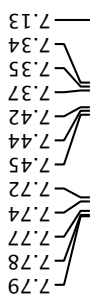

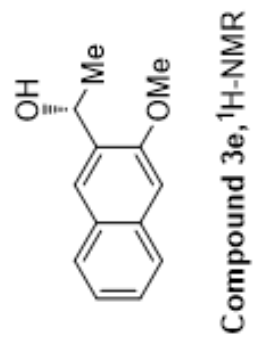

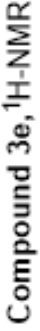

हิํㅡㄹ 


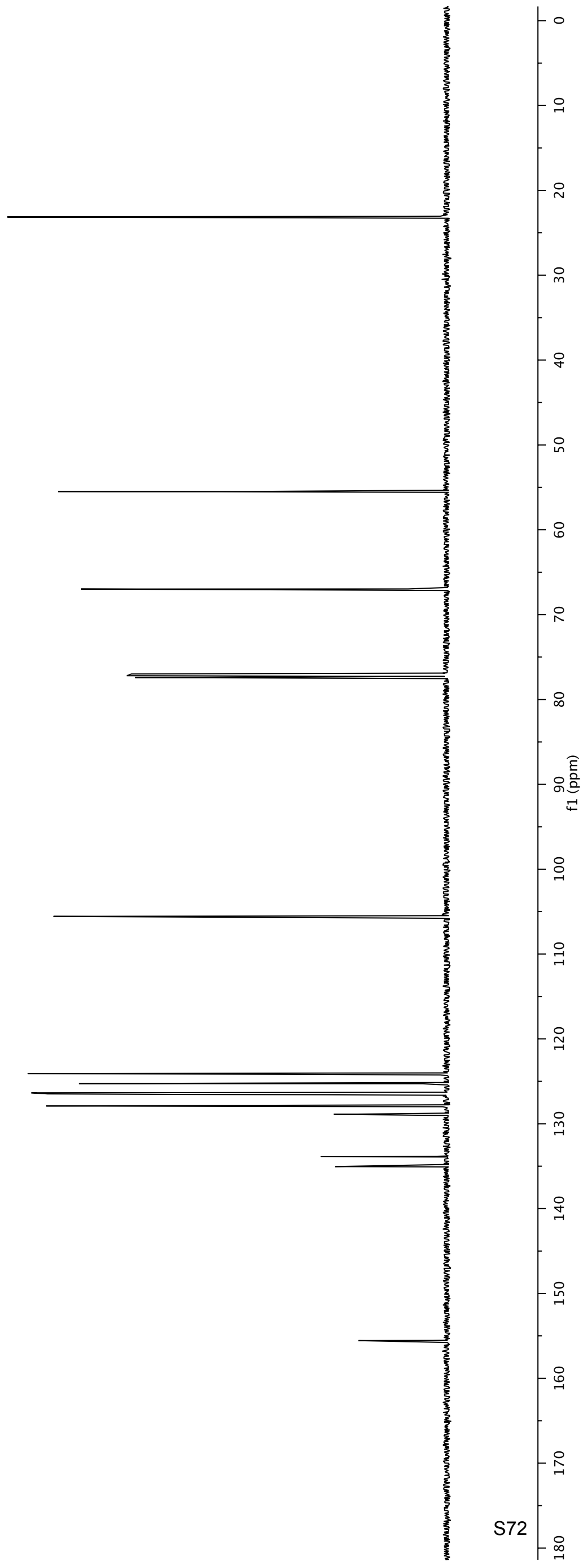

$\angle 0^{\circ} \circ Z I$

SE.9ZI

9S. $50 \mathrm{I}$ 
ES.
SS.

$26^{\circ} \mathrm{L}$
E6

$86^{\circ} \mathrm{t}$

$20^{\circ} \mathrm{s}$

52.97

$9<\cdot 9>$

$9<\cdot 9$

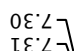

$\varepsilon \varepsilon \cdot L]$

$\varepsilon \varepsilon<-$

$\angle t^{\circ} L>$

$\left[9^{\circ}<>\right.$

[9.<

$\varepsilon 9^{\circ} \angle-$

$\varepsilon 9 .<$

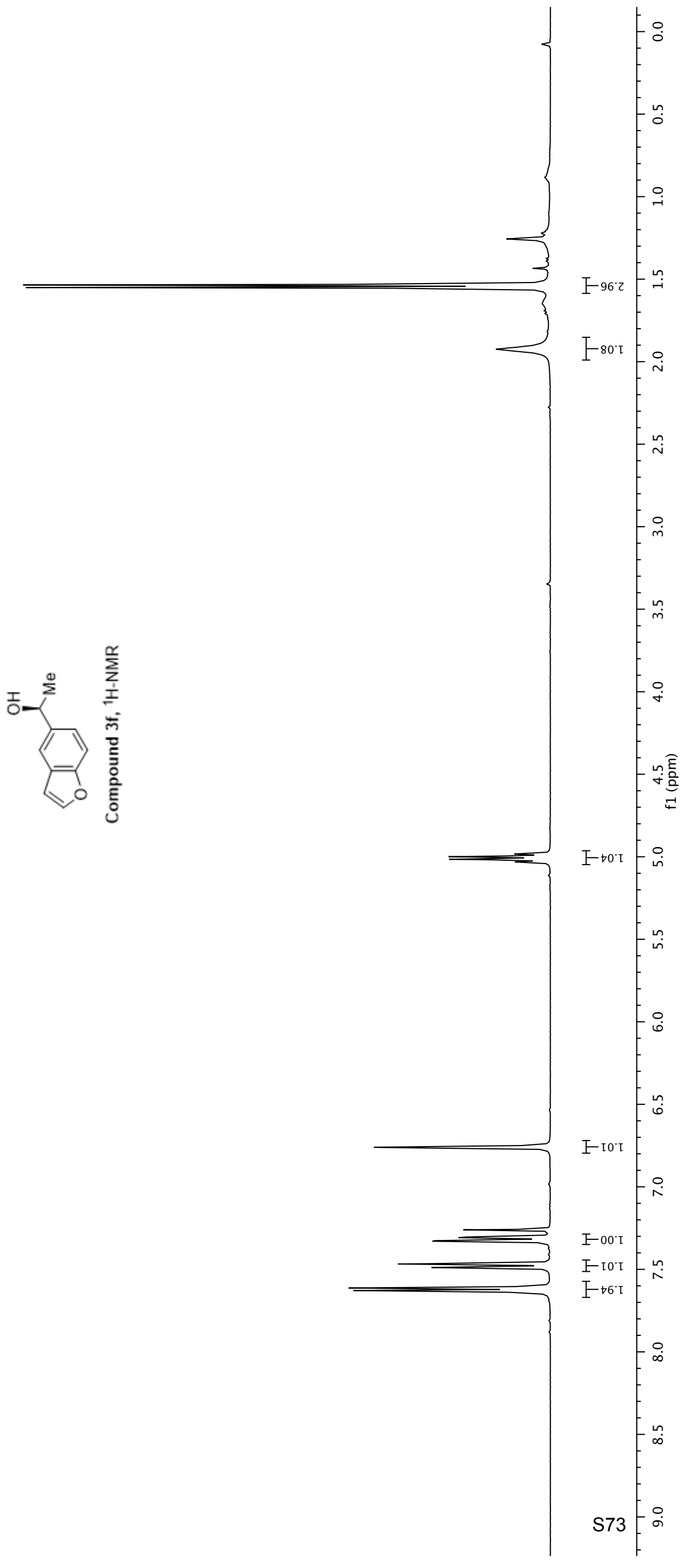




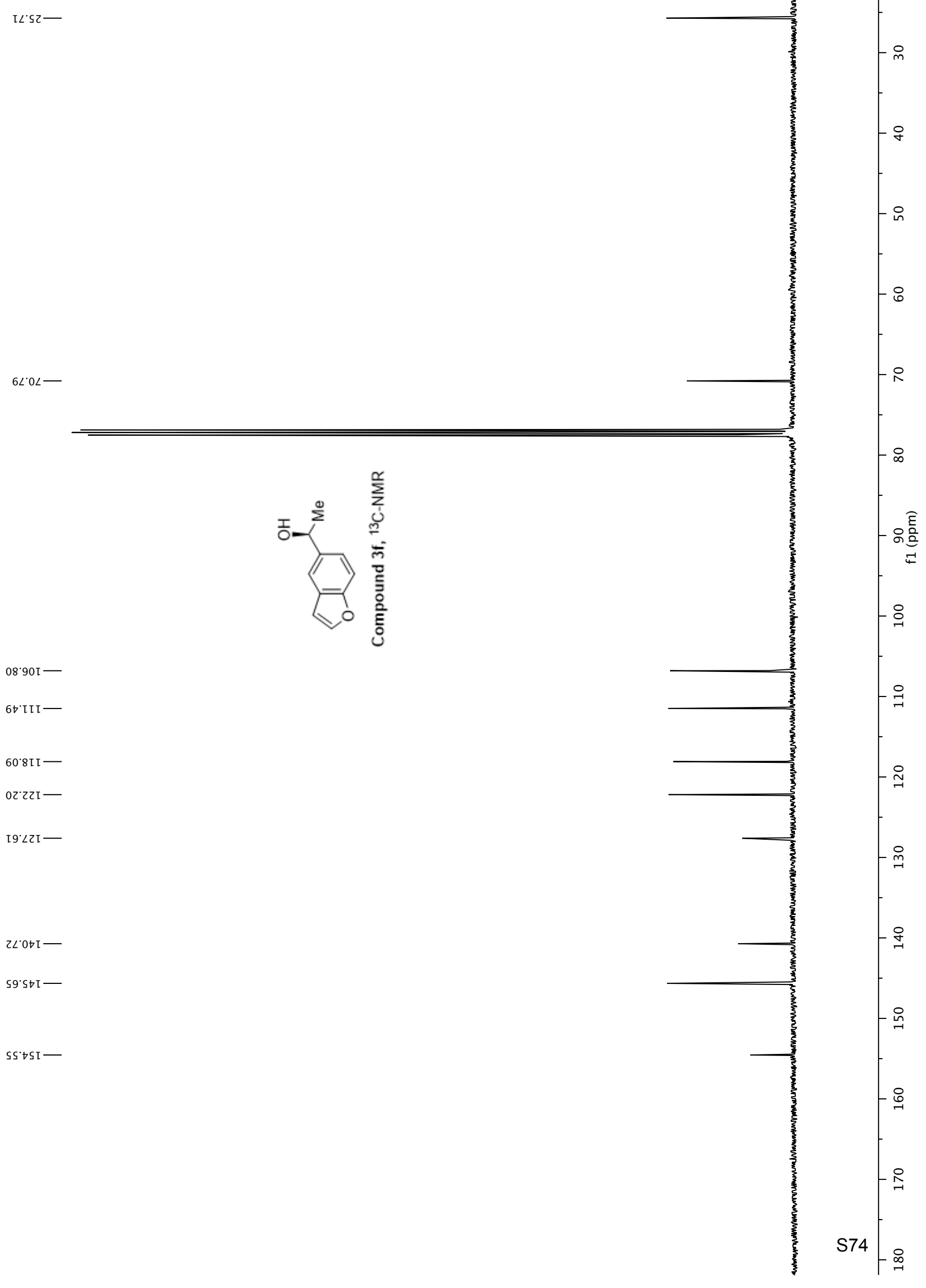




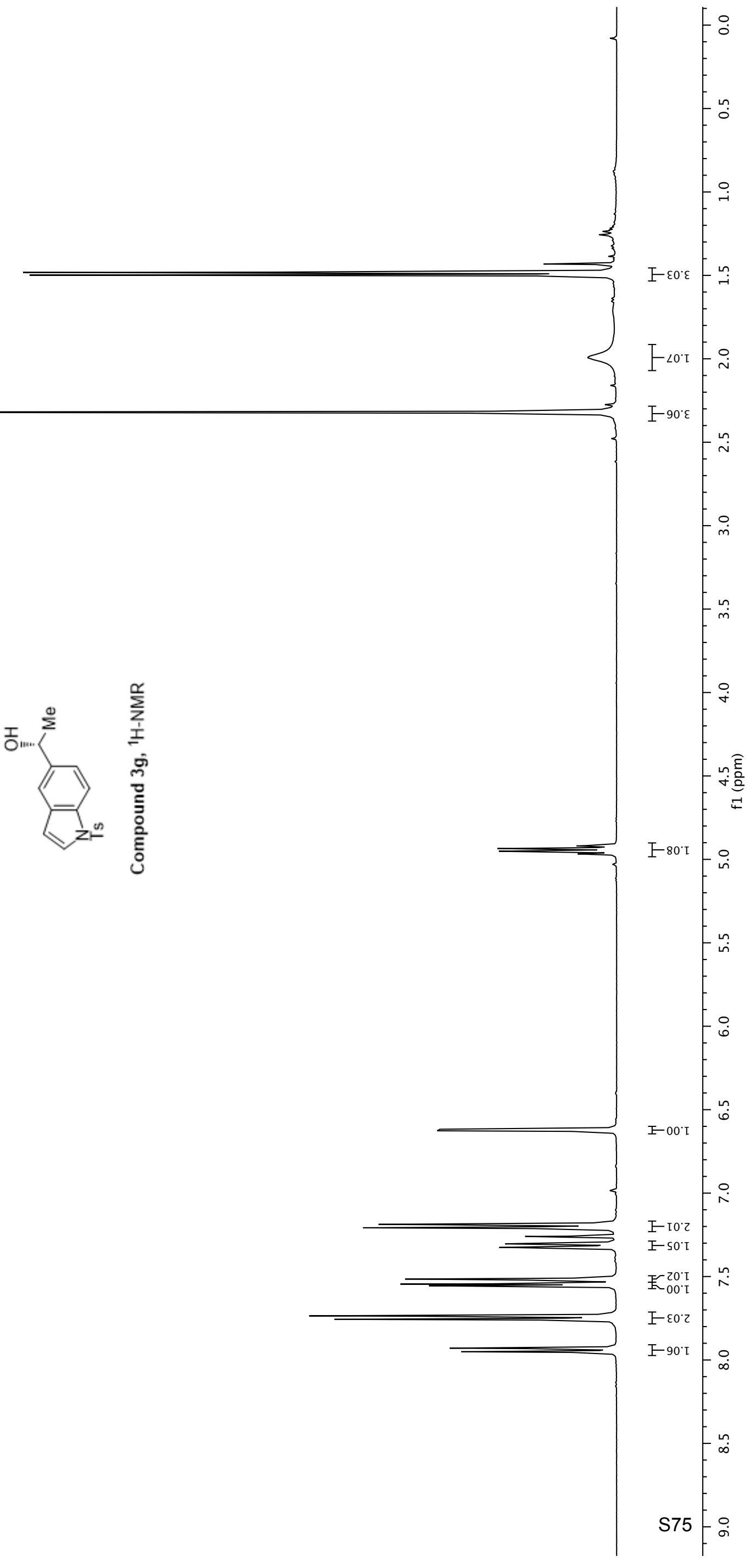




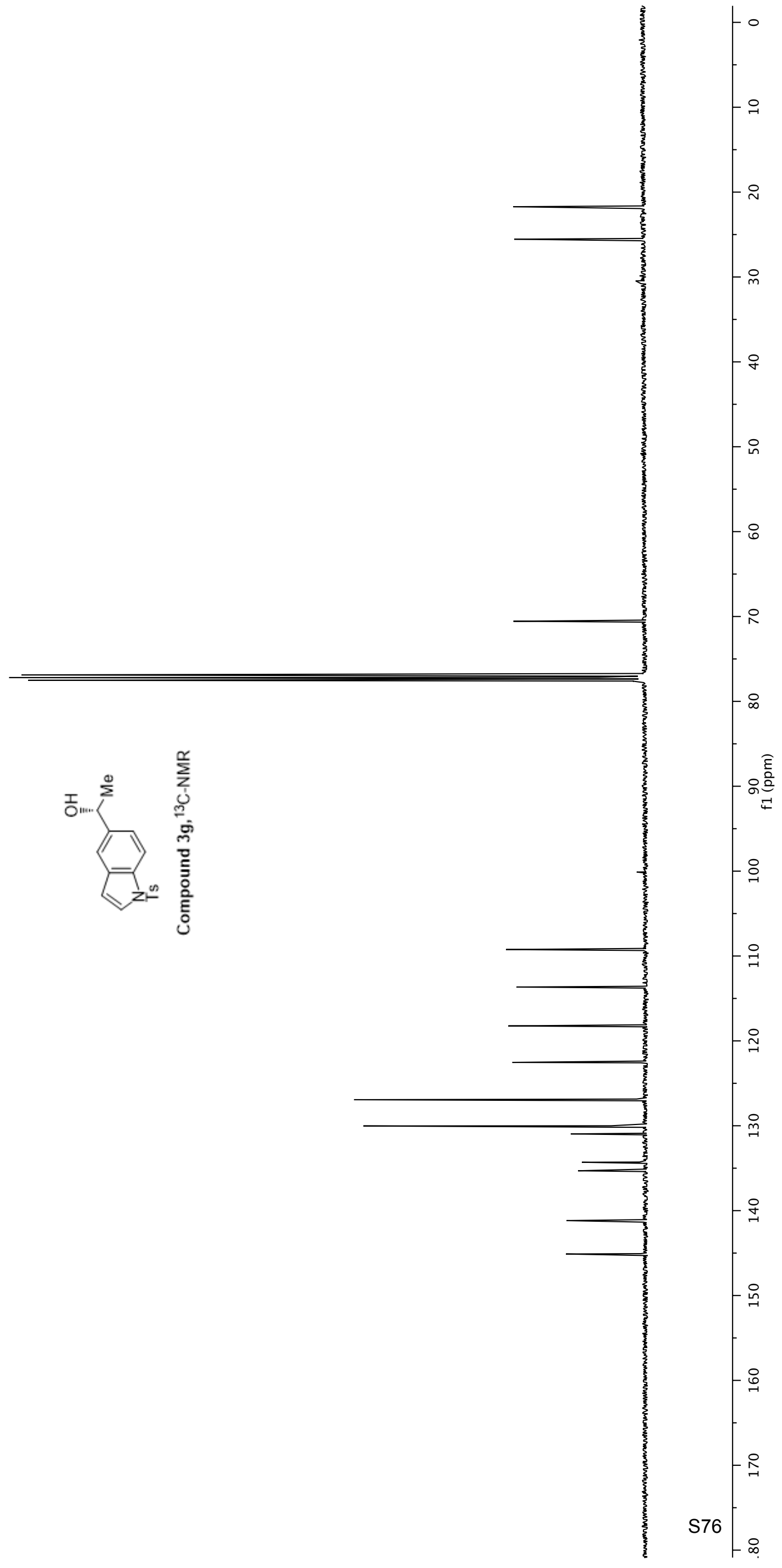


โ8.

$88^{\circ} \circ$

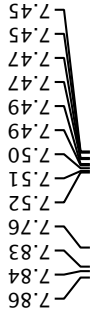

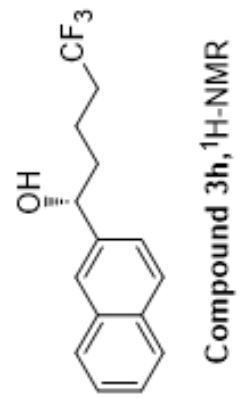

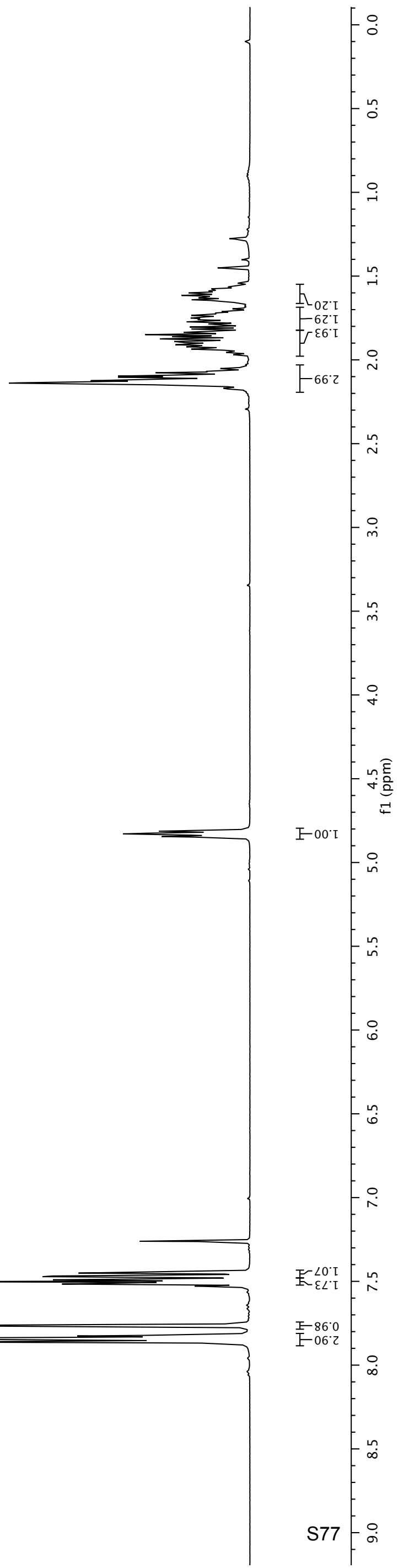


$09.8 \mathrm{I}$
$\varepsilon 9.8 \mathrm{I}$

99.8 $\mathrm{I}$

$69.8 \mathrm{I}$

โ $\varepsilon \cdot \varepsilon \varepsilon]$

$6 S^{\circ} \varepsilon \varepsilon-$

$88^{\circ} \varepsilon \varepsilon-$

$9 \mathrm{I}^{\circ} \downarrow \varepsilon$
I8 $\angle \varepsilon$

ZI' $\varepsilon Z[-$

$\varepsilon 6^{\circ} \varepsilon Z[]$

$\angle 8 . S 2[]$

$8 \mathrm{I}^{\circ} 9 \mathrm{~L} \mathrm{~L}-$

80.251

$88^{\circ} \angle 2 \mathrm{~L}$ T

$80.82 \mathrm{I}-$

89.82[

$9 \varepsilon$ โ ¿ [

OZ'દદ -

$\angle 9^{\circ} \mathrm{I} t \mathrm{I}$

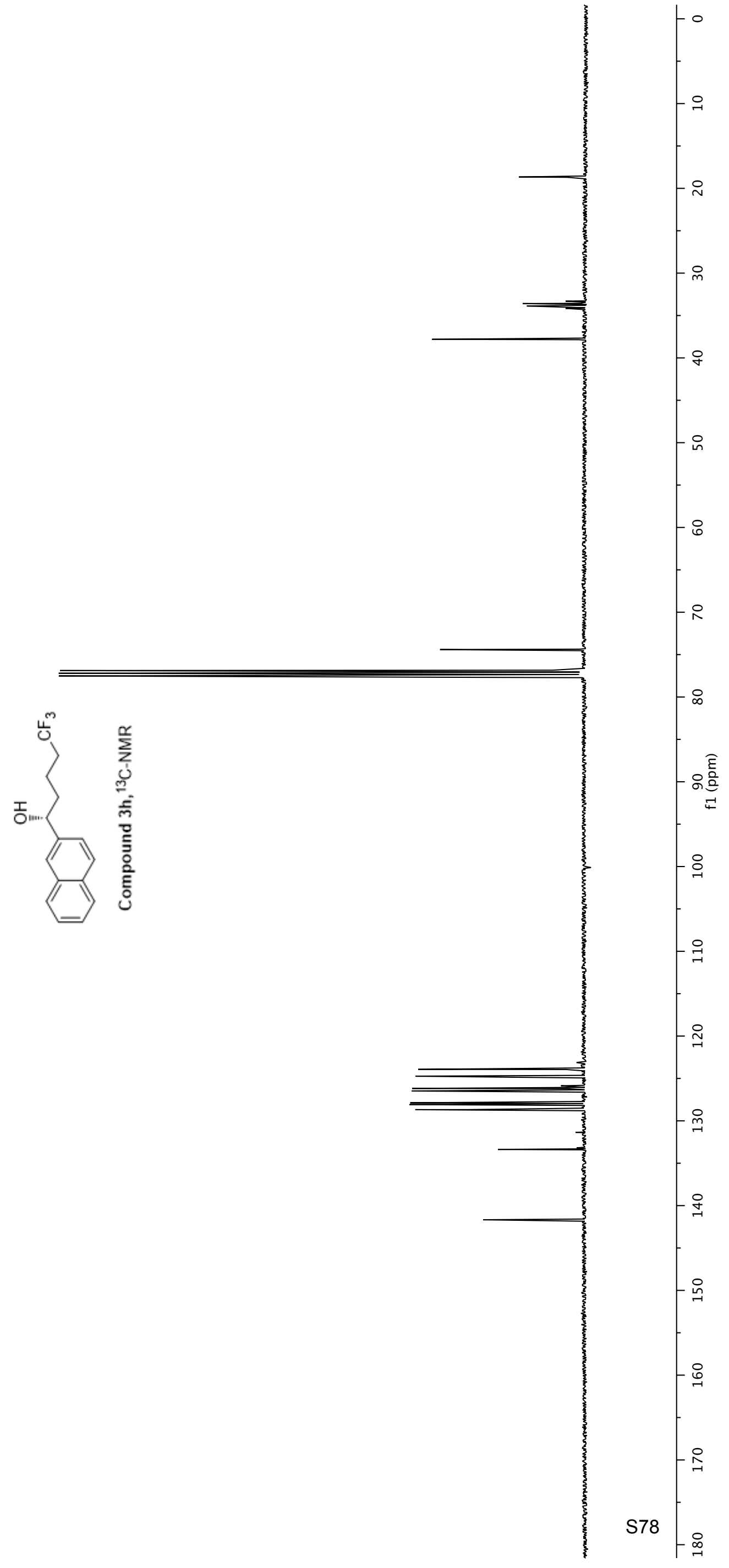




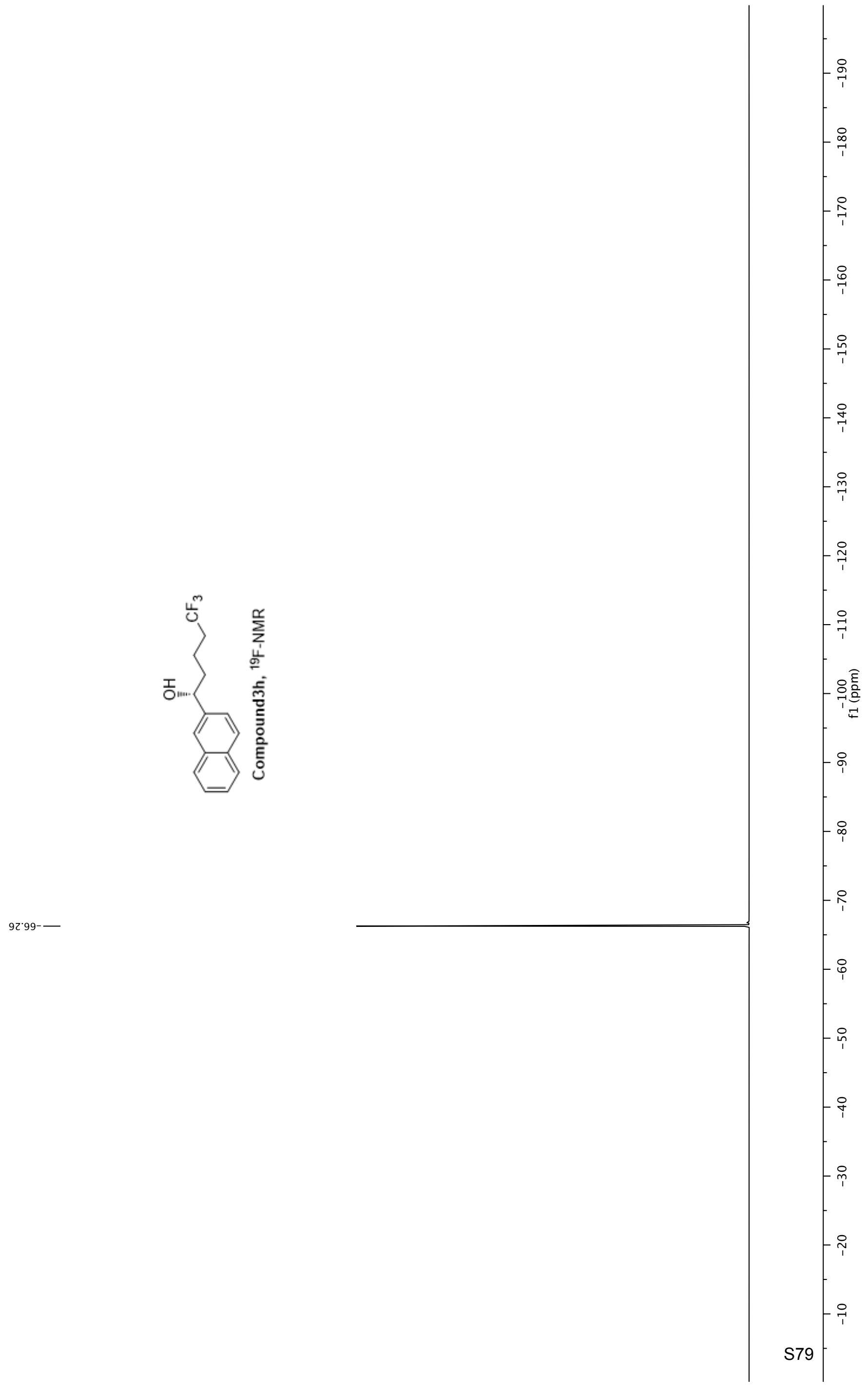




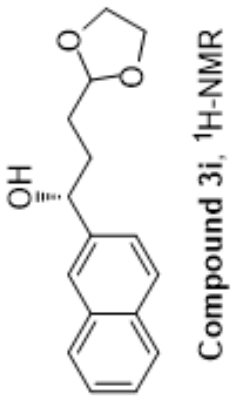




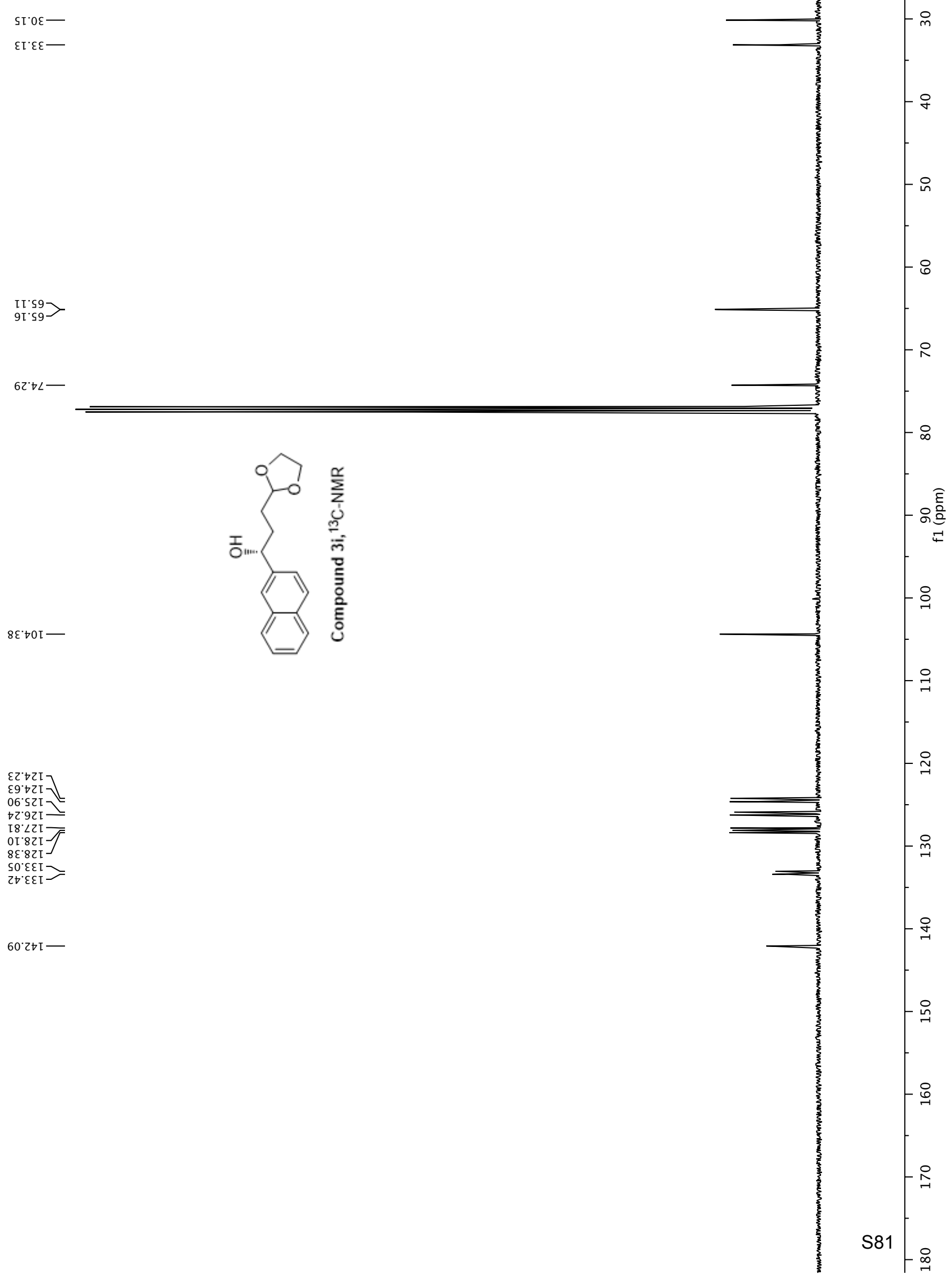



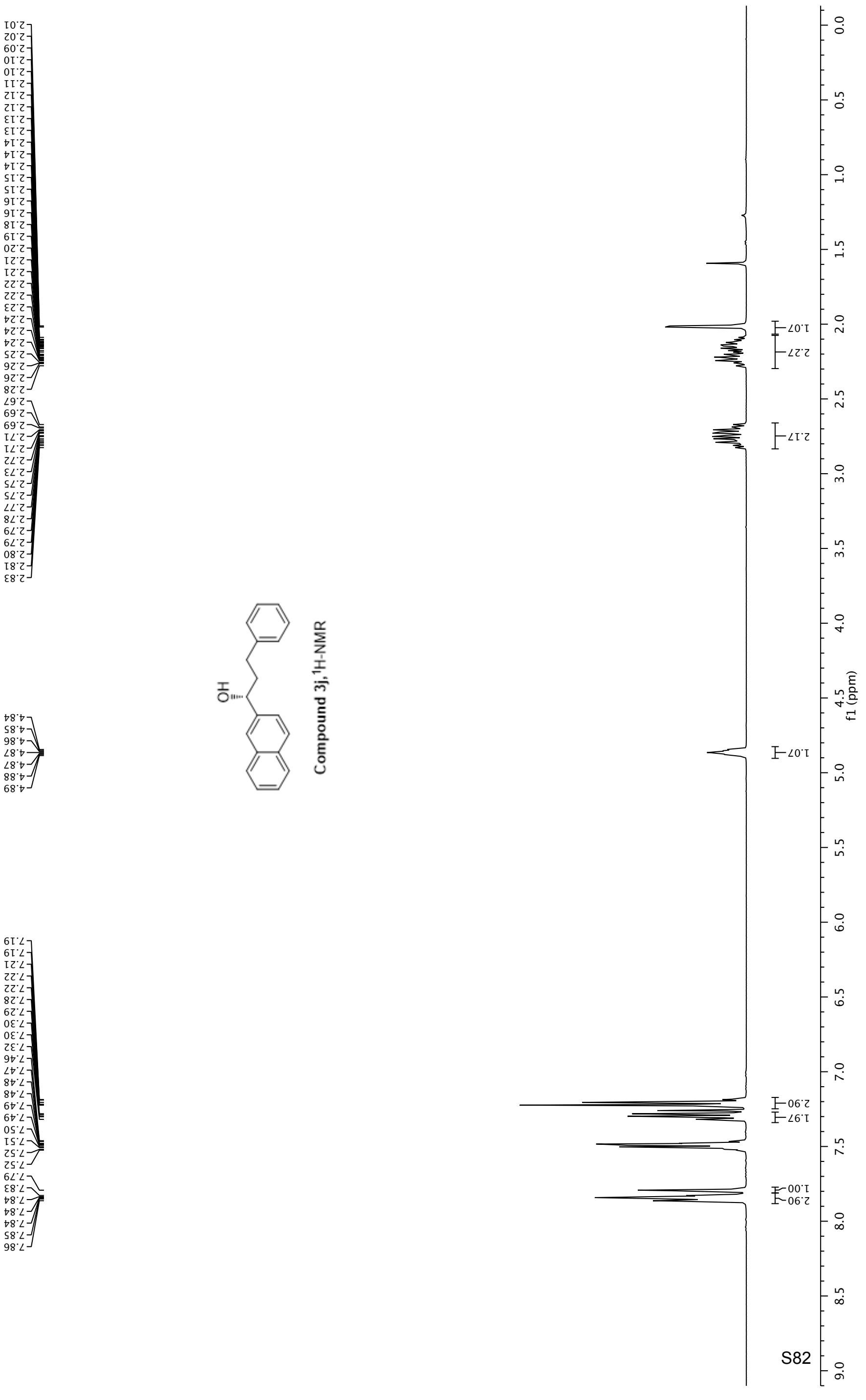

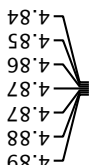




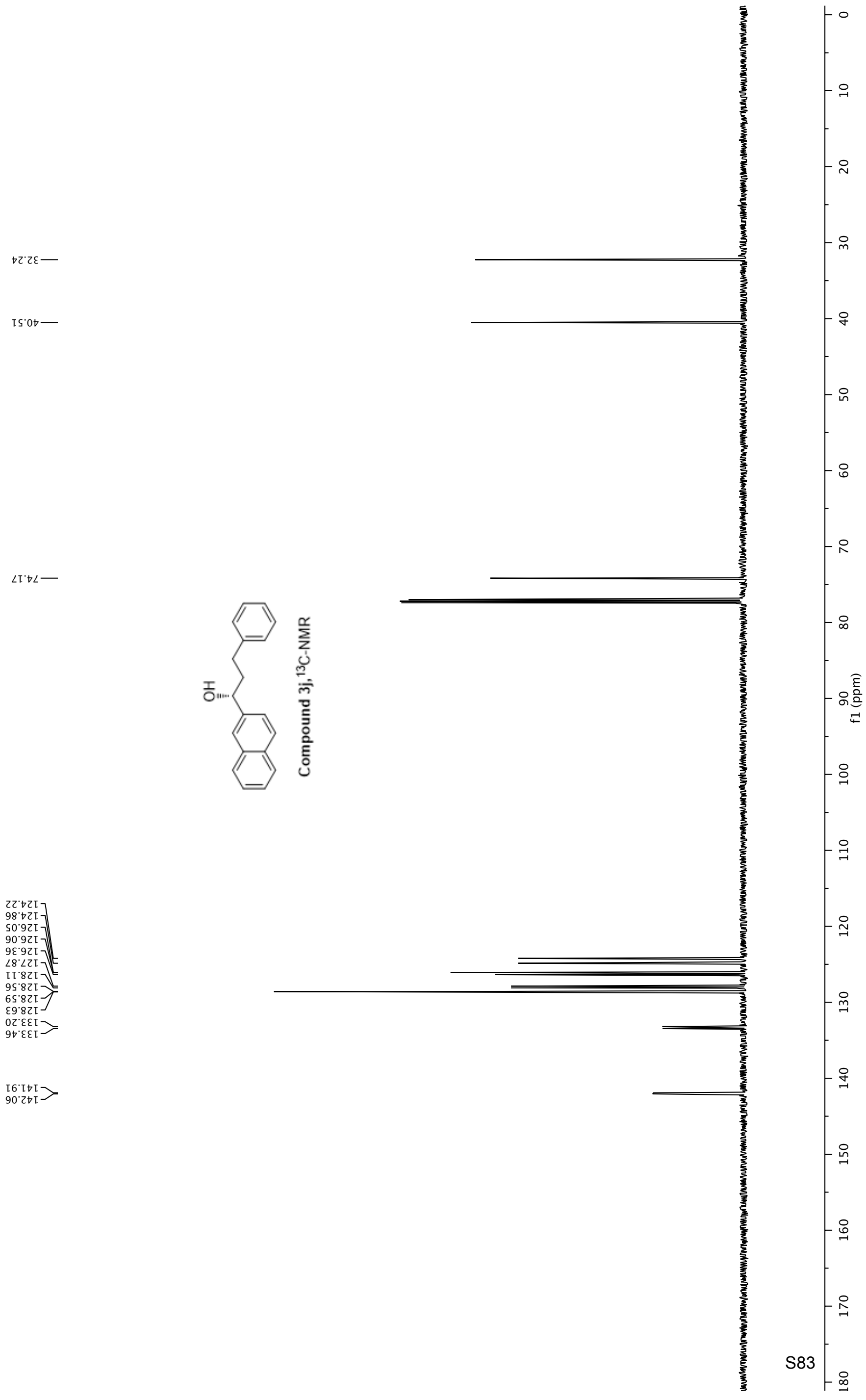


78.0

$58.0-$

$\rightarrow 0^{\circ} \mathrm{I}$

SO $\mathrm{T}$

$\left.\begin{array}{l}\varepsilon 6^{\circ}[ \\ \forall 0^{\circ} 2 \\ 50^{\circ} \cdot\end{array}\right]$

$50.2]$

$\left.\begin{array}{l}90^{\circ} \mathrm{Z} \\ \angle 0.2\end{array}\right]$

80.2

0 ' $2-$

2I' 2 I'

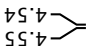

$9 t^{\circ} L$

$\left.9 t^{\circ}<\right]$

$9 t^{\circ}<$

$\angle t \circ]$

$80^{\circ}<$

$6 \sigma^{\circ}<$
$0 S$

$9 L \angle$

28.27

88.

$\forall 8^{\circ}<-1$
$\forall 8 . \angle$

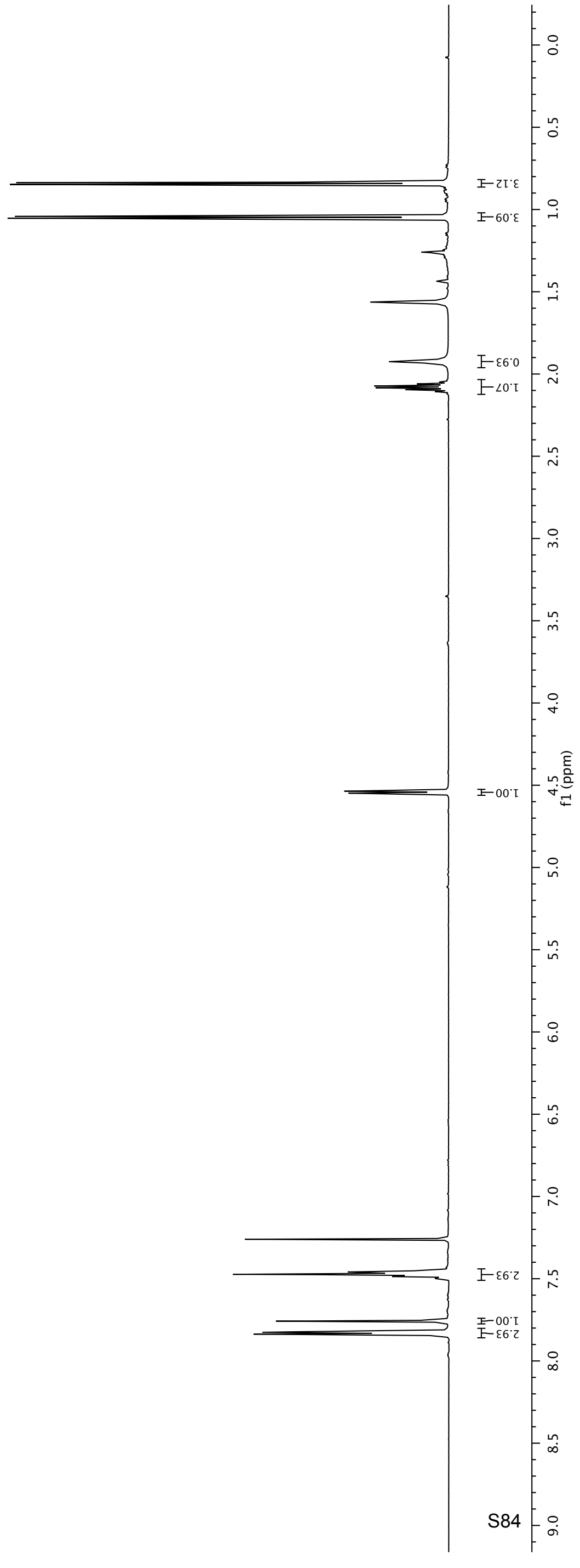

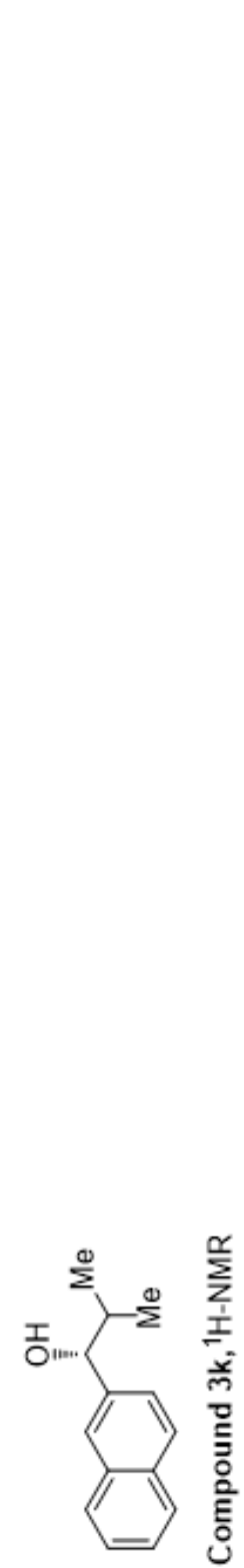

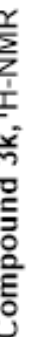


$t{ }^{\circ} 8 \mathrm{I}=$
$\varepsilon \varepsilon \cdot 6 \mathrm{I}$

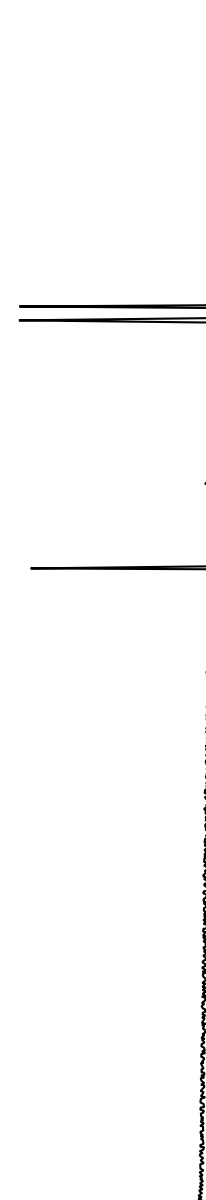

$\angle \varepsilon \cdot \varsigma \varepsilon$

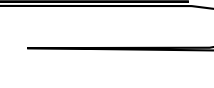

$-\infty$

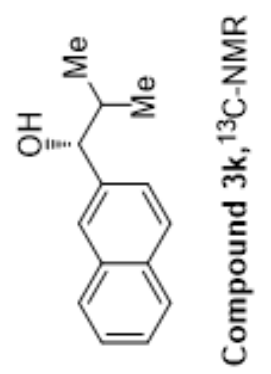

으을

ع8 221-

60.82[

ОI. $દ \varepsilon[-$

૦દ'દદા

$S Z^{\prime} I t I-$

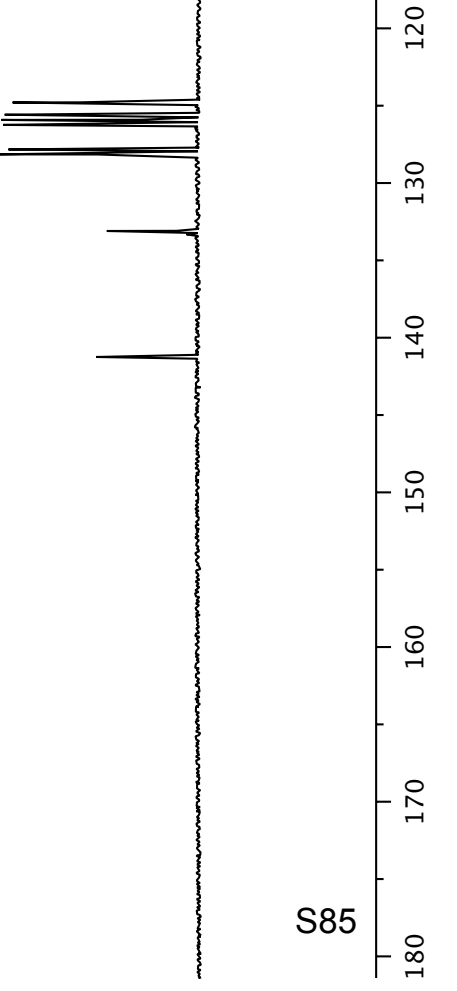


$\angle t \cdot T$
$8 D^{\circ} I$

$\angle 8^{\circ} \mathrm{T}$

$\angle 8^{\circ} \circ$
$88^{\circ} \circ$

$88^{\circ} t$

06.5

ᄂ0. 2

†0 $\mathrm{L}$

$\varepsilon \varepsilon^{\circ}\llcorner]$

$\neg \varepsilon^{\circ}\llcorner$

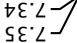

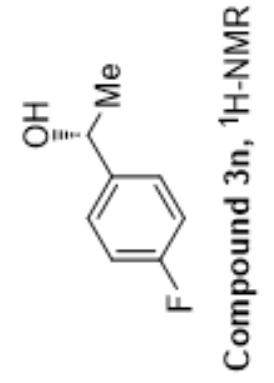

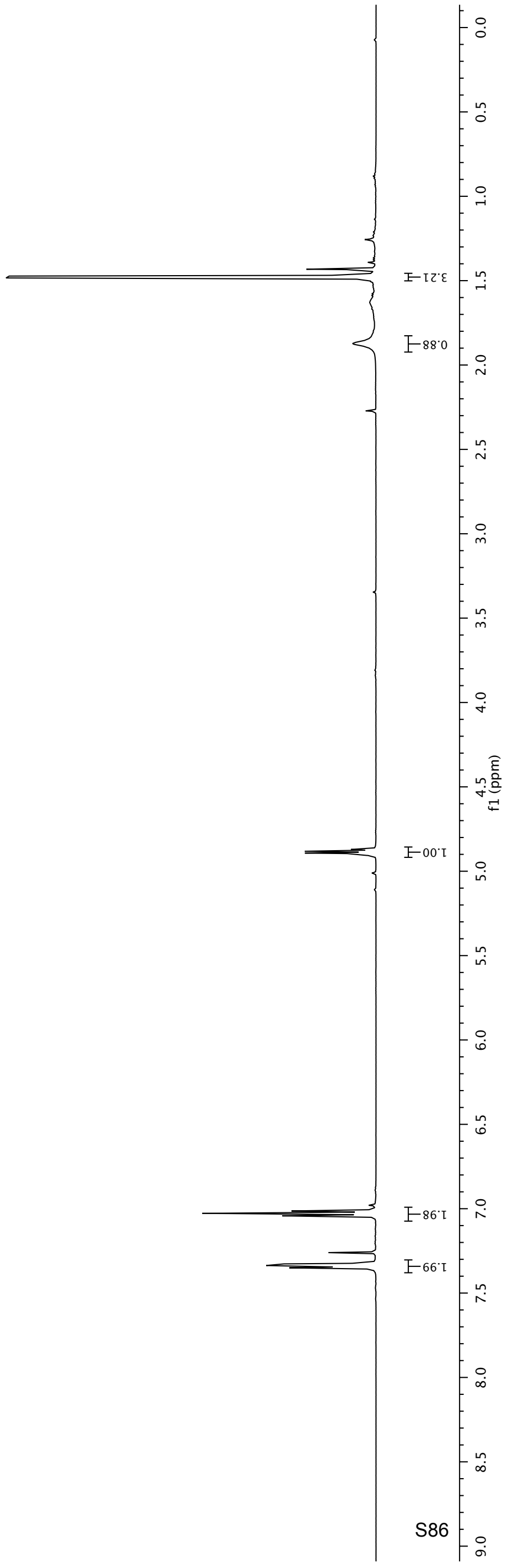


$8)^{\circ} s$

$96.69-$

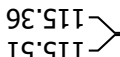

$\left.\begin{array}{l}6 I^{\circ} \angle Z I \\ b Z \cdot L Z I\end{array}\right\rangle$

$0 L^{\prime} T \sqcup I-$
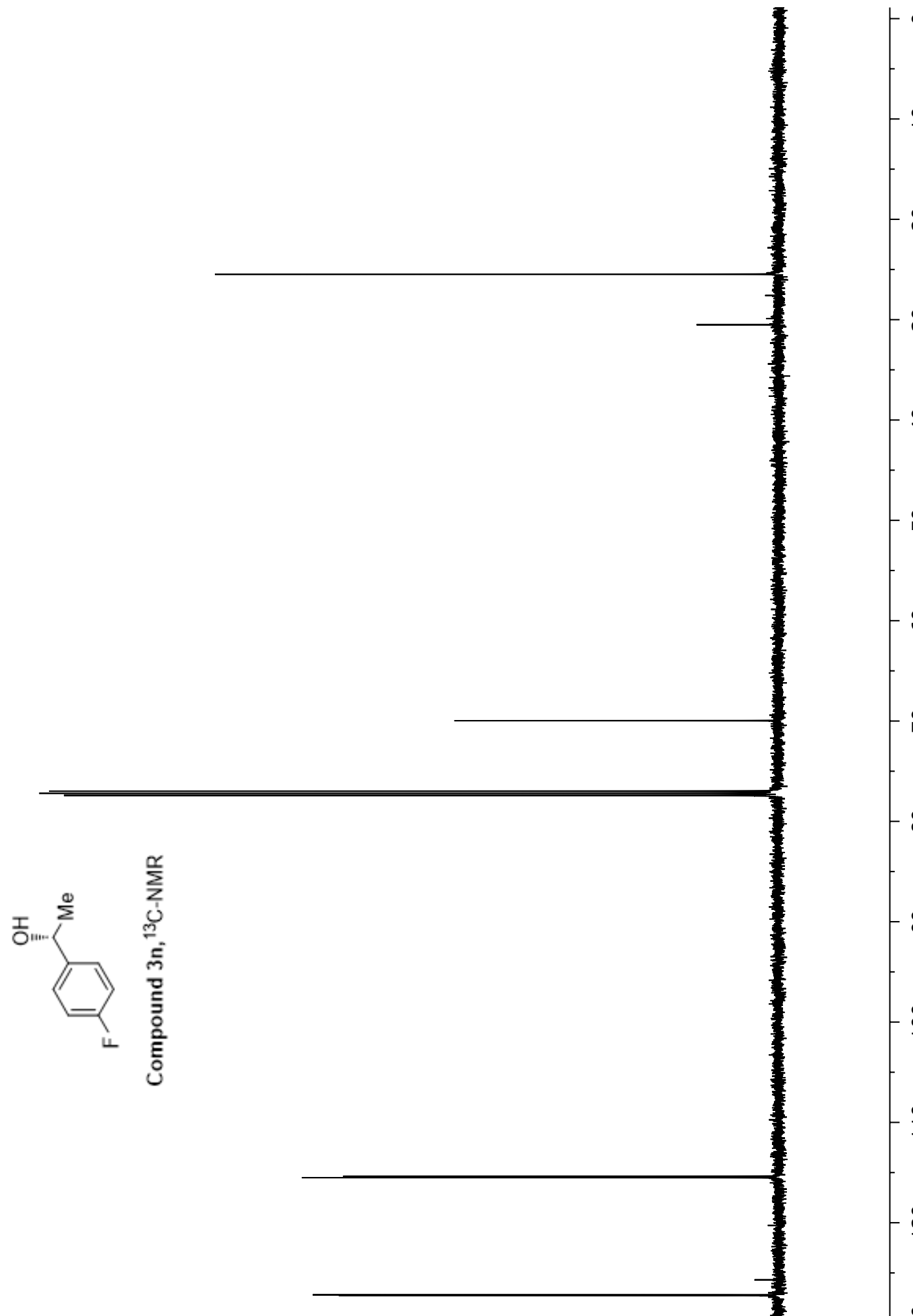

음

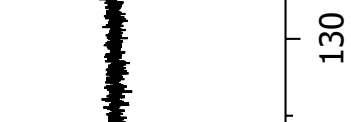

움
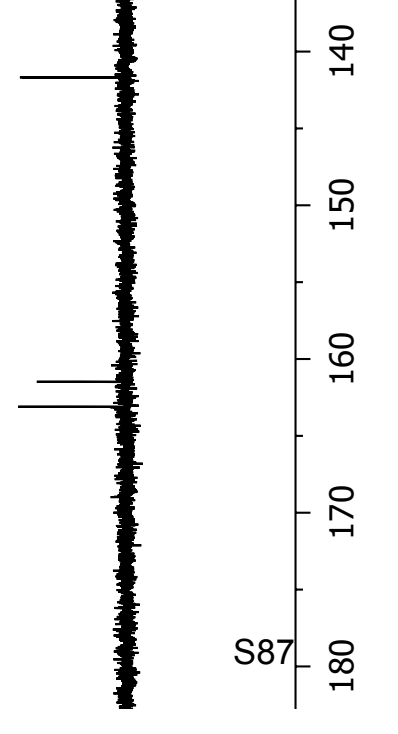

86. T9I-

ОI' $\varepsilon 95-$ 


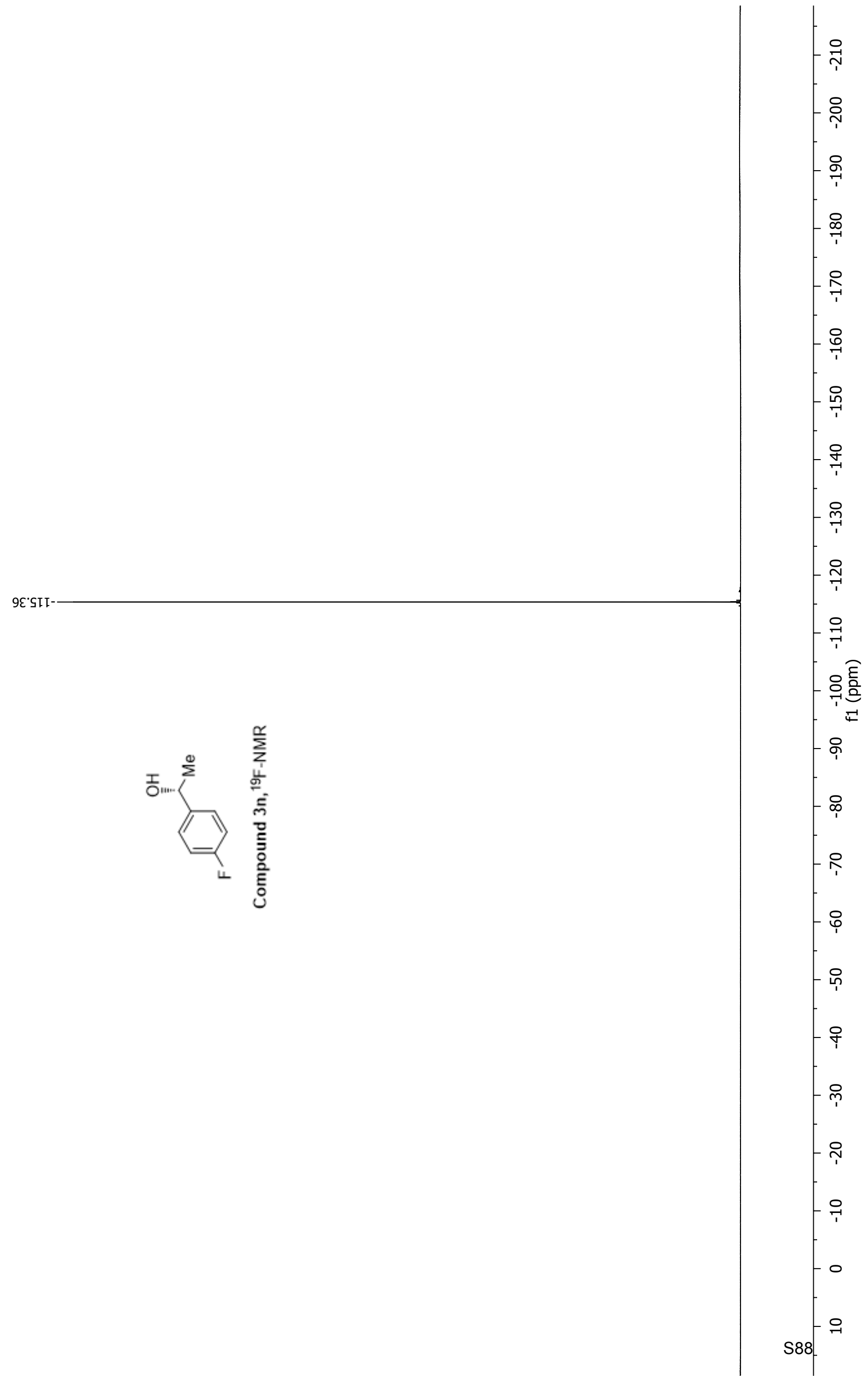


${ }_{6 t \cdot[}^{\angle t}[=$

$78 . \mathrm{T}$

$08^{\circ} \varepsilon-$

$\varepsilon 8^{\circ} t$

$58^{\circ} \mathrm{-}$

$88^{\circ} \textrm{ }$

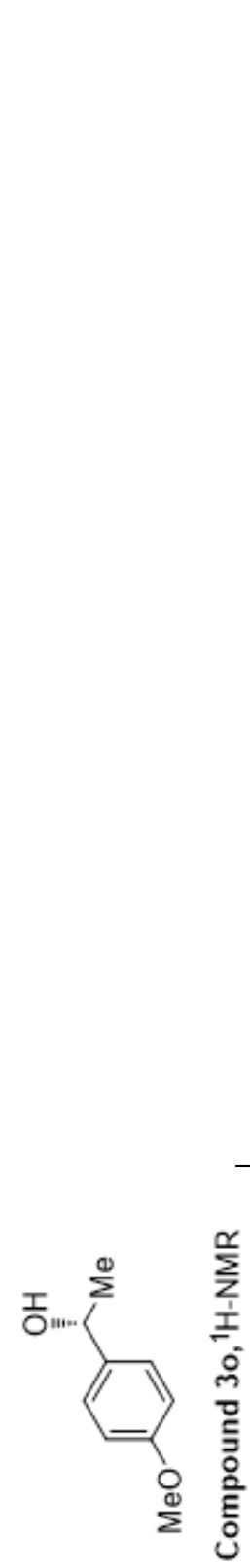

$\angle 8^{\circ} 9$

88.9

68.9 J

$62^{\circ}<$

$0 \varepsilon^{\circ} \angle$

$\left.\begin{array}{l}{[\varepsilon<} \\ {[\varepsilon<}\end{array}\right]$

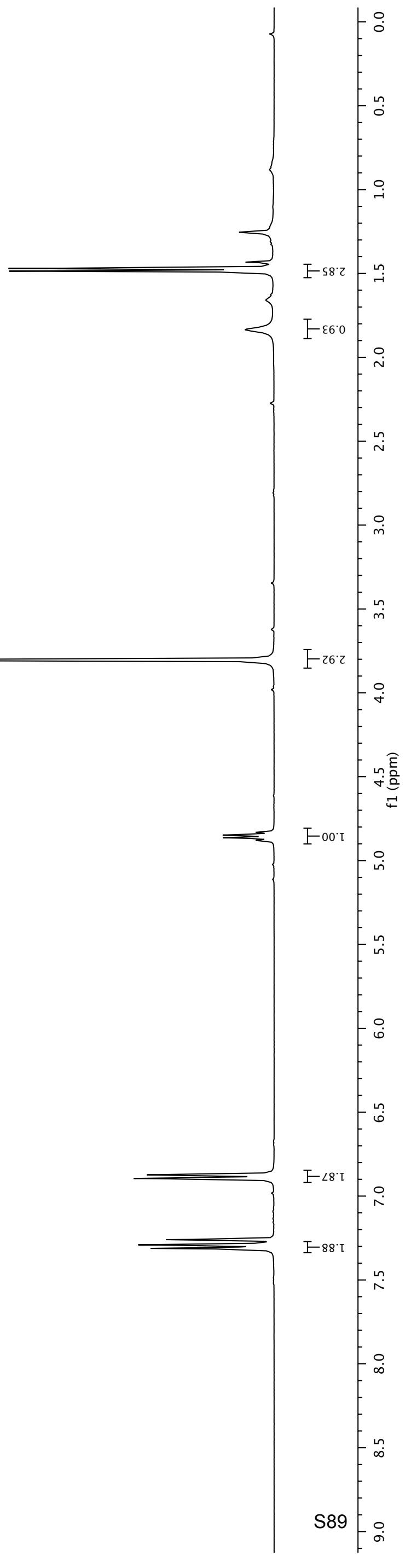


oz'sz-

$9 t^{\prime} \varsigma \varsigma-$

9. $0<-$

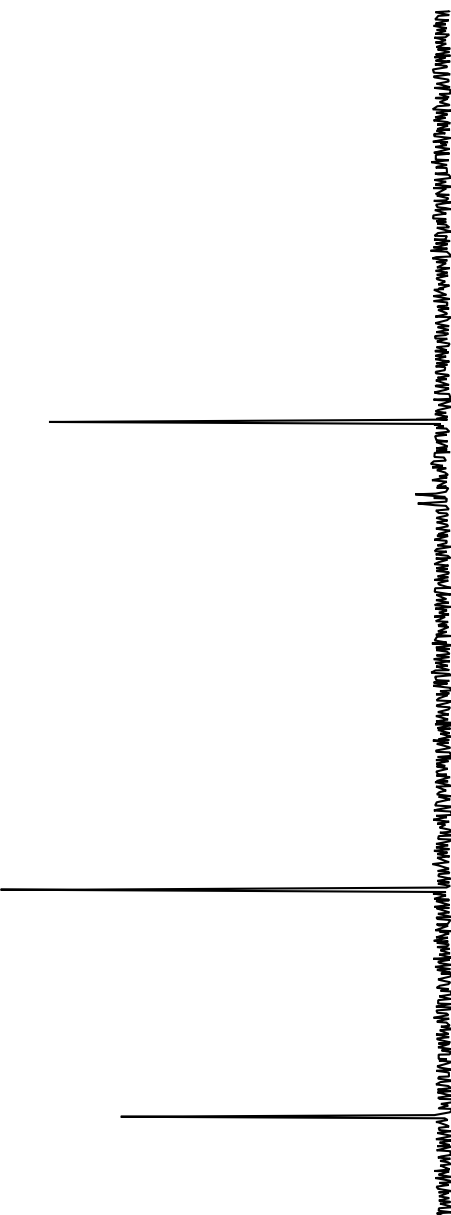

Lo

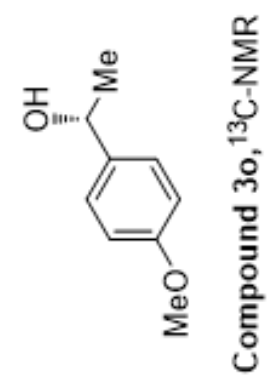

오을

$8^{\circ} \mathcal{E} \mathrm{EI}-$

ع8.92 -

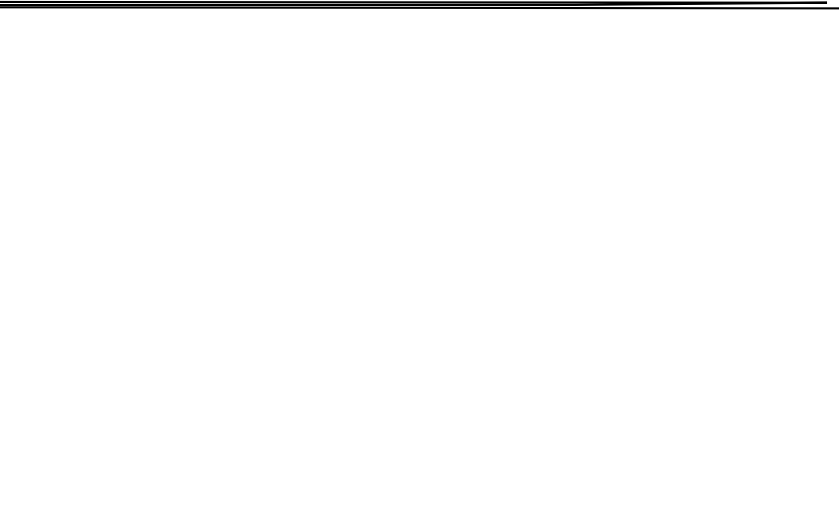

$\varepsilon\left[{ }^{\circ} 8 \varepsilon[-\right.$

I I $6 \mathrm{SI}$

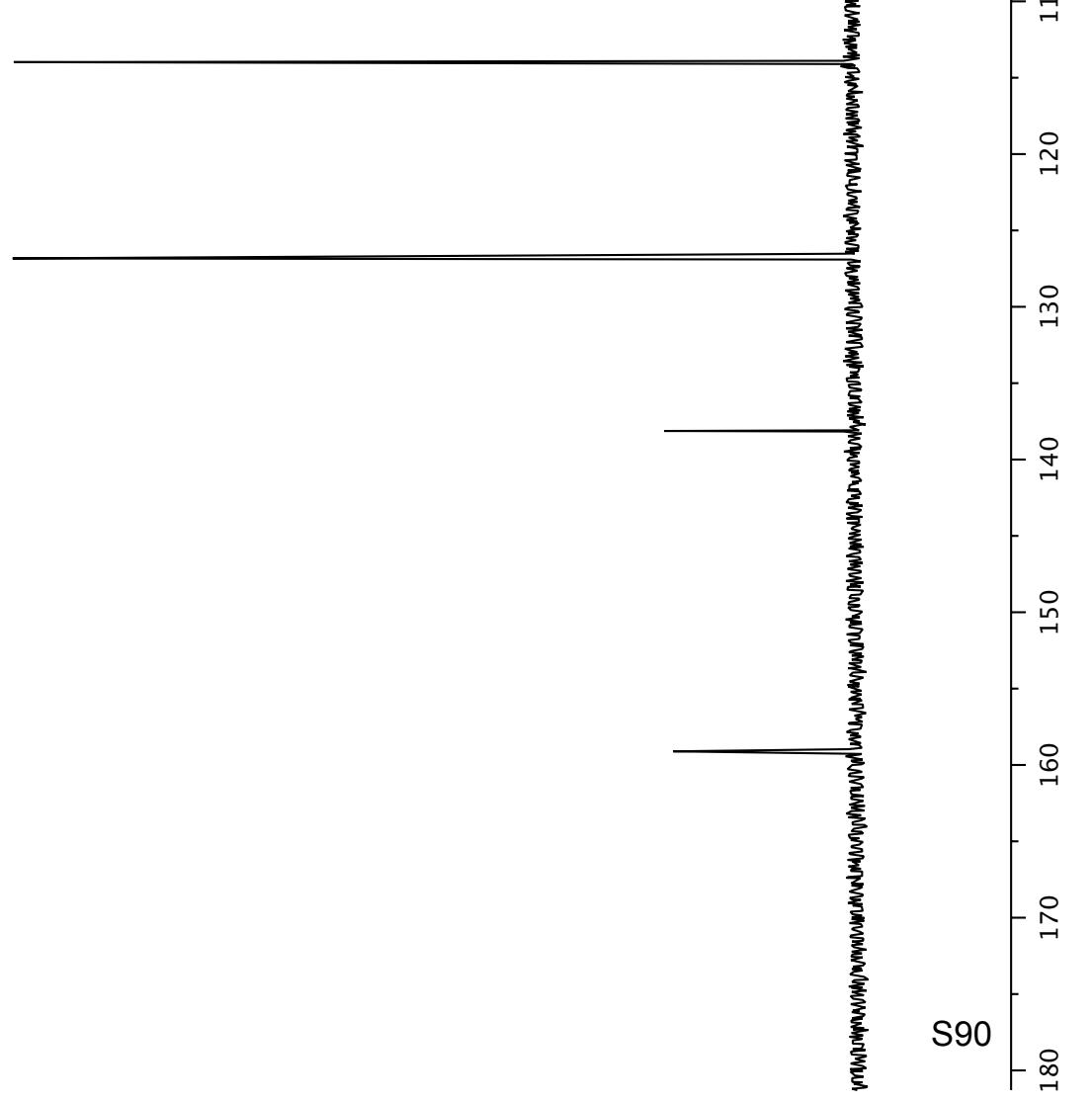


St. $\mathrm{T}$

9L'I-

$08^{\circ}+7$
$28^{\circ} \circ$

s8.

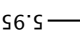

$\left.\begin{array}{l}9 L \cdot 9 \\ 8<\cdot 9 \\ {[8.9} \\ {[8.9} \\ \varepsilon 8.9 \\ \varepsilon 8.9 \\ 06.9 \\ 06.9\end{array}\right]$

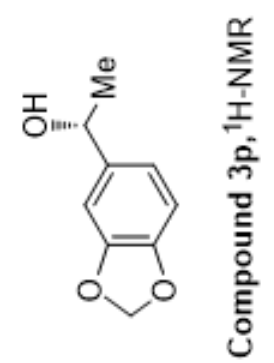

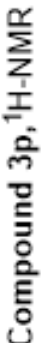

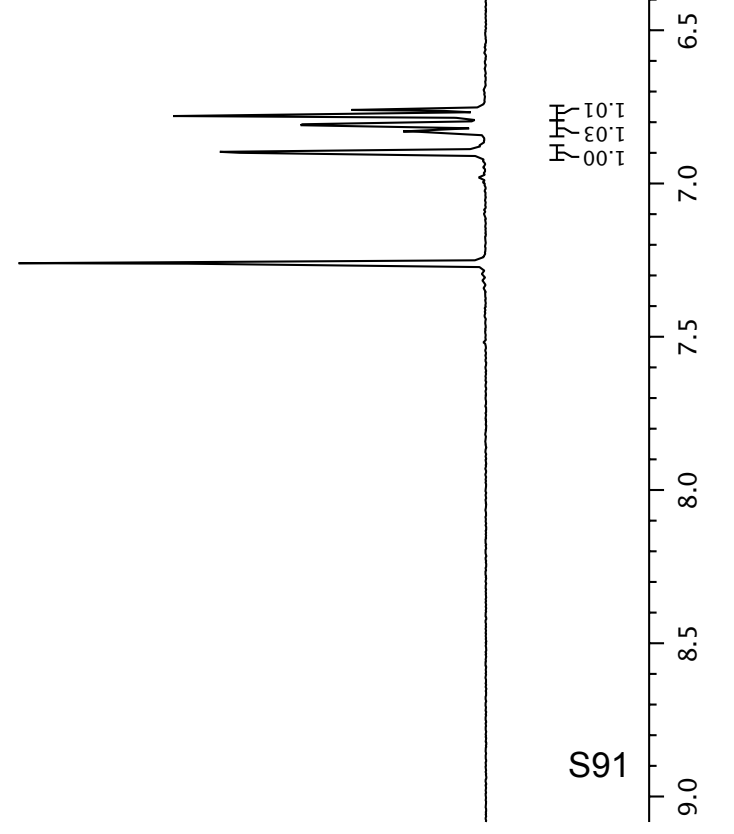




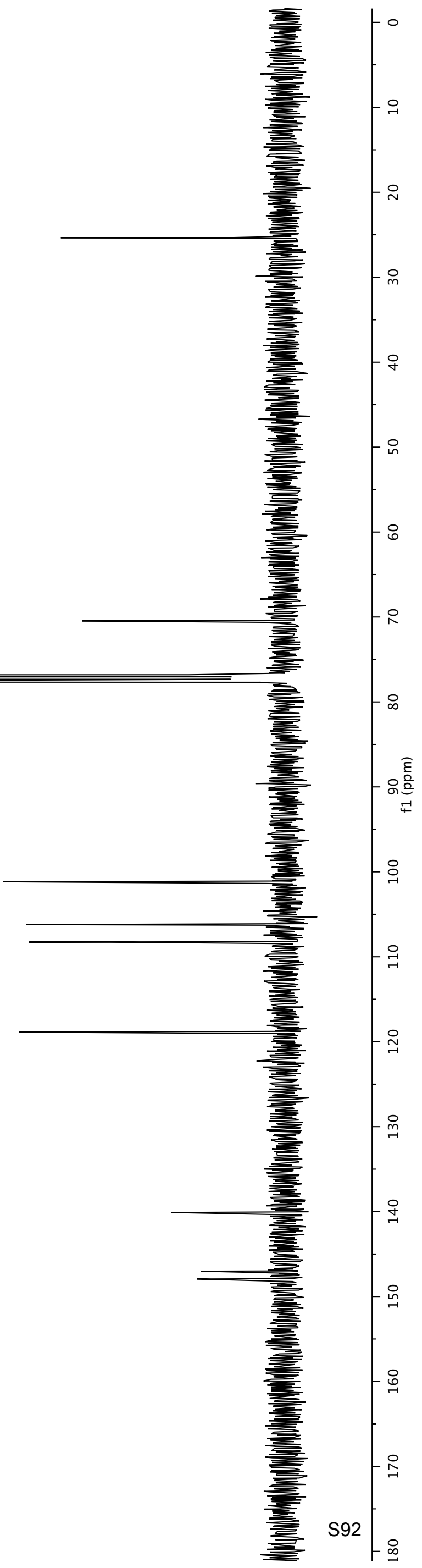



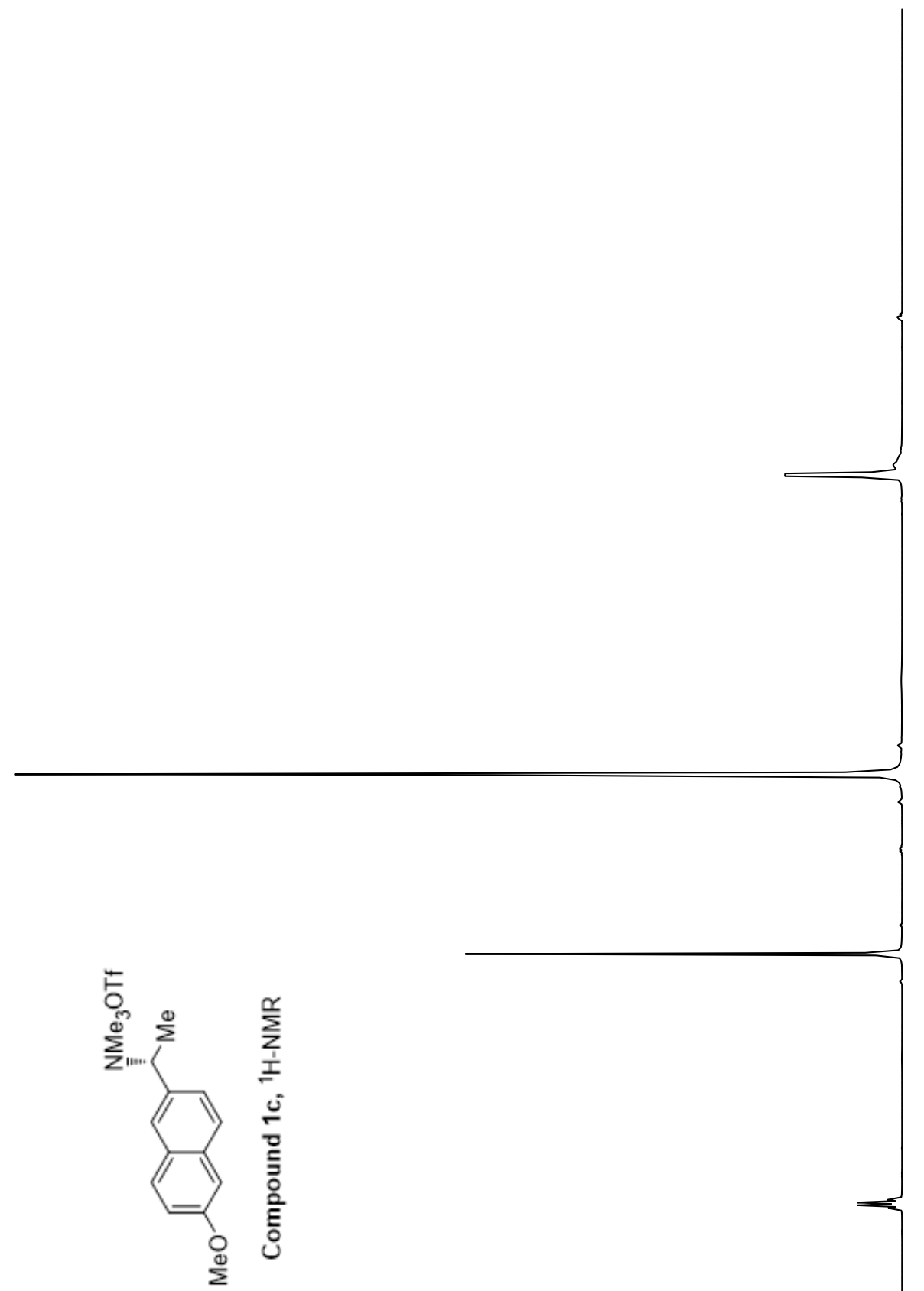

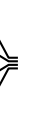

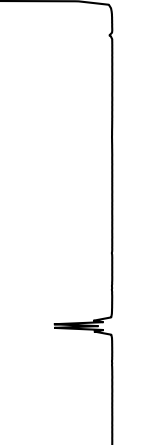


$9 \angle \angle I I-$

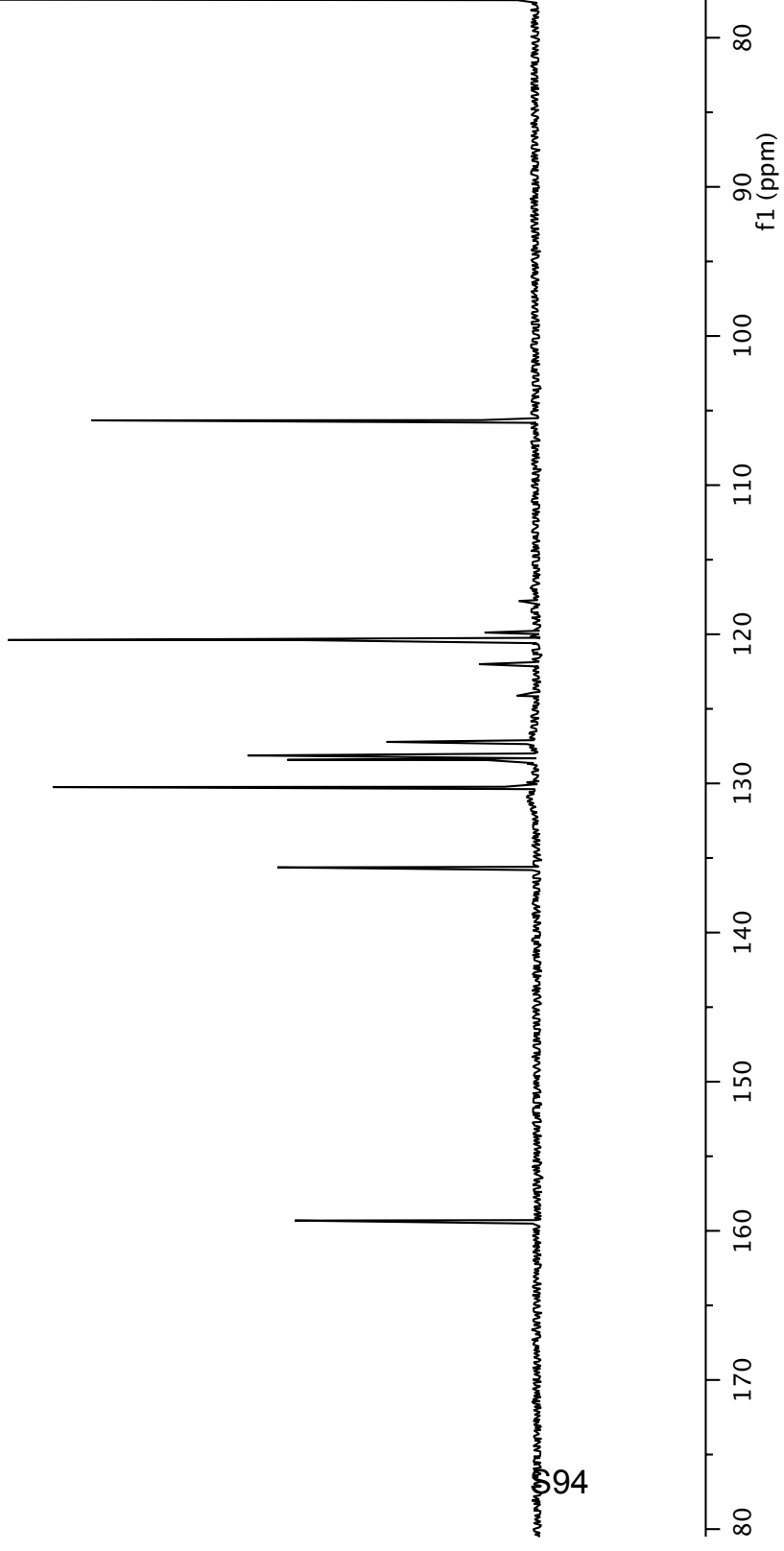




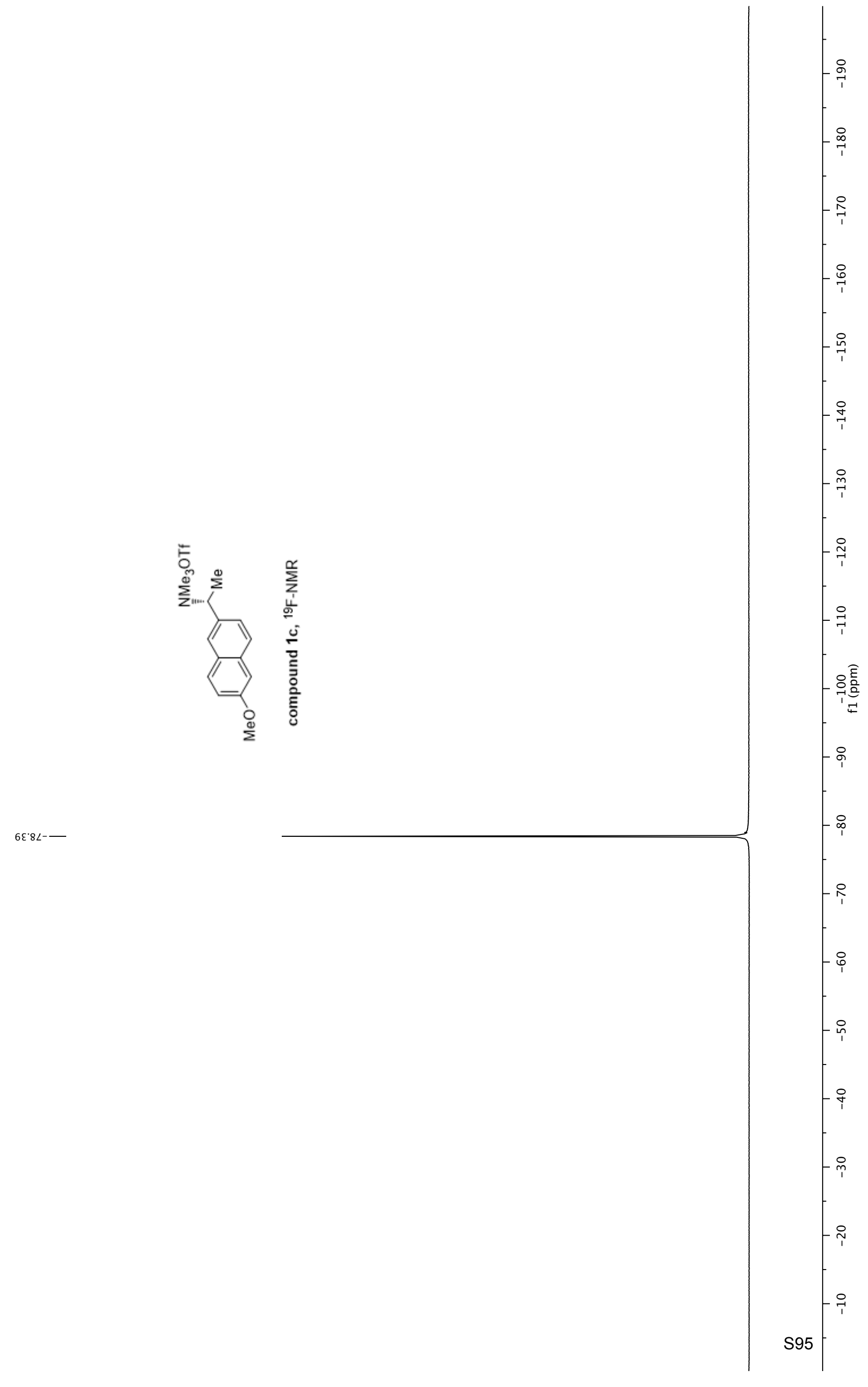




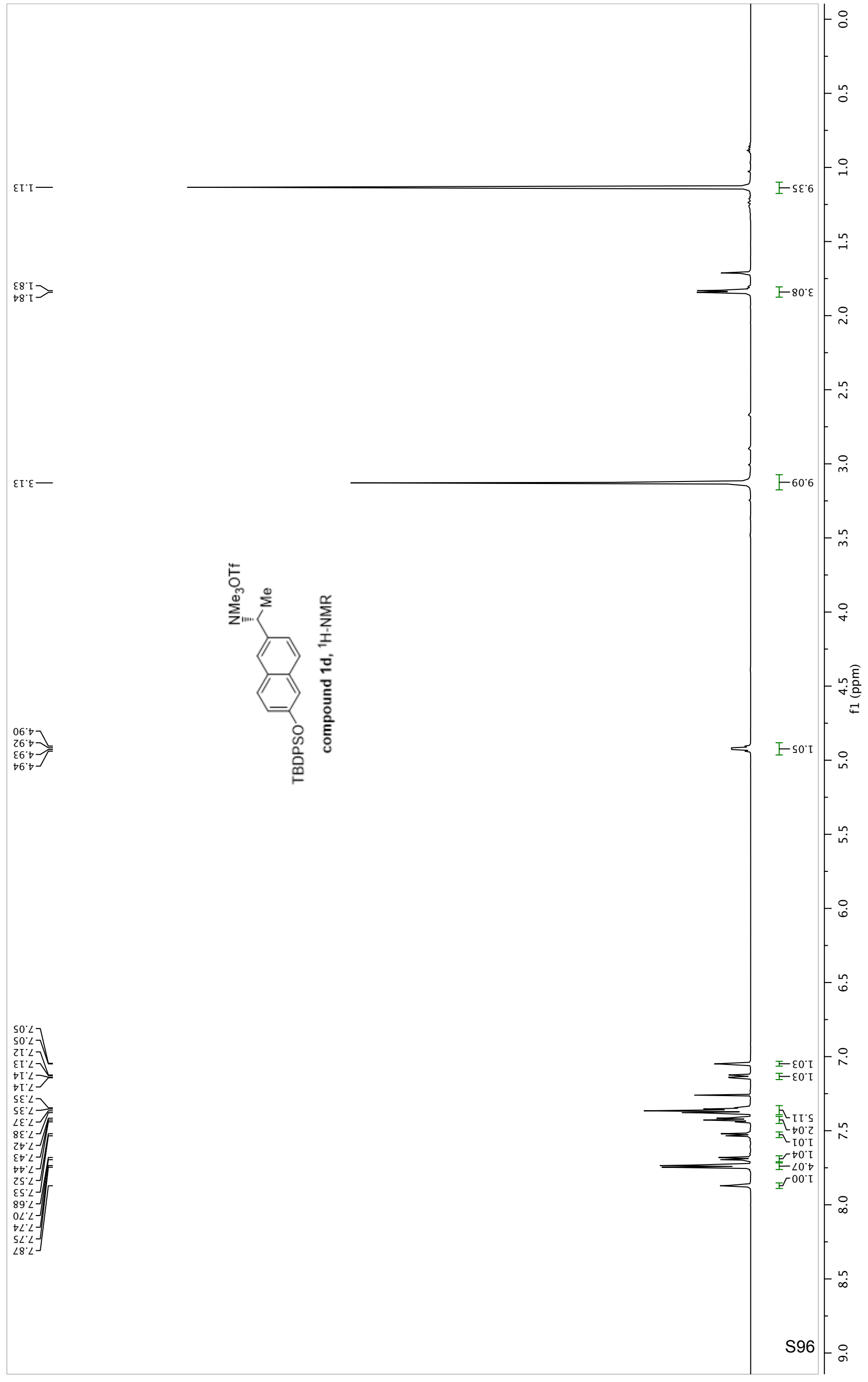


ZZ'SI -

$69^{\circ} 6 \mathrm{I}$

$\angle 9 \cdot 92$

I $Z^{\prime}$ IS

$\varsigma \varsigma^{\circ} \downarrow L$

$85^{\circ} t$ II

I $\angle$ LII ع8.6I I S6.IZI 乙

$62 \cdot \varepsilon 2 \mathrm{CI}$

$\angle 0^{\circ}+2 \mathrm{I}$

$\forall Z^{\circ} \angle Z[$

$\rightarrow \mathrm{L} 8 \mathrm{ZI}$

87.821

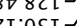

62.0ह[

6ร'حहा

$9 \varepsilon$ ' ऽ

†9' ऽEI

$6 Z^{\circ}$ SSI -

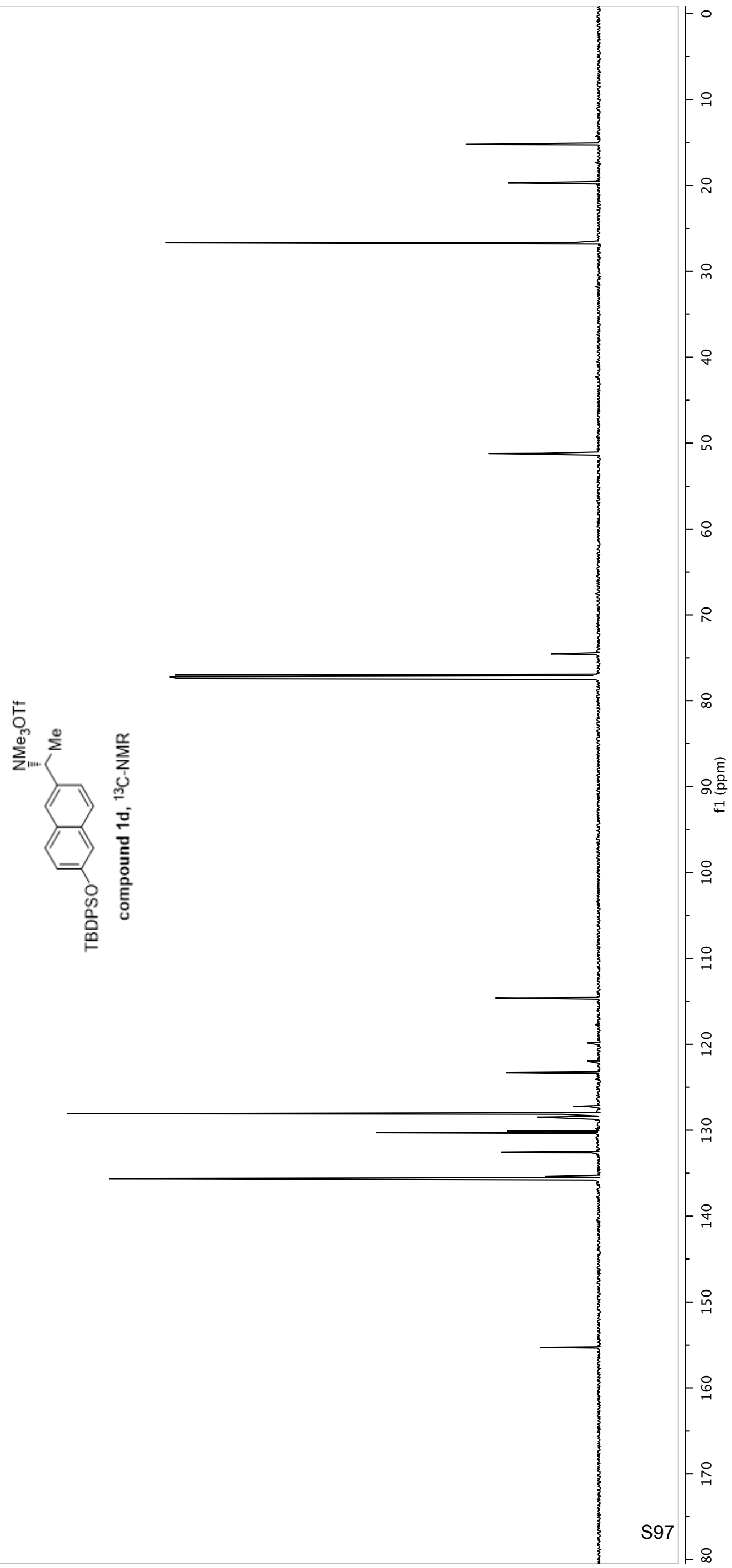




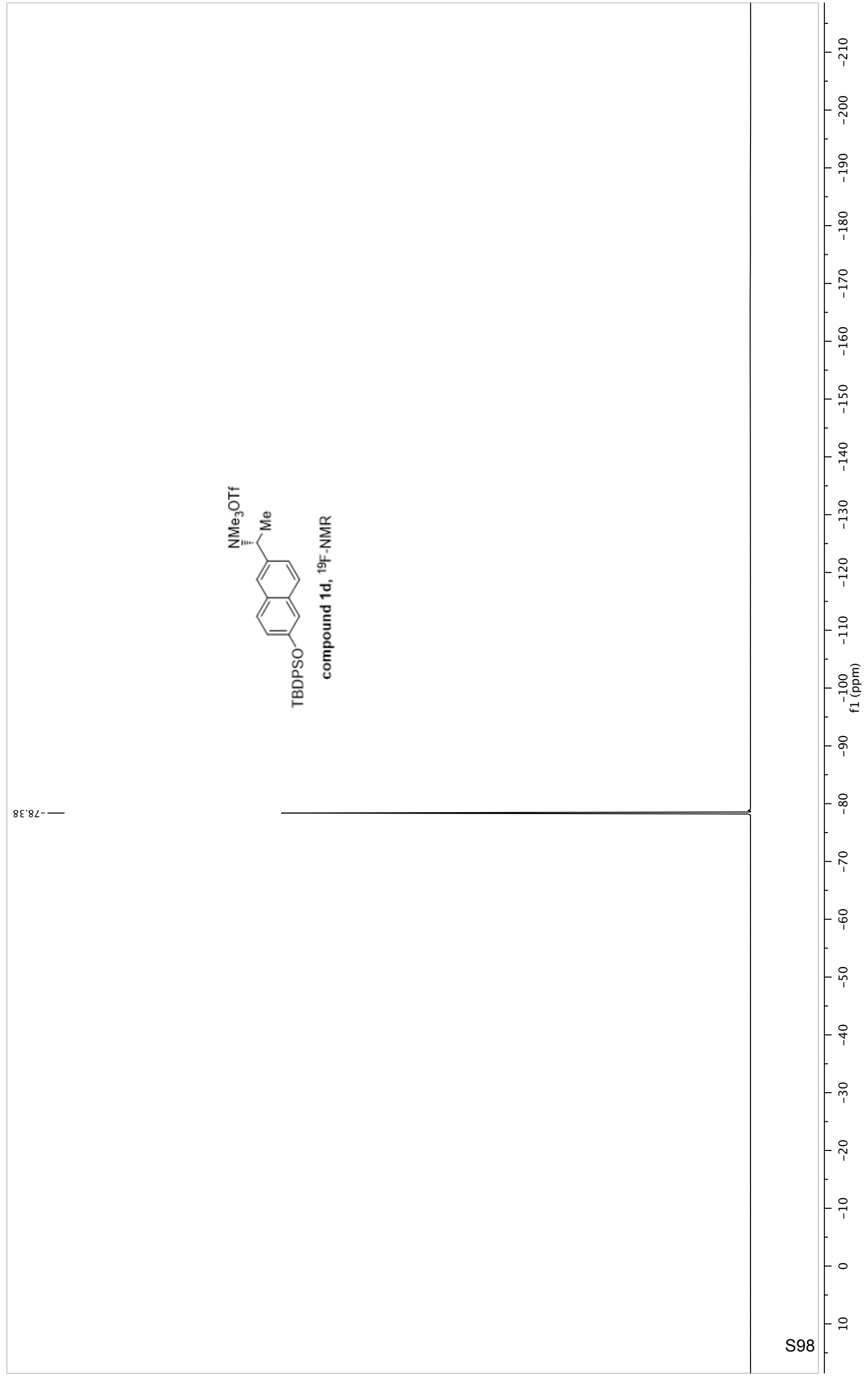




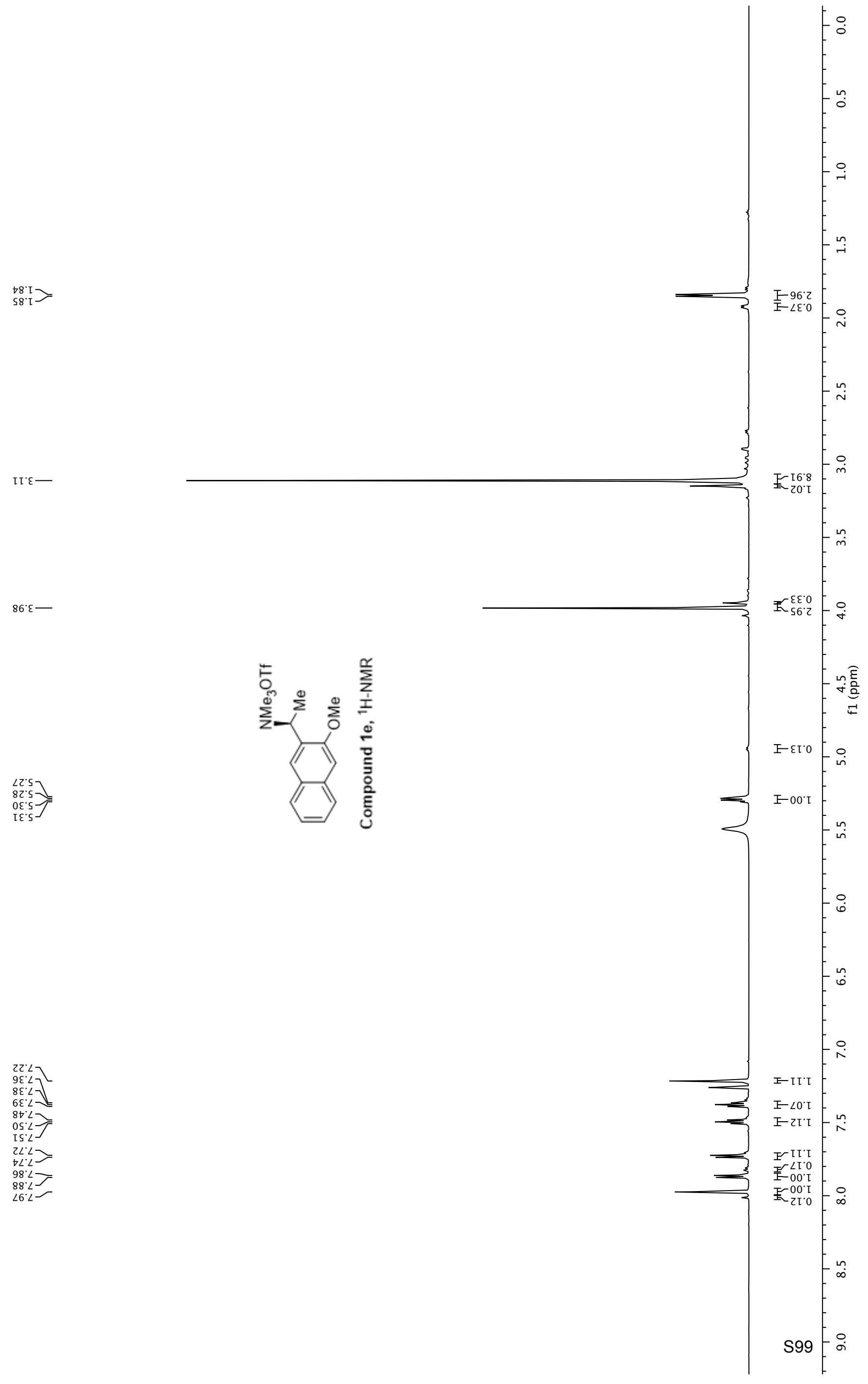




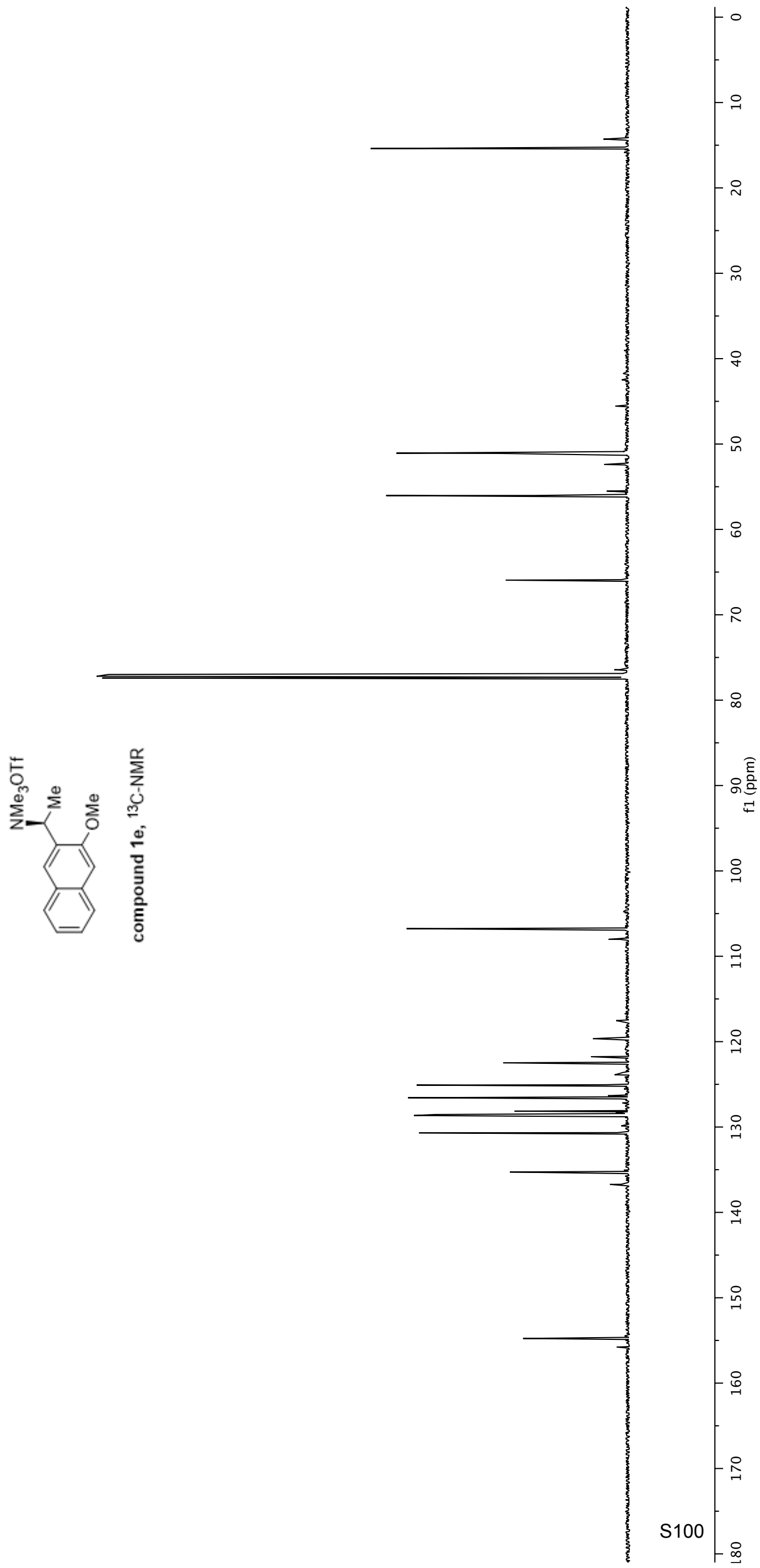




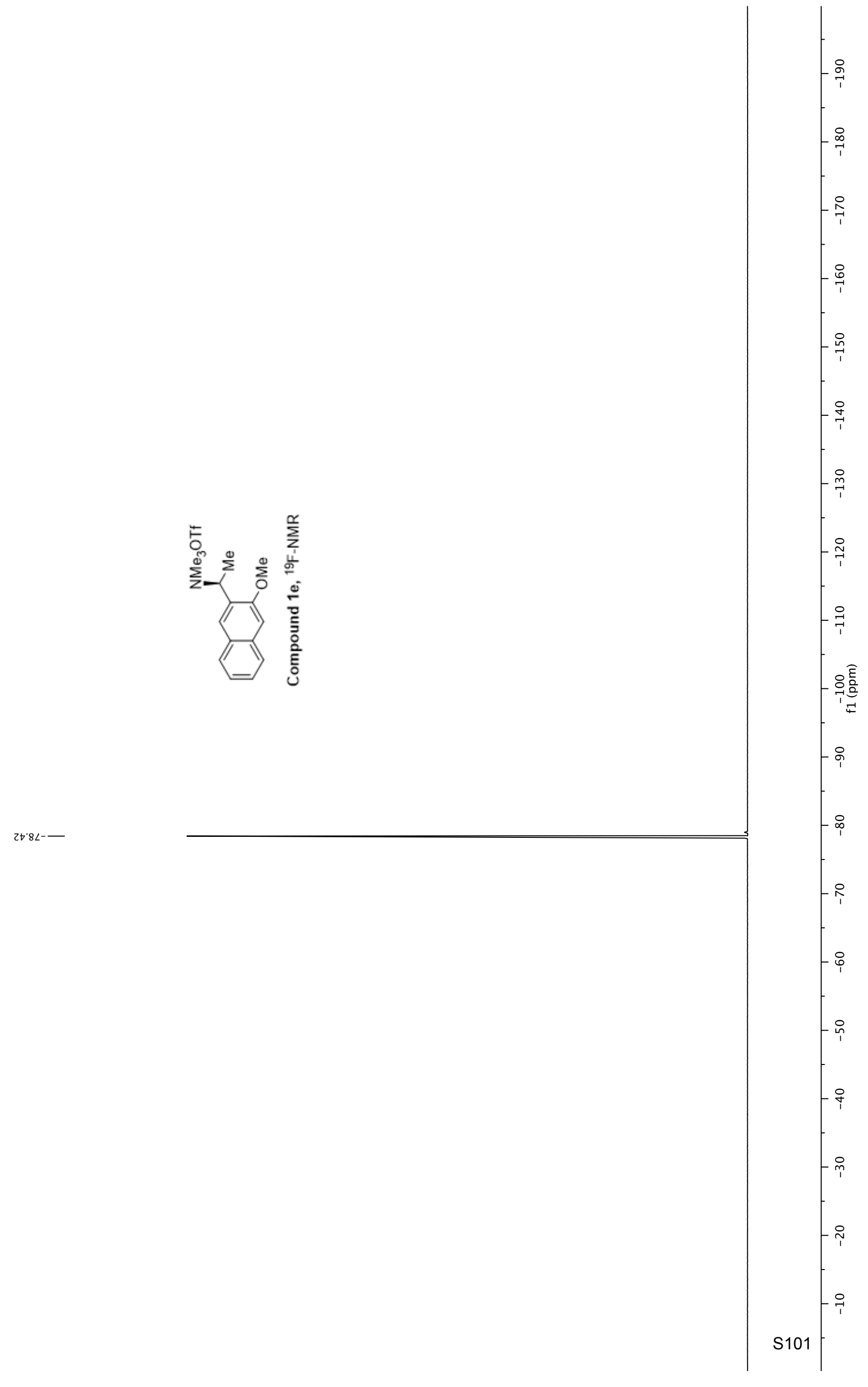




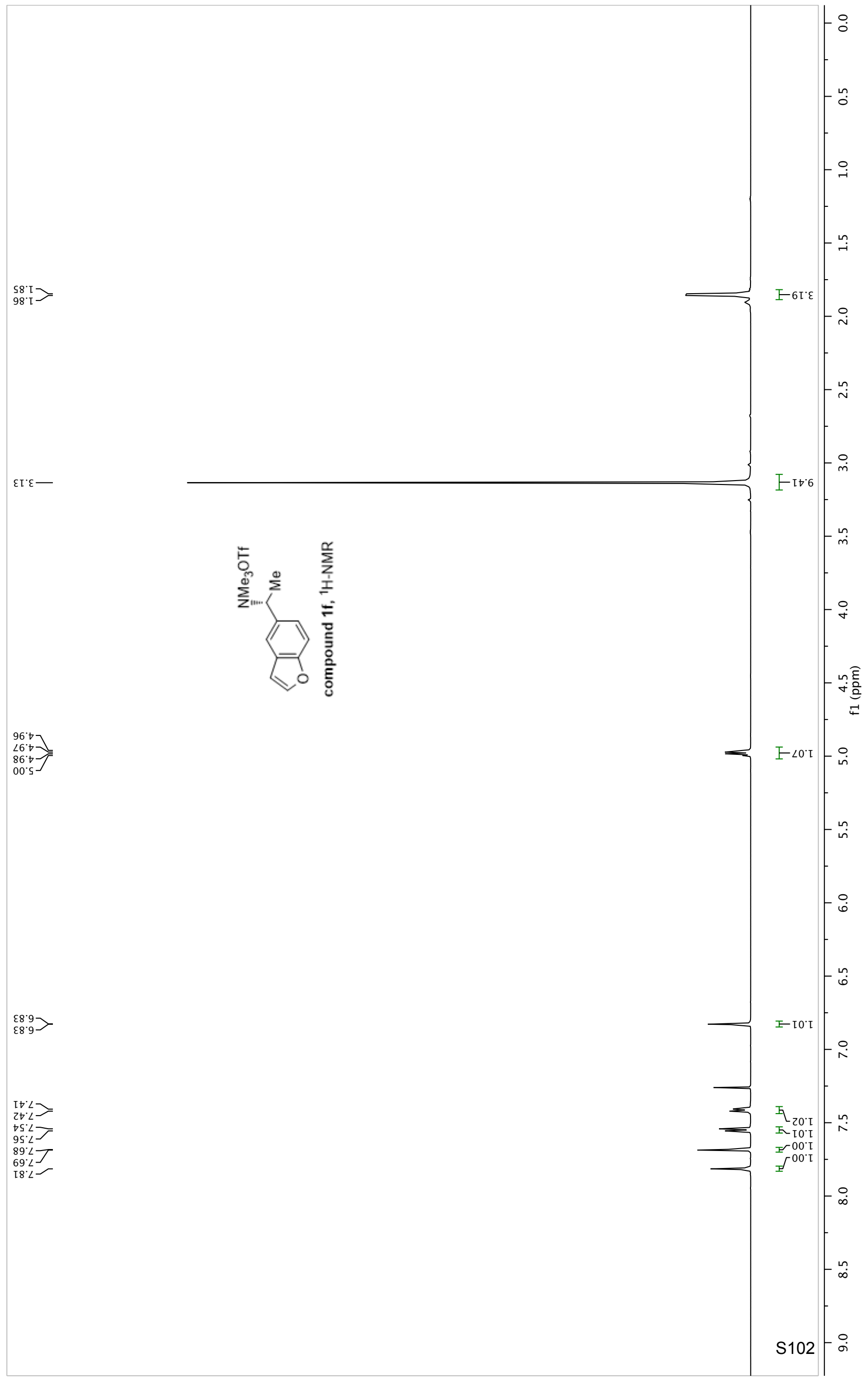




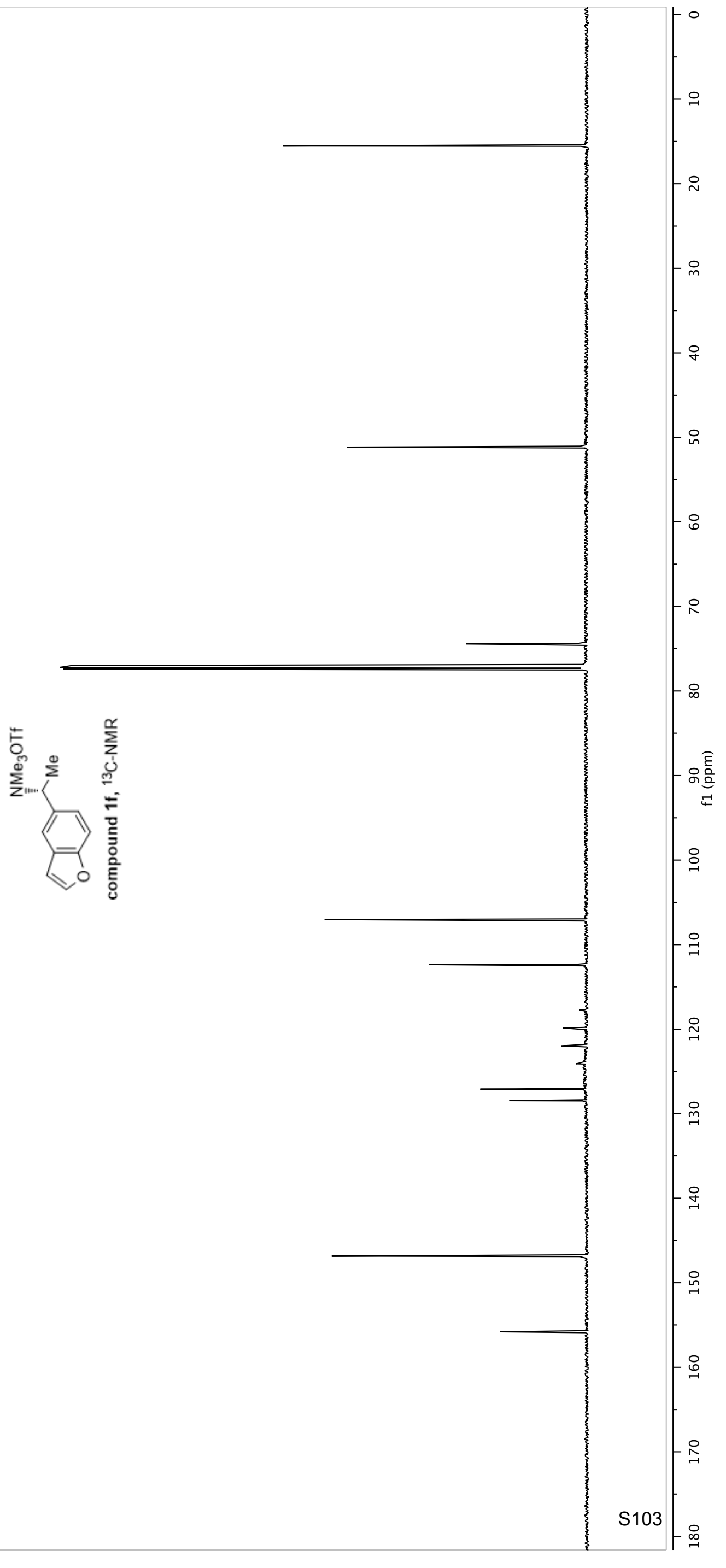

t0 $\angle 0 \mathrm{I}$

9ع'zI L

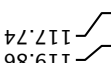

98.6II

86. IZI -

$60 . \angle 2 \mathrm{I}$

$9 t^{\circ} 8217$

$98 \cdot 9 t \mathrm{I}$

I8.SSI - 


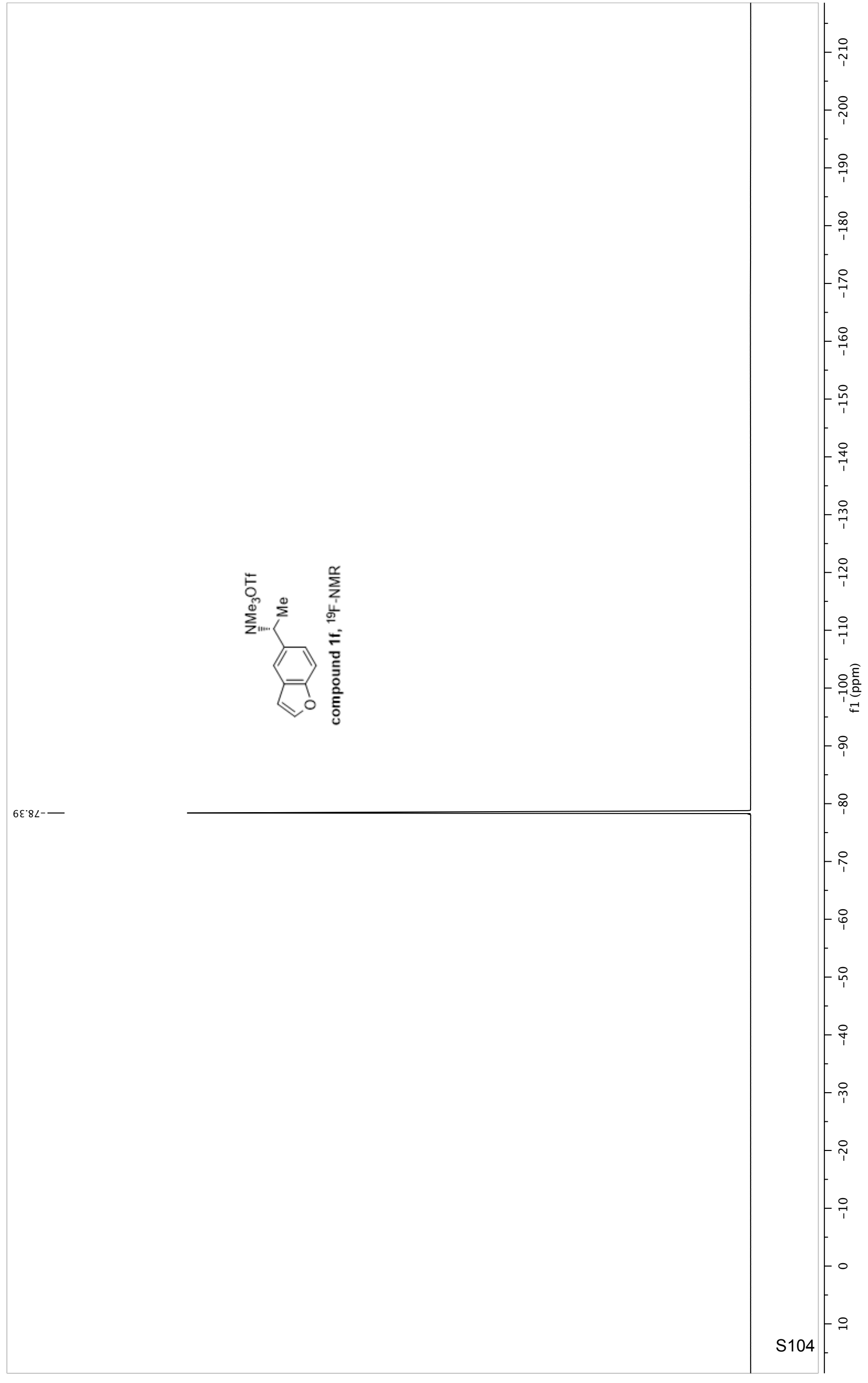




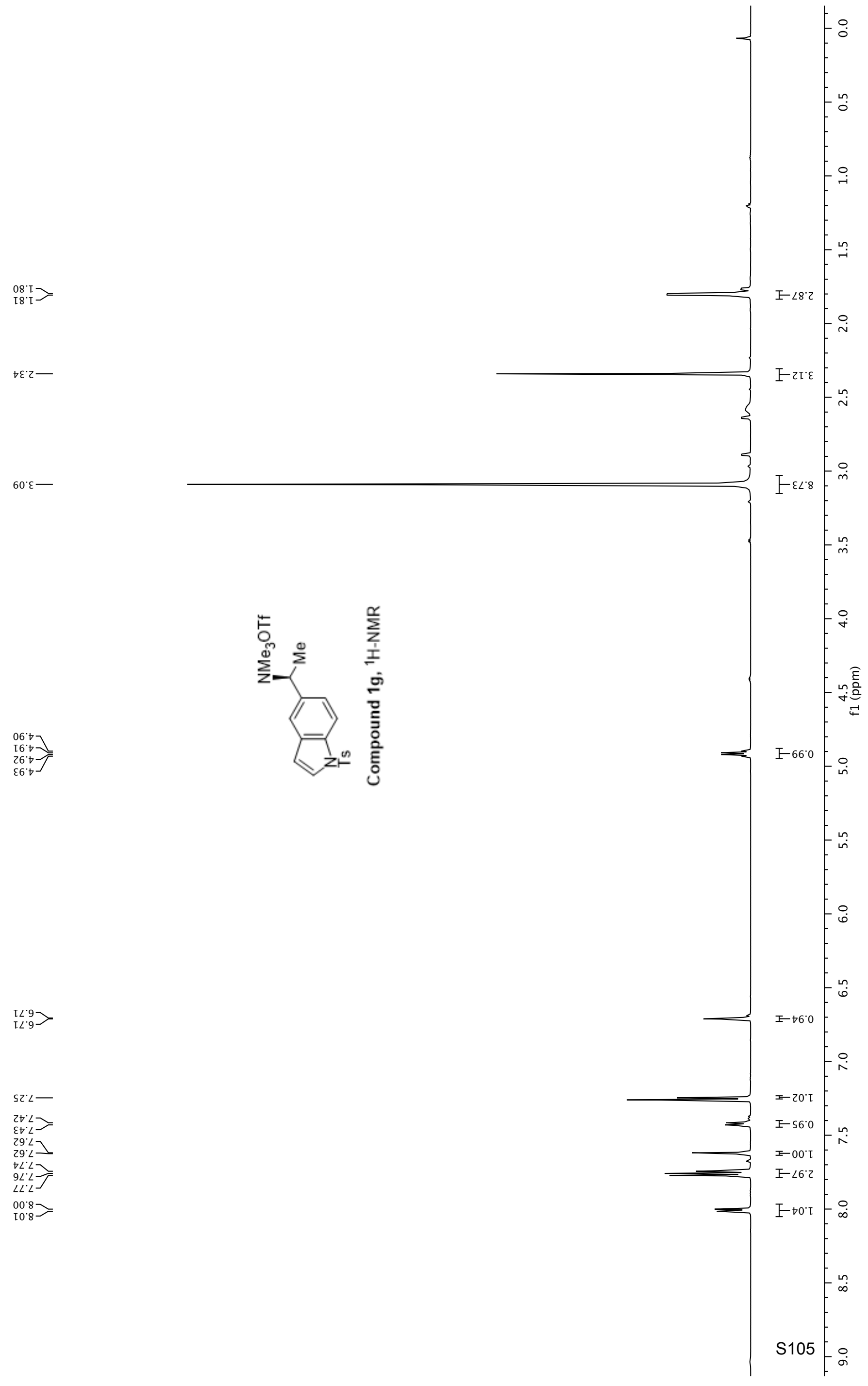


$6 \varepsilon^{`} \mathrm{SI}$

$8 L$ L2 -

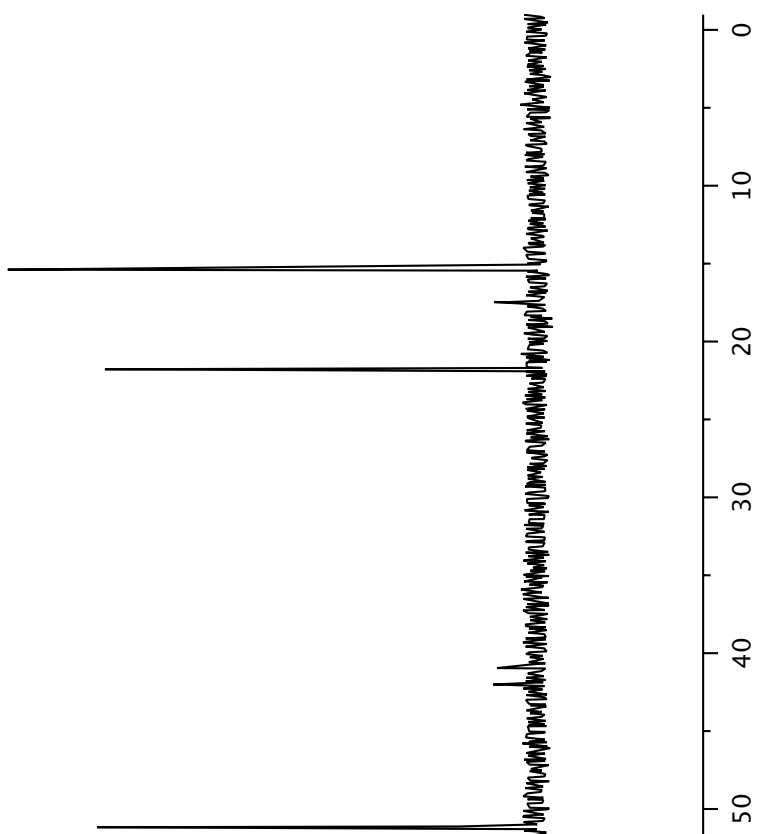

LI'IS-

$โ \varepsilon^{\circ} \sqcup<-$

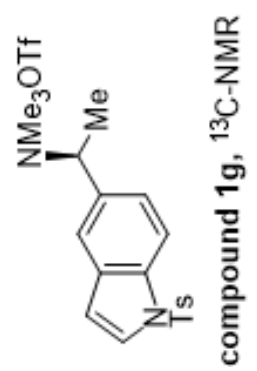

हิ

50.60I-

9ז'ㄷㄷ

$\angle 9 \circ \mathrm{LI}-$

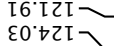

$\varepsilon 0^{\circ} \circ Z[$
$90^{\circ} \angle Z I$

$\left.\begin{array}{l}90^{\circ} \angle Z[ \\ 6 \varepsilon^{\circ} \angle Z[]\end{array}\right]$

$86^{\circ} \angle Z I$

$9 \varepsilon^{\circ} 0 \varepsilon \mathrm{L} \backslash$
$\angle \mathrm{C}^{\circ} \mathrm{IEL}$

$86^{\circ} \downarrow \varepsilon I-$

६9. ऽ६I -

$6 L \operatorname{st} I$

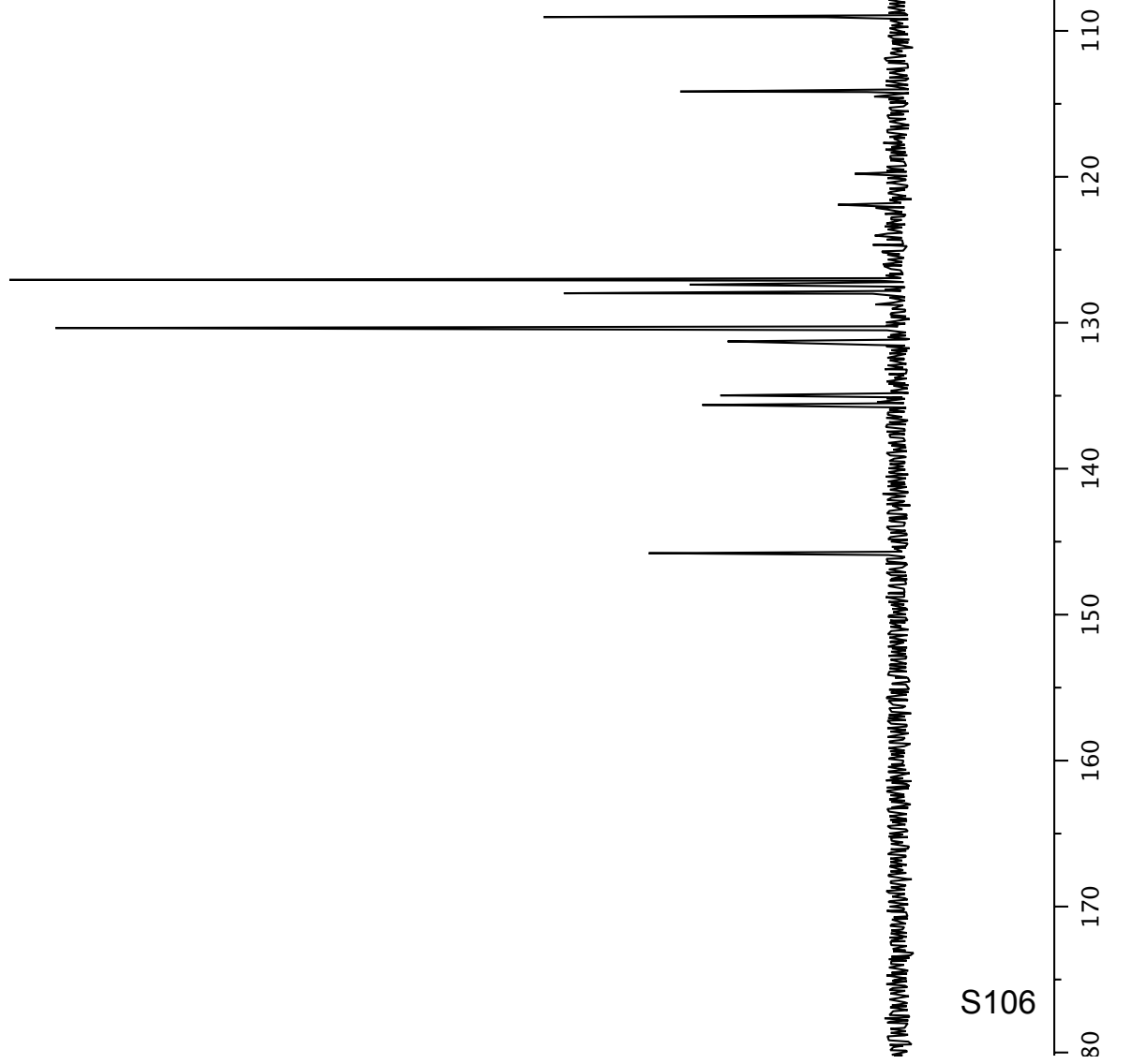




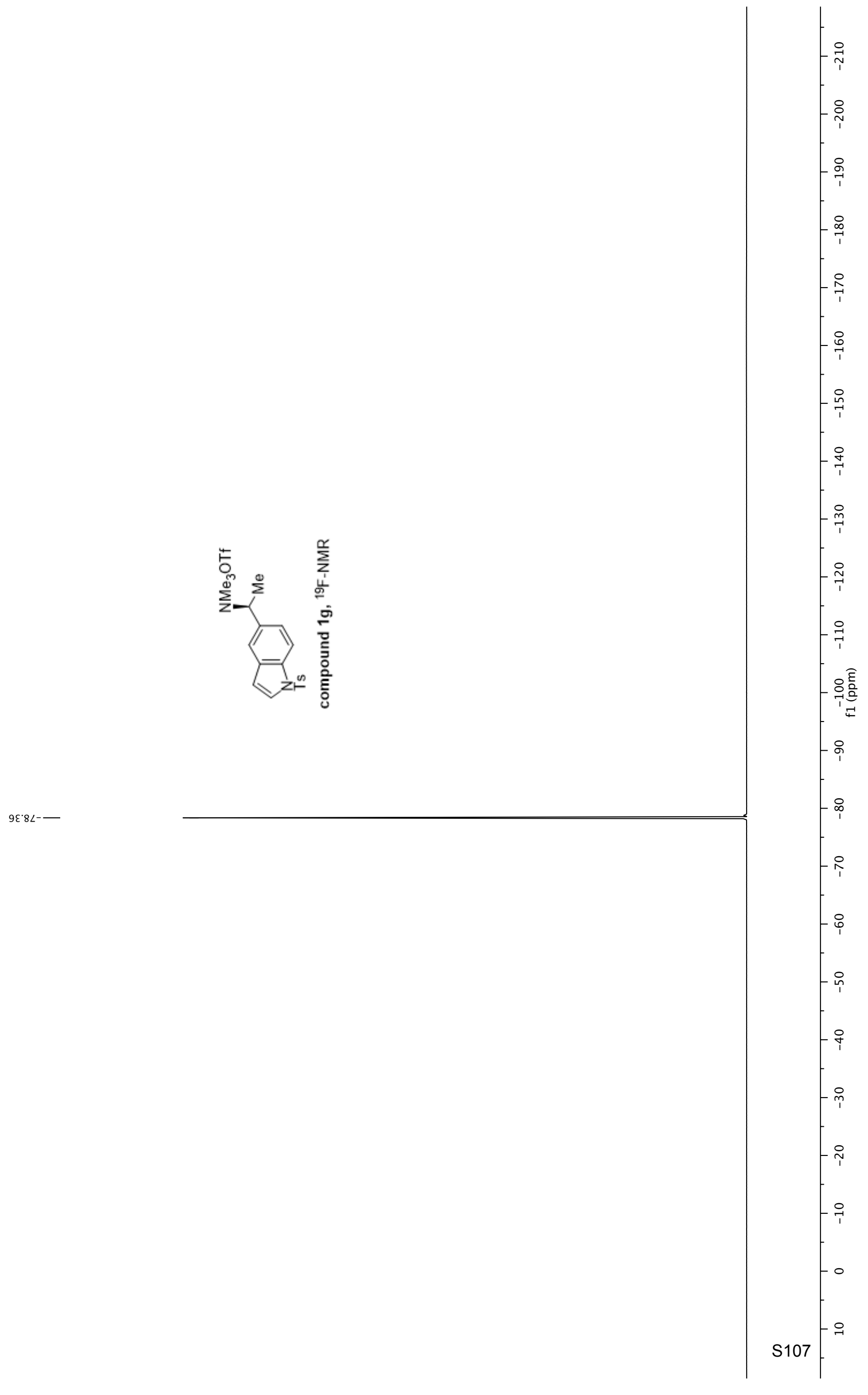



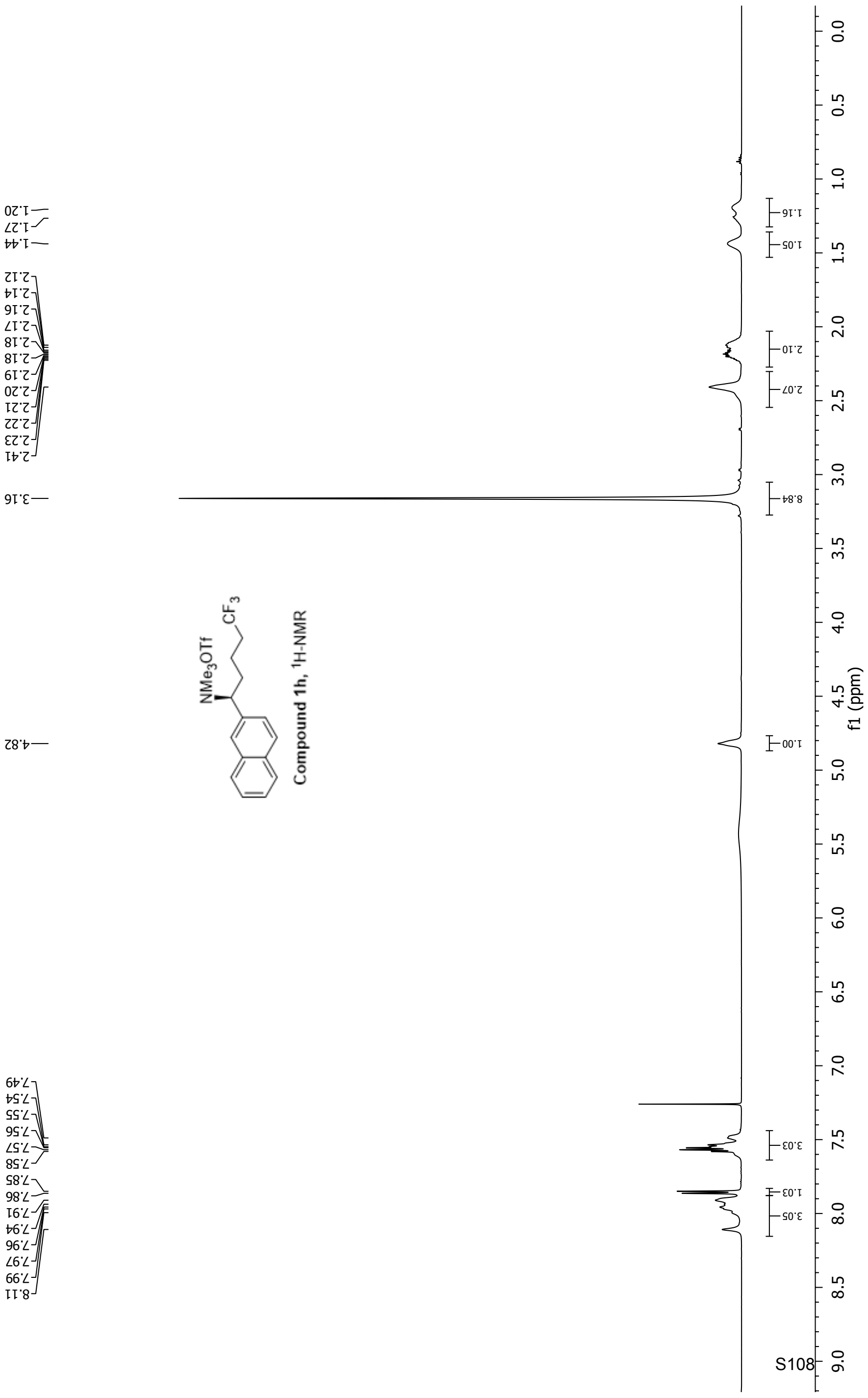


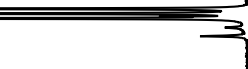

$86^{\circ} \mathrm{SII}$

SI' $6 \mathrm{II}$

¿ะ'حZI

$\angle L ' Z Z I$

$\angle 8$ ZZI]

OS'SZI]

ZS'SZT]

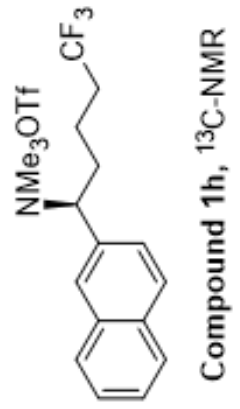

¿S'LZT]

98'LZT]

¿Z'8ZT

$\angle Z{ }^{\circ} 8 \mathrm{ZI}$

$\varepsilon \angle ' 8 Z \mathrm{I}$

ع9'6ZI-

†०००ᄃ $-F$

โO ${ }^{\circ}$ IEI

OI'E६โ

$\downarrow I^{\circ} \forall \varepsilon I-$

$86^{\circ} \sqcup \varepsilon I-$

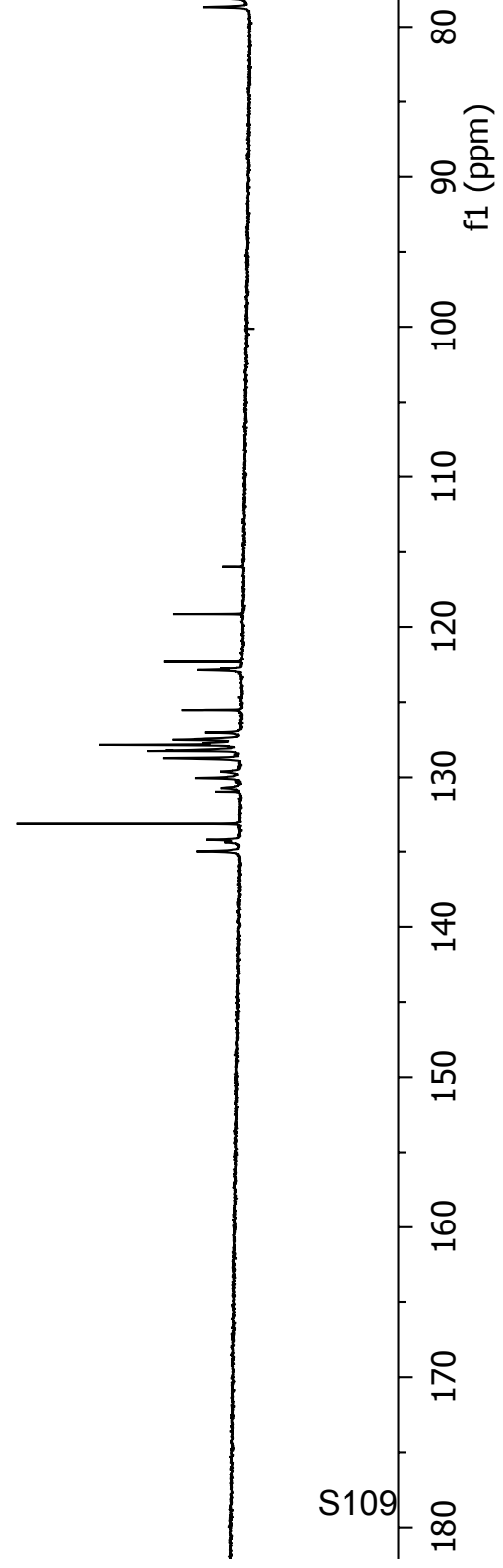


(mdd) If

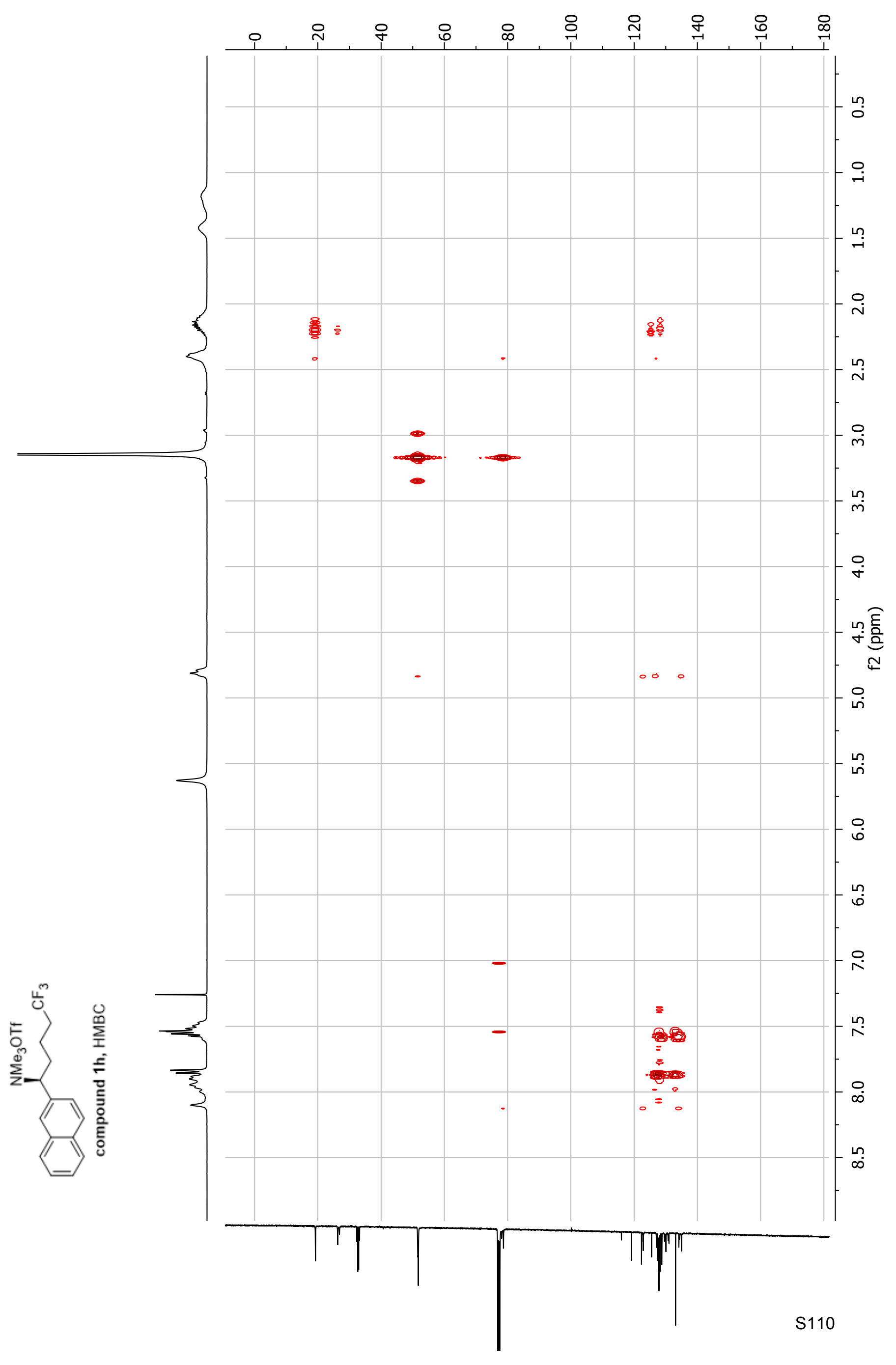


(mdd) If

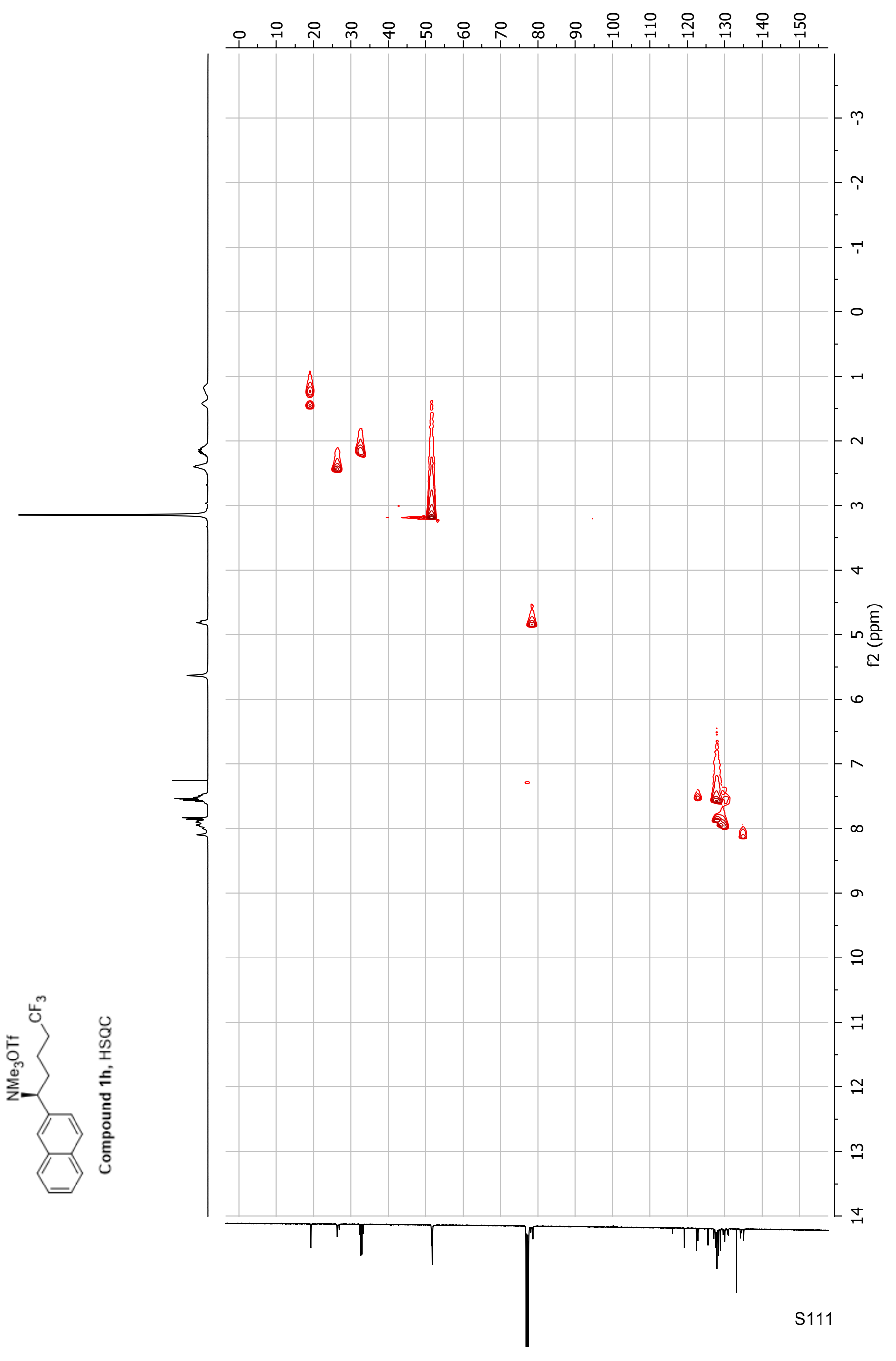




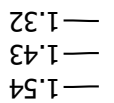

$\angle \varepsilon^{\prime} Z-$

$t t^{2} 2$

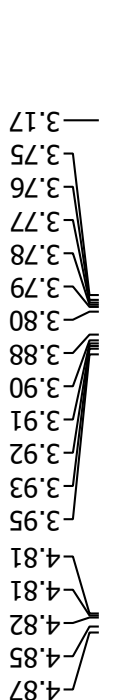

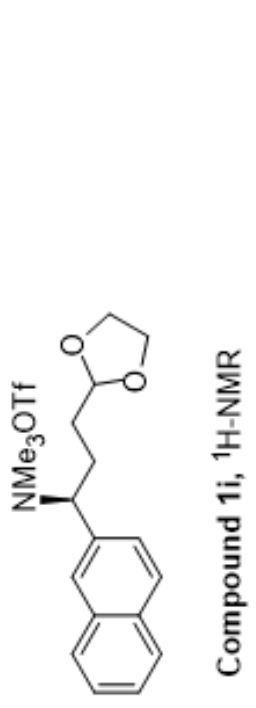

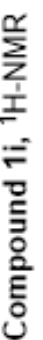
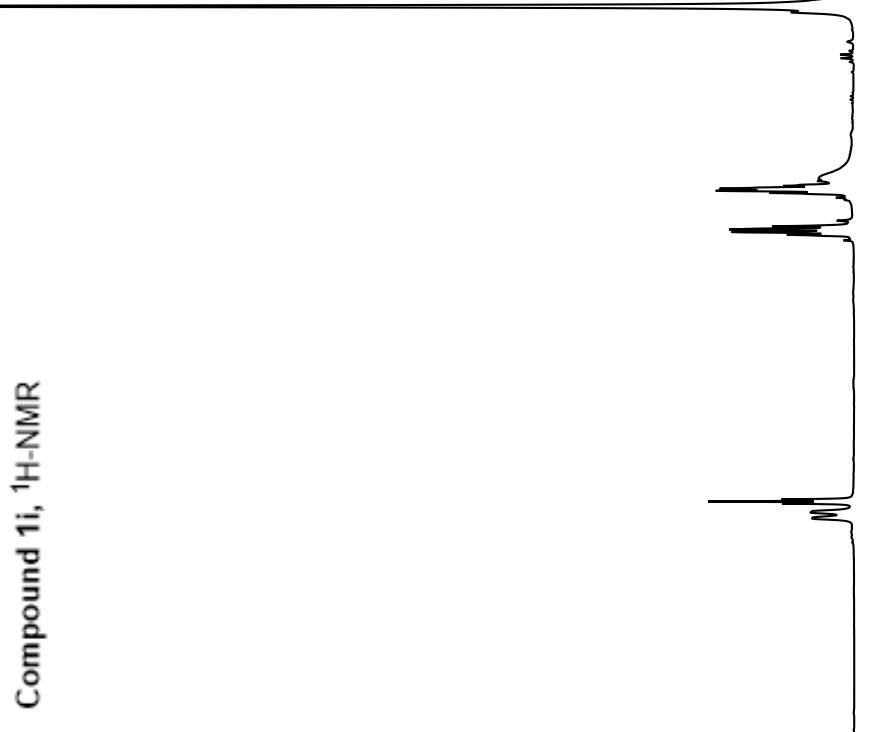

T-s 8

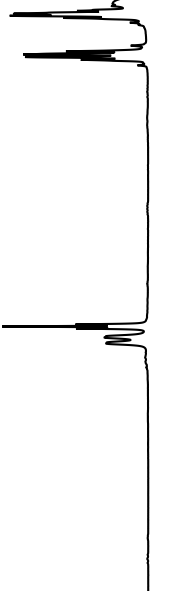

Ft8.

$-0$

$-\stackrel{0}{0}$

96 '.

$\stackrel{n}{\text { N }}$

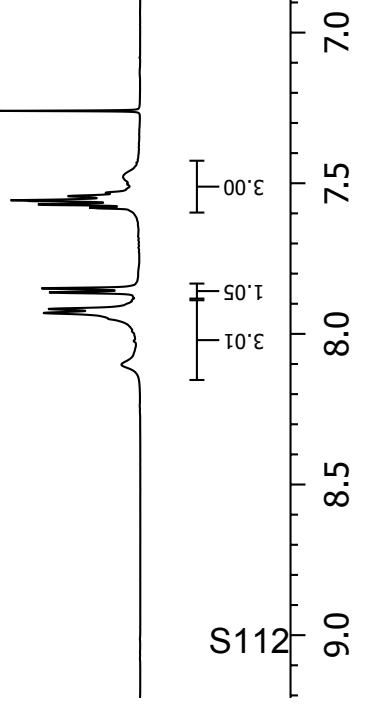




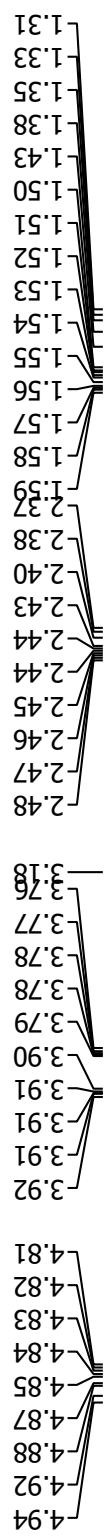

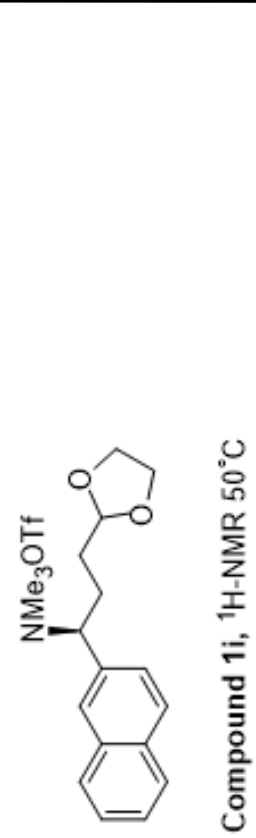

ZS:

$\varepsilon \varsigma^{\circ} L$

$\forall S^{\circ} L$

$\forall S: L$

SS'L

$9 S^{\circ} \angle$

$\angle S^{\circ} \angle$

$\angle S^{\circ} \angle$

65'L

$58{ }^{\circ} \mathrm{L}$

$98^{\circ} \mathrm{L}$

$\angle 8^{\circ} \mathrm{L}-$

$\left.\varepsilon 6^{\circ} \mathrm{L}\right]$

$56{ }^{\circ} \mathrm{L}$

90.8
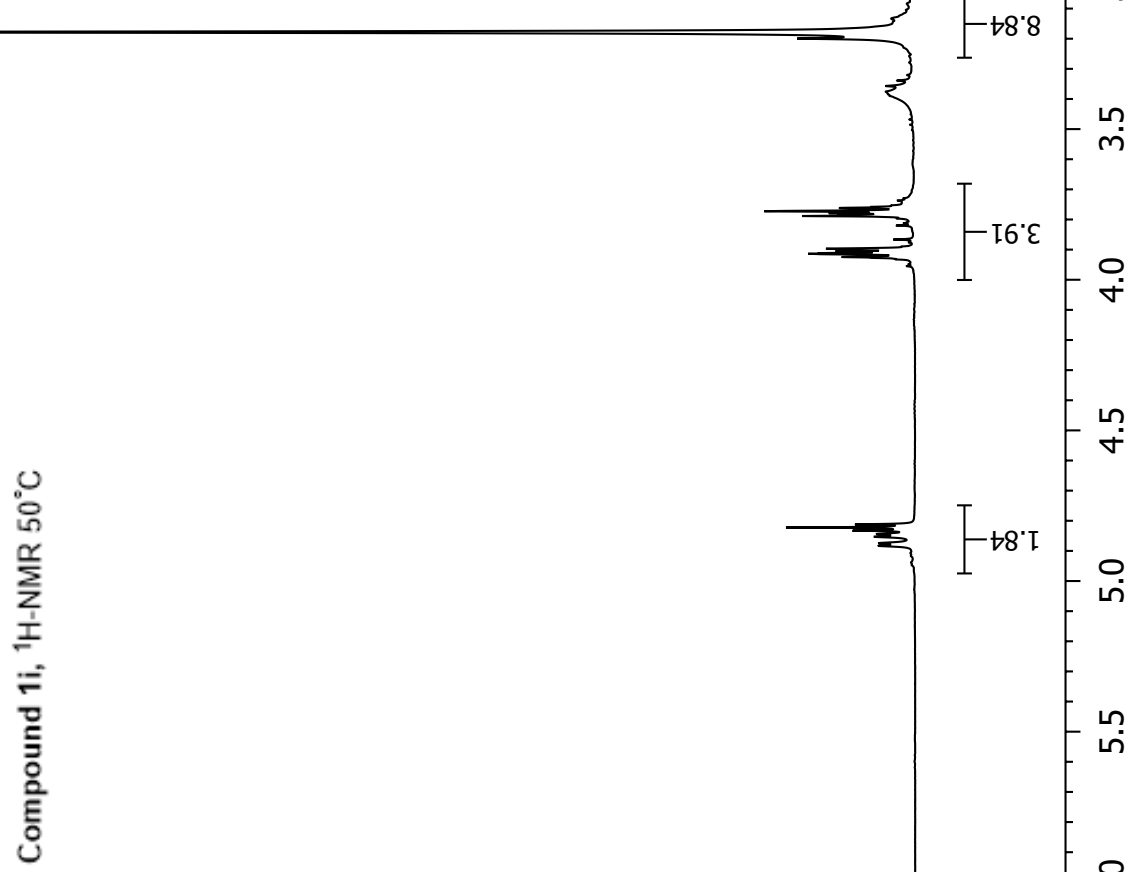

นกิ

우

เก

-

ᄂ

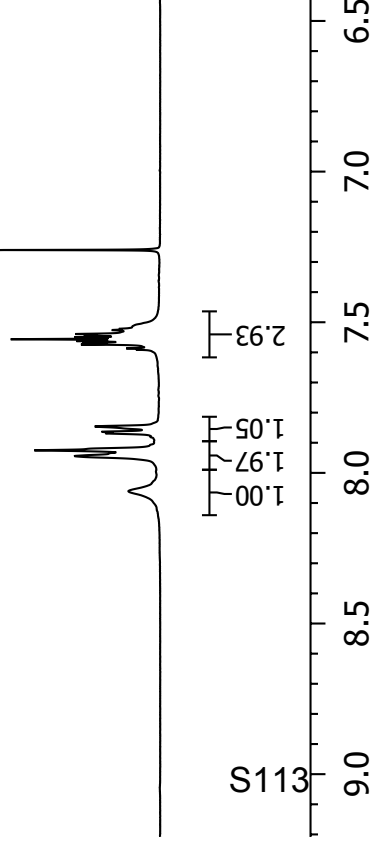




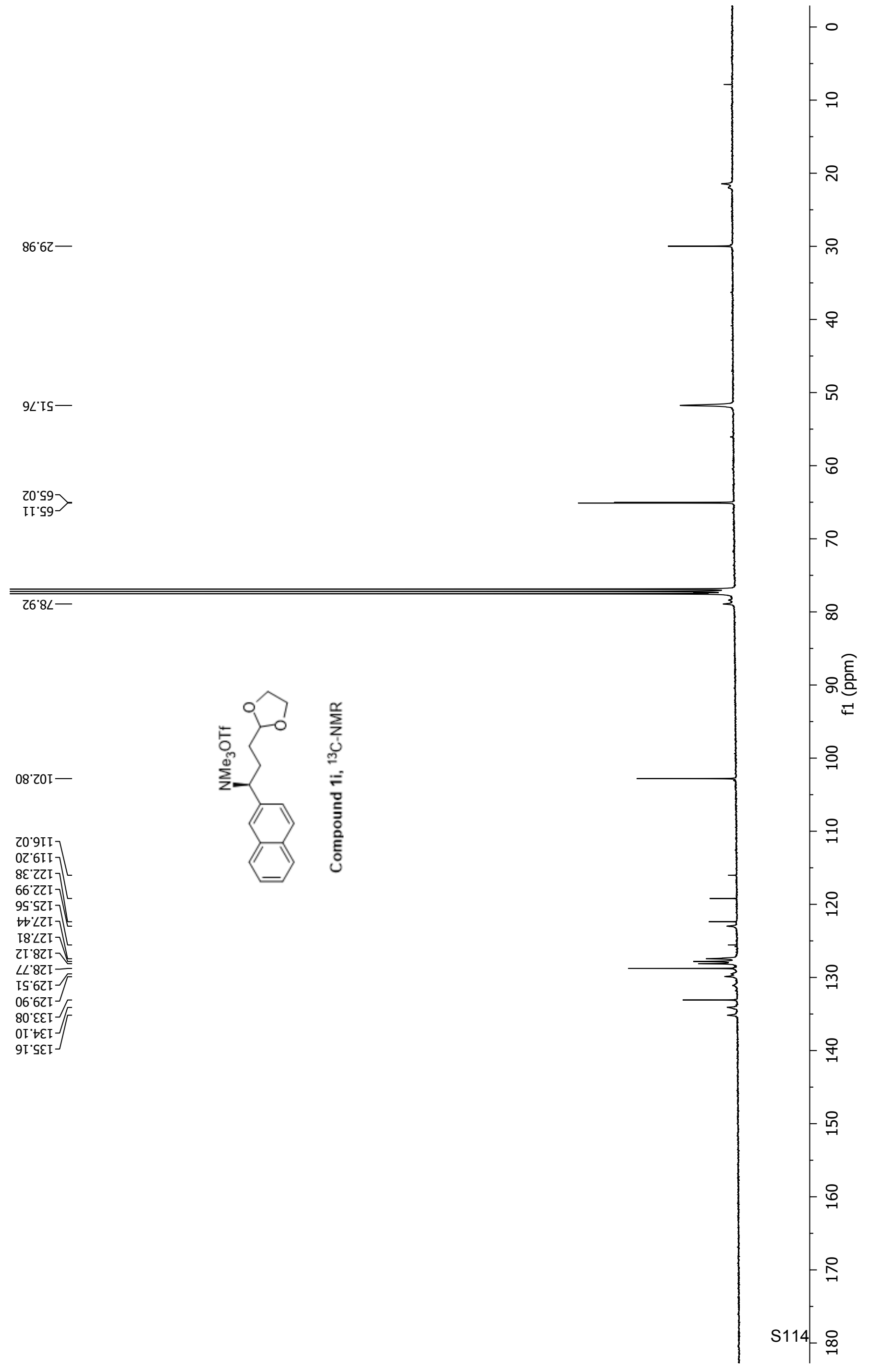


(mdd) If

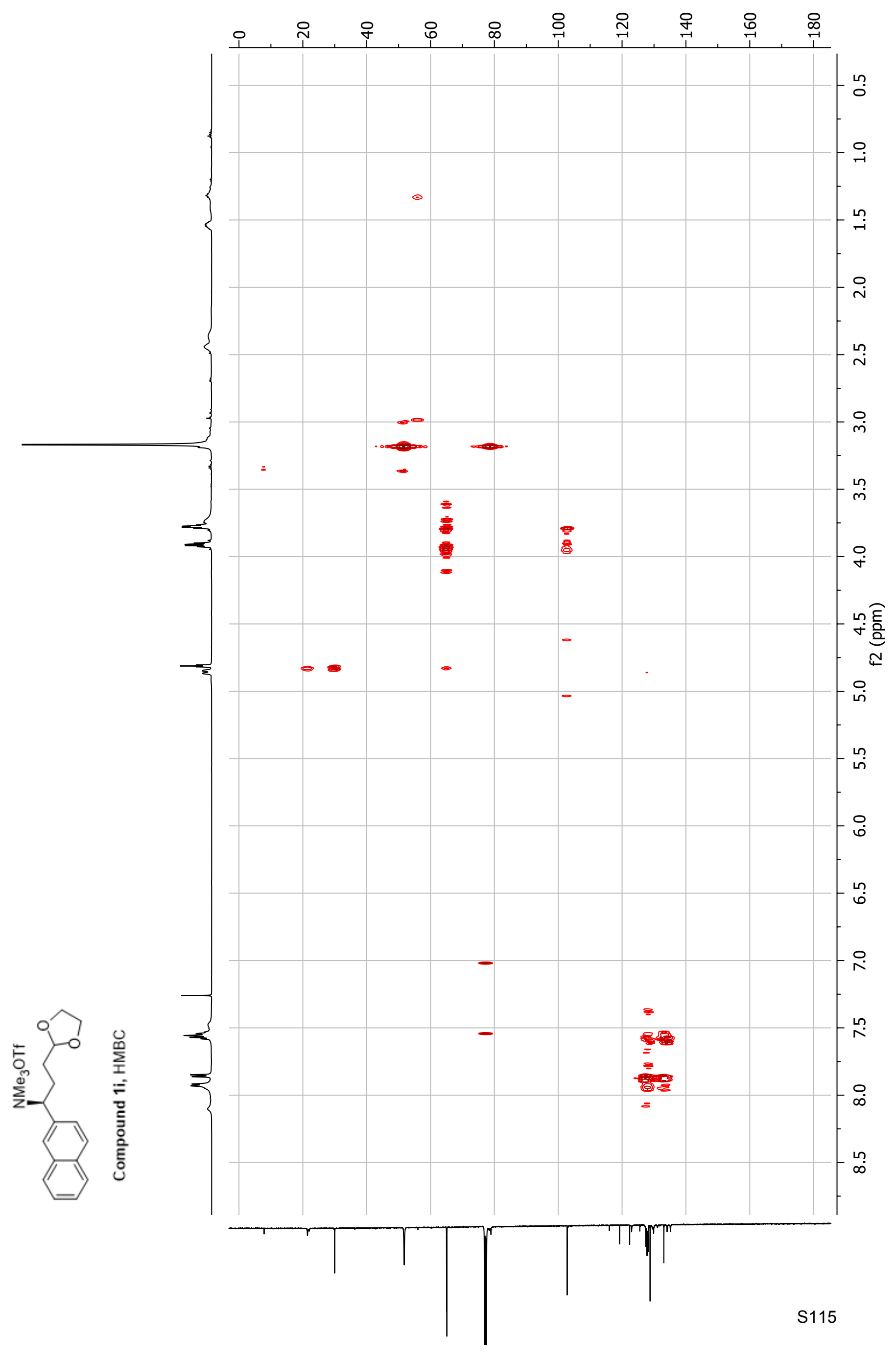




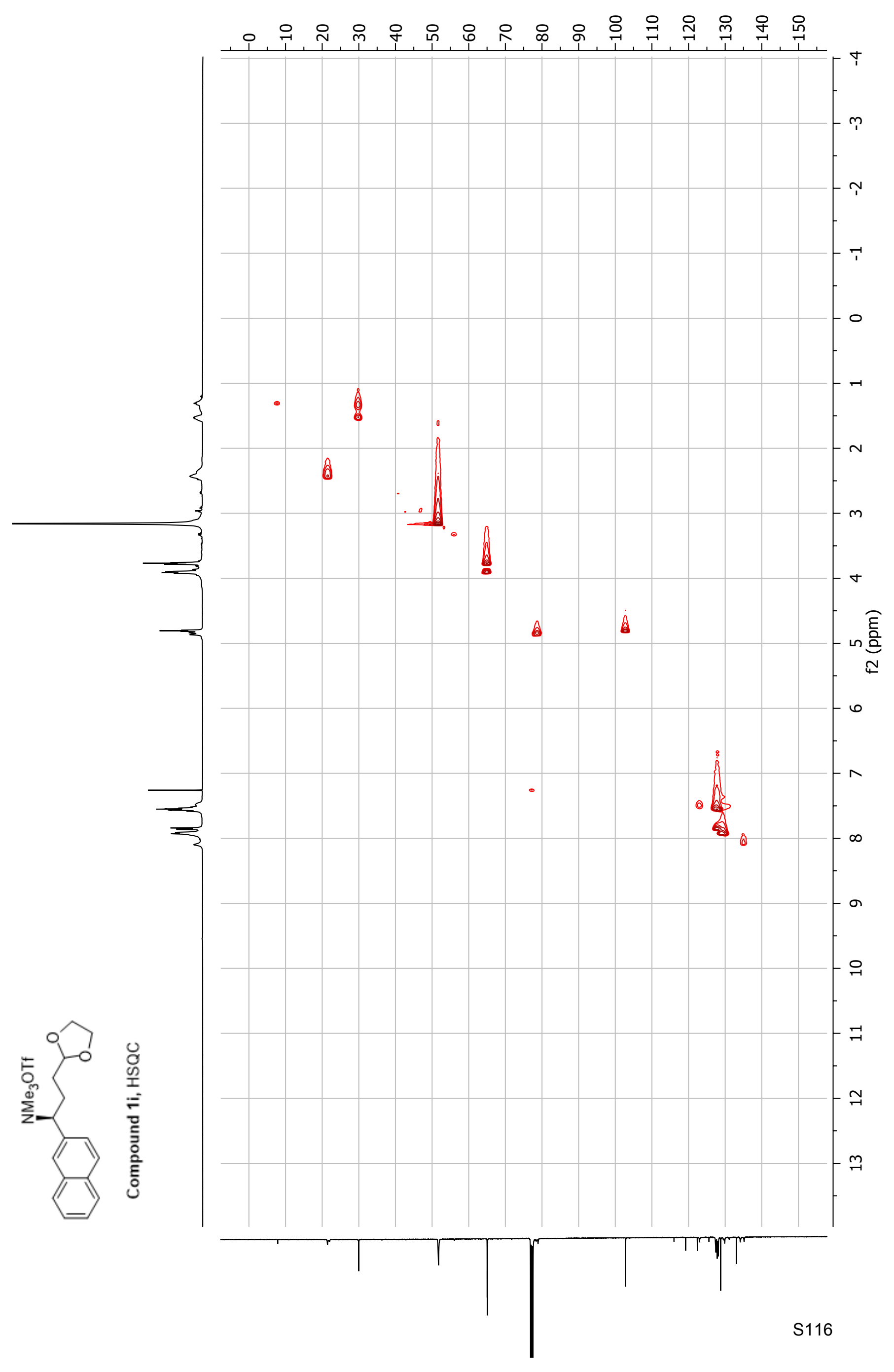




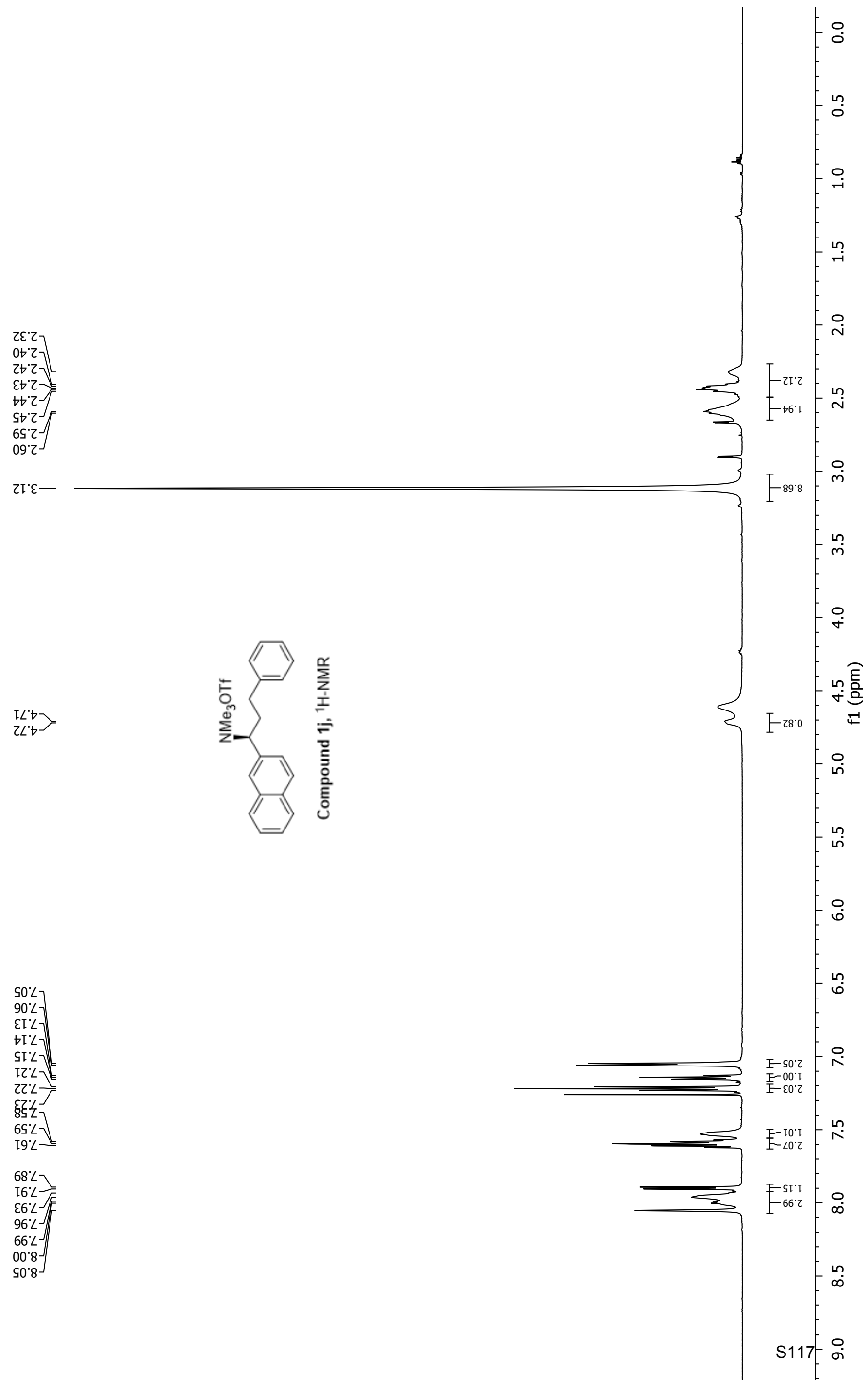



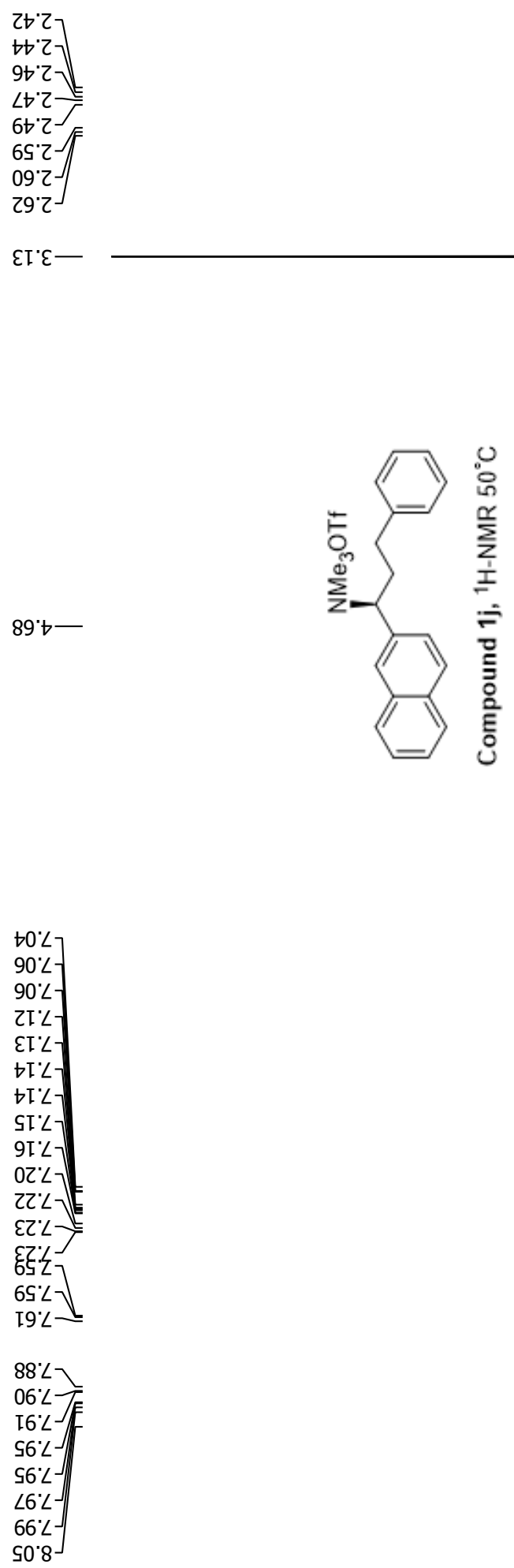

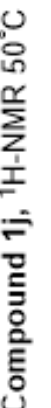

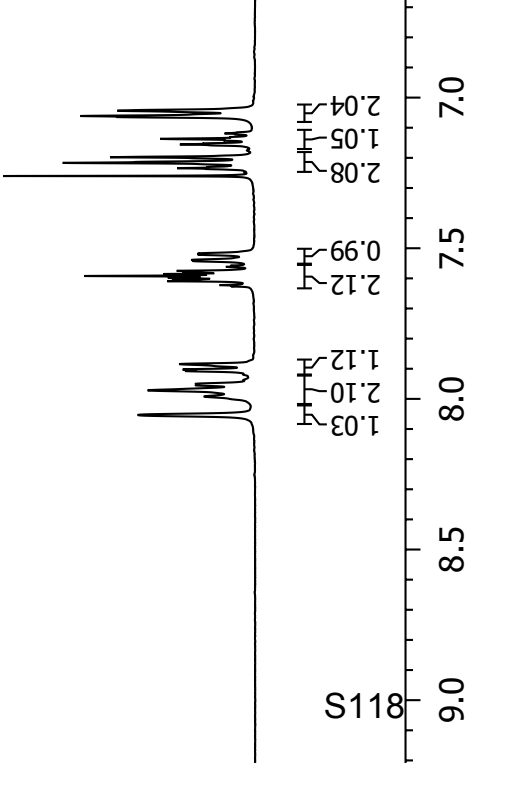



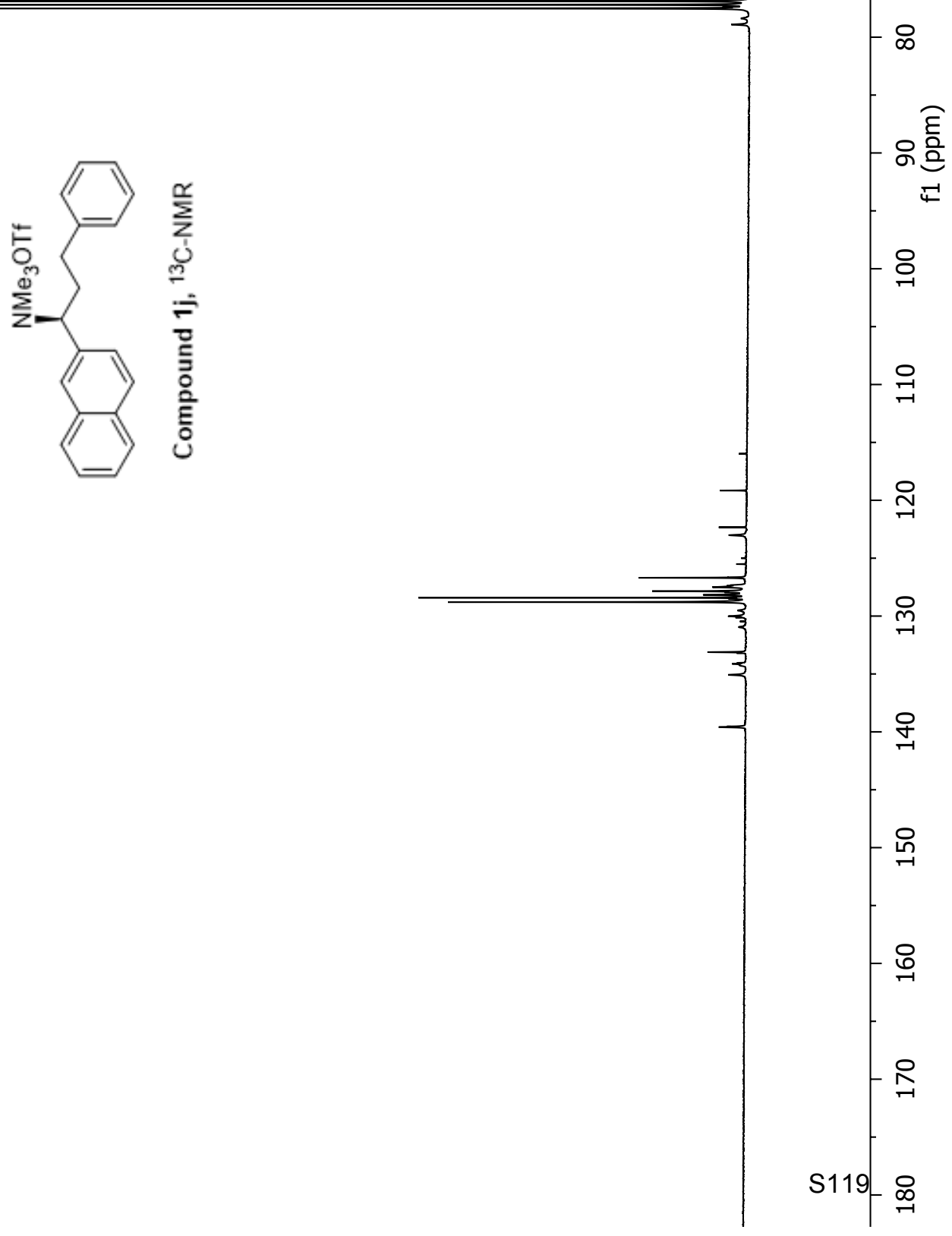
(mdd) If

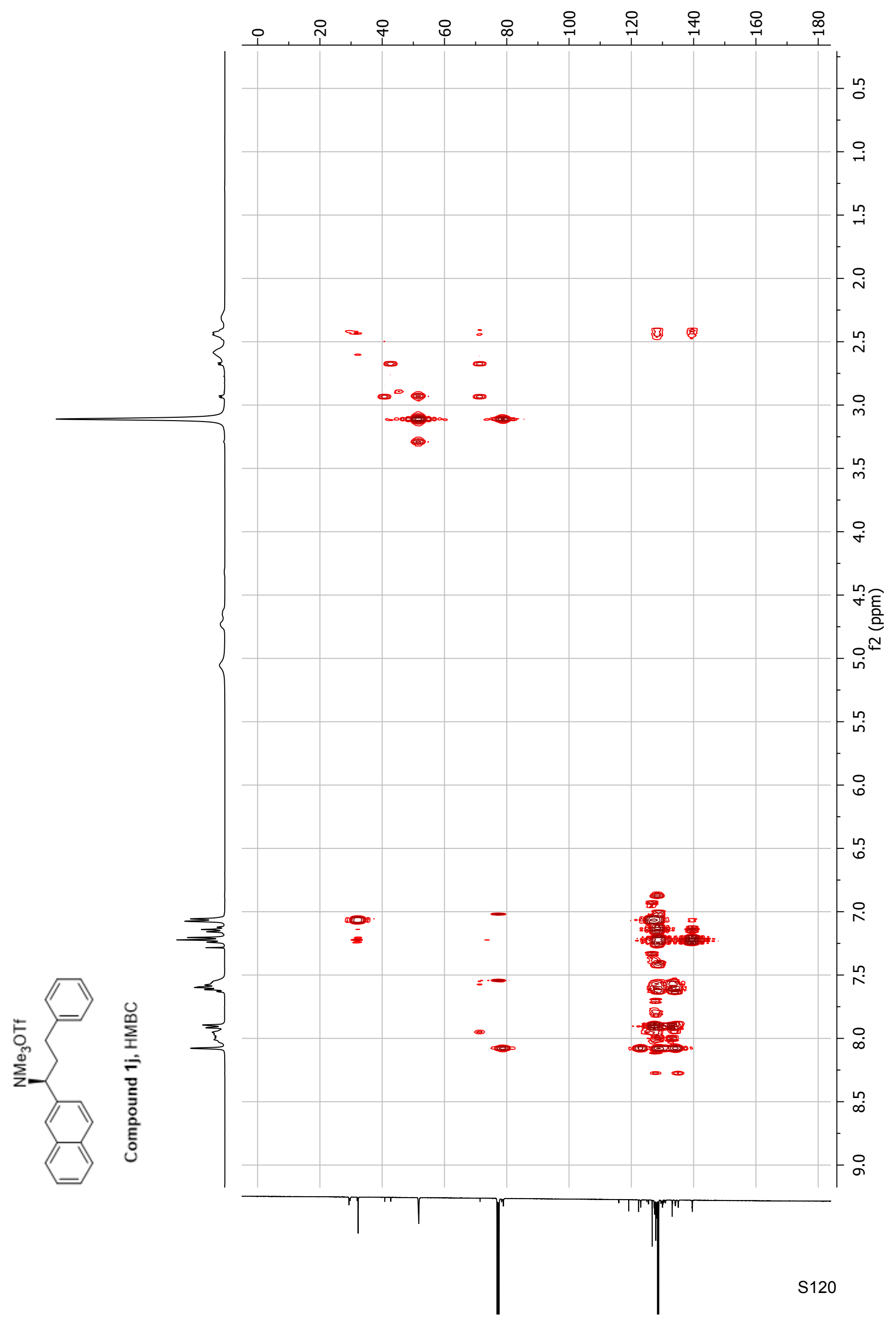




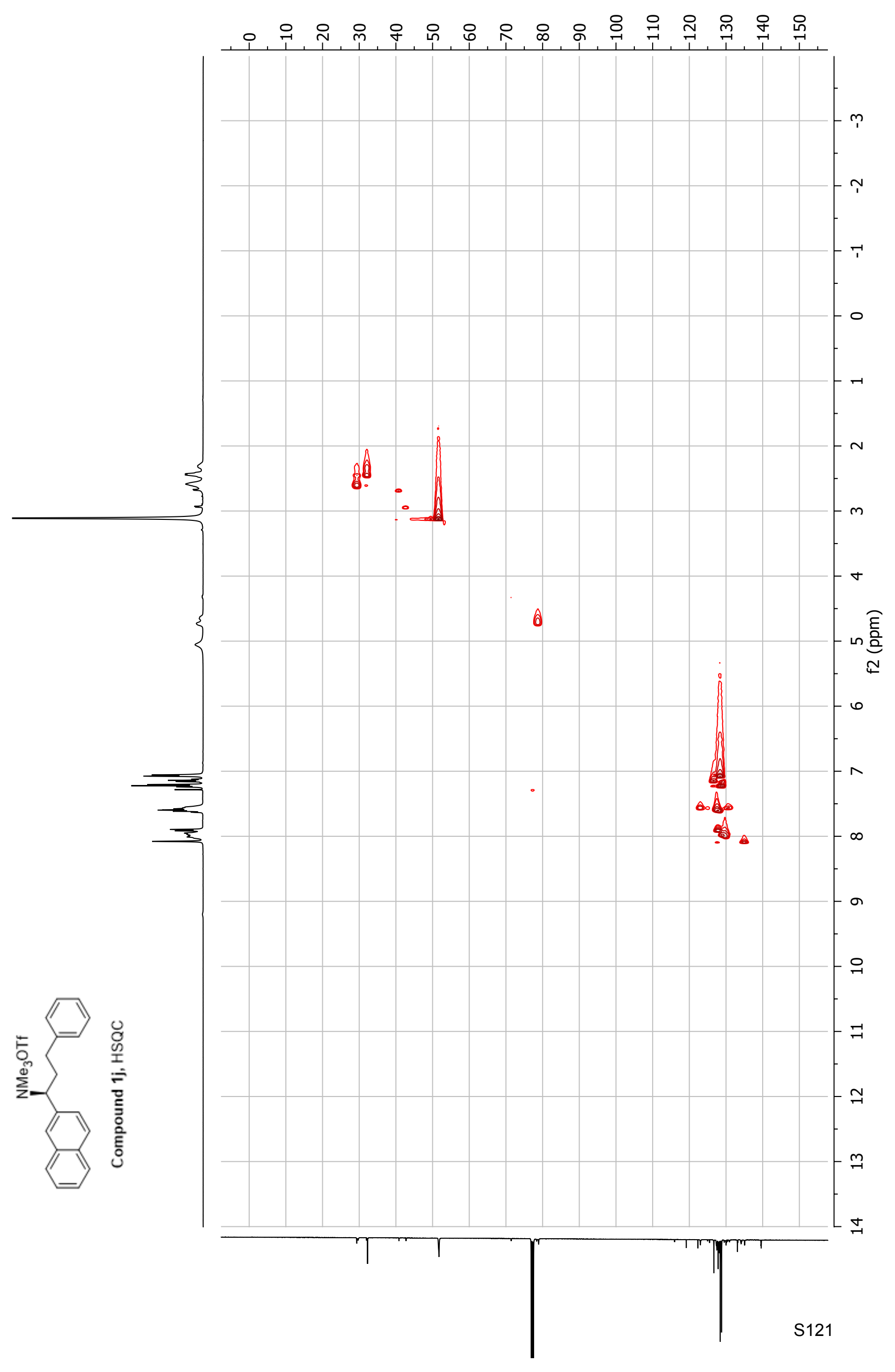




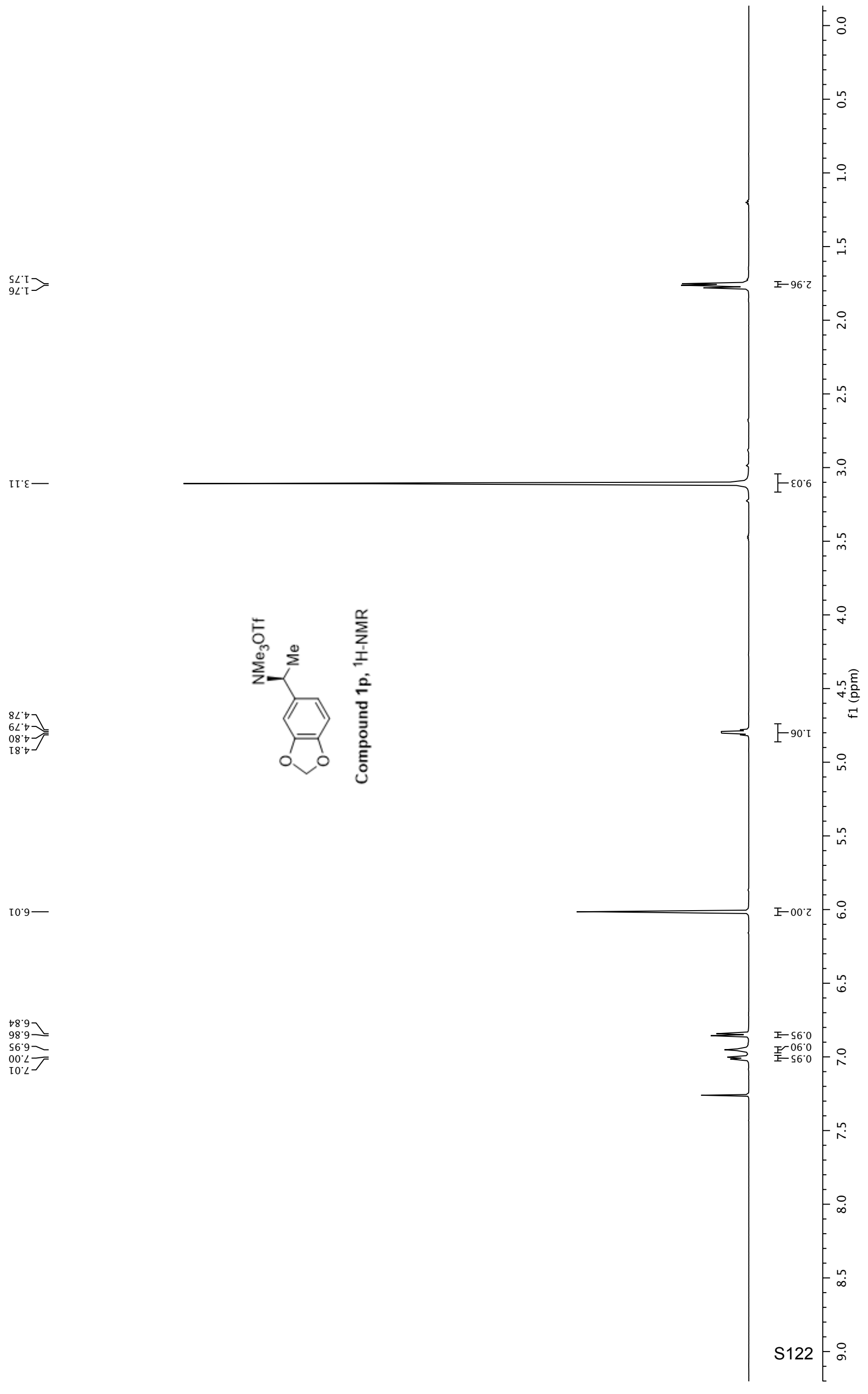




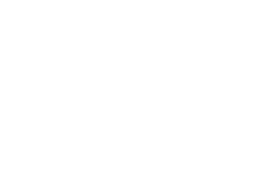

En

$\angle 0^{\circ} \sqcup \angle$

$66.9 L-$

$\angle 0^{\circ} 201-$

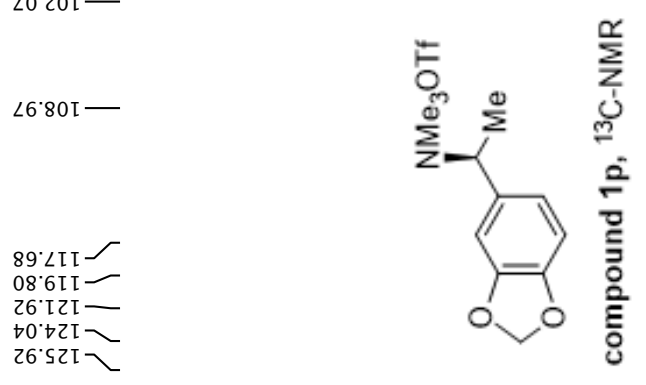




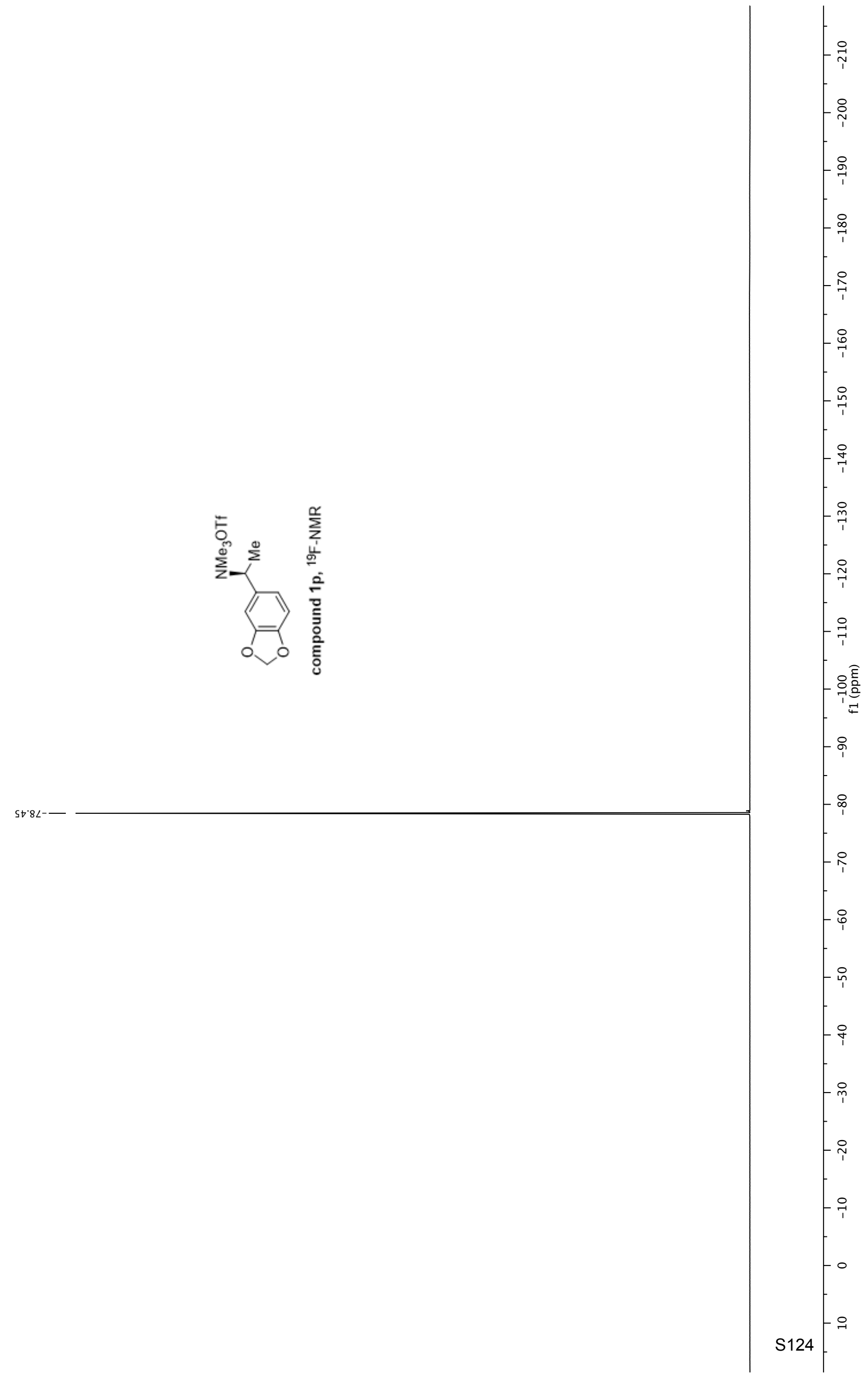




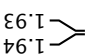

$9 I^{\prime} \varepsilon-$

$82^{\circ} \varsigma$

$62^{\circ} \mathrm{s}$

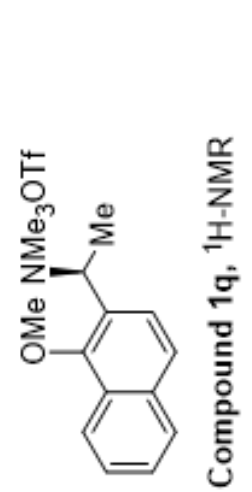

$00{ }^{\circ}$

$6 t^{\circ} \angle 7$

IS.L]

$8 \mathrm{~S}^{\circ} \mathrm{L}$
$6 \mathrm{~S}^{\circ} \mathrm{L}$

$6 S^{\circ} \angle-$

$09 . \angle$

$\left[9^{\circ} \angle\right.$

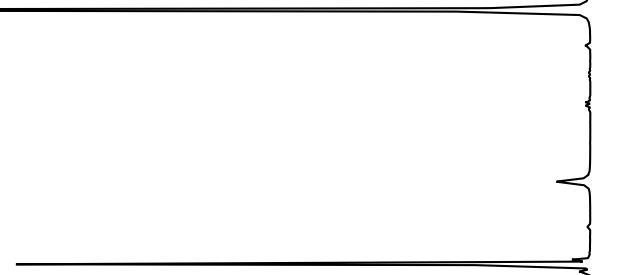


$8 \varepsilon^{\circ} \mathrm{SI}$

$9 \varepsilon \cdot I S$

$\left.\begin{array}{l}9 \varepsilon \cdot \text { 'L } \\ 8 \varepsilon \text {.IS }\end{array}\right\}$

$06 \cdot \varepsilon 9$

$\rightarrow I<9-$

[ $\angle \cdot \angle I T$

ह8.6 [ [ ]

$\rightarrow 6^{\circ} 02[]$

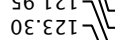

$\left.\begin{array}{ll}0 \circ \\ 0 \varepsilon\end{array}\right]$

$6 \varepsilon^{\circ} \forall Z I-$
$85^{\circ} \varsigma 2 \mathrm{I}$

85. $\angle 2 I-$

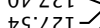

งह.8Z[

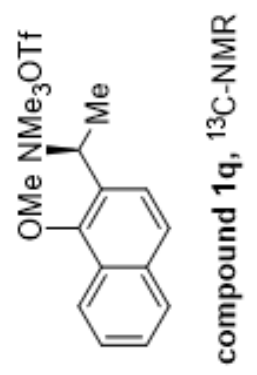

ธ6. ऽะโ -
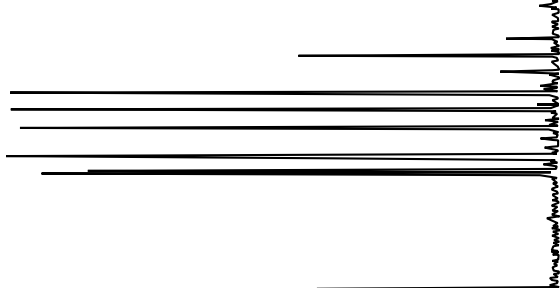


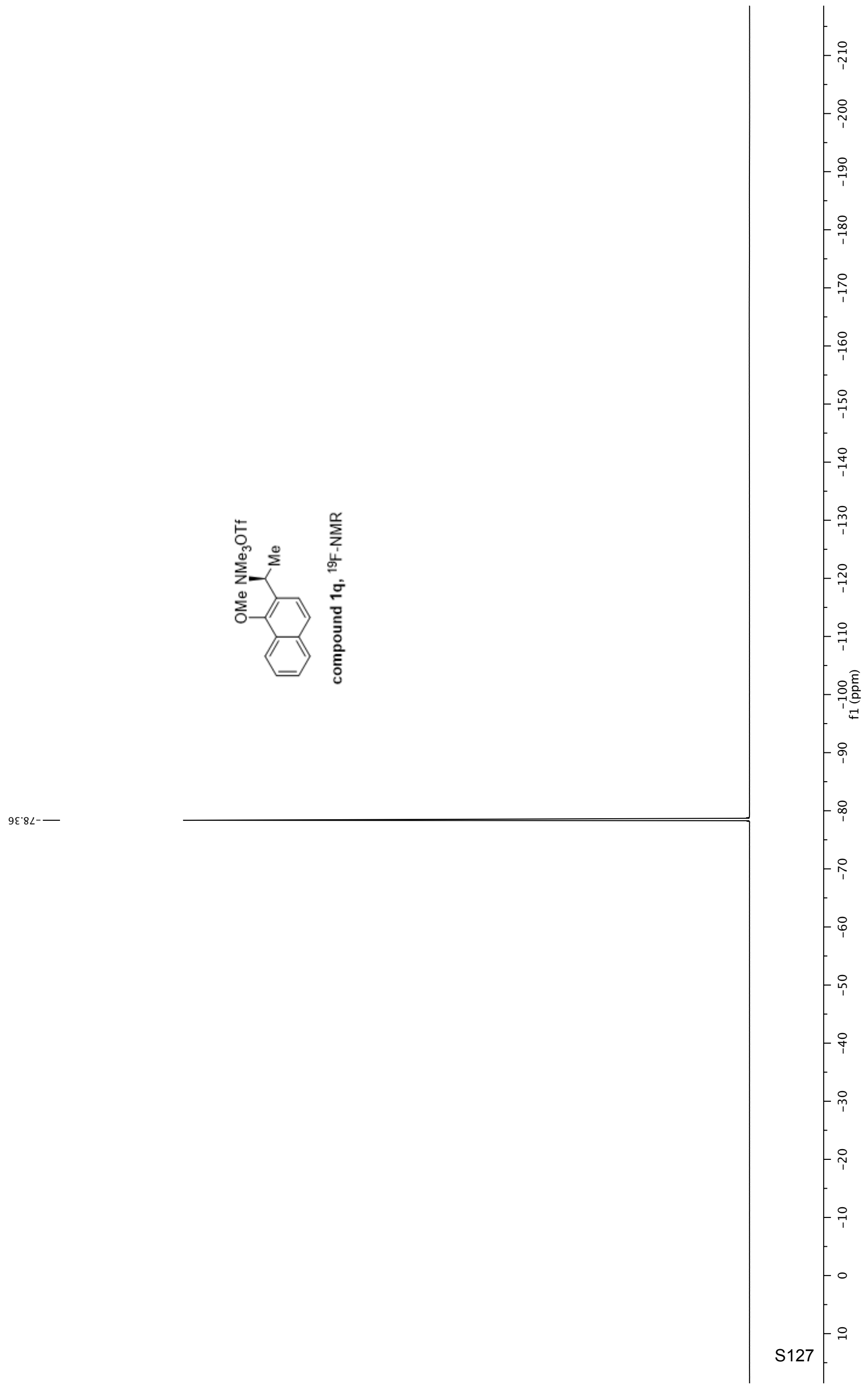


Compound 3a, racemic (254 nm)

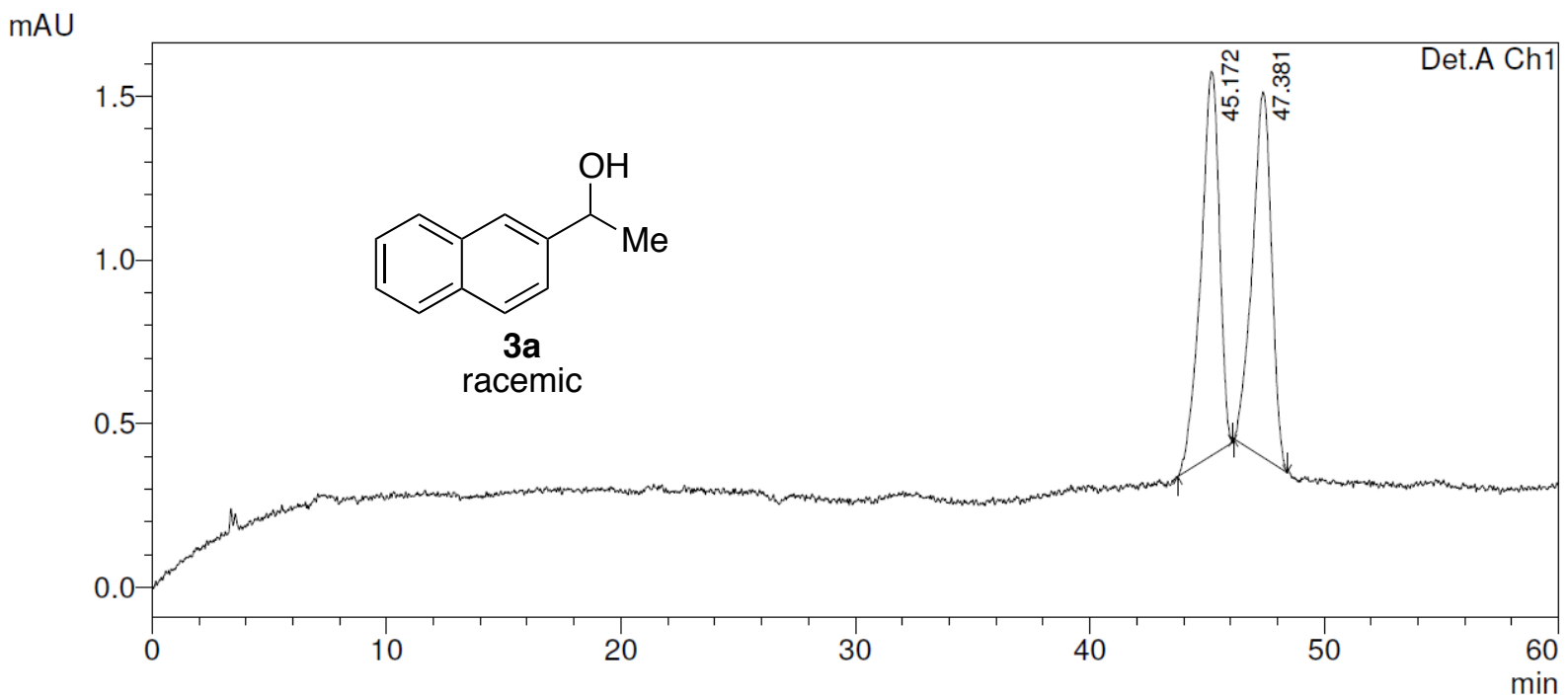

\begin{tabular}{|r|r|r|r|r|r|}
\hline \multicolumn{1}{|c|}{ Peak\# } & Ret. Time & \multicolumn{1}{c|}{ Area } & \multicolumn{1}{c|}{ Height } & \multicolumn{1}{c|}{ Area \% } & Height \% \\
\hline 1 & 45.172 & 64627 & 1174 & 50.417 & 51.245 \\
\hline 2 & 47.381 & 63558 & 1117 & 49.583 & 48.755 \\
\hline Total & & 128185 & 2292 & 100.000 & 100.000 \\
\hline
\end{tabular}

Compound 3a, 99\% ee (254 nm)

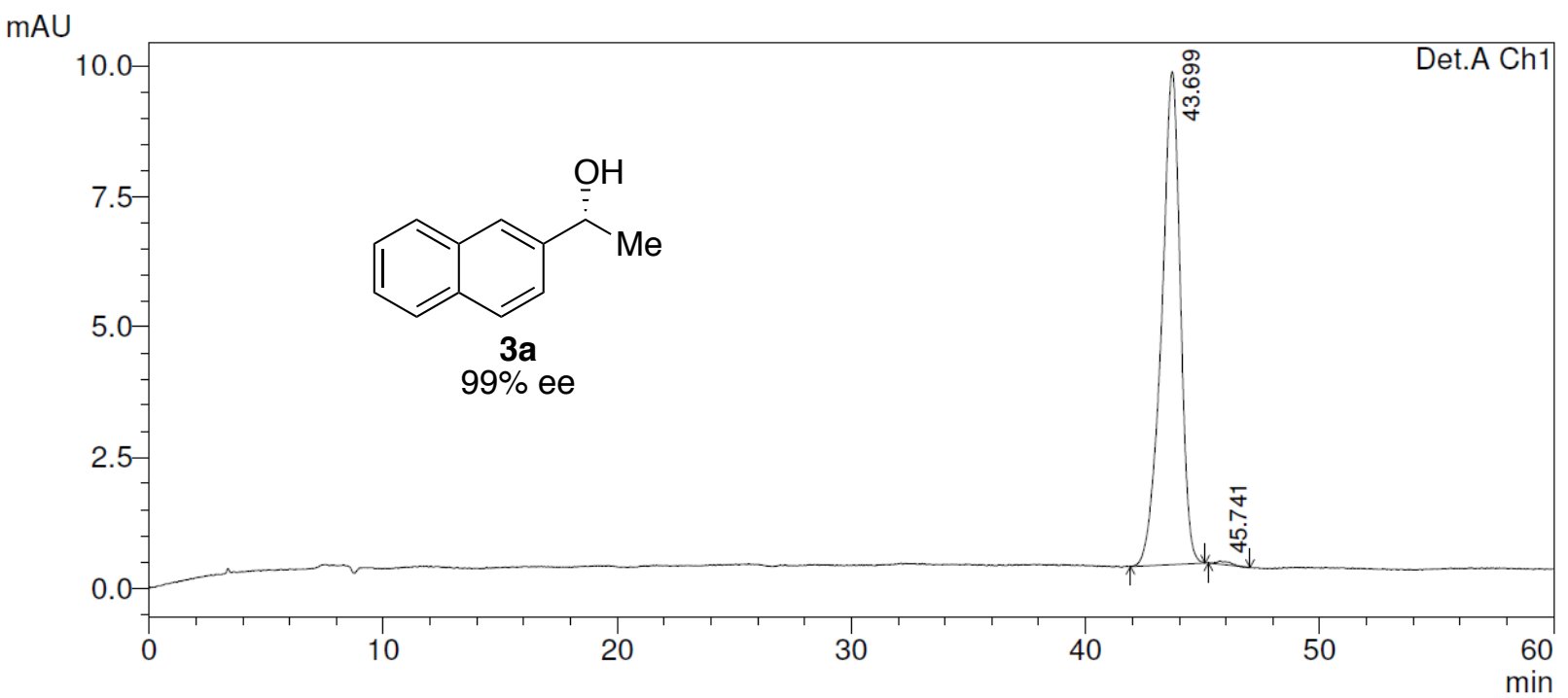

\begin{tabular}{|r|r|r|r|r|r|}
\hline \multicolumn{1}{|c|}{ Peak\# } & Ret. Time & \multicolumn{1}{c|}{ Area } & \multicolumn{1}{|c|}{ Height } & Area \% & Height \% \\
\hline 1 & 43.699 & 524955 & 9434 & 99.543 & 99.356 \\
\hline 2 & 45.741 & 2408 & 61 & 0.457 & 0.644 \\
\hline Total & & 527362 & 9495 & 100.000 & 100.000 \\
\hline
\end{tabular}


Compound 3b, racemic ( $254 \mathrm{~nm}$ )

mAU

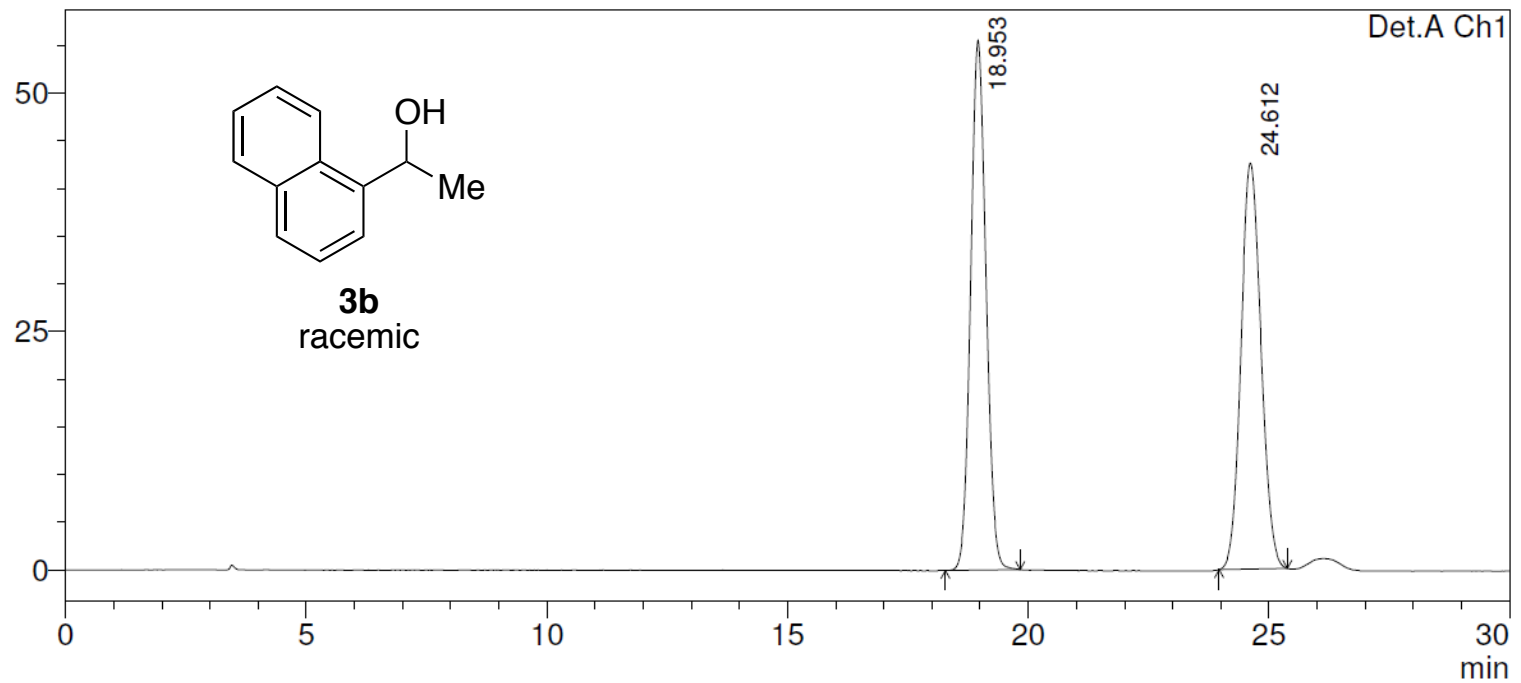

\begin{tabular}{|r|r|r|r|r|r|}
\hline \multicolumn{1}{|c|}{ Peak\# } & Ret. Time & Area & Height & Area \% & \multicolumn{1}{c|}{ Height \% } \\
\hline 1 & 18.953 & 1239231 & 55611 & 50.146 & 56.632 \\
\hline 2 & 24.612 & 1232017 & 42586 & 49.854 & 43.368 \\
\hline Total & & 2471248 & 98197 & 100.000 & 100.000 \\
\hline
\end{tabular}

Compound 3 b, 92\% ee ( $254 \mathrm{~nm}$ )

mAU

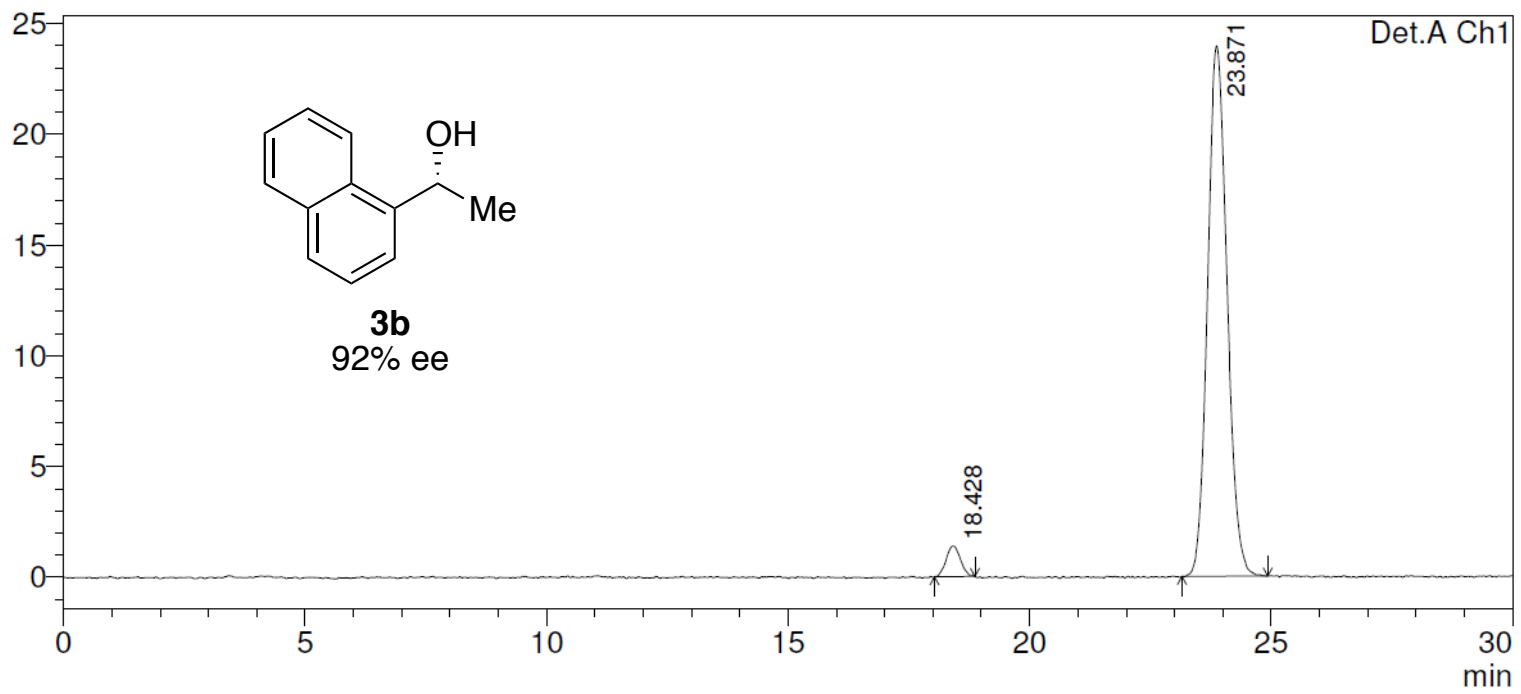

\begin{tabular}{|r|r|r|r|r|r|}
\hline \multicolumn{1}{|c|}{ Peak\# } & Ret. Time & \multicolumn{1}{c|}{ Area } & Height & Area \% & Height \% \\
\hline 1 & 18.428 & 28226 & 1362 & 4.034 & 5.381 \\
\hline 2 & 23.871 & 671567 & 23948 & 95.966 & 94.619 \\
\hline Total & & 699793 & 25310 & 100.000 & 100.000 \\
\hline
\end{tabular}


Compound 3c, racemic (254 nm)

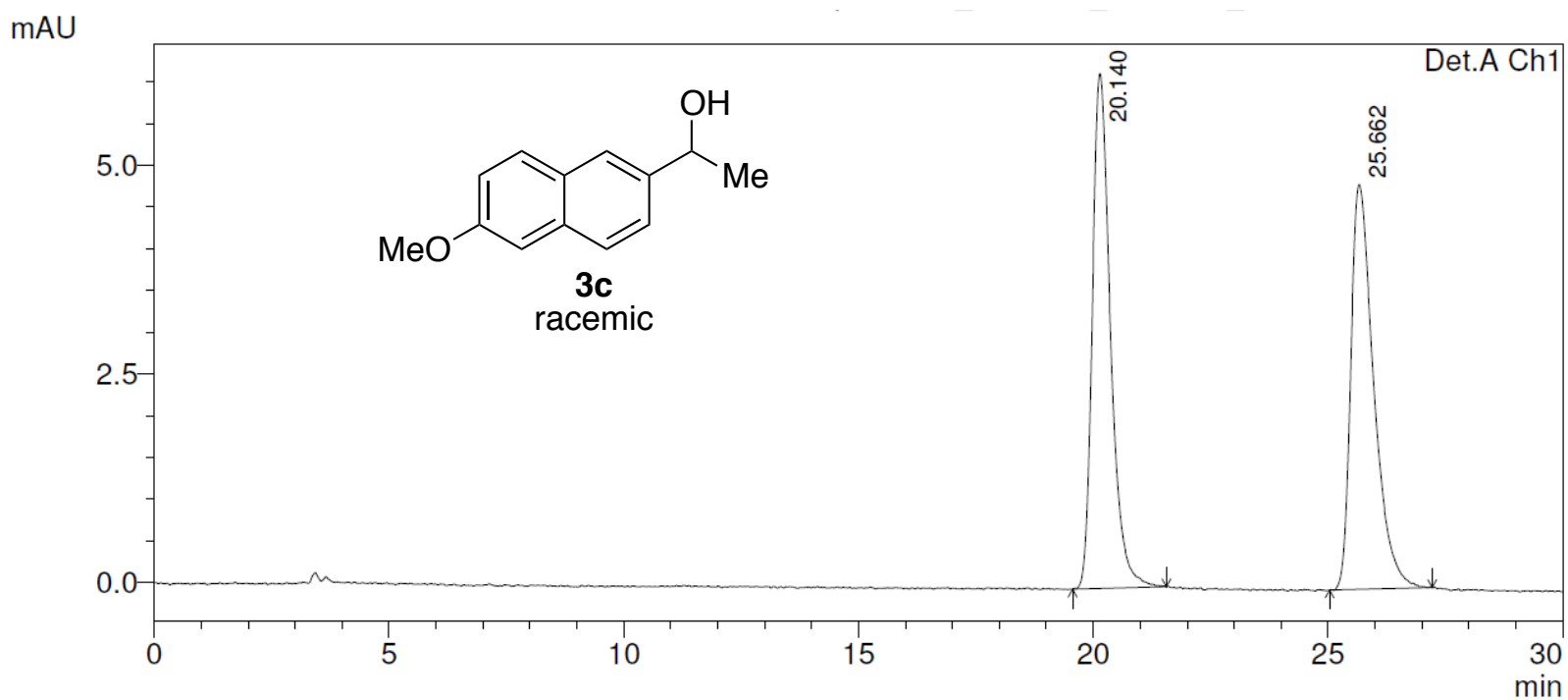

\begin{tabular}{|r|r|r|r|r|r|}
\hline \multicolumn{1}{|c|}{ Peak\# } & Ret. Time & \multicolumn{1}{c|}{ Area } & Height & Area \% & Height \% \\
\hline 1 & 20.140 & 165137 & 6165 & 50.153 & 55.982 \\
\hline 2 & 25.662 & 164128 & 4848 & 49.847 & 44.018 \\
\hline Total & & 329266 & 11013 & 100.000 & 100.000 \\
\hline
\end{tabular}

Compound 3c, 99\% ee (254 nm)

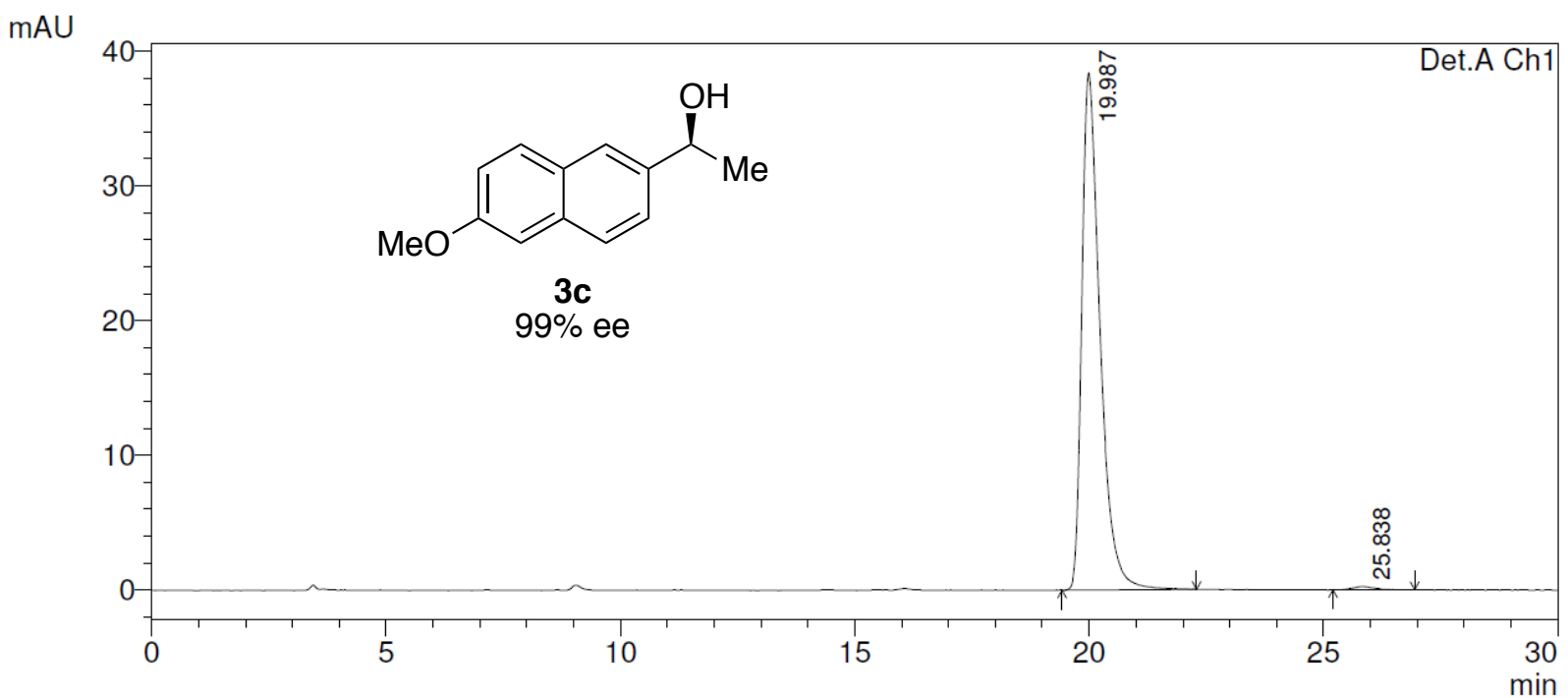

\begin{tabular}{|r|r|r|r|r|r|}
\hline \multicolumn{1}{|c|}{ Peak\# } & Ret. Time & \multicolumn{1}{c|}{ Area } & Height & Area \% & Height \% \\
\hline 1 & 19.987 & 1037849 & 38360 & 99.309 & 99.372 \\
\hline 2 & 25.838 & 7220 & 242 & 0.691 & 0.628 \\
\hline Total & & 1045069 & 38602 & 100.000 & 100.000 \\
\hline
\end{tabular}


Compound 3d, racemic (254 nm)

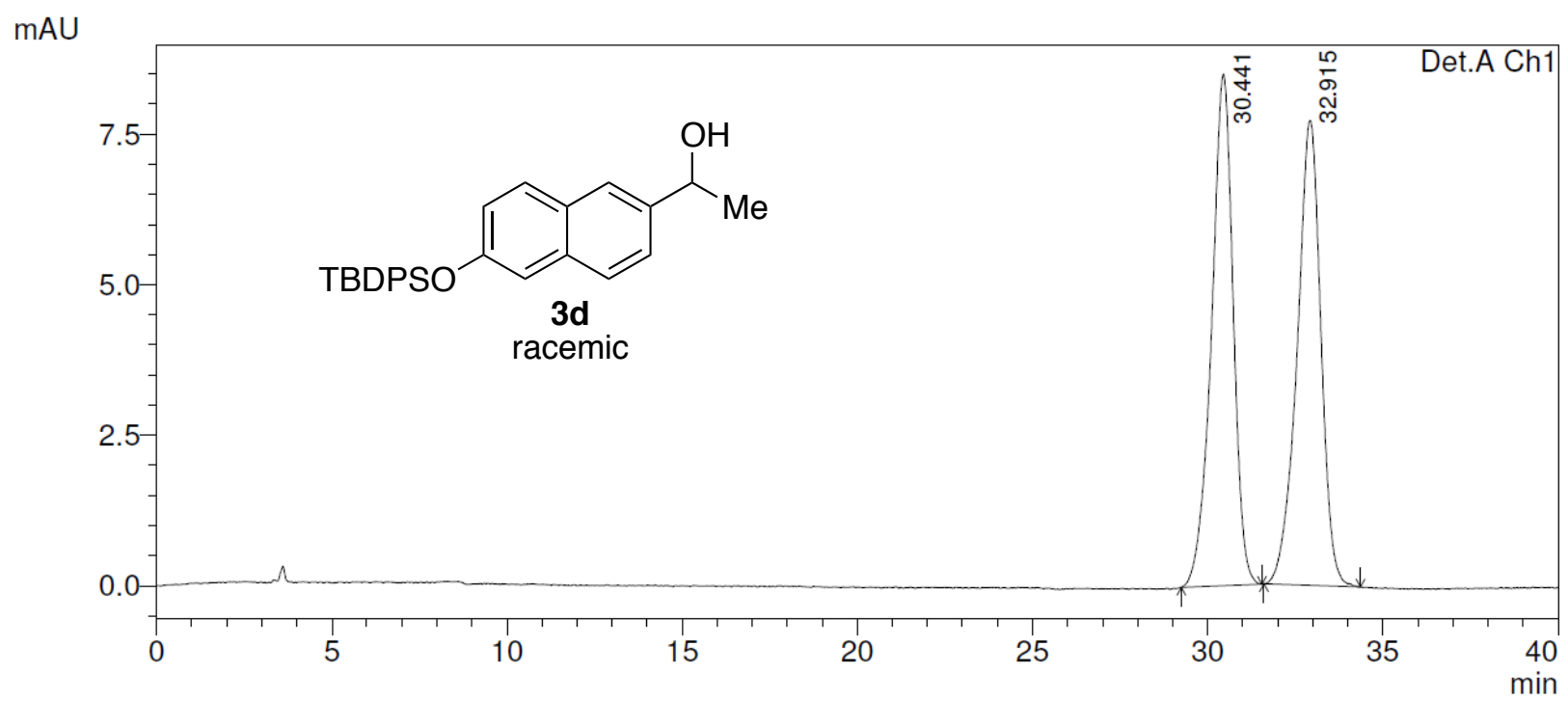

\begin{tabular}{|r|r|r|r|r|r|}
\hline Peak\# & Ret. Time & Area & Height & Area \% & Height \% \\
\hline 1 & 30.441 & 364152 & 8496 & 49.958 & 52.369 \\
\hline 2 & 32.915 & 364762 & 7727 & 50.042 & 47.631 \\
\hline Total & & 728913 & 16223 & 100.000 & 100.000 \\
\hline
\end{tabular}

Compound 3d, 92\% ee (254 nm)

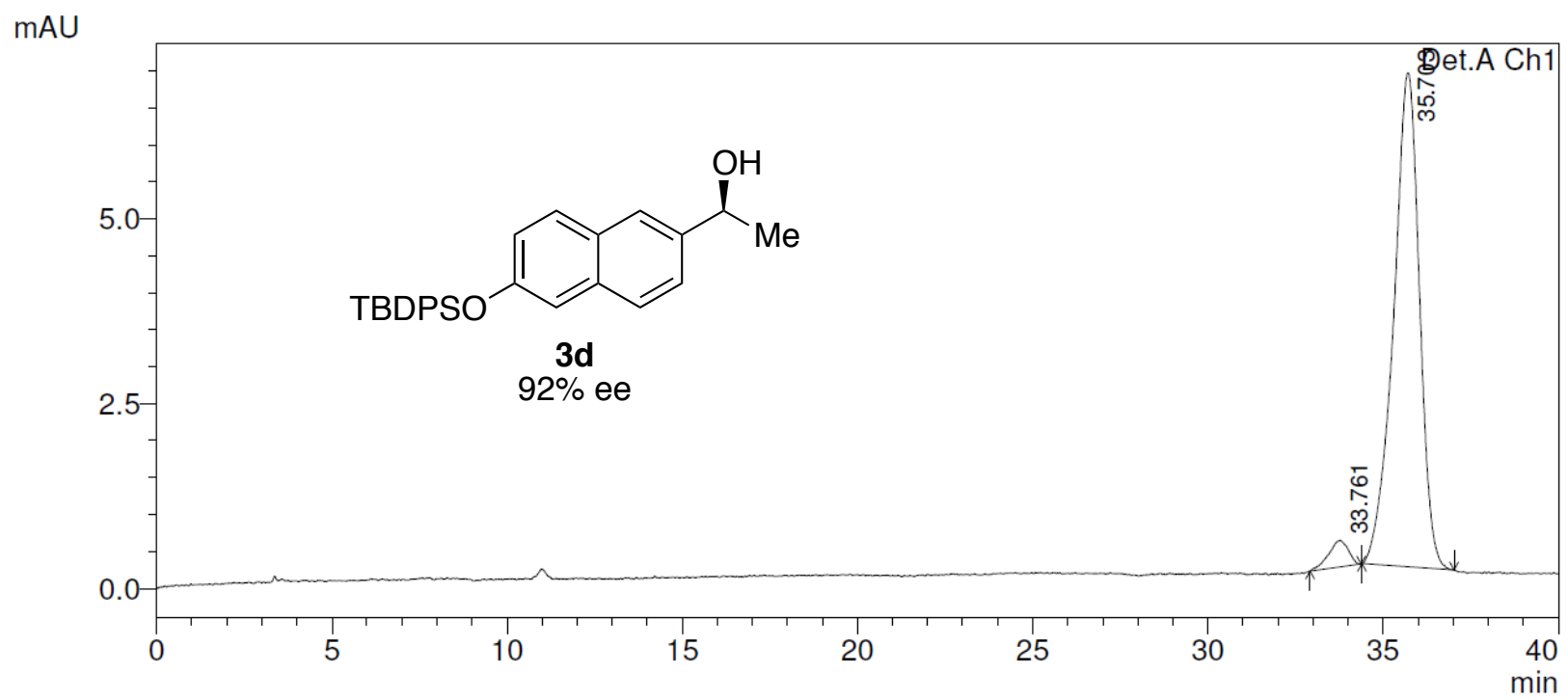

\begin{tabular}{|r|r|r|r|r|r|}
\hline \multicolumn{1}{|c|}{ Peak\# } & Ret. Time & \multicolumn{1}{c|}{ Area } & \multicolumn{1}{c|}{ Height } & Area \% & Height \% \\
\hline 1 & 33.761 & 14125 & 358 & 3.958 & 5.084 \\
\hline 2 & 35.703 & 342763 & 6682 & 96.042 & 94.916 \\
\hline Total & & 356888 & 7040 & 100.000 & 100.000 \\
\hline
\end{tabular}


Compound 3e, racemic (254 nm)

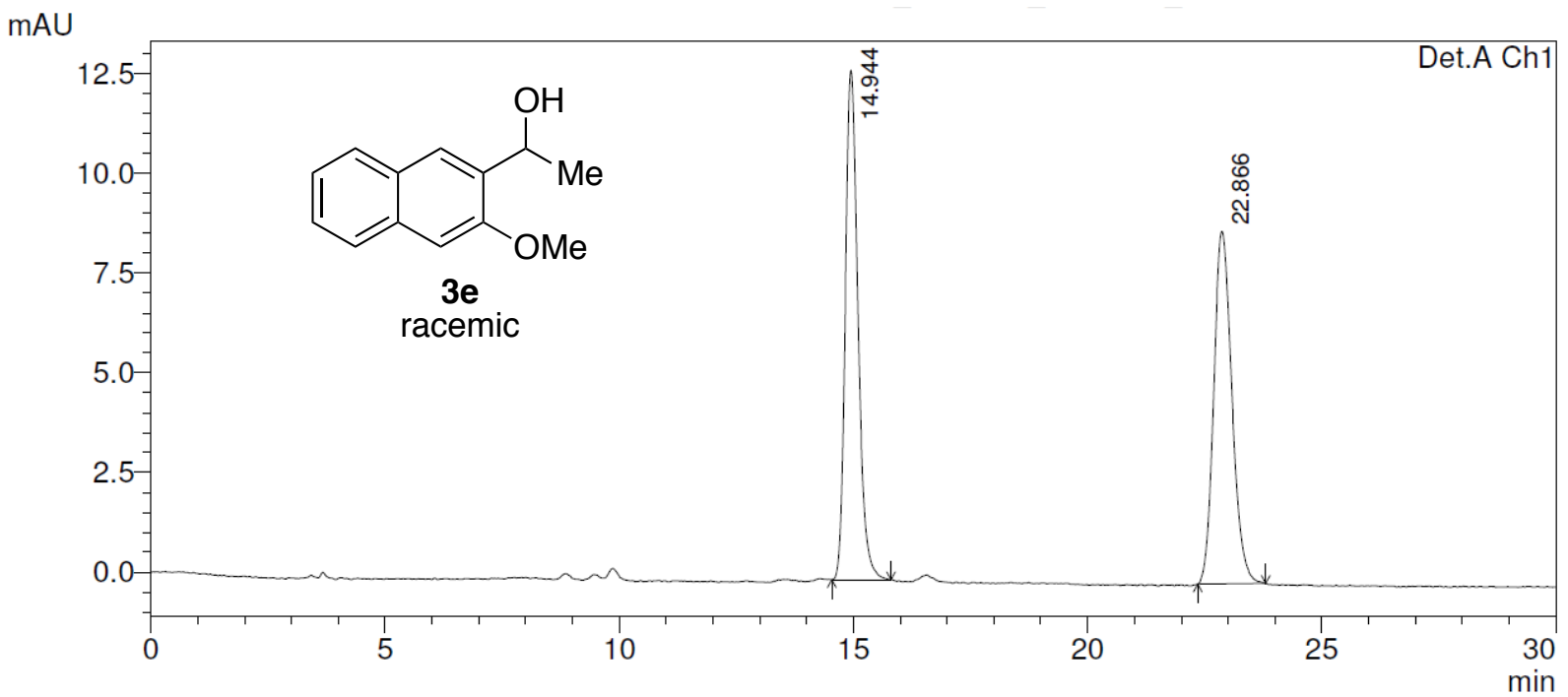

\begin{tabular}{|r|r|r|r|r|r|}
\hline \multicolumn{1}{|c|}{ Peak\# } & Ret. Time & Area & Height & \multicolumn{1}{|c|}{ Area \% } & \multicolumn{1}{c|}{ Height \% } \\
\hline 1 & 14.944 & 239370 & 12773 & 50.150 & 59.107 \\
\hline 2 & 22.866 & 237935 & 8837 & 49.850 & 40.893 \\
\hline Total & & 477305 & 21610 & 100.000 & 100.000 \\
\hline
\end{tabular}

Compound 3e, 95\% ee (254 nm)

mAU

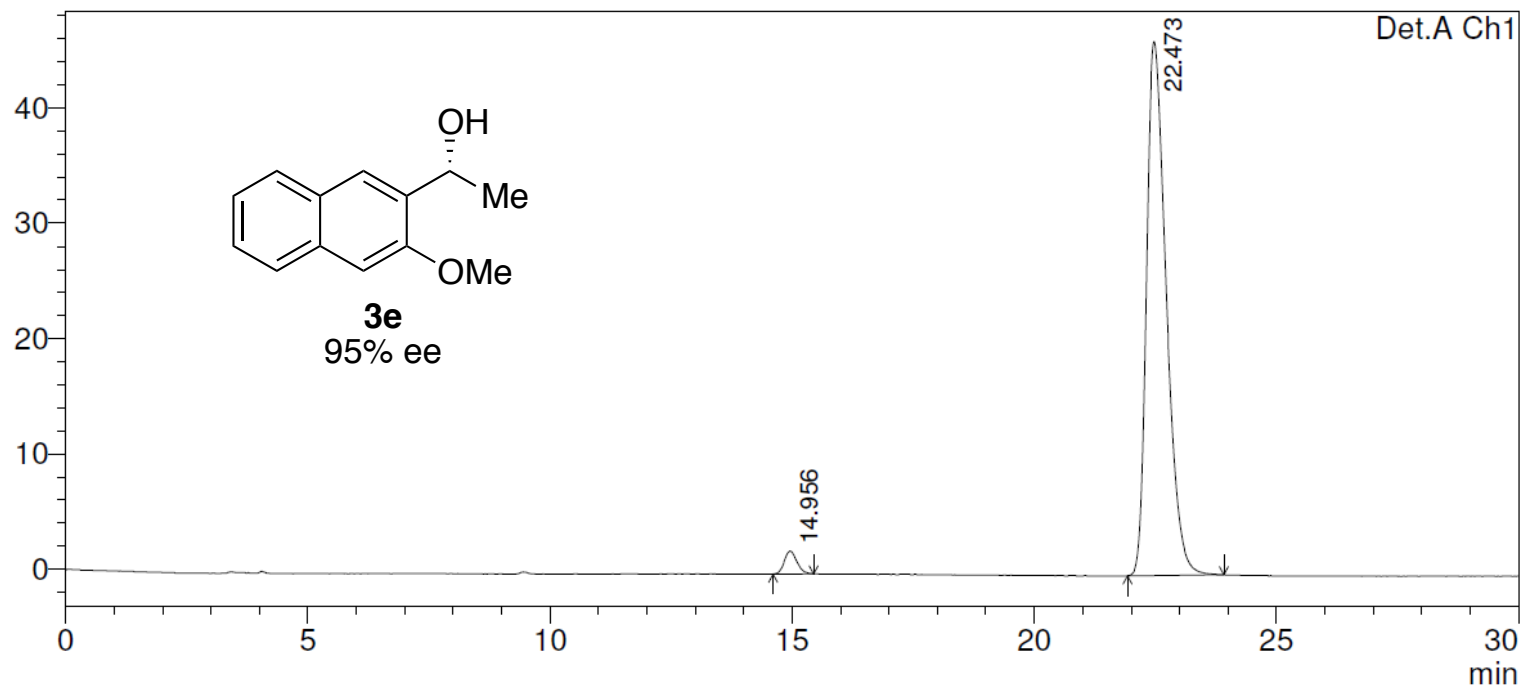

\begin{tabular}{|r|r|r|r|r|r|}
\hline \multicolumn{1}{|c|}{ Peak\# } & Ret. Time & \multicolumn{1}{c|}{ Area } & \multicolumn{1}{c|}{ Height } & \multicolumn{1}{c|}{ Area \% } & \multicolumn{1}{c|}{ Height \% } \\
\hline 1 & 14.956 & 35963 & 1982 & 2.675 & 4.104 \\
\hline 2 & 22.473 & 1308675 & 46315 & 97.325 & 95.896 \\
\hline Total & & 1344638 & 48297 & 100.000 & 100.000 \\
\hline
\end{tabular}


Compound 3f, racemic (254 nm)

mAU

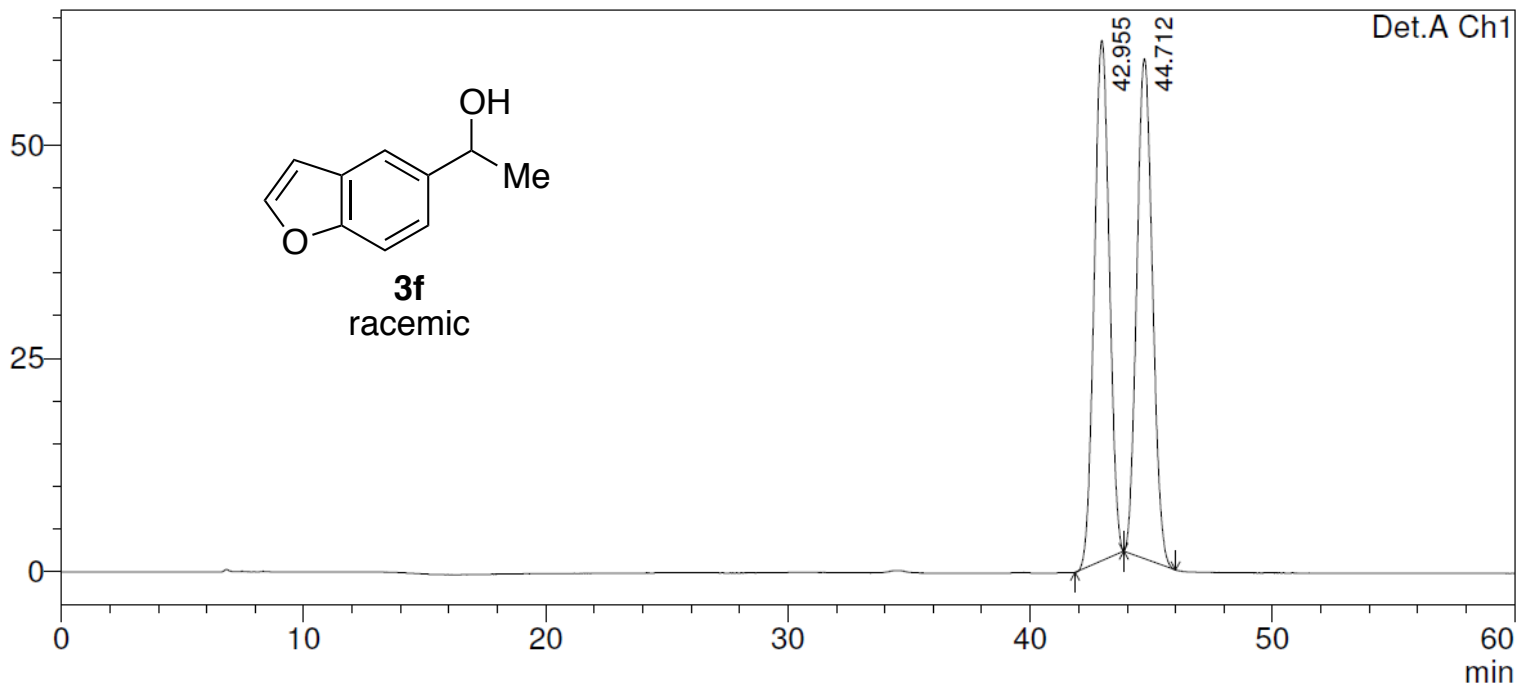

\begin{tabular}{|r|r|r|r|r|r|}
\hline \multicolumn{1}{|c|}{ Peak\# } & Ret. Time & Area & Height & Area \% & Height \% \\
\hline 1 & 42.955 & 2640838 & 61031 & 49.950 & 51.012 \\
\hline 2 & 44.712 & 2646127 & 58610 & 50.050 & 48.988 \\
\hline Total & & 5286965 & 119641 & 100.000 & 100.000 \\
\hline
\end{tabular}

Compound 3f, 98\% ee (254 nm)

mAU

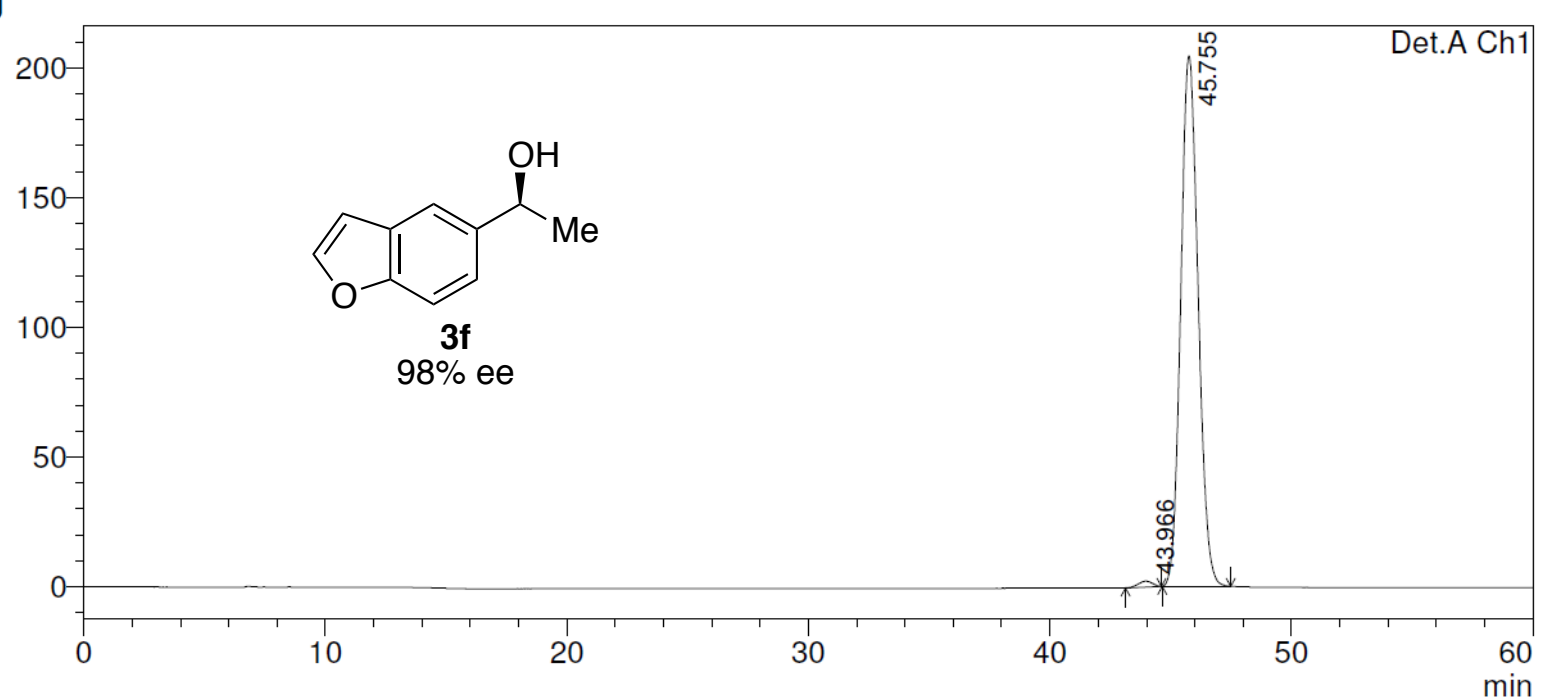

\begin{tabular}{|r|r|r|r|r|r|}
\hline \multicolumn{1}{|c|}{ Peak\# } & Ret. Time & \multicolumn{1}{c|}{ Area } & Height & Area \% & Height \% \\
\hline 1 & 43.966 & 90232 & 2245 & 0.873 & 1.086 \\
\hline 2 & 45.755 & 10242584 & 204407 & 99.127 & 98.914 \\
\hline Total & & 10332816 & 206652 & 100.000 & 100.000 \\
\hline
\end{tabular}


Compound 3g, racemic (254 nm)

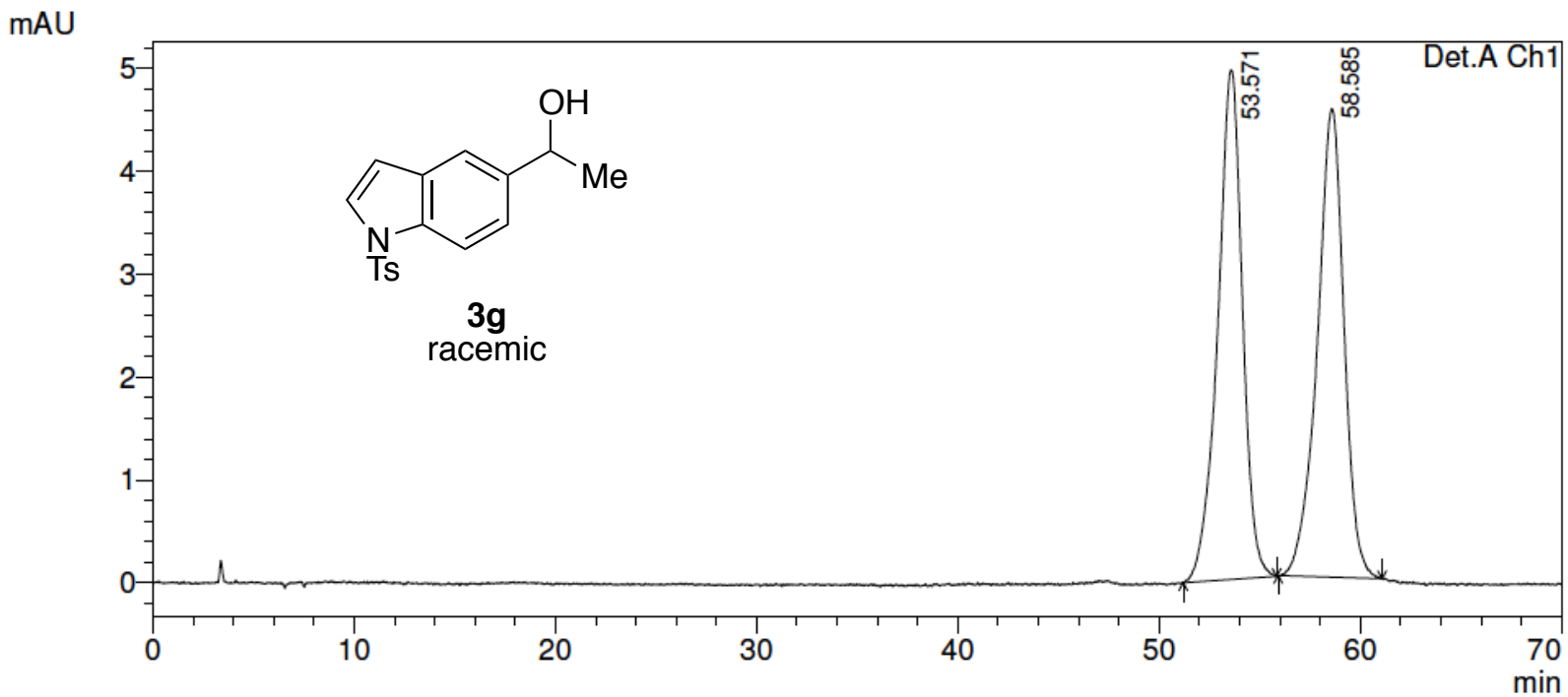

\begin{tabular}{|r|r|r|r|r|r|}
\hline Peak\# & Ret. Time & \multicolumn{1}{c|}{ Area } & \multicolumn{1}{c|}{ Height } & Area \% & Height \% \\
\hline 1 & 53.571 & 422898 & 4957 & 50.129 & 52.089 \\
\hline 2 & 58.585 & 420722 & 4559 & 49.871 & 47.911 \\
\hline Total & & 843620 & 9516 & 100.000 & 100.000 \\
\hline
\end{tabular}

Compound 3g, 96\% ee (254 nm)

mAU

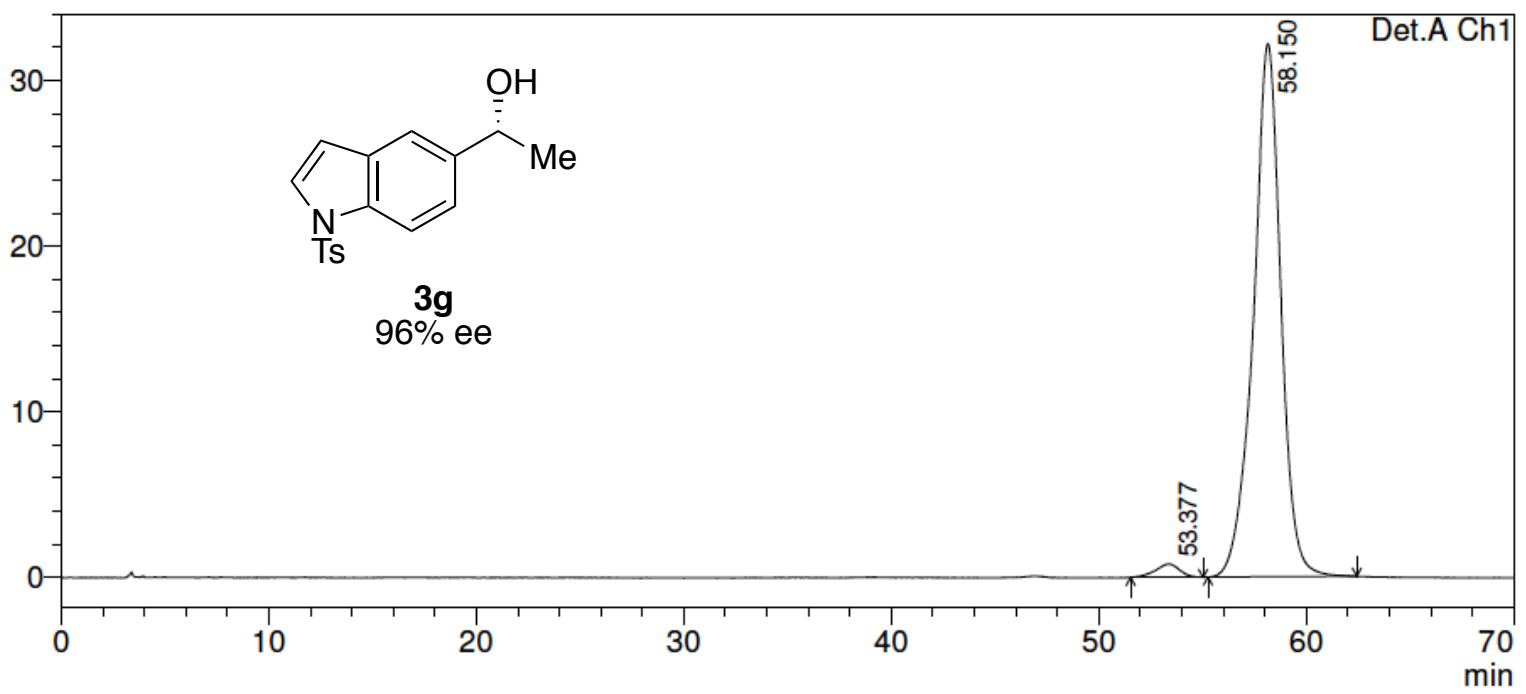

\begin{tabular}{|r|r|r|r|r|r|}
\hline \multicolumn{1}{|c|}{ Peak\# } & Ret. Time & \multicolumn{1}{c|}{ Area } & \multicolumn{1}{c|}{ Height } & Area \% & Height \% \\
\hline 1 & 53.377 & 64936 & 808 & 2.149 & 2.450 \\
\hline 2 & 58.150 & 2956179 & 32179 & 97.851 & 97.550 \\
\hline Total & & 3021114 & 32987 & 100.000 & 100.000 \\
\hline
\end{tabular}


Compound $3 \mathrm{~h}$, racemic ( $254 \mathrm{~nm}$ )

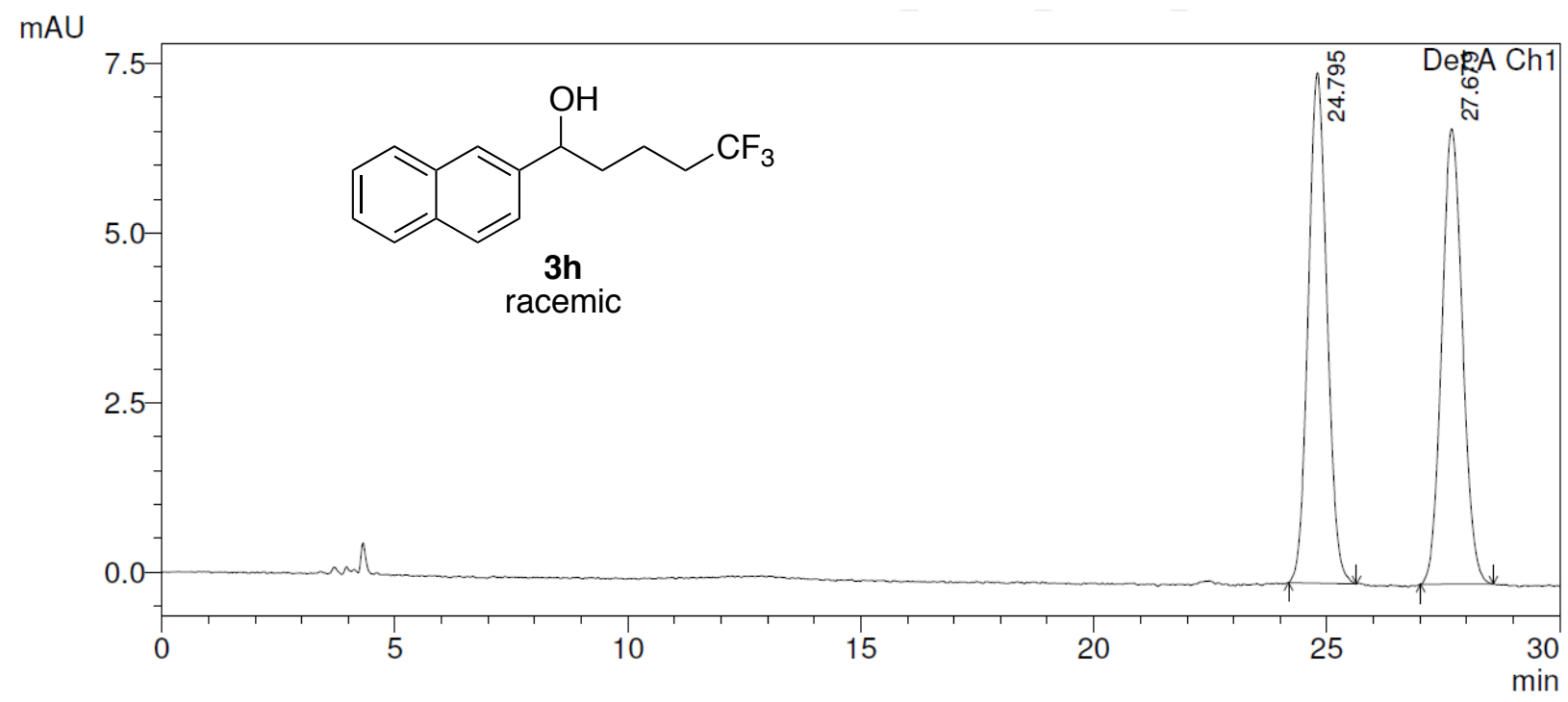

\begin{tabular}{|r|r|r|r|r|r|}
\hline \multicolumn{1}{|c|}{ Peak\# } & Ret. Time & \multicolumn{1}{c|}{ Area } & Height & Area \% & \multicolumn{1}{c|}{ Height \% } \\
\hline 1 & 24.795 & 210483 & 7521 & 50.324 & 52.855 \\
\hline 2 & 27.679 & 207772 & 6708 & 49.676 & 47.145 \\
\hline Total & & 418255 & 14229 & 100.000 & 100.000 \\
\hline
\end{tabular}

Compound $3 \mathrm{~h}, 96 \%$ ee ( $254 \mathrm{~nm}$ )

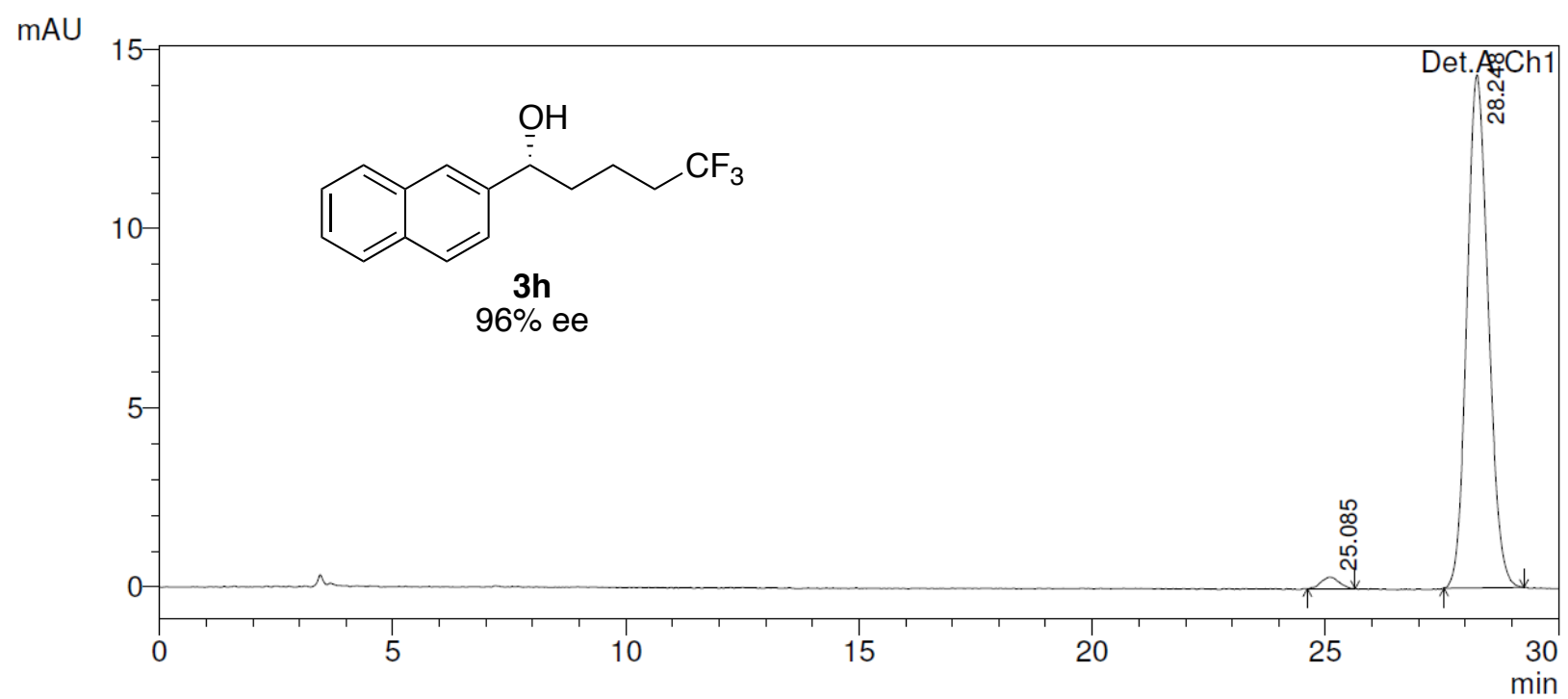

\begin{tabular}{|r|r|r|r|r|r|}
\hline \multicolumn{1}{|c|}{ Peak\# } & Ret. Time & \multicolumn{1}{c|}{ Area } & \multicolumn{1}{c|}{ Height } & Area \% & Height \% \\
\hline 1 & 25.085 & 8553 & 324 & 1.840 & 2.212 \\
\hline 2 & 28.248 & 456259 & 14308 & 98.160 & 97.788 \\
\hline Total & & 464812 & 14632 & 100.000 & 100.000 \\
\hline
\end{tabular}


Compound 3i, racemic (254 nm)

mAU

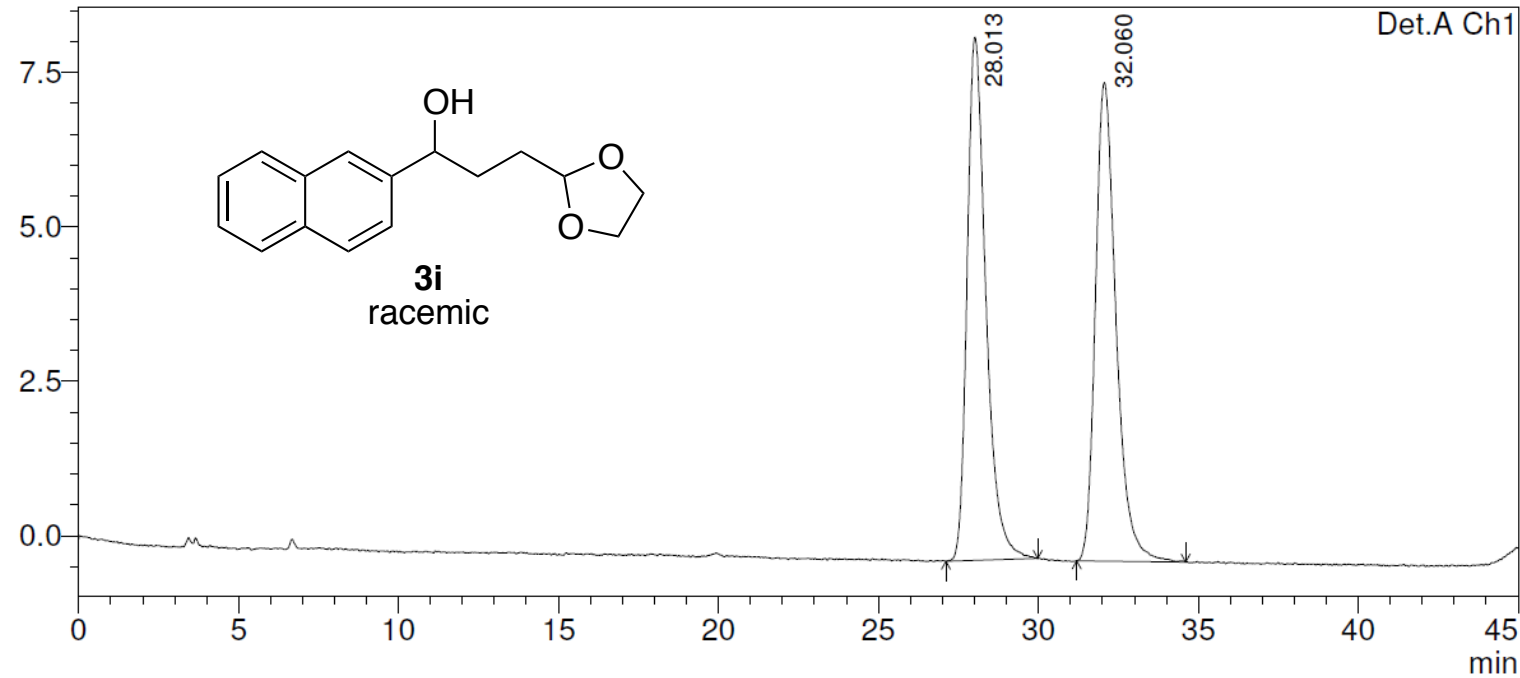

\begin{tabular}{|r|r|r|r|r|r|}
\hline \multicolumn{1}{|c|}{ Peak\# } & Ret. Time & \multicolumn{1}{c|}{ Area } & Height & Area \% & Height \% \\
\hline 1 & 28.013 & 346583 & 8466 & 49.977 & 52.224 \\
\hline 2 & 32.060 & 346898 & 7745 & 50.023 & 47.776 \\
\hline Total & & 693481 & 16211 & 100.000 & 100.000 \\
\hline
\end{tabular}

Compound $\mathbf{3 i}$, $98 \%$ ee $(254 \mathrm{~nm})$

mAU

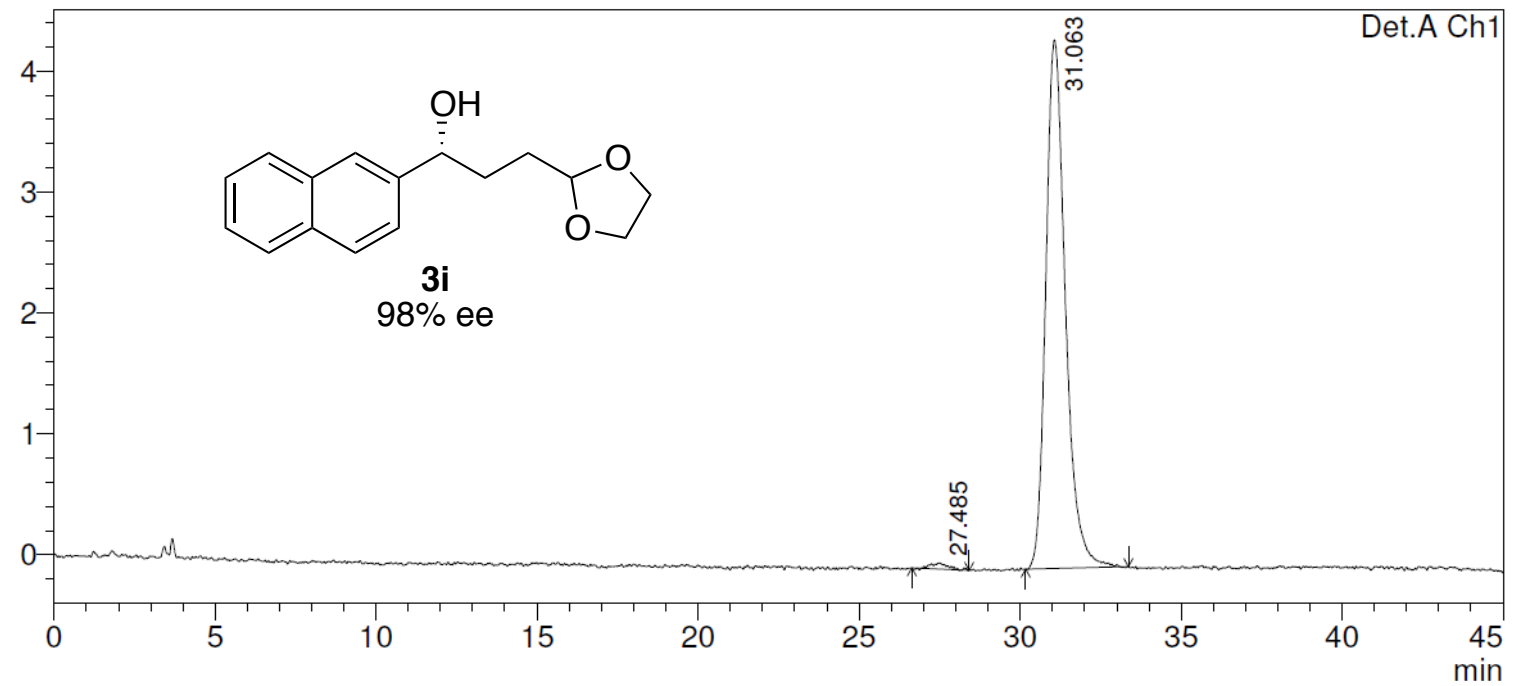

\begin{tabular}{|r|r|r|r|r|r|}
\hline \multicolumn{1}{|c|}{ Peak\# } & Ret. Time & \multicolumn{1}{c|}{ Area } & \multicolumn{1}{|c|}{ Height } & \multicolumn{1}{c|}{ Area \% } & Height $\%$ \\
\hline 1 & 27.485 & 1806 & 53 & 0.968 & 1.191 \\
\hline 2 & 31.063 & 184751 & 4374 & 99.032 & 98.809 \\
\hline Total & & 186557 & 4426 & 100.000 & 100.000 \\
\hline
\end{tabular}


Compound 3j, racemic (254 nm)

mAU

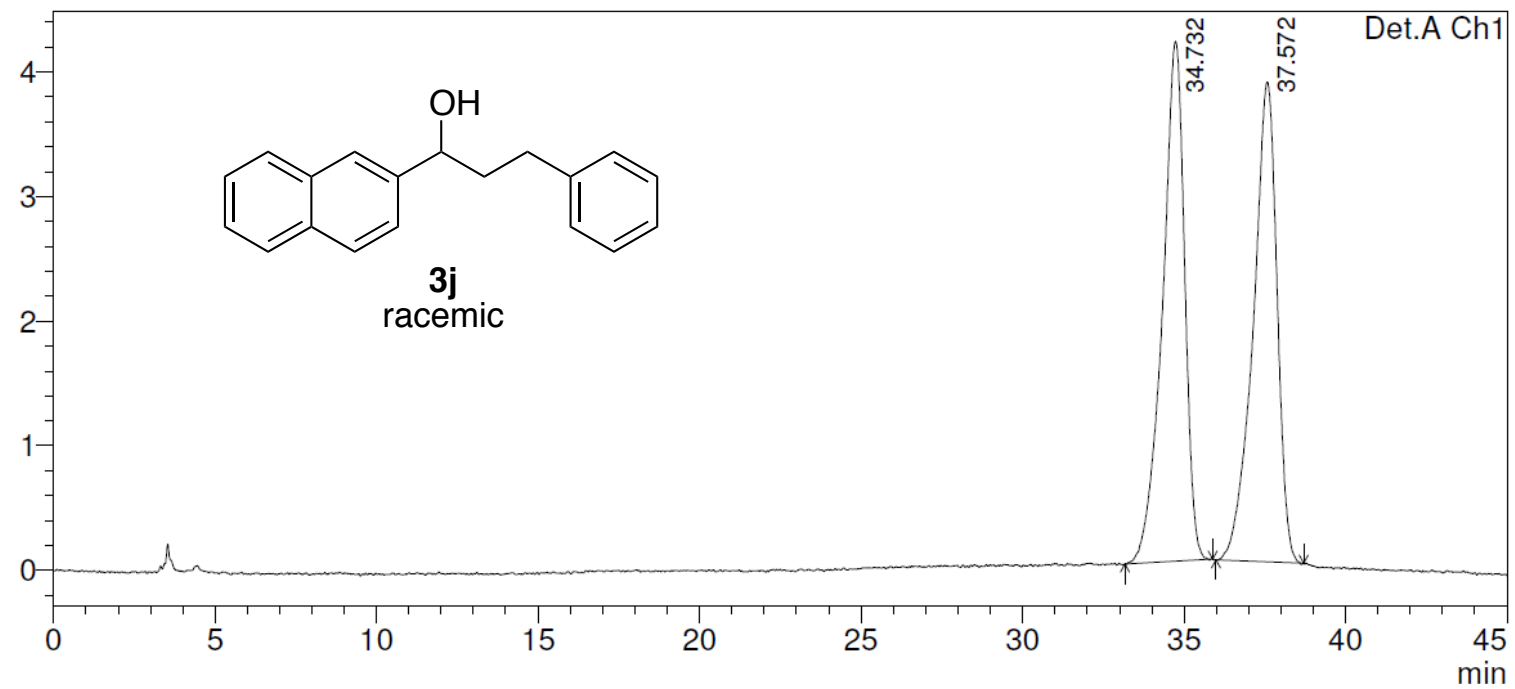

\begin{tabular}{|r|r|r|r|r|r|}
\hline \multicolumn{1}{|c|}{ Peak\# } & Ret. Time & \multicolumn{1}{c|}{ Area } & Height & Area \% & Height \% \\
\hline 1 & 34.732 & 198596 & 4170 & 50.141 & 51.978 \\
\hline 2 & 37.572 & 197482 & 3853 & 49.859 & 48.022 \\
\hline Total & & 396078 & 8023 & 100.000 & 100.000 \\
\hline
\end{tabular}

Compound $\mathbf{3 j}$, $98 \%$ ee $(254 \mathrm{~nm})$

mAU

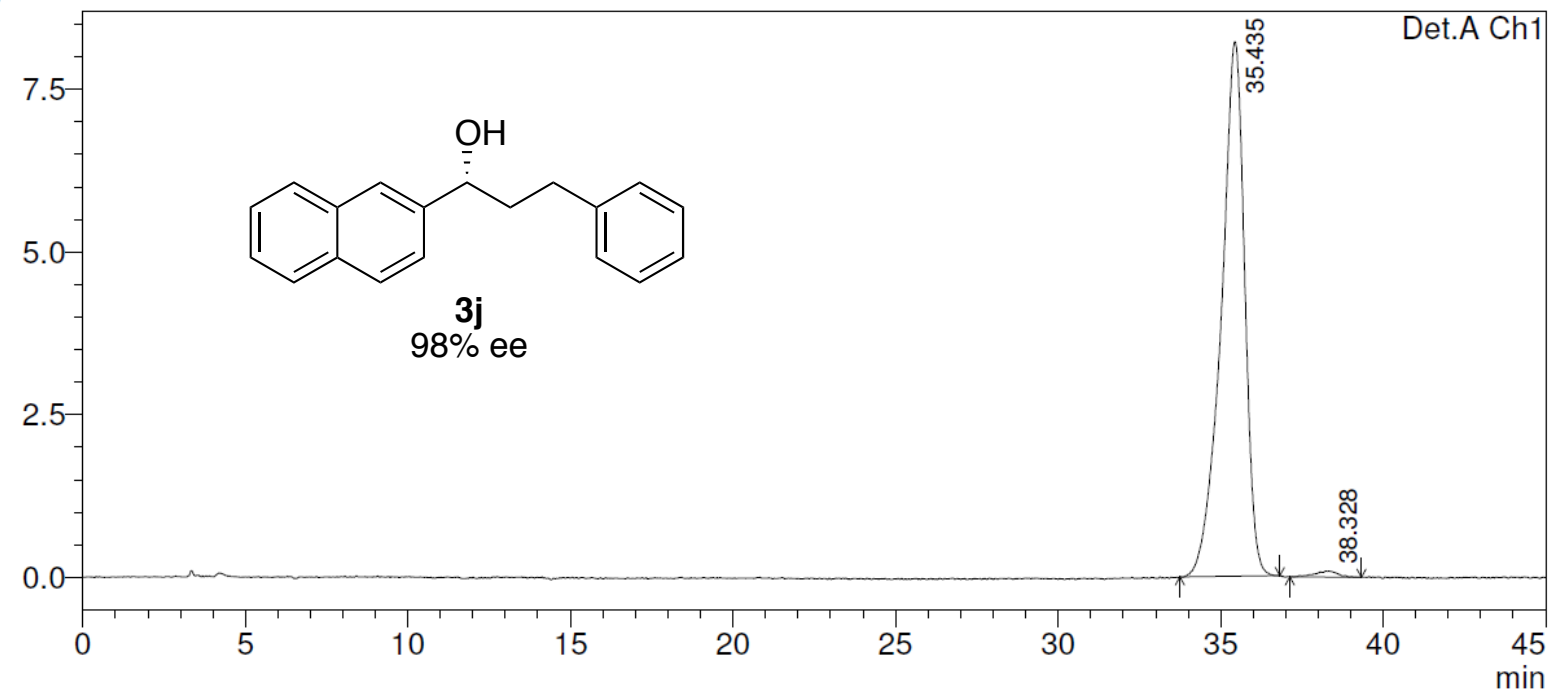

\begin{tabular}{|r|r|r|r|r|r|}
\hline \multicolumn{1}{|c|}{ Peak\# } & Ret. Time & \multicolumn{1}{c|}{ Area } & \multicolumn{1}{|c|}{ Height } & Area \% & Height \% \\
\hline 1 & 35.435 & 412336 & 8222 & 98.853 & 98.861 \\
\hline 2 & 38.328 & 4783 & 95 & 1.147 & 1.139 \\
\hline Total & & 417119 & 8317 & 100.000 & 100.000 \\
\hline
\end{tabular}


Compound 3k, racemic (254 nm)

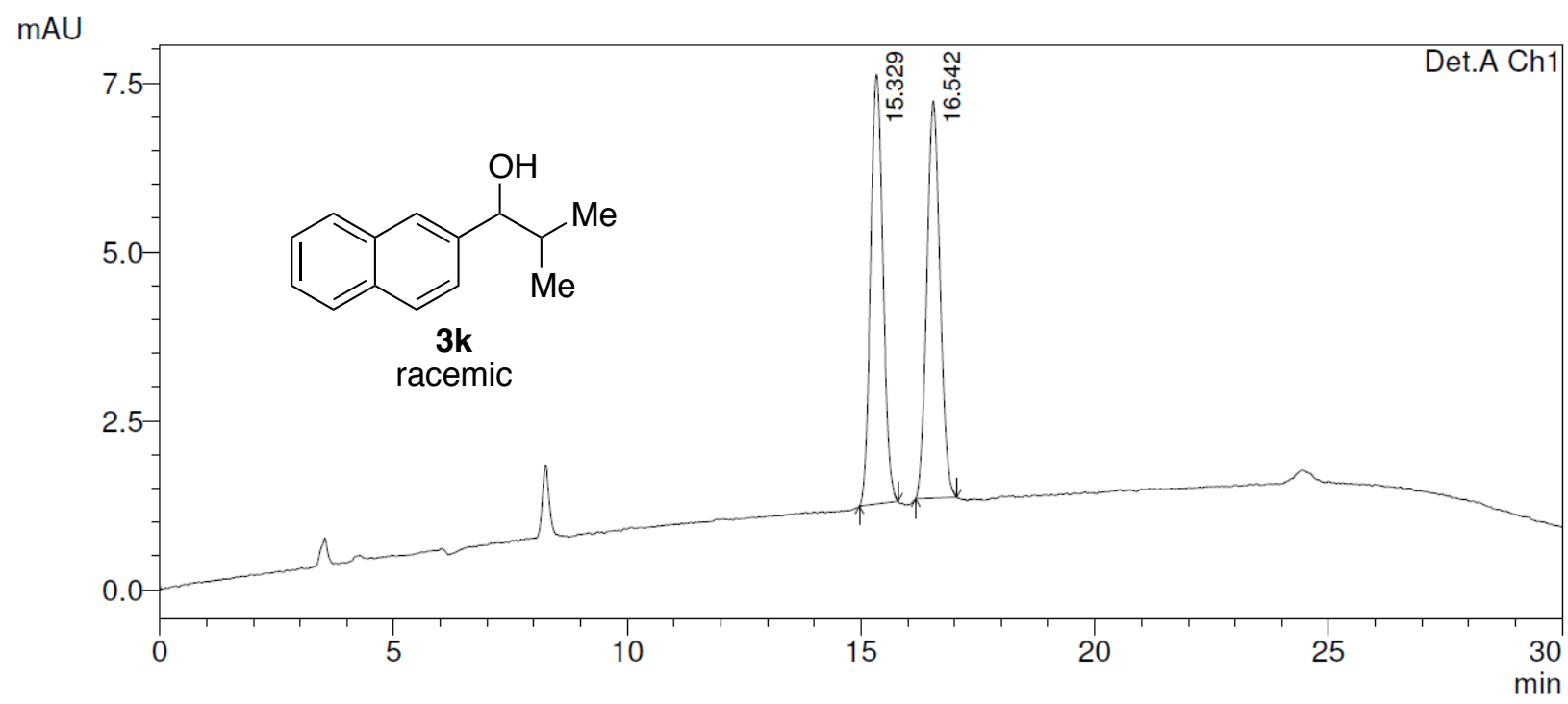

\begin{tabular}{|r|r|r|r|r|r|}
\hline \multicolumn{1}{|c|}{ Peak\# } & Ret. Time & \multicolumn{1}{c|}{ Area } & Height & Area \% & \multicolumn{1}{c|}{ Height \% } \\
\hline 1 & 15.329 & 116684 & 6363 & 50.105 & 51.956 \\
\hline 2 & 16.542 & 116193 & 5884 & 49.895 & 48.044 \\
\hline Total & & 232877 & 12246 & 100.000 & 100.000 \\
\hline
\end{tabular}

Compound 3k, 98\% ee (254 nm)

mAU

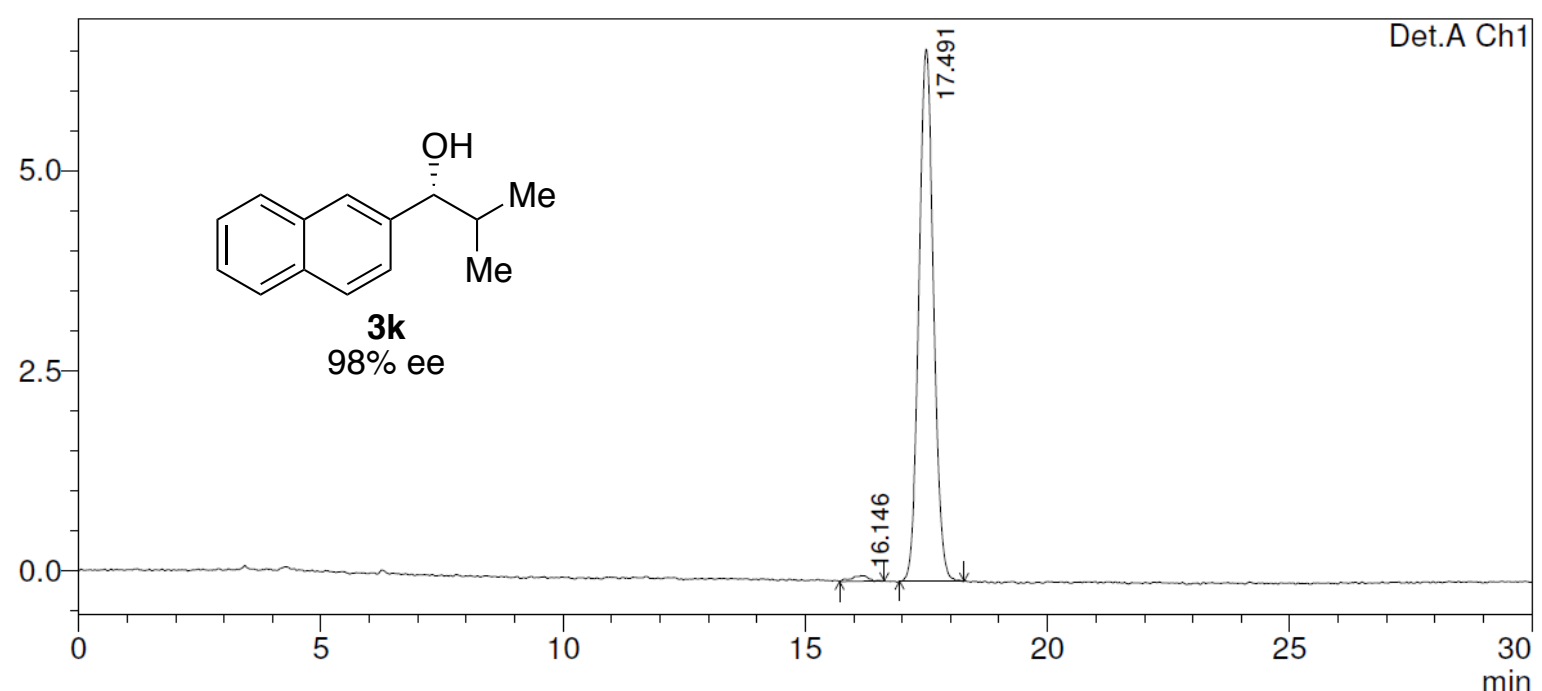

\begin{tabular}{|r|r|r|r|r|r|}
\hline \multicolumn{1}{|c|}{ Peak\# } & Ret. Time & \multicolumn{1}{|c|}{ Area } & \multicolumn{1}{|c|}{ Height } & Area \% & Height \% \\
\hline 1 & 16.146 & 1594 & 70 & 1.126 & 1.034 \\
\hline 2 & 17.491 & 140016 & 6657 & 98.874 & 98.966 \\
\hline Total & & 141610 & 6726 & 100.000 & 100.000 \\
\hline
\end{tabular}


Compound 3a, racemic (254 nm)

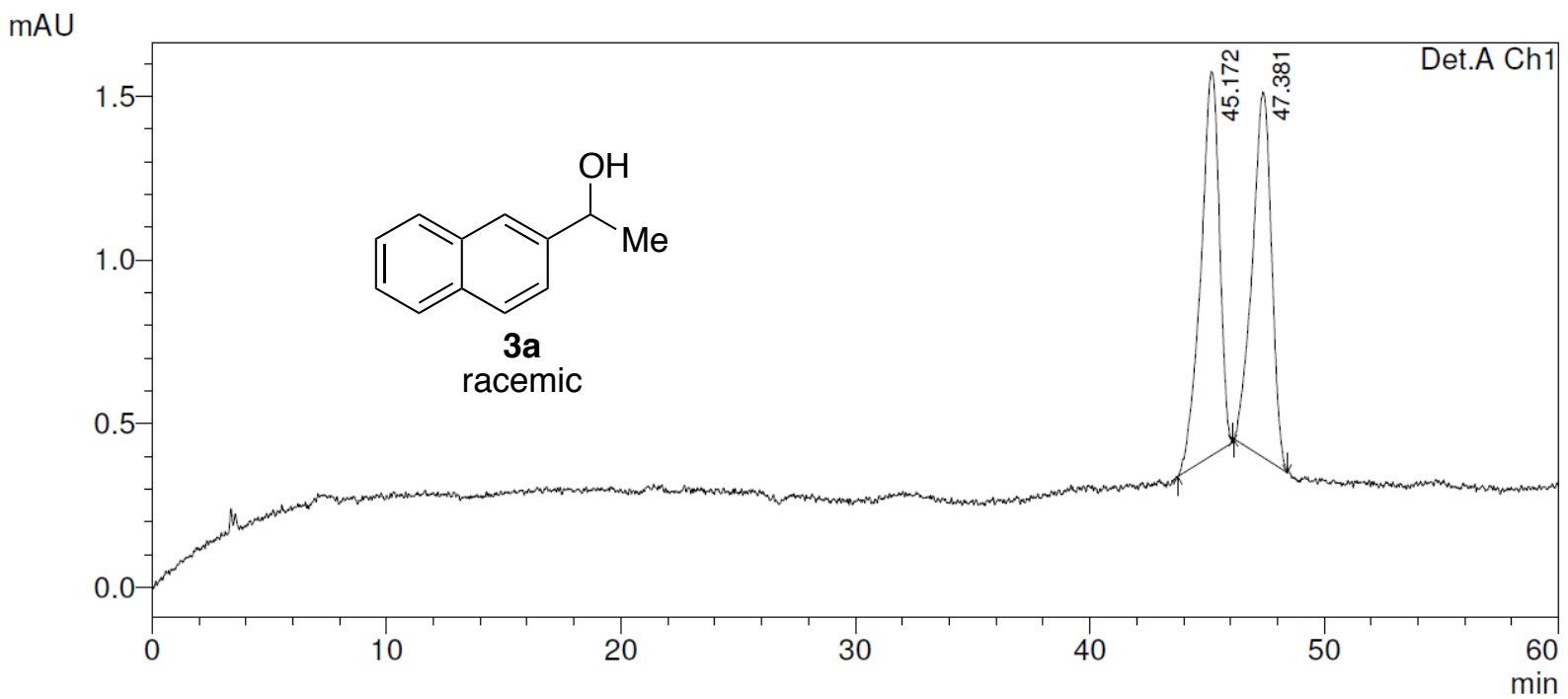

\begin{tabular}{|r|r|r|r|r|r|}
\hline \multicolumn{1}{|c|}{ Peak\# } & Ret. Time & \multicolumn{1}{c|}{ Area } & \multicolumn{1}{|c|}{ Height } & Area \% & Height \% \\
\hline 1 & 45.172 & 64627 & 1174 & 50.417 & 51.245 \\
\hline 2 & 47.381 & 63558 & 1117 & 49.583 & 48.755 \\
\hline Total & & 128185 & 2292 & 100.000 & 100.000 \\
\hline
\end{tabular}

Compound 3a, 95\% ee (254 nm)

mAU

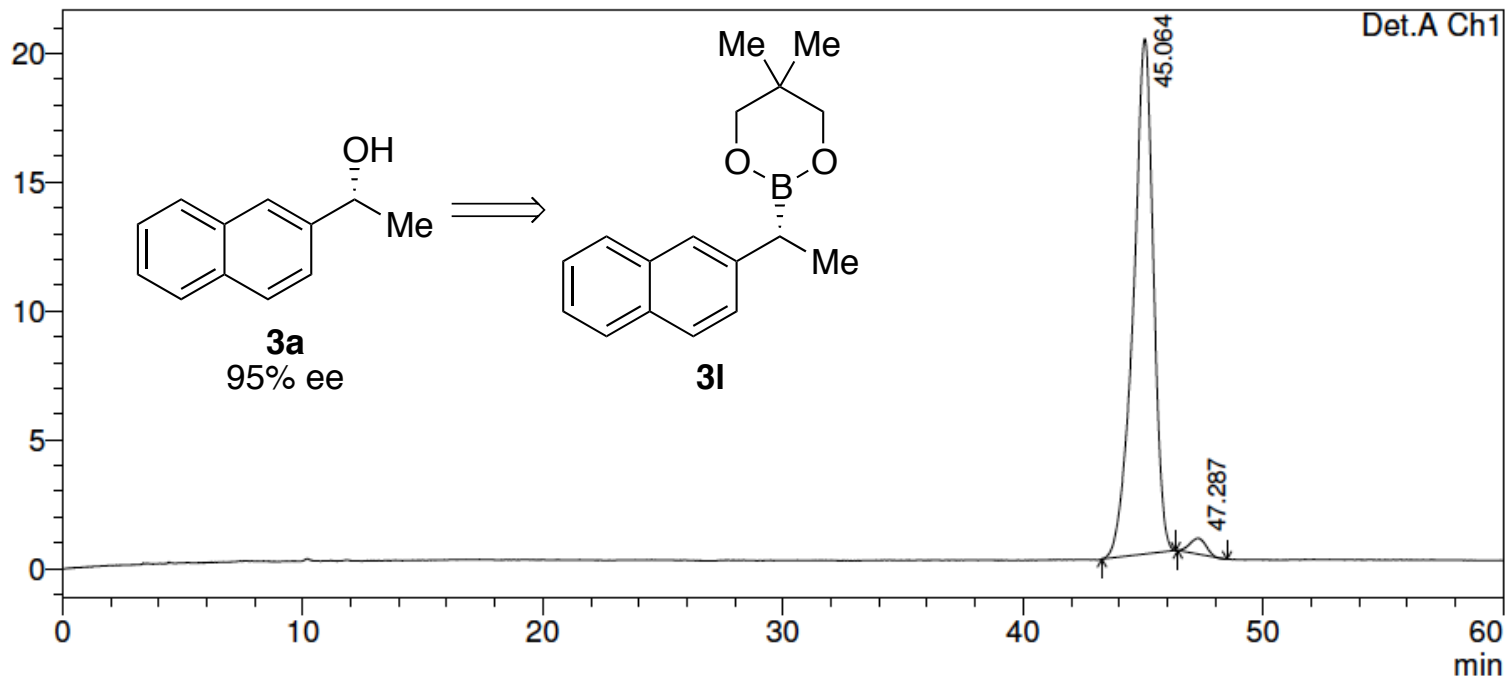

\begin{tabular}{|r|r|r|r|r|r|}
\hline \multicolumn{1}{|c|}{ Peak\# } & \multicolumn{1}{|c|}{ Ret. Time } & \multicolumn{1}{c|}{ Area } & \multicolumn{1}{c|}{ Height } & \multicolumn{1}{c|}{ Area \% } & Height \% \\
\hline 1 & 45.064 & 1159668 & 20005 & 97.571 & 97.019 \\
\hline 2 & 47.287 & 28864 & 615 & 2.429 & 2.981 \\
\hline Total & & 1188532 & 20620 & 100.000 & 100.000 \\
\hline
\end{tabular}


Compound 3a, racemic (254 nm)

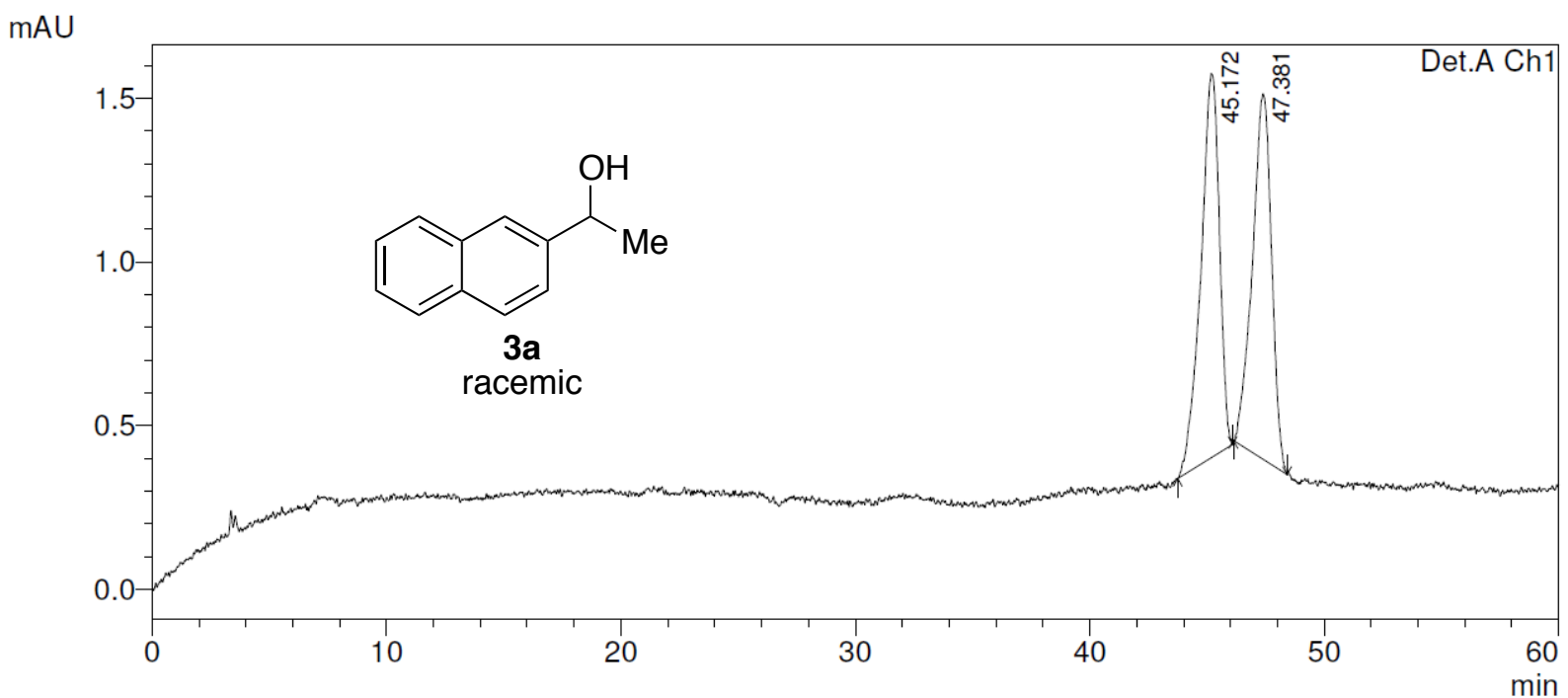

\begin{tabular}{|r|r|r|r|r|r|}
\hline \multicolumn{1}{|c|}{ Peak\# } & Ret. Time & \multicolumn{1}{c|}{ Area } & \multicolumn{1}{|c|}{ Height } & Area \% & Height \% \\
\hline 1 & 45.172 & 64627 & 1174 & 50.417 & 51.245 \\
\hline 2 & 47.381 & 63558 & 1117 & 49.583 & 48.755 \\
\hline Total & & 128185 & 2292 & 100.000 & 100.000 \\
\hline
\end{tabular}

Compound 3a, 95\% ee (254 nm)

mAU

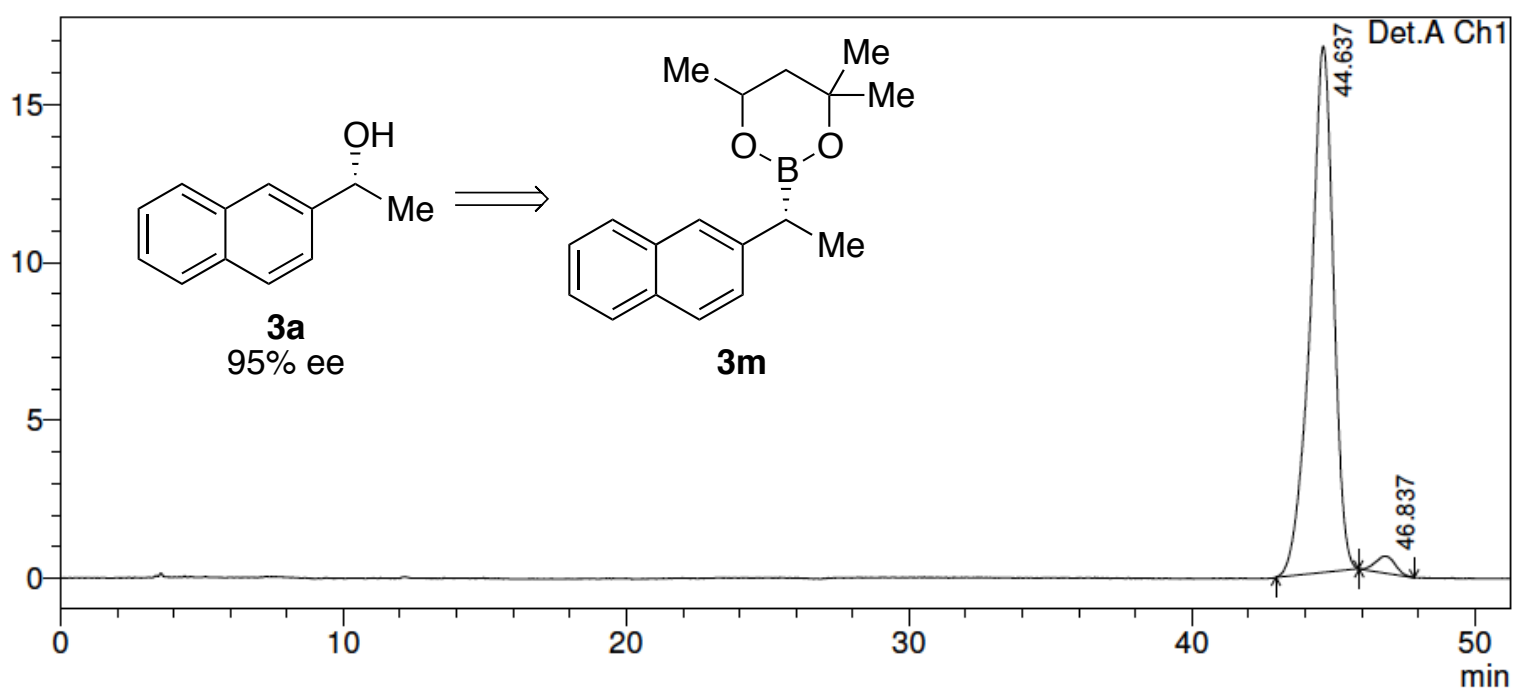

\begin{tabular}{|r|r|r|r|r|r|}
\hline \multicolumn{1}{|c|}{ Peak\# } & \multicolumn{1}{|c|}{ Ret. Time } & \multicolumn{1}{c|}{ Area } & \multicolumn{1}{c|}{ Height } & \multicolumn{1}{c|}{ Area \% } & \multicolumn{1}{c|}{ Height \% } \\
\hline 1 & 44.637 & 946536 & 16673 & 97.303 & 96.868 \\
\hline 2 & 46.837 & 26234 & 539 & 2.697 & 3.132 \\
\hline Total & & 972770 & 17212 & 100.000 & 100.000 \\
\hline
\end{tabular}


Compound $3 n$, racemic ( $254 \mathrm{~nm}$ )

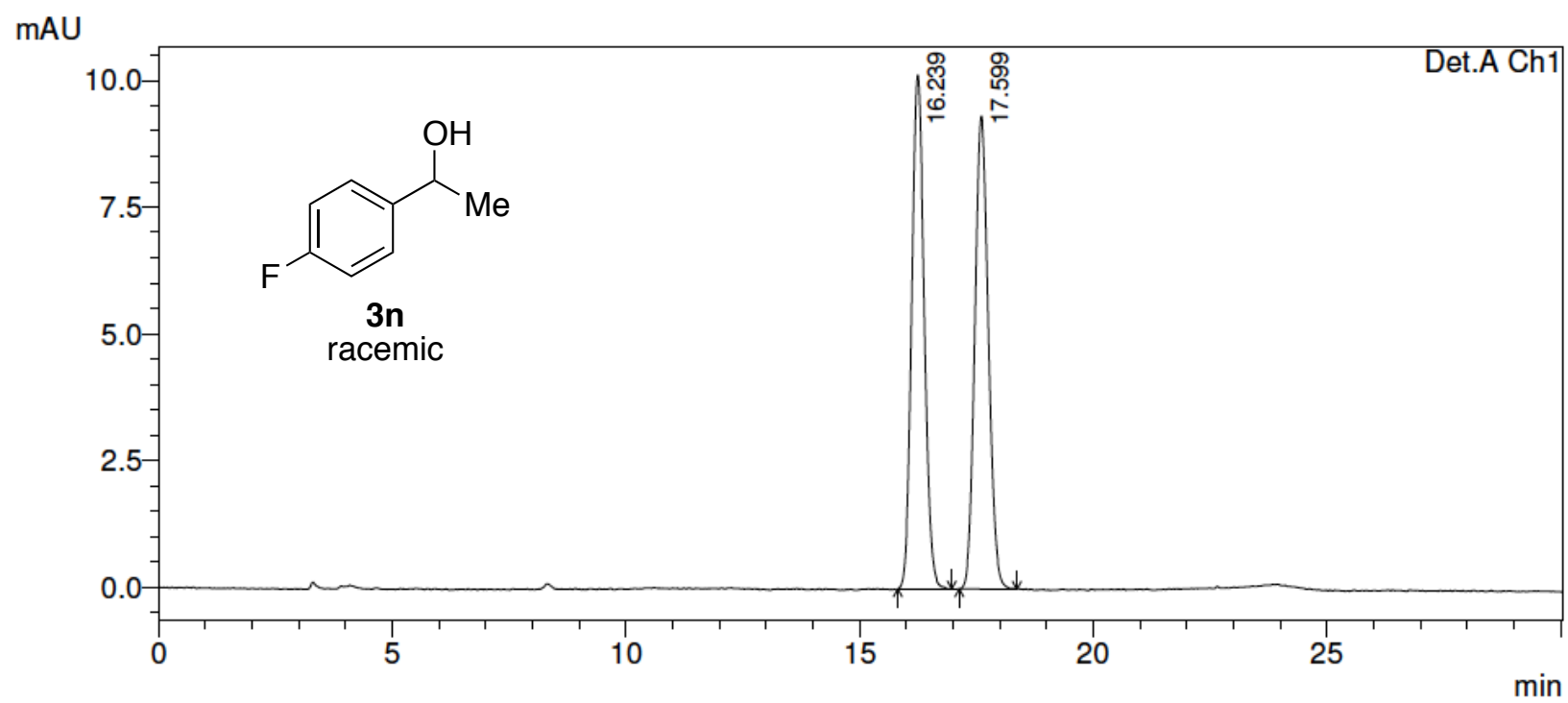

\begin{tabular}{|r|r|r|r|r|r|}
\hline \multicolumn{1}{|c|}{ Peak\# } & Ret. Time & \multicolumn{1}{c|}{ Area } & Height & Area \% & Height \% \\
\hline 1 & 16.239 & 189291 & 10142 & 50.049 & 52.077 \\
\hline 2 & 17.599 & 188917 & 9333 & 49.951 & 47.923 \\
\hline Total & & 378208 & 19476 & 100.000 & 100.000 \\
\hline
\end{tabular}

Compound $3 n$, $85 \%$ ee $(254 \mathrm{~nm})$

mAU

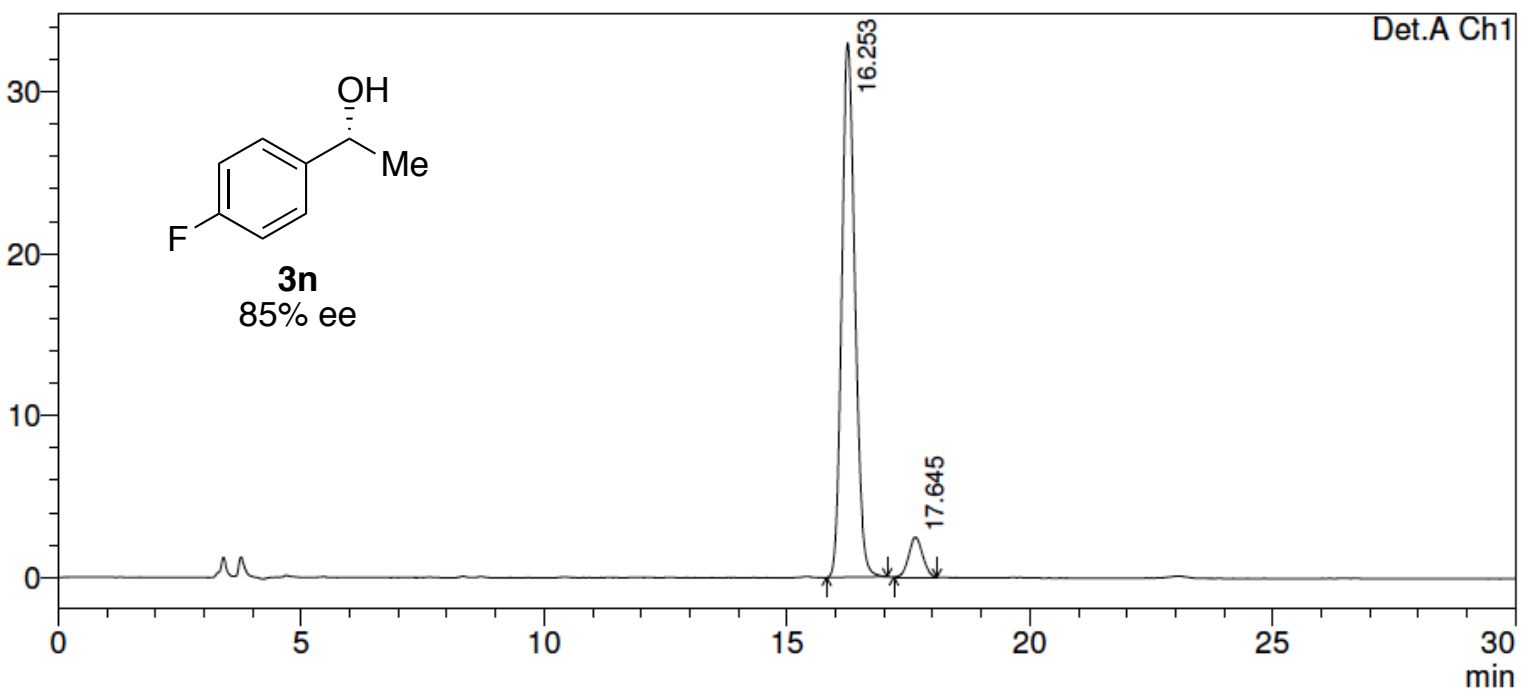

\begin{tabular}{|r|r|r|r|r|r|}
\hline \multicolumn{1}{|c|}{ Peak\# } & Ret. Time & \multicolumn{1}{c|}{ Area } & Height & Area \% & Height \% \\
\hline 1 & 16.253 & 625785 & 33025 & 92.796 & 93.094 \\
\hline 2 & 17.645 & 48585 & 2450 & 7.204 & 6.906 \\
\hline Total & & 674370 & 35475 & 100.000 & 100.000 \\
\hline
\end{tabular}


Compound 3o, racemic (254 nm)

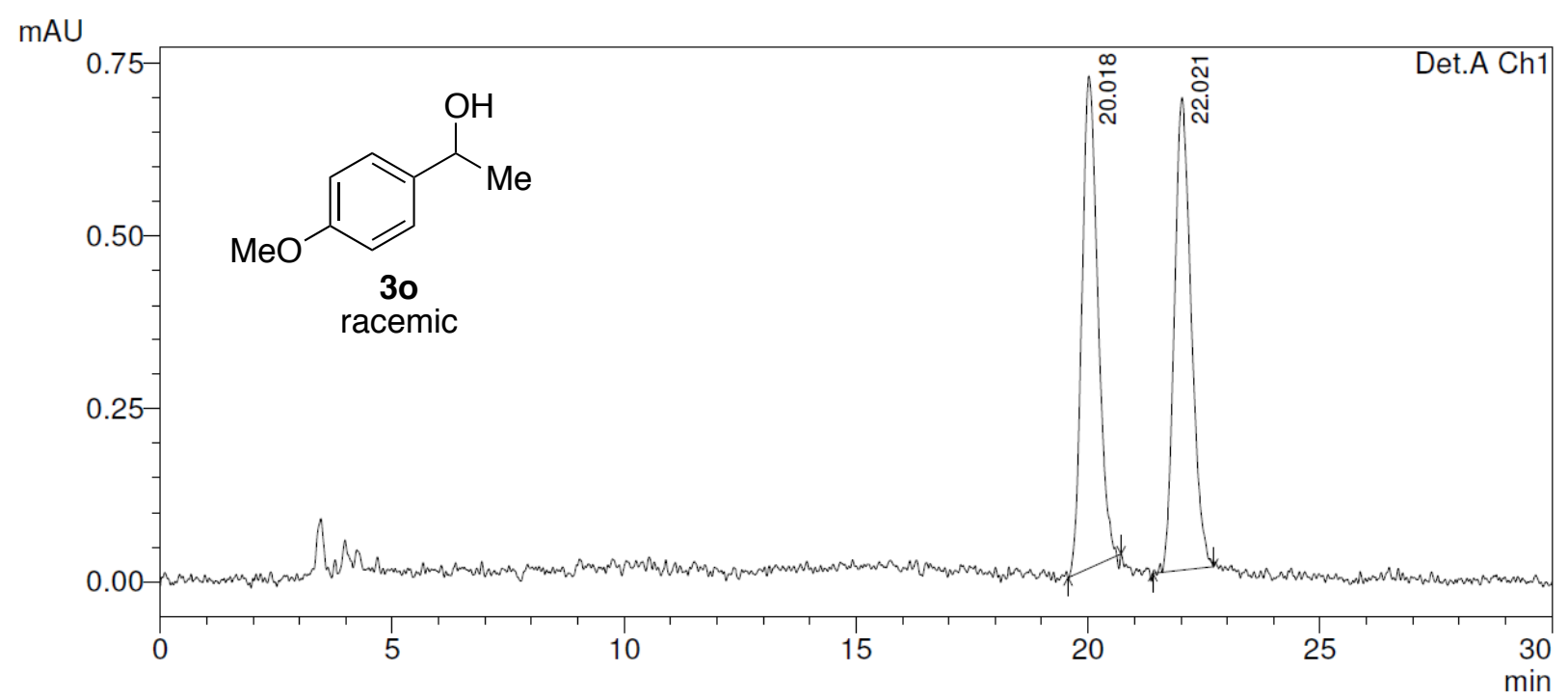

\begin{tabular}{|r|r|r|r|r|r|}
\hline \multicolumn{1}{|c|}{ Peak\# } & Ret. Time & \multicolumn{1}{c|}{ Area } & \multicolumn{1}{|c|}{ Height } & Area \% & Height \% \\
\hline 1 & 20.018 & 16955 & 713 & 49.424 & 51.024 \\
\hline 2 & 22.021 & 17351 & 684 & 50.576 & 48.976 \\
\hline Total & & 34306 & 1397 & 100.000 & 100.000 \\
\hline
\end{tabular}

Compound 3o, 87\% ee (254 nm)

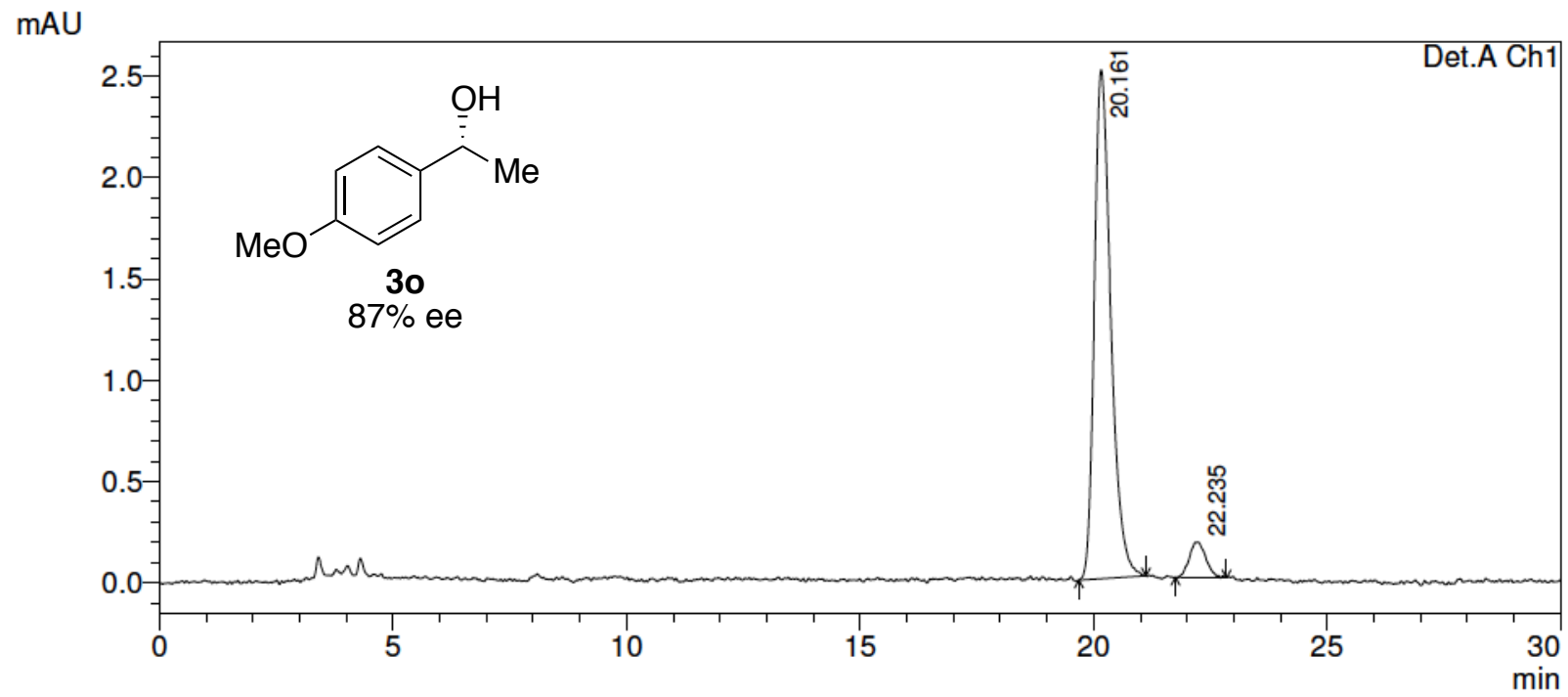

\begin{tabular}{|r|r|r|r|r|r|}
\hline \multicolumn{1}{|c|}{ Peak\# } & Ret. Time & \multicolumn{1}{c|}{ Area } & \multicolumn{1}{c|}{ Height } & Area \% & Height \% \\
\hline 1 & 20.161 & 61611 & 2512 & 93.425 & 93.488 \\
\hline 2 & 22.235 & 4336 & 175 & 6.575 & 6.512 \\
\hline Total & & 65947 & 2687 & 100.000 & 100.000 \\
\hline
\end{tabular}


Compound $3 p$, racemic $(210 \mathrm{~nm})$

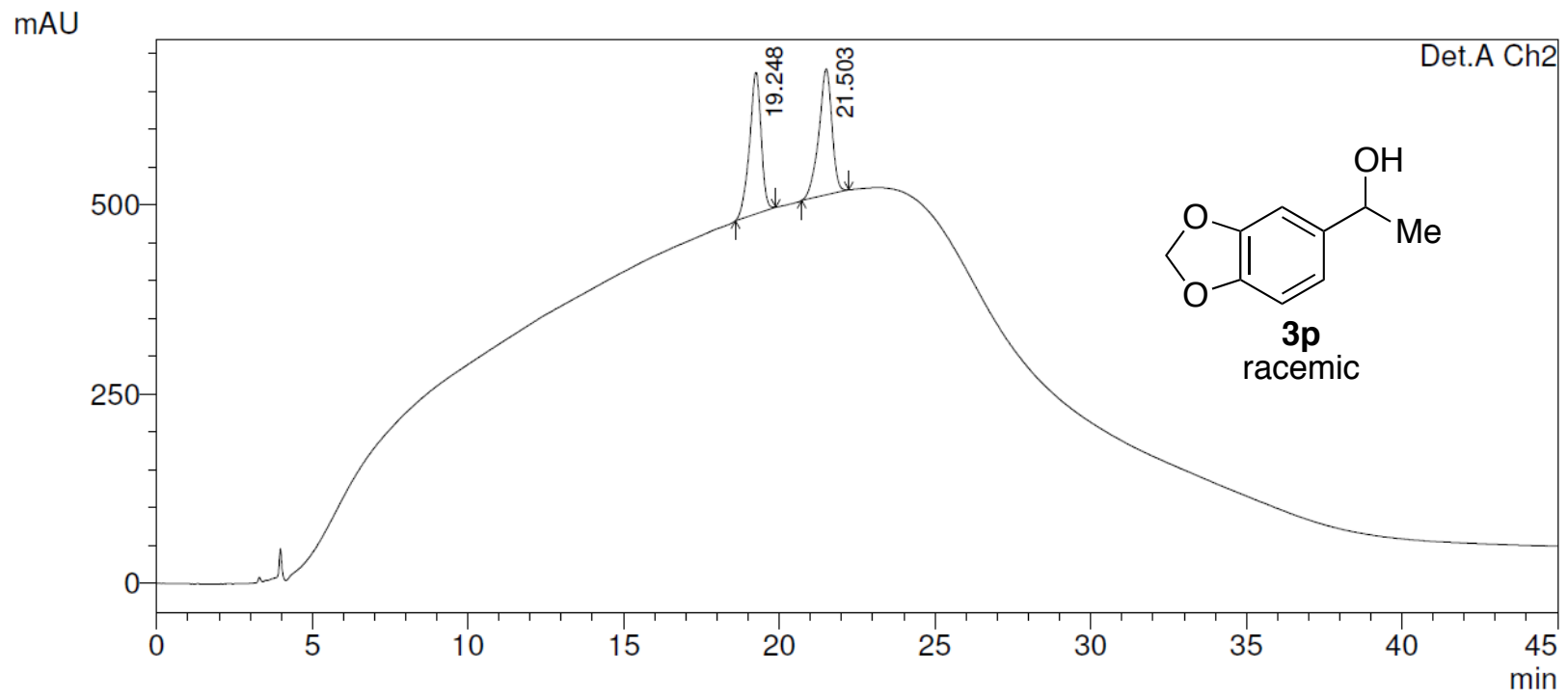

\begin{tabular}{|r|r|r|r|r|r|}
\hline \multicolumn{1}{|c|}{ Peak\# } & Ret. Time & Area & Height & Area \% & Height \% \\
\hline 1 & 19.248 & 4853386 & 187177 & 49.816 & 52.913 \\
\hline 2 & 21.503 & 4889175 & 166570 & 50.184 & 47.087 \\
\hline Total & & 9742560 & 353746 & 100.000 & 100.000 \\
\hline
\end{tabular}

Compound $3 p$, $85 \%$ ee $(210 \mathrm{~nm})$

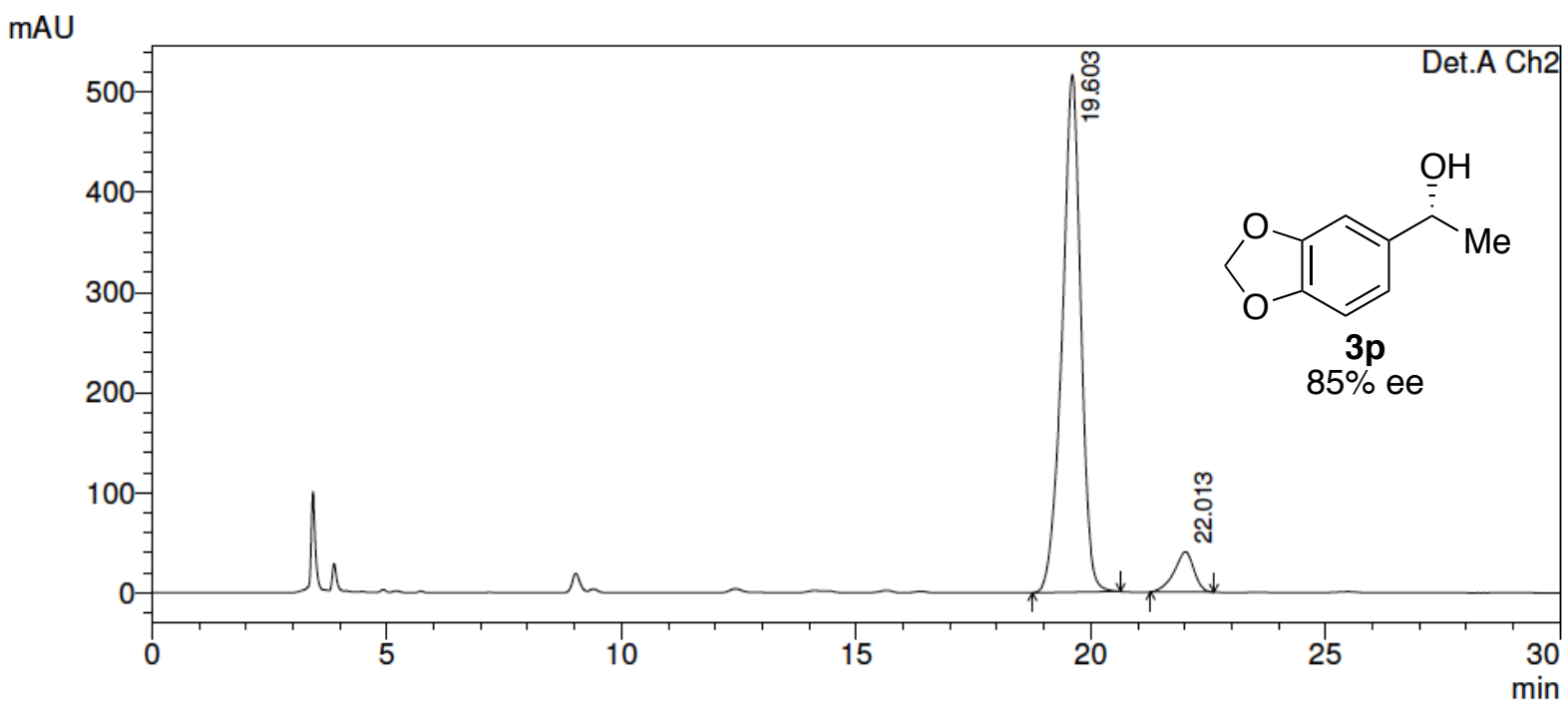

\begin{tabular}{|r|r|r|r|r|r|}
\hline Peak\# & Ret. Time & \multicolumn{1}{c|}{ Area } & \multicolumn{1}{c|}{ Height } & Area \% & Height \% \\
\hline 1 & 19.603 & 14324275 & 516565 & 92.465 & 92.804 \\
\hline 2 & 22.013 & 1167262 & 40056 & 7.535 & 7.196 \\
\hline Total & & 15491537 & 556621 & 100.000 & 100.000 \\
\hline
\end{tabular}


Compound 3c, racemic (254 nm)

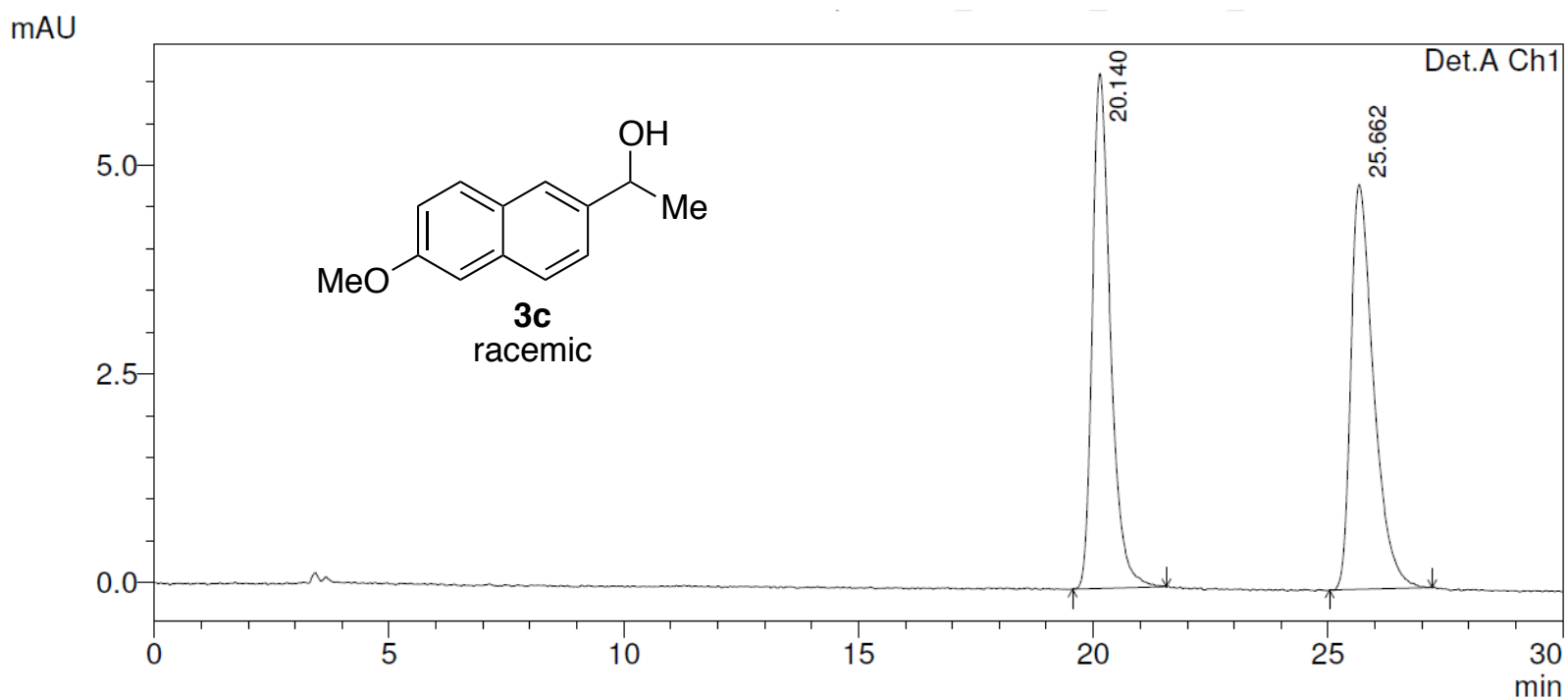

\begin{tabular}{|r|r|r|r|r|r|}
\hline \multicolumn{1}{|c|}{ Peak\# } & Ret. Time & \multicolumn{1}{c|}{ Area } & Height & Area \% & Height \% \\
\hline 1 & 20.140 & 165137 & 6165 & 50.153 & 55.982 \\
\hline 2 & 25.662 & 164128 & 4848 & 49.847 & 44.018 \\
\hline Total & & 329266 & 11013 & 100.000 & 100.000 \\
\hline
\end{tabular}

Compound 3c, 99\% ee (254 nm)

mAU

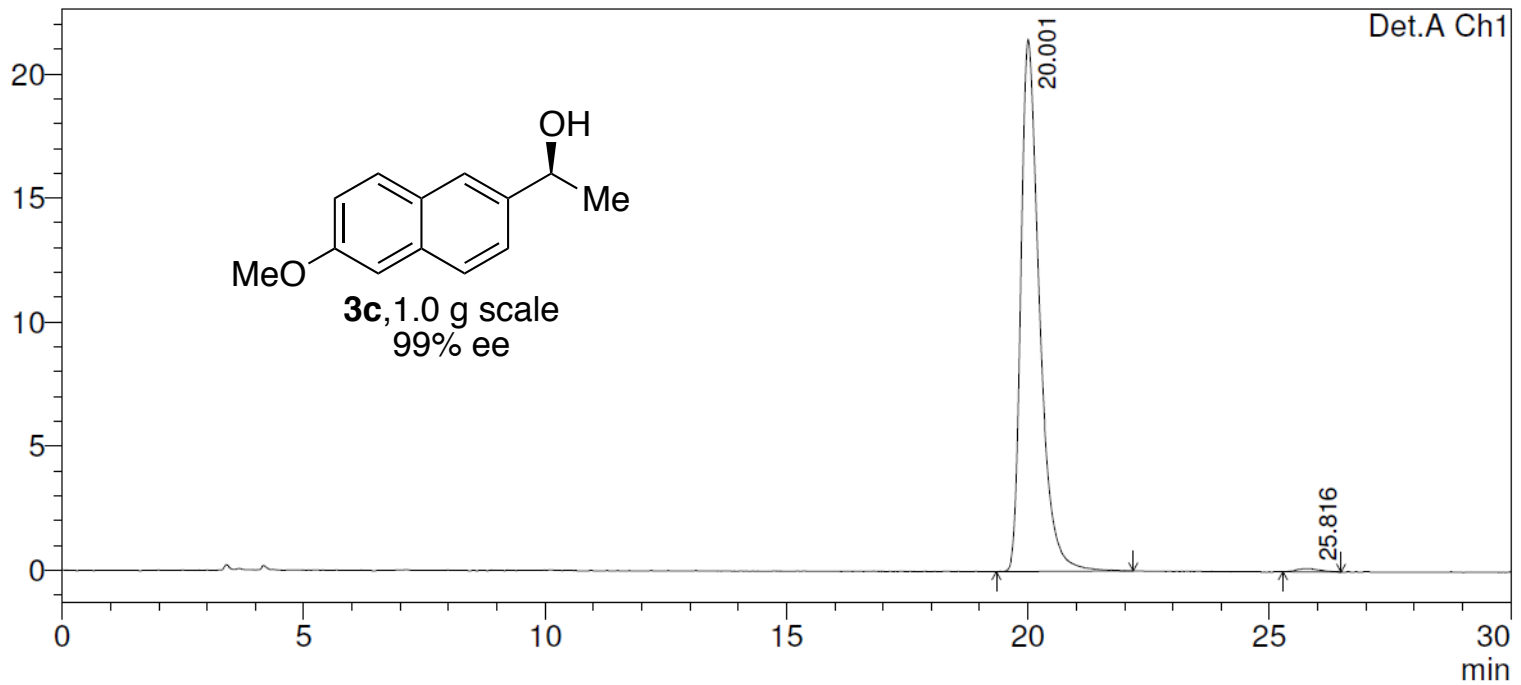

\begin{tabular}{|r|r|r|r|r|r|}
\hline Peak\# & Ret. Time & \multicolumn{1}{c|}{ Area } & Height & Area \% & \multicolumn{1}{c|}{ Height \% } \\
\hline 1 & 20.001 & 574869 & 21457 & 99.271 & 99.399 \\
\hline 2 & 25.816 & 4222 & 130 & 0.729 & 0.601 \\
\hline Total & & 579091 & 21587 & 100.000 & 100.000 \\
\hline
\end{tabular}

